HNF-3602

Volume 1 of 2

\title{
Volume 1: Calculating \\ Potential to Emit Releases and Doses for FEMPs and NOCs
}

Prepared for the U.S. Department of Energy Assistant Secretary for Environmental Management

FWOR DANMEL HNHFOR, ANC.

Richland, Washington

RECORD COPY

Hanford Management and Integration Contractor for the

U.S. Department of Energy under Contract DE-AC06-96RL13200

Approved for Public Release; Further Dissemination Unlimited 


\section{Volume 1: Calculating Potential to Emit Releases and Doses for FEMPs and NOCs}

Prepared by:

J. S. Hill

Waste Management Federal Services of Hanford, Inc.

P. D. Rittmann

Fluor Daniel Northwest

Date Published

July 1999

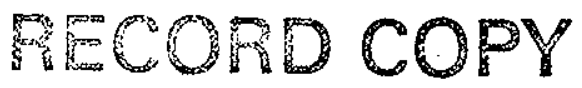

Prepared for the U.S. Department of Energy

Assistant Secretary for Environmental Management



Richland, Washington

Hanford Management and Integration Contractor for the

U.S. Department of Energy under Contract DE-ACO6-96RL13200

Approved for Public Release; Further Dissemination Unlimited 


\section{RELEASE AUTHORIZATION}

\section{Document}

Number:

Document

Title:
HNF-3602, Vol. 1, Rev. 0

Volume 1: Calculating Potential to Emit Releases

and Doses for FEMPs and NOCs

\section{This document, reviewed in accordance with DOE Order 241.1, "Scientific and Technical Information Management," and DOE G 241.1-1, "Guide to the Management of Scientific and Technical Information," does not contain classified or sensitive unclassified information and is:}

\section{APPROVED FOR PUBLIC RELEASE}

\section{Chistires Sritlingtean \\ C. Wolingham \\ $\therefore \quad$ Lockheed Martin Services, Inc. \\ Document Control/Information Clearance}

$7 / 27 / 99$

Reviewed for Applied Technology, Business Sensitive, Classified, Copyrighted, Export Controlled, Patent Personal/Private, Proprietary, Protected CRADA, Trademark, Unclassified Controlled Nuclear Information.

LEGAL DISCLAIMER. This report was prepared as an account of work sponsored by an agency of the United States Government. Neither the United States Government nor any agency thereof, not any of their employees, nor any of their contractors, subcontractors or their employees, makes any warranty, express or implied, or assumes any legal liability or responsibility for the accuracy, completeness, or any third party's use or the results of such use of any information, apparatus, product, or process disclosed, or represents that its use would not infringe privately owned rights. Reference herein to any specific commercial product, process, or service by trade name, trademark, manufacturer, or otherwise, does not necessarily constitute or imply its endorsement, recommendation, or favoring by the United states Government or any agency thereof or its contractors or subcontractors. The views and opinions of authors expressed herein do not necessarily state or reflect those of the United States Government or any agency thereof. This report has been reproduced from the best available copy. Printed in the United States of America. 
HNF-3602, Vol. 1

\section{APPROVAL PAGE}

Document Title: CALCULATING POTENTIAL TO EMIT RELEASES AND DOSES FOR FEMPS AND NOCs

Prepared by: $\frac{0.14010}{\text { S. Hill }} \frac{2 / 7 / 99}{\text { Date }}$

Nir \& Water Services

Waste Management Federal Services of Hanford, Inc.

Prepared by: $\frac{\text { Payf Nottiviann }}{\text { P. D. Rittmann }} \frac{7-12-99}{\text { Date }}$

Environmental \& Nuclear Initiatives

Fluor Daniel Northwest, Inc.

Approved by:

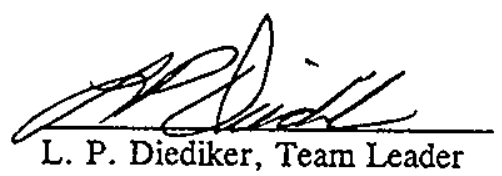

Air \& Water Services

$\frac{7-7-99}{\text { Date }}$

Waste Management Federal Services of Hanford, Inc.

Approved by:

EMin M. Greagr

E. M. Greager, Manager

Air \& Water Services

Waste Management Federal Services of Hanford, Inc.

Approved by:

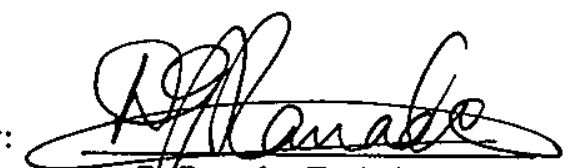

D. G Ranade, Technical Representative

Environmental Integration

Fluor Daniel Hanford, Inc.
$7 / 8 / 99$

Date

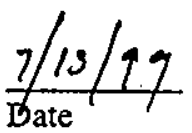




\section{CONTENTS}

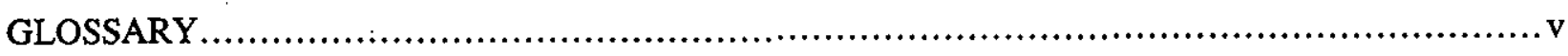



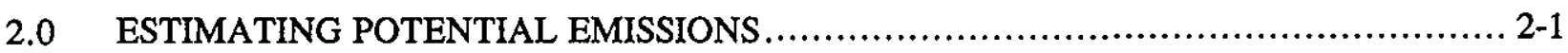

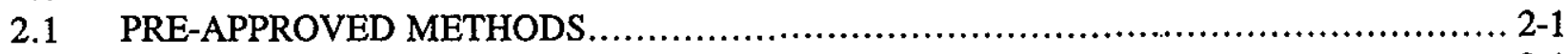

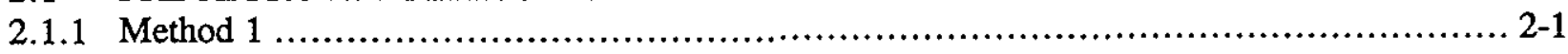

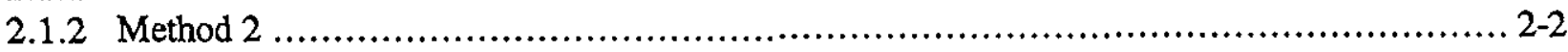

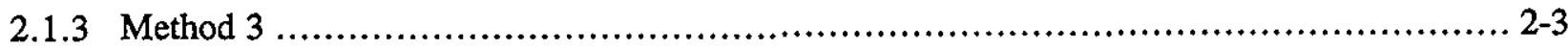



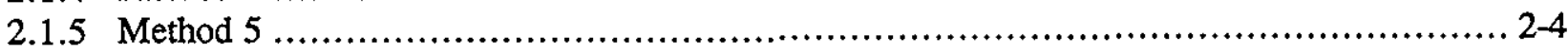

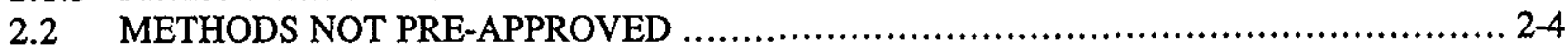

3.0 CALCULATING OFFSITE DOSES TO THE NEAREST RECEPTOR ..................... 3-1

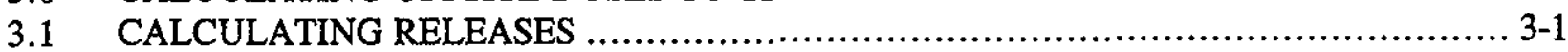

3.2 SELECTING THE APPROPRIATE UNIT DOSE FACTORS .............................. $3-2$

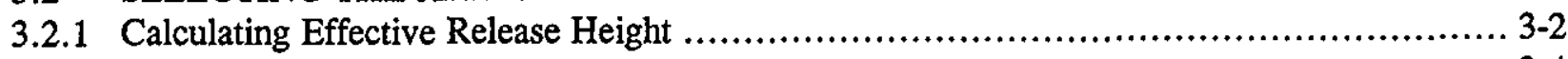

3.2.2 Using Unit Dose Factor Tables .......................................................... 3-4

3.2.3 Handling Radioactive Decay Chains ...................................................... $3-4$

3.3 RADIONUCLIDES WITHOUT UNIT DOSE FACTORS ............................... 3-5

3.4 WHEN A SCENARIO SPECIFIC CAP88-PC RUN IS WARRANTED .................... $3-6$



3.4.2 Releases that are not Chronic ............................................................. 3-6

3.4.3 Estimated Doses are Near 0.1 Millirem per Year ........................................ 3-6

3.4.4 Default Lung Retention Classes or Particle Sizes are Inappropriate .......................... 3-7

3.4.5 Releases Dominated by Volatile Radionuclides ........................................... 3-7

3.4.6 Releases from Area Sources ........................................................... $3-11$

3.4.7 Releases Dominated by Short-Lived Radionuclides ...................................... 3-11

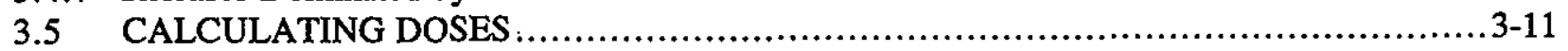

3.6 REPORTING RELEASES AND DOSES IN A NOTICE OF CONSTRUCTION.............3-14

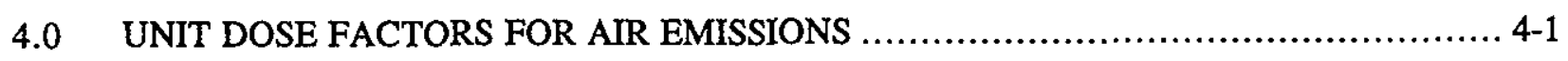

5.0 PEER REVIEW DOCUMENTATION ............................................. 5



\section{FIGURES}

Figure 4-1. Hanford Site Map of Emission Zones ................................................................................. 4-2

Figure 4-2. 300 Area Map of Emission Zones................................................................................... 4-3 


\section{TABLES}

Table 3-1. Default Lung Clearance Classes and Particle Sizes for CAP88-PC. ................................. 3-8

Table 3-2. Example 1--Decay Correction of Radionuclide Inventory. ...........................................3-12

Table 3-3. Example 1--Potential to Emit Calculation for an Elevated Release from the Western Portion of the 300 Area ................................................................................ 3-13

Table 3-4. Example 2--Potential to Emit Calculation for a Ground Level Release from the Eastern Portion of the 300 Area. ..................................................................................3 3-14

Table 3-5. Example 3--Original Assessed Potential to Emit for the Stack. ..................................... 3-15

Table 3-6. Example 3--Original Assessed Potential to Emit for the Stack's NOC............................ 3-16

Table 3-7. Example 3--Assessed Potential to Emit for the New Activity....................................... 3-16

Table 3-8. Example 3--Assessed Potential to Emit for the Stack, Including New Activity.............. 3-17

Table 3-9. Example 3--Assessed Potential to Emit for the Stack Notice of Construction................ 3-17

Table 3-10. Example 3--Detectability Check for Biweekly Sampling when the Stack Operates at 2,500 Cubic Feet per Minute.................................................................................. 3-18

Table 3-11. Example 3--Detectability Check for Monthly Sampling when the Stack Operates at 2,500 Cubic Feet per Minute................................................................................... 3-18

Table 3-12. Example 3--Detectability Check for Biweekly Sampling when the Stack Operates at 1,900 Cubic Feet per Minute. ........................................................................................ 3-19

Table 4-1. Release Locations and Meteorological Stations Used. ................................................4-4

Table 4-2. Direction and Distance to each Nearest Offsite Receptor Location from each Release



Table 4-3. 100-B/C Area--CAP88-PC Unit Dose Factors by Effective Release Height (mrem/Ci).

Table 4-4. 100-D/DR Area--CAP88-PC Unit Dose Factors by Effective Release Height (mrem/Ci).

Table 4-5. 100-F Area--CAP88-PC Unit Dose Factors by Effective Release Height

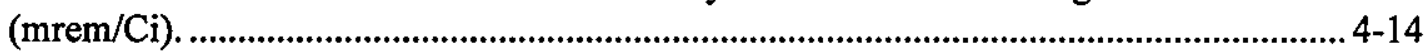

Table 4-6. 100-H Area--CAP88-PC Unit Dose Factors by Effective Release Height

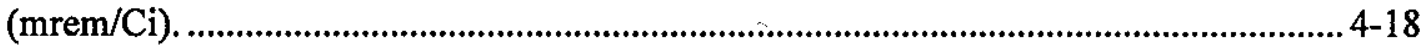

Table 4-7. 100-K Area--CAP88-PC Unit Dose Factors by Effective Release Height

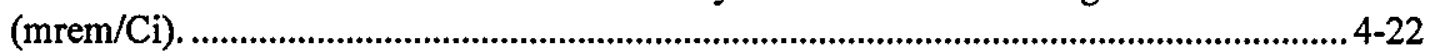

Table 4-8. 100-N Area--CAP88-PC Unit Dose Factors by Effective Release Height

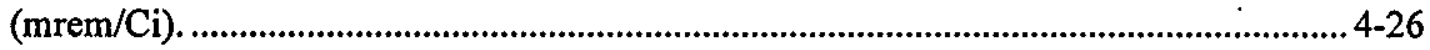

Table 4-9. 200 East Area--CAP88-PC Unit Dose Factors by Effective Release Height



Table 4-10. 200 West Area--CAP88-PC Unit Dose Factors by Effective Release Height (mrem/Ci).

Table 4-11. 300 East Area--CAP88-PC Unit Dose Factors by Effective Release Height (mrem/Ci)

Table 4-12. 300 West Area--CAP88-PC Unit Dose Factors by Effective Release Height (mrem/Ci).

Table 4-13. 400 Area--CAP88-PC Unit Dose Factors by Effective Release Height (mrem/Ci). 
HNF-3602, Vol. 1

\section{GLOSSARY}

\section{ACRONYMS}

$\begin{array}{ll}\text { AED } & \text { aerodynamic equivalent diameter } \\ \text { AMAD } & \text { activity median aerodynamic diameter } \\ \text { ANSI } & \text { American National Standards Institute } \\ \text { ASME } & \text { American Society of Mechanical Engineers } \\ \text { ASTM } & \text { American Society for Testing and Materials } \\ \text { CFR } & \text { Code of Federal Regulations } \\ \text { DCG } & \text { derived concentration guides } \\ \text { DOE } & \text { U.S. Department of Energy } \\ \text { DOE-RL } & \text { U.S. Department of Energy, Richland Operations Office } \\ \text { EPA } & \text { U.S. Environmental Protection Agency } \\ \text { ERC } & \text { Environmental Restoration Contract } \\ \text { FEMP } & \text { facility environmental monitoring plan } \\ \text { HEDOP } & \text { Hanford Environmental Dose Overview Panel } \\ \text { HEPA } & \text { high-efficiency particulate air } \\ \text { NDA } & \text { nondestructive assay } \\ \text { NOC } & \text { notice of construction } \\ \text { NOR } & \text { nearest offsite receptor } \\ \text { PHMC } & \text { Project Hanford Management Contract } \\ \text { PNNL } & \text { Pacific Northwest National Laboratory } \\ \text { PTRAEU } & \text { portable temporary radioactive air emission unit } \\ \text { STP } & \text { standard temperature and pressure } \\ \text { WAC } & \text { Washington Administrative Code } \\ \text { WDOH } & \text { Washington State Department of Health } \\ & \end{array}$

\section{DEFINITION OF TERMS}

Acute Release. A short duration release with a significant emission rate

Chronic Release. When emissions are nearly continuous and the emission rate is nearly constant for a period of at least 6 months

Emission Zone. The atmospheric dispersion factors were used to divide the Hanford Site into emission zones, which are zones where the same unit dose factors can be used for potential to emit calculations. The Hanford Site is divided up into operating areas. The emission zones are typically defined by the boundaries of each operating area except for the 300 Area, which was divided into two emission zones (300-E and 300-W).

Nearest Offsite Receptor. Nearest offsite receptor was established for each emission zone. The nearest offsite receptor is the nearest point from an emission zone within the sector providing the highest atmospheric dispersion factors for particulate, volatile, and gaseous emissions. 
HNF-3602, Vol. 1

This page intentionally left blank. 
HNF-3602, Vol. 1

\subsection{PURPOSE AND SCOPE}

The purpose of this document is to provide Hanford Site facilities a handbook for estimating potential emissions and the subsequent offsite doses. General guidelines and information are provided to assist personnel in estimating emissions for use with U.S. Department of Energy (DOE) facility effluent monitoring plans (FEMPs) and regulatory notices of construction (NOCs), per 40 Code of Federal Regulations (CFR) Part 61, Subpart H, and Washington Administrative Code (WAC) Chapter 246-247 requirements. This document replaces Unit Dose Calculation Methods and Summary of Facility. Effluent Monitoring Plan Determinations (WHC-EP-0498). Meteorological data from 1983 through 1996, 13-year data set, was used to develop the unit dose factors provided by this document, with the exception of two meteorological stations. Meteorological stations 23 and 24, located at Gable Mountain and the 100-F Area, only have data from 1986 through 1996, 10-year data set.

The scope of this document includes the following:

- Estimating emissions and resulting effective dose equivalents (EDE) to a facility's nearest offsite receptor (NOR) for use with NOCs under 40 CFR Part 61, Subpart $\mathrm{H}$, requirements

- Estimating emissions and resulting EDEs to a facility's or emission unit's NOR for use with NOCs under the WAC Chapter 246-247 requirements

- Estimating emissions and resulting EDEs to a facility's or emission unit's NOR for use with FEMPs and FEMP determinations under DOE Orders 5400.1 and 5400.5 requirements.

Extreme caution should be exercised if the information contained within this document is used for purposes outside of the identified scope. The information in this document is not suitable for estimating actual emissions to demonstrate compliance with annual dose limits. Facility compliance with federal, DOE, and state requirements on radioactive airborne emissions is demonstrated through the effluent reporting programs on the Hanford Site.

The methods and information provided in this document have been reviewed and approved by a Hanford Environmental Dose Overview Panel (HEDOP) reviewer. The Hanford Environmental Dose Overview Panel was chartered, in 1990, by U.S. Department of Energy, Richland Operations Office (DOE-RL) to:

- Ensure that appropriate radiological and nonradiological environmental and health dose assessment methods are used on the Hanford Site

- Ensure that all Hanford Site related environmental and health dose assessments technically are consistent

- Foster communication among the contractors regarding environmental and health dose assessments.

The scope of HEDOP covers all environmental and health dose assessments of potential or actual exposures to onsite and offsite receptors, that involve the environmental transport of potentially harmful material that could be or has been released from an onsite operation or facility. 
HNF-3602, Vol. 1

This page intentionally left blank. 


\subsection{ESTIMATING POTENTIAL EMISSIONS}

There are many acceptable methods for estimating potential emissions. This document describes the methods that currently are accepted by or previously have been approved by the Washington State Department of Health (WDOH) and the U.S. Environmental Protection Agency's (EPA) Region 10. Methods different from those described in the following sections also might be acceptable, but most likely will require a more extensive review and approval cycle. The same methods for estimating an emission unit's potential emissions for a NOC should be used for the FEMP and the FEMP determination.

\subsection{PRE-APPROVED METHODS}

The following four methods for estimating potential emissions are approved for use by WAC 246-247-30(21):

1. Multiplying the annual possession quantity of each radionuclide by the release fractions provided in WAC 246-247. (Note: This is the same procedure provided in 40 CFR Part 61, Appendix D)

2. Back calculations using measured emission rates and in situ measurements of the control device efficiencies

3. Measure the quantities of radionuclides captured in the control device, coupled with in situ measurements of the control equipment efficiencies

4. Sample the effluent upstream from all control devices.

WAC 246-247-30 (21)(e) and 40 CFR Part 61, Subpart H, Section 61.96, also allows the use of alternative methods, which must be approved by the WDOH and EPA Region 10, before using these methods.

WDOH and EPA Region 10 previously approved the use of the following alternative method for estimating potential emissions:

5. Multiplying the annual possession quantity of each radionuclide by a material-specific spill release fractions, rather than using the release fractions in WAC 246-247 and 40 CFR Part 61, Appendix D.

40 CFR Part 61, Subpart H, authorizes the use of Method 1 for calculating potential emissions. EPA Region 10 previously approved the use of Methods 2 through 5 as alternative methods.

The use of methods other than Methods 1 through 5 usually require a more extensive review and approval process. However, the use of previously approved methods should not require a review and approval cycle as extensive as a method that uses a new technology or methodology.

\subsubsection{Method 1}

Method 1 is the prescribed method in 40 CFR Part 61, Appendix D, and WAC 246-247-030(21). The two methods described by the regulations are almost identical, with the exception of a few minor differences. The following text states the method as contained in WAC 246-247-030(21)(a).

"Multiply the annual possession quantity of each radionuclide by the release fraction for that radionuclide, depending on its physical state. Use the following release fractions: 
(i) 1 for gases;

(ii) $10^{-3}$ for liquids or particulate solids; and

(iii) $10^{-6}$ for solids.

Determine the physical state for each radionuclide by considering its chemical form and the highest temperature to which it is subjected. Use a release fraction of one if the radionuclide is subjected to temperatures at or above its boiling point; use a release fraction of $10^{-3}$ if the radionuclide is subjected to temperatures at or above its melting point, but below its boiling point. If the chemical form is not known, use a release fraction of one for any radionuclide that is heated to a temperature of 100 degrees Celsius or more, boils at a temperature of 100 degrees Celsius or less, or is intentionally dispersed into the environment".

This method is extremely conservative, because many materials have release fractions for accident scenarios that are orders of magnitude less than those provided in Appendix D. For example, an accident scenario for a spill of powder from a 1 meter height, a 4 E-05 release fraction (Sutter et al. 1981) would be used when the particle size is 10 micron aerodynamic equivalent diameter (AED) and less. Likewise, 4 E-05 release fraction (Sutter et al. 1981) would be used for a liquid spill from a 3 meter height. The release fractions for both of these cases are more than two orders of magnitude less than the Appendix D release fraction of $1 \mathrm{E}-03$ for particulate solids.

When the properties of the source term(s) are known and alternate release fractions are available, the use of Method 5 should be considered.

\subsubsection{Method 2}

WAC 246-247-030 (21)(b) states that with approval a back-calculation using measured emission rates and in situ measurements of the control equipment efficiencies can be used to estimate potential emissions.

Control equipment efficiencies should be obtained using the methods prescribed by ASME/ANSI N509, Testing of Nuclear Air Treatment Systems.

Most of the emission control equipment on the Hanford Site consists of a high-efficiency particulate air (HEPA) filter system. The Nuclear Air Cleaning Handbook (ERDA 76-21) provides a decontamination factor of $3000^{\text {n }}$ for HEPA filter systems, whereas ' $n$ ' represents the number of HEPA filters in series.

The use of a decontamination factor of $3000^{\mathrm{n}}$ has been allowed by WDOH and EPA for systems using $\mathrm{n}$ banks of HEPA filters in series. The potential emissions are calculated by multiplying the actual annual emissions by the decontamination factor $\left(3000^{\mathrm{n}}\right)$.

Method 2 can be extremely conservative for a contaminated system. When processing no longer occurs, the resuspension of contamination downstream of the HEPA filters can dominate the airborne releases from a facility. Multiplying these releases by $3000^{\mathrm{n}}$ can overestimate the potential emissions by an order or more of magnitude (Barnett and Davis 1996). 
HNF-3602, Vol. 1

\subsubsection{Method 3}

WAC 246-247-030(21)(c) states that, with approval a measurement of the quantities of radionuclides captured in the control device, coupled with in situ measurements of the control equipment, efficiencies can be used to estimate potential emissions. There are several variations of performing this method. Each variation is described in the following text.

\subsubsection{Method 3.1}

Samples of the collection media are obtained from the control device, such that the samples are representative of the radionuclide inventory contained within the control device. Isotopic analyses are performed on the samples. The radionuclide inventory in the control device is estimated using the sample results, operating history for the device, and the appropriate radioactive decay corrections. An annual release rate is calculated using the radionuclide inventory of the control device, operating history, and collection efficiency. The potential emissions are calculated by dividing the annual release rate by the collection efficiency of the control device.

\subsubsection{Method 3.2}

A nondestructive assay measurement (NDA) is used to determine the radionuclide inventory of the control device. The radionuclide inventory in the control device is estimated using the NDA results, operating history for the device, and the appropriate radioactive decay corrections. An annual release rate is calculated using the radionuclide inventory of the control device, operating history, and collection efficiency. The potential emissions are calculated by dividing the annual release rate by the collection efficiency of the control device.

The NDA method most frequently used on the Hanford Site (WHC-SD-ER-TP-004) uses either a sodium iodide or high purity germanium gamma ray detector to quantify the gamma emitting radionuclides collected by the control device. An annual release rate is calculated for each gamma emitting radionuclide. Annual release rates for non-gamma emitting radionuclides are calculated by using ratios obtained from actual radionuclide emissions measurements or inventory data.

\subsubsection{Method 3.3}

Either samples from the collection media or NDA measurements are used to determine the radionuclide inventory of the control device. The radionuclide inventory in the control device is estimated using the sample or NDA results, operating history for the device, and the appropriate radioactive decay corrections. An annual release rate is calculated using the radionuclide inventory of the control device and its operating history. The potential emissions are calculated by adding the annual release rate, determined from the control device, to the actual annual emissions.

\subsubsection{Method 4}

WAC 246-247-030(21)(d) states that, with approval, sampling the effluent upstream from all control devices can be used to estimate potential emissions.

Representative air samples are collected at a location upstream from all control devices. The samples are analyzed for the expected radionuclides, yielding concentrations of radionuclides in the effluent stream. The potential emissions are calculated by multiplying the radionuclide concentrations by the annual discharge volume. 


\subsubsection{Method 5}

WAC 246-247-030(21)(a) states that, with approval, other release fractions may be used to estimate potential emissions.

This method is identical to Method 1 with the exception of material-specific release fractions being used to calculate potential emissions instead of the release fractions provided in WAC 246-247-030 or 40 CFR Part 61, Appendix D.

\subsection{METHODS NOT PRE-APPROVED}

Methods and technology used to determine potential emissions that previously have not been reviewed or approved by WDOH and EPA usually will require additional time for the regulatory agency to review and approve the new method or technology.

The use of any new methods or technology will require the review and subsequent approval of the appropriate primary Hanford Site contractor (i.e., Project Hanford Management Contract (PHMC) Fluor Daniel Hanford, Environmental Restoration Contract (ERC) - Bechtel Hanford, or PNNL - Battelle Northwest). Developing and using a new method or technology usually results in a greater cost in performing potential to emit calculations. The use of the five previously approved methods should be evaluated before using a new method or technology. 
HNF-3602, Vol. 1

\subsection{CALCULATING OFFSITE DOSES TO THE NEAREST RECEPTOR}

The method presented simplifies offsite dose calculations by eliminating the need to run the CAP88-PC software (Parks 1992). Such computer runs require familiarity with modeling assumptions used on the Hanford Site, in addition to site-specific wind data and various pathway parameters needed as input to the CAP88-PC software. This simplified method leads to doses that are not much more conservative than obtained using the software to model a specific scenario.

The components of the offsite dose calculation are discussed at length in the following sections. The first section deals with the radionuclide source term. The second section deals with the selection of unit release dose factors, and the third section describes how to combine these two into an offsite dose, and what this offsite dose means.

Variations in wind data over time are relatively minor compared with other uncertainties in the dose calculations. The emphasis placed on using a particular set of meteorological data is unlikely to have a significant impact on the overall conclusions of these calculations. It is more appropriate to use long-term average meteorological data sets for prospective potential to emit calculations, whereas, it is more appropriate to use annually averaged meteorological data for the annual regulatory compliance reporting. The longer term meteorological data are a more representative prediction of the meteorological conditions for activities that are projected several years into the future.

The dose factors contained in this document should be reviewed periodically and updated when warranted. When annual meteorological conditions change significantly, it is recommended that the dose factors be updated. The dispersion factors generated by CAP88-PC will be checked every 3 to 5 years using an updated meteorological data set. When newly generated dispersion factors vary by more than a factor of two from the dispersion factors used to generate these unit dose factors, the unit dose factors in this document will need to be updated.

\subsection{CALCULATING RELEASES}

Care should be taken to provide results with the correct number of significant digits. Significant digits should be handled in accordance with American Society for Testing and Material's (ASTM) Standard Practice for Using Significant Digits in Test Data to Determine Conformance with Specifications (ASTM E 29). To minimize round-off errors, as many significant figures as possible should be carried through all calculations until the end of the calculation. Otherwise rounding errors might be incurred. It is recommended that a computerized spreadsheet be used for all release calculations, because a computerized spreadsheet carries many digits but only displays the number of significant digits specified for the cell of the spreadsheet. No doses to the NOR should have more than two significant digits, because the unit dose factors are at best only accurate to two significant digits, even though CAP88-PC calculates doses with three significant digits.

When releases are estimated from an inventory of radioactive material, the inventory activity of each radionuclide should be corrected for radioactive decay. The date when the inventory was determined should be known, such that the inventory can be corrected for the date that the calculations were performed. If this correction is not performed, significant errors can be incurred in the calculations. The shorter the half-life of the radionuclide the larger the error will be when a correction for radioactive decay is not performed. The following are the basic equations for radioactive decay. 
Equation 3.1:

$$
\lambda=\frac{\ln 2}{\mathrm{~T}_{\frac{1}{2}}}
$$

Equation 3.2:

$$
A(t)=A(0) e^{-\lambda t}
$$

$$
\begin{aligned}
& \lambda \text { - radioactive decay constant, } \mathrm{s}^{-1}, \mathrm{~min}^{-1}, \mathrm{hr}^{-1}, \text { day-1, or } \mathrm{yr}^{-1} \text {. } \\
& \mathrm{T}_{1 / 2} \text { - half-life of radionuclide, } \mathrm{s}, \mathrm{min}, \mathrm{hr} \text {, day, or } \mathrm{yr} \text {. } \\
& \mathrm{A}(\mathrm{t}) \text { - current activity of a radionuclide at time, } \mathrm{t}, \mathrm{Ci} \text { or } \mathrm{Bq} . \\
& \mathrm{A}(0) \text { - initial activity of a radionuclide, } \mathrm{Ci} \text { or } \mathrm{Bq} . \\
& \mathrm{t}
\end{aligned}
$$

Miscellaneous radioactive decay data (e.g. half-lives, branching ratios, decay chains, mode of decay) for most radionuclides can be found in Radioactive Decay Data Tables (DOE/TIC-11026). Equations for decay chains can be found in many textbooks.

\subsection{SELECTING THE APPROPRIATE UNIT DOSE FACTORS}

Selecting the appropriate unit dose factors is extremely important. This section provides guidance on determining effective release height, using the unit dose factor tables, and how to handle radioactive decay chains.

\subsubsection{Calculating Effective Release Height}

The effective release height is needed to use the unit dose factor tables in Section 4.0. In most cases this is only the physical height above grade. However, when dealing with stacks several other parameters must be considered.

Plume rise significantly can increase the effective stack height, which, when it is not accounted for, can result in significant errors. The plume rise attributed to momentum and buoyancy needs to be added to the physical stack height to obtain the effective release height (Equation 3.3).

\section{Equation 3.3:}

$$
\mathrm{H}_{\mathrm{eff}}=\mathrm{H}+\Delta \mathrm{h}_{\mathrm{m}}+\Delta \mathrm{h}_{\mathrm{b}}
$$

$$
\begin{aligned}
& \mathrm{H}_{\text {eff }} \text { - effective release height for stacks, m. } \\
& \mathrm{H} \text { - physical stack height (height above grade), } \mathrm{m} \text {. } \\
& \Delta \mathrm{h}_{\mathrm{m}} \text { - plume rise attributable to momentum, m. } \\
& \Delta \mathrm{h}_{\mathrm{b}} \text { - plume rise attributable to buoyancy, m. }
\end{aligned}
$$

The plume rise attributable to momentum can be determined using Equation 3.4 or Equation 3.5. Equation 3.5 is a simplified version of Equation 3.4, which saves the user from calculating the stack velocity. 
Equation 3.4:

$$
\Delta \mathrm{h}_{\mathrm{m}}=\frac{1.5 \mathrm{vd}}{\mu}
$$



The following equation is a simplified equation for $\Delta \mathrm{h}_{\mathrm{m}}$ when the stack flow rate and the stack diameter are known in the units of measurement that are specified. This equation includes all unit conversion factors and uses $3.4 \mathrm{~m} / \mathrm{s}(7.6 \mathrm{mph})$ for the average wind velocity $(\mu)$.

Equation 3.5:

$$
\Delta h_{m}=(2.65 E-04) \frac{F_{S T P}}{d}
$$

$$
\begin{aligned}
& \Delta \mathrm{h}_{\mathrm{m}} \text { - plume rise attributable to momentum, } \mathrm{m} \text {. } \\
& \mathrm{d} \text { - inside stack diameter, } \mathrm{m} \text {. } \\
& \mathrm{F}_{\text {STP }} \text { - volumetric stack flow rate at STP, cfm. }
\end{aligned}
$$

Equations 3.6 through 3.9 can be used to calculate the plume rise attributable to plume buoyancy. Note that CAP88-PC uses several equations for buoyant plume rise. These equations apply to NOR locations greater than 10 times the physical stack height and atmospheric stability classes A, B, C, and D. This is the only buoyant plume rise equation provided in this document, because it is the most appropriate equation for virtually every application that might be encountered on the Hanford Site. Equation 3.9 is a simplified version of Equations 3.6 through 3.8, which saves the user several calculational steps.

Equation 3.6:

$$
\Delta \mathrm{h}_{\mathrm{b}}=\frac{1.6\left(3.7 \mathrm{E}-05 \mathrm{Q}_{\mathrm{H}}\right)^{\frac{1}{3}}(10 \mathrm{H})^{\frac{2}{3}}}{\mu}
$$
$\Delta \mathrm{h}_{\mathrm{b}}$ - plume rise attributable to buoyancy, $\mathrm{m}$.
$\mathrm{Q}_{\mathrm{H}} \quad$ - heat emission from stack, cal/sec.
$\mathrm{H}$ - physical stack height (height above grade), $\mathrm{m}$.
$\mu \quad$ - average wind velocity, $\mathrm{m} / \mathrm{s}$, use $3.4 \mathrm{~m} / \mathrm{s}$ (7.6 mph) (PNNL-11794).

Equation 3.7:

$$
\mathrm{Q}_{\mathrm{H}}=8.48 \mathrm{E}+04 \mathrm{~F}_{\mathrm{a}}\left(\frac{\mathrm{T}_{\mathrm{o}}}{\mathrm{T}_{\mathrm{a}}}-1\right)
$$

$\mathrm{Q}_{\mathrm{H}} \quad$ - heat emission from stack, cal/sec.

$\mathrm{T}_{\mathrm{a}} \quad$ - ambient temperature, $\mathrm{K}$, use $285.2 \mathrm{~K}\left(12.0^{\circ} \mathrm{C}\right)(\mathrm{PNNL}-11794)$.

$T_{0} \quad-$ exit temperature of the stack gas, $K$.

$\mathrm{F}_{2} \quad$ - volumetric stack flow rate at $\mathrm{T}_{2}, \mathrm{~m}^{3} / \mathrm{s}$. 
Equation 3.8:

$$
F_{\mathrm{a}}=F_{S T P} \sqrt{\frac{T_{a}}{T_{S T P}}}=0.9948 F_{S T P}
$$

$\mathrm{F}_{\mathrm{a}}$ - volumetric stack flow rate at $\mathrm{T}_{\mathrm{a}}, \mathrm{m}^{3} / \mathrm{s}$.

$\mathrm{F}_{\mathrm{STP}}$ - volumetric stack flow rate at $\mathrm{STP}, \mathrm{m}^{3} / \mathrm{s}$.

$\mathrm{T}_{\mathrm{a}} \quad$ - ambient temperature, $\mathrm{K}$, use $285.2 \mathrm{~K}\left(12.0^{\circ} \mathrm{C}\right)(\mathrm{PNNL}-11794)$.

$\mathrm{T}_{\mathrm{s}} \quad$ - standard temperature, $\mathrm{K}, 288.2 \mathrm{~K}\left(15.0^{\circ} \mathrm{C}\right)$.

The following equation is a simplified equation for $\Delta \mathrm{h}_{\mathrm{b}}$ when the physical stack height, stack flow rate, and stack temperature are known in the units of measurement that are specified. This equation includes all unit conversion factors and uses $3.4 \mathrm{~m} / \mathrm{s}(7.6 \mathrm{mph})$ for average wind velccity $(\mu)$.

Equation 3.9:

$$
\Delta \mathrm{h}_{\mathrm{b}}=2.50 \mathrm{E}-01 \mathrm{H}^{\frac{2}{3}}\left[\mathrm{~F}_{\mathrm{STP}}\left(\frac{\mathrm{T}_{\mathrm{o}}}{285.2}\right)\right]^{\frac{1}{3}}
$$

$$
\begin{aligned}
& \Delta \mathrm{h}_{\mathrm{b}} \text { - plume rise attributable to buoyancy, m. } \\
& \mathrm{F}_{\text {STP }} \text { - volumetric stack flow rate at STP, cfm. } \\
& \mathrm{H} \text { - physical stack height (height above grade), } \mathrm{m} \text {. } \\
& \mathrm{T}_{0} \text { - exit temperature of the stack gas, } \mathrm{K} \text {. }
\end{aligned}
$$

\subsubsection{Using Unit Dose Factor Tables}

Unit dose factors have been calculated for each emission zone. Emission zones are essentially the major operating areas on the Hanford Site with the exception of the 300 Area, which has been divided into two emission zones. The appropriate emission zone should be determined, such that correct set of unit dose factors are used. Figures 4-1 and 4-2 provide maps of the emission zones. The dividing line for the zones within the 300 Area is the dashed line in Figure 4-2. The dividing line for the 300 Area is represented by the western boundary for the 300-FF-1 Operable Unit and George Washington Way.

The unit dose factors in each table are organized by effective release height and radionuclide. Two effective release heights were used to represent both ranges of effective release heights. Ground level releases (i.e., any releases with an effective release height $<40 \mathrm{~m}$ ) were modeled using an effective release height of 10 meters. Elevated releases (i.e., any releases with an effective release height $\geq 40 \mathrm{~m}$ ) were modeled using an effective release height of 40 meters. Section 3.2.1 provides specific guidance on calculating the effective release height. Nuclides in the tables are arranged in order of atomic number and atomic mass.

Once one determines the emission zone that an emission unit is in and its effective release height, one can go to the unit dose factor tables in Section 4.0 and look up the unit dose factors for each nuclide.

\subsubsection{Handling Radioactive Decay Chains}

Many radionuclides decay into other radionuclides, creating a radioactive decay chain consisting of a parent nuclide and its progeny (i.e., daughter(s) or decay product(s)). The unit dose factors provided in 
Section 4.0 do not include any of the progeny for nuclides with decay chains. Each nuclide in a source term should be evaluated to determine if there are any decay products that could be significant to the calculations. If so, the source term should be adjusted to include the significant decay products. The unit dose factors for each of the parent nuclides and each of the decay products would be used to calculate the total dose.

Often the progeny are excluded from inventories, especially if it requires considerable time for significant ingrowth of the progeny to occur. Sometimes the progeny are inadvertently excluded, even when the period for significant ingrowth is very short. An example of this is Y-90, the progeny of Sr-90, which inadvertently is excluded from many inventories and calculations. A source term of pure Sr-90 only requires 19 days for $\mathrm{Y}-90$ to reach secular equilibrium with $\mathrm{Sr}-90$, such that there are equal quantities, in terms of radioactivity, present. Secular equilibrium is possible when the half-life of the parent nuclide is much greater than progeny. The time required for the immediate decay product to reach secular equilibrium with its parent nuclide is approximately seven times the half-life of the decay product.

Branching ratios need to be accounted for as well. Nuclides that can decay into more than one decay product have branching ratios. The branching ratio is the percentages of the radioactive decays that transform into a particular decay product. An example of this is Cs-137, which decays to $\mathrm{Ba}-137 \mathrm{~m}$ 94.6 percent of the time and decays to stable Ba-137 5.4 percent of the time. Because of the 94.6 percent branching ratio, the activity of $\mathrm{Ba}-137 \mathrm{~m}$ will come within 94.6 percent of the activity of $\mathrm{Cs}-137$, within approximately seven half-lives (18 minutes).

Because of branching ratios and different half-lives, longer decay series are much more difficult to perform radioactive decay calculations. It is recommended that a computer code be used for most radioactive decay calculations, especially when there are multiple decay products for a nuclide.

\subsection{RADIONUCLIDES WITHOUT UNIT DOSE FACTORS}

CAP88-PC only calculates doses for the 265 radionuclides contained in its library files. If no dose factors were computed for a particular nuclide, the dose factors for another nuclide could be substituted for the nuclide not included in the CAP88-PC Library. Selection of an appropriate substitute nuclide is very important, such that nonconservative errors and grossly conservative errors can be avoided.

The substitute nuclide should have similar physical characteristics (i.e., particulate, volatile, or gaseous) to the missing nuclide, such that differences attributable to atmospheric dispersion are minimized. It is recommended that a substitute nuclide be chosen such that the substitute nuclide would provide a more conservative unit dose factor. Note that nuclides such as $\mathrm{Sr}-90, \mathrm{Cs}-137, \mathrm{Pu}-239$, and Am-241 always might not be suitable substitutes, because the more exotic nuclides can sometimes yield a more significant dose. Similarly, these nuclides also can result in grossly conservative errors that should be avoided when possible.

There are three methods that are recommended for selecting a substitute nuclide. The first method would be to find a nuclide with the same physical characteristics and comparable derived concentration guides (DCGs) for air. A table of the DCGs can be found in Chapter III of DOE Order 5400.5. The second method would be to find a nuclide with the same physical characteristics and a comparable unit dose factor calculated using a generic run of the GENII environmental dose assessment code (PNL 6584). The third method would use both methods to select a suitable radionuclide. 
HNF-3602, Vol. 1

\subsection{WHEN A SCENARIO SPECIFIC CAP88-PC RUN IS WARRANTED}

This section describes most of the scenarios where it would be inappropriate to use these unit dose factors and when unit dose factors for a specific scenario should be generated.

\subsubsection{Emission Unit not Within any Emission Zone}

When an emission unit is not located within or near a given emission zone, as indicated in Figures 4-1 and 4-3, CAP88-PC will need to be run for the specific scenario. One exception to this would be emission units between the 200 East and 200 West emission zones. If an emission unit is located between these emission zones, the unit dose factors for the zone the emission unit is closest to should be used. In the event the emission unit is almost exactly between the 200 East and 200 West emission zones, the unit dose factors yielding the most conservative total dose should be used.

\subsubsection{Releases that are not Chronic}

Potential release (potential-to-emit) calculations usually are performed for chronic release scenarios, when emissions nearly are continuous and the emission rate nearly is constant for relatively long periods. A chronic release is defined as nearly continuous for a period of at least 6 months for the purposes of this document. However, some of the release scenarios on the Hanford Site are for relatively short periods ( $<6$ months), can be intermittent, and/or can have variable emission rates. These types of releases are not considered chronic releases, and the unit dose factors as provided in Section 4.0 are inappropriate.

The CAP88-PC model was designed to only address chronic releases. Currently, there are no regulatory mandated or approved atmospheric dispersion and dose modeling codes that can model releases that are not chronic. Using CAP88-PC generated unit dose factors for these scenarios significantly CAN underestimate doses to the NOR.

When potential to emit calculations are needed for a scenario involving a release that is not chronic, it is recommended that unit dose factors be adjusted. Observations have been made using the GENII model that dispersion factors for short-term releases vary by the ratio of 365 days divided by the time of the release from the chronic dispersion factors. As a result, doses for short-term releases can be calculated by using chronic unit doses that have been adjusted. To obtain an adjustment factor, the number of days in the year ( 365 days) should be divided by the number of days the emission unit is expected to operate during a given calendar year. Each of the unit dose factors should be multiplied by the adjustment factor. Note that this method should not be used for releases that have a duration of less than 1 month.

Another option is to use the GENII model to generate scenario-specific unit dose factors for short-term releases, and even when the release has a duration of less than 1 month. GENII should be used to generate unit dose factors at the 95th percentile. The GENII model is the official Hanford Site model for demonstrating compliance with DOE requirements. However, to use the GENII model or any other model for regulatory work, approval(s) from the appropriate regulatory agency(ies) is required.

\subsubsection{Estimated Doses are Near 0.1 Millirem per Year}

When estimated doses are within an order of magnitude of 0.1 millirem per year ( 1.0 to 0.01 millirem per year), a scenario specific CAP88-PC run might be warranted to eliminate additional conservatism from 
the dose calculation. The benefits of a scenario-specific run should be weighed carefully, because such a run can significantly increase the cost of performing and documenting the potential to emit.

\subsubsection{Default Lung Retention Classes or Particle Sizes are Inappropriate}

When the default lung retention class or particle for a nuclide is not appropriate and results in an unacceptably conservative or nonconservative unit dose factor, a scenario-specific run should be considered. However, to consider a scenario-specific run, the lung retention class and/or the particle size in terms of activity median aerodynamic diameter (AMAD) must be known. The lung retention classes for many compounds can be found in Chapter III of DOE Order 5400.5.

CAP88-PC only allows the user to toggle between the valid choices for lung clearance class and particle size. Options for particle size are extremely limited by CAP88-PC, such that most nuclides can not be changed from their default setting. The options for lung clearance class are very limited also.

CAP88-PC limits the user to certain lung clearance classes that it considers as valid, even when the other valid lung clearance classes exist. The default lung clearance class and particle size used by CAP88-PC for each nuclide are provided in Table 3-1. Note that CAP88-PC does not specify the default lung clearance class or allows the user to specify a class for some nuclides. Also, CAP88-PC specifies a particle size of 0.0 for some nongaseous nuclides and does not allow the user to change the default parameter.

If a user encounters a scenario that might significantly underestimate or overestimate the dose because of the limitations CAP88-PC has imposed on the user for lung clearance classes and particle sizes, the user might wish to seek approval from the regulatory agency(ies) to use a more appropriate computer model.

\subsubsection{Releases Dominated by Volatile Radionuclides}

When developing the unit dose factors, an observation regarding the scenarios using volatile nuclides (i.e. halogen nuclides) was made. The distances and directions to the NOR were different for volatile nuclides in the 100-B/C, 100-D/DR, 100-H, 100-K, 100-N, and 200 West Area emission zones. A single NOR was chosen for each emission zone, resulting in an acceptable amount of error for most scenarios. However, the error might become unacceptable when releases are dominated by volatile nuclides.

A scenario-specific run of CAP88-PC should be considered when an emission unit is located in one of the previously listed emission zones and the calculated unabated dose(s) attributable to any specific volatile nuclide accounts for 5 percent or more of the total dose for that emission source, using the unit dose factors provided in Section 4.0. This does not apply to the 100-F, 200 East, 300 East, 300 West, and 400 Areas emission zones, because the distances and directions to the NOR were the same for particulate, volatile, and gaseous nuclides. 
Table 3-1. Default Lung Clearance Classes and Particle Sizes for CAP88-PC.

\begin{tabular}{|c|c|c|c|c|c|}
\hline Nuclide & $\begin{array}{c}\text { Lung } \\
\text { clearance } \\
\text { class }^{\mathrm{a}}\end{array}$ & $\begin{array}{c}\text { Particle } \\
\text { size } \\
(\mu \mathrm{m})\end{array}$ & Nuclide & $\begin{array}{c}\text { Lung } \\
\text { clearance } \\
\text { class }^{\mathrm{a}}\end{array}$ & $\begin{array}{c}\text { Particle } \\
\text { size } \\
(\mu \mathrm{m})\end{array}$ \\
\hline $\mathrm{H}-3$ & N/A & 0.00 & $\mathrm{Kr}-85$ & N/A & 0.00 \\
\hline $\mathrm{Be}-7$ & $\mathrm{Y}$ & 1.00 & $\mathrm{Kr}-85 \mathrm{~m}$ & N/A & 0.00 \\
\hline $\mathrm{Be}-10$ & -- & 0.00 & $\mathrm{Kr}-87$ & N/A & 0.00 \\
\hline$C-11$ & D & 1.00 & $\mathrm{Kr}-88$ & N/A & 0.00 \\
\hline C-14 & N/A & 0.00 & $\mathrm{Kr}-89$ & N/A & 0.00 \\
\hline C-15 & D & 1.00 & $\mathrm{Kr}-90$ & N/A & 0.00 \\
\hline $\mathrm{N}-13$ & D & 1.00 & $\mathrm{Rb}-86$ & $\mathrm{D}$ & 1.00 \\
\hline $0-15$ & D & 1.00 & $\mathrm{Rb}-87$ & D & 1.00 \\
\hline F-18 & D & 1.00 & $\mathrm{Rb}-88^{\circ}$ & D & 1.00 \\
\hline $\mathrm{Na}-22$ & D & 1.00 & $\mathrm{Rb}-89$ & D & 1.00 \\
\hline $\mathrm{Na}-24$ & D & 1.00 & $\mathrm{Rb}-90$ & --- & 0.00 \\
\hline P-32 & D & 1.00 & $\mathrm{Rb}-90 \mathrm{~m}$ & $\ldots$ & 0.00 \\
\hline S-35 & D & 1.00 & St-89 & D & 1.00 \\
\hline Ar-41 & N/A & 0.00 & Sr-90 & D & 1.00 \\
\hline $\mathrm{K}-40$ & D & 1.00 & Sr-91 & D & 1.00 \\
\hline $\mathrm{Ca}-41$ & - & 0.00 & Sr-92 & D & 1.00 \\
\hline $\mathrm{Sc}-46$ & $Y$ & 1.00 & $Y-90$ & $\mathrm{Y}$ & 1.00 \\
\hline $\mathrm{Cr}-51$ & $\mathrm{Y}$ & 1.00 & Y-90m & $\cdots$ & 0.00 \\
\hline Mn-54 & $\mathbf{w}$ & 1.00 & Y-91 & $\mathbf{Y}$ & 1.00 \\
\hline Mn-56 & W & 1.00 & Y-91m & $\mathrm{Y}$ & 1.00 \\
\hline $\mathrm{Fe}-55$ & $\bar{W}$ & 1.00 & Y-92 & $\bar{Y}$ & 1.00 \\
\hline $\mathrm{Fe}-59$ & W & 1.00 & Y-93 & $Y$ & 1.00 \\
\hline $\mathrm{Co}-57$ & $\mathbf{Y}$ & 1.00 & Zr-93 & W & 1.00 \\
\hline $\mathrm{Co}-58$ & $\mathrm{Y}$ & 1.00 & $\mathrm{Zr}-95$ & W & 1.00 \\
\hline $\mathrm{Co}-60$ & $\mathrm{Y}$ & 1.00 & $\mathrm{Nb}-93 \mathrm{~m}$ & $\mathrm{Y}$ & 1.00 \\
\hline $\mathrm{Ni}-59$ & W & 1.00 & $\mathrm{Nb}-94$ & $Y$ & 1.00 \\
\hline $\mathrm{Ni}-63$ & W & 1.00 & $\mathrm{Nb}-95$ & $\mathbf{Y}$ & 1.00 \\
\hline $\mathrm{Ni}-65$ & W & 1.00 & $\mathrm{Nb}-95 \mathrm{~m}$ & $Y$ & 1.00 \\
\hline $\mathrm{Cu}-64$ & $\mathbf{Y}$ & 1.00 & $\mathrm{Nb}-97$ & $\mathbf{Y}$ & 1.00 \\
\hline $\mathrm{Zn}-65$ & $Y$ & 1.00 & $\mathrm{Nb}-97 \mathrm{~m}$ & $\mathbf{Y}$ & 1.00 \\
\hline $\mathrm{Zn}-69 \mathrm{~m}$ & $\mathbf{Y}$ & 1.00 & Mo-93 & $\cdots$ & 0.00 \\
\hline Zn-69 & $\mathbf{Y}$ & 1.00 & Mo-99 & $\mathbf{Y}$ & 1.00 \\
\hline $\mathrm{Ga}-67$ & W & 1.00 & Tc-97 & W & 1.00 \\
\hline As -76 & W & 1.00 & Tc-99 & W & 1.00 \\
\hline $\mathrm{Se}-79$ & -- & 0.00 & Tc- $99 \mathrm{~m}$ & $\mathrm{~W}$ & 1.00 \\
\hline $\mathrm{Br}-82$ & D & 1.00 & Tc-101 & ... & 0.00 \\
\hline $\mathrm{Br}-83$ & $\cdots$ & 0.00 & Ru-97 & $\mathbf{Y}$ & 1.00 \\
\hline $\mathrm{Br}-84$ & - & 0.00 & Ru-103 & $Y$ & 1.00 \\
\hline Br-85 & - & 0.00 & $R u-105$ & $Y$ & 1.00 \\
\hline $\mathrm{Kr}-83 \mathrm{~m}$ & N/A & 0.00 & $\mathrm{Ru}-106$ & $\mathrm{Y}$ & 1.00 \\
\hline $\mathrm{Rh}-103 \mathrm{~m}$ & $\mathbf{Y}$ & 1.00 & I-129 & D & 1.00 \\
\hline Rh-105 & $\mathbf{Y}$ & 1.00 & I-130 & D & 1.00 \\
\hline Rh-105m & $\mathbf{Y}$ & 1.00 & $1-131$ & D & 1.00 \\
\hline Rh-106 & $\mathbf{Y}$ & 1.00 & I-132 & D & 1.00 \\
\hline Pd-107 & $\mathbf{Y}$ & 1.00 & I-133 & D & 1.00 \\
\hline Pd-109 & $\mathbf{Y}$ & 1.00 & I-134 & D & 1.00 \\
\hline $\mathrm{Ag}-109 \mathrm{~m}$ & $Y$ & 1.00 & I-135 & D & 1.00 \\
\hline $\mathrm{Ag}-110$ & Y & 1.00 & $\mathrm{Xe}-122$ & N/A & 0.00 \\
\hline $\mathrm{Ag}-110 \mathrm{~m}$ & $\mathbf{Y}$ & 1.00 & $\mathrm{Xe}-123$ & N/A & 0.00 \\
\hline Ag-111 & $Y$ & 1.00 & $\mathrm{Xe}-125$ & N/A & 0.00 \\
\hline
\end{tabular}


HNF-3602, Vol. 1

Table 3-1. Default Lung Clearance Classes and Particle Sizes for CAP88-PC.

\begin{tabular}{|c|c|c|c|c|c|}
\hline Nuclide & $\begin{array}{c}\text { Lung } \\
\text { clearance } \\
\text { class }^{\mathrm{a}}\end{array}$ & $\begin{array}{c}\text { Particle } \\
\text { size } \\
(\mu \mathrm{m}) \\
\end{array}$ & Nuclide & $\begin{array}{c}\text { Lung } \\
\text { clearance } \\
\text { classs }\end{array}$ & $\begin{array}{c}\text { Particle } \\
\text { size } \\
(\mu \mathrm{m}) \\
\end{array}$ \\
\hline Cd-113 & --- & 0.00 & $\mathrm{Xe}-127$ & N/A & 0.00 \\
\hline Cd-113m & --- & 0.00 & $\mathrm{Xe}-131 \mathrm{~m}$ & N/A & 0.00 \\
\hline $\mathrm{Cd}-115$ & $\mathrm{Y}$ & 1.00 & $\mathrm{Xe}-133$ & N/A & 0.00 \\
\hline $\mathrm{Cd}-115 \mathrm{~m}$ & $\mathbf{Y}$ & 1.00 & $\mathrm{Xe}-133 \mathrm{~m}$ & N/A & 0.00 \\
\hline In-113m & W & 1.00 & $\mathrm{Xe}-135$ & N/A & 0.00 \\
\hline In-115 & W & 1.00 & $\mathrm{Xe}-135 \mathrm{~m}$ & N/A & 0.00 \\
\hline In-115m & W & 1.00 & $\mathrm{Xe}-137$ & N/A & 0.00 \\
\hline Sn-113 & W & 1.00 & Xe-138 & N/A & 0.00 \\
\hline Sn-123 & -- & 0.00 & Cs-134 & D & 1.00 \\
\hline $\mathrm{Sn}-125$ & W & 1.00 & Cs-134m & D & 1.00 \\
\hline Sn-126 & $\mathrm{W}$ & 1.00 & Cs-135 & $\mathrm{D}$ & 1.00 \\
\hline $\mathrm{Sb}-124$ & W & 1.00 & Cs-136 & D & 1.00 \\
\hline $\mathrm{Sb}-125$ & w & 1.00 & Cs-137 & D & 1.00 \\
\hline $\mathrm{Sb}-126$ & W & 1.00 & Cs-138 & D & 1.00 \\
\hline $\mathrm{Sb}-126 \mathrm{~m}$ & W & 1.00 & Cs-139 & -- & 0.00 \\
\hline $\mathrm{Sb}-127$ & W & 1.00 & $\mathrm{Ba}-133$ & D & 1.00 \\
\hline $\mathrm{Te}-125 \mathrm{~m}$ & W & 1.00 & $\mathrm{Ba}-133 \mathrm{~m}$ & D & 1.00 \\
\hline Te-127 & W & 1.00 & $\mathrm{Ba}-137 \mathrm{~m}$ & D & 1.00 \\
\hline $\mathrm{Te}-127 \mathrm{~m}$ & W & 1.00 & Ba-139 & D & 1.00 \\
\hline Te-129 & W & 1.00 & Ba-140 & D & 1.00 \\
\hline $\mathrm{Te}-129 \mathrm{~m}$ & W & 1.00 & $\mathrm{Ba}-141$ & -- & 0.00 \\
\hline Te-131 & W & 1.00 & $\mathrm{Ba}-142$ & -- & 0.00 \\
\hline $\mathrm{Te}-131 \mathrm{~m}$ & W & 1.00 & La-140 & W & 1.00 \\
\hline Te-132 & W & 1.00 & La-141 & -- & 0.00 \\
\hline $\mathrm{Te}-133$ & -- & 0.00 & La-142 & - & 0.00 \\
\hline Te-133m & -- & 0.00 & Ce-141 & $\mathrm{Y}$ & 1.00 \\
\hline Te-134 & -- & 0.00 & Ce-143 & $\mathrm{Y}$ & 1.00 \\
\hline $\mathrm{I}-122$ & D & 1.00 & Ce-144 & $\mathrm{Y}$ & 1.00 \\
\hline $1-123$ & D & 1.00 & Pr-143 & $\mathrm{Y}$ & 1.00 \\
\hline I-125 & D & 1.00 & Pr-144 & $\mathrm{Y}$ & 1.00 \\
\hline $\mathrm{Pr}-144 \mathrm{~m}$ & $\mathrm{Y}$ & 1.00 & Po-211 & $\ldots$ & 0.00 \\
\hline Nd-147 & $Y$ & 1.00 & Po-212 & w & 1.00 \\
\hline $\mathrm{Pm}-147$ & Y & 1.00 & Po-213 & W & 1.00 \\
\hline Pm-148 & $\mathbf{Y}$ & 1.00 & Po-214 & W & 1.00 \\
\hline Pm-148m & $\mathbf{Y}$ & 1.00 & Po-215 & w & 1.00 \\
\hline Pm-149 & $\mathbf{Y}$ & 1.00 & Po-216 & $\mathrm{W}$ & 1.00 \\
\hline Pm-151 & -- & 0.00 & Po-218 & W & 1.00 \\
\hline $\mathrm{Sm}-147$ & W & 1.00 & At-217 & D & 1.00 \\
\hline Sm-151 & W & 1.00 & Rn-219 & N/A & 0.00 \\
\hline Sm-153 & W & 1.00 & $\mathrm{Rn}-220$ & N/A & 0.00 \\
\hline Eu-152 & W & 1.00 & $\mathrm{Rn}-222$ & N/A & 0.00 \\
\hline $\mathrm{Eu}-152 \mathrm{~m}$ & - & 0.00 & Fr-221 & D & 1.00 \\
\hline Eu-154 & W & 1.00 & Fr-223 & D & 1.00 \\
\hline Eu-155 & W & 1.00 & Ra-223 & W & 1.00 \\
\hline Eu-156 & W & 1.00 & Ra-224 & W & 1.00 \\
\hline Gd-152 & - & 0.00 & $\mathrm{Ra}-225$ & W & 1.00 \\
\hline Tb- 160 & W & 1.00 & $\mathrm{Ra}-226$ & W & 1.00 \\
\hline Ho-166 & W & 1.00 & $\mathrm{Ra}-228$ & W & 1.00 \\
\hline $\mathrm{Ho}-166 \mathrm{~m}$ & - & 0.00 & Ac-225 & $\mathrm{Y}$ & 1.00 \\
\hline Hf-181 & $\mathrm{W}$ & 1.00 & Ac-227 & $Y$ & 1.00 \\
\hline
\end{tabular}


HNF-3602, Vol. 1

Table 3-1. Default Lung Clearance Classes and Particle Sizes for CAP88-PC.

\begin{tabular}{|c|c|c|c|c|c|}
\hline Nuclide & $\begin{array}{c}\text { Lung } \\
\text { clearance } \\
\text { class } \mathrm{a}^{\mathrm{a}}\end{array}$ & $\begin{array}{c}\text { Particle } \\
\text { size } \\
(\mu \mathrm{m})\end{array}$ & Nuclide & $\begin{array}{c}\text { Lung } \\
\text { clearance } \\
\text { class } \mathrm{s}^{\mathrm{a}}\end{array}$ & $\begin{array}{c}\text { Particle } \\
\text { size } \\
(\mu \mathrm{m})\end{array}$ \\
\hline W-181 & D & 1.00 & Ac-228 & $\mathbf{Y}$ & 1.00 \\
\hline W-185 & D & 1.00 & Th-227 & $\mathbf{Y}$ & 1.00 \\
\hline W-187 & D & 1.00 & Th-228 & $\mathrm{Y}$ & 1.00 \\
\hline $\operatorname{Re}-187$ & W & 1.00 & Th-229 & $\mathrm{Y}$ & 1.00 \\
\hline Ir -192 & $Y$ & 1.00 & Th-230 & $\mathrm{Y}$ & 1.00 \\
\hline $\mathrm{Hg}-203$ & W & 1.00 & Th-231 & $\mathbf{Y}$ & 1.00 \\
\hline Tl-207 & D & 1.00 & Th-232 & Y & 1.00 \\
\hline Tl-208 & D & 1.00 & Th-234 & $\mathrm{Y}$ & 1.00 \\
\hline Tl-209 & D & 1.00 & $\mathrm{~Pa}-231$ & $\mathrm{Y}$ & 1.00 \\
\hline $\mathrm{Pb}-209$ & D & 1.00 & $\mathrm{~Pa}-233$ & Y & 1.00 \\
\hline $\mathrm{Pb}-210$ & $\mathrm{D}$ & 1.00 & $\mathrm{~Pa}-234$ & $\bar{Y}$ & 1.00 \\
\hline $\mathrm{Pb}-211$ & D & 1.00 & $\mathrm{~Pa}-234 \mathrm{~m}$ & Y & 1.00 \\
\hline $\mathrm{Pb}-212$ & D & 1.00 & U-232 & $\mathrm{Y}$ & 1.00 \\
\hline $\mathrm{Pb}-214$ & D & 1.00 & U-233 & $\mathrm{Y}$ & 1.00 \\
\hline $\mathrm{Bi}-210$ & W & 1.00 & U-234 & Y & 1.00 \\
\hline Bi-211 & W & 1.00 & U-235 & $\mathbf{Y}$ & 1.00 \\
\hline $\mathrm{Bi}-212$ & W & 1.00 & U-236 & Y & 1.00 \\
\hline Bi-213 & W & 1.00 & U-237 & Y & 1.00 \\
\hline Bi-214 & W & 1.00 & U-238 & $\mathbf{Y}$ & 1.00 \\
\hline Po-210 & W & 1.00 & $\mathrm{U}-240$ & $Y$ & 1.00 \\
\hline Np-237 & W & 1.00 & & & \\
\hline Np-238 & W & 1.00 & & & \\
\hline Np-239 & W & 1.00 & & & \\
\hline$N p-240$ & W & 1.00 & & & \\
\hline $\mathrm{Np}-240 \mathrm{~m}$ & W & 1.00 & & & \\
\hline Pu-236 & $\mathbf{Y}$ & 1.00 & & & \\
\hline $\mathrm{Pu}-238$ & $\mathrm{Y}$ & 1.00 & & & \\
\hline Pu-239 & $\mathrm{Y}$ & 1.00 & & & \\
\hline $\mathrm{Pu}-240$ & Y & 1.00 & & & \\
\hline Pu-241 & $\mathrm{Y}$ & 1.00 & & & \\
\hline $\mathrm{Pu}-242$ & $\mathrm{Y}$ & 1.00 & & & \\
\hline Pu-243 & $\mathbf{Y}$ & 1.00 & & & \\
\hline Pu-244 & $\mathbf{Y}$ & 1.00 & & & \\
\hline Am-241 & W & 1.00 & & & \\
\hline Am-242 & W & 1.00 & & & \\
\hline Am-242m & W & 1.00 & & & \\
\hline Am-243 & W & 1.00 & & & \\
\hline $\mathrm{Cm}-242$ & W & 1.00 & & & \\
\hline $\mathrm{Cm}-243$ & W & 1.00 & & & \\
\hline $\mathrm{Cm}-244$ & w & 1.00 & & & \\
\hline $\mathrm{Cm}-245$ & W & 1.00 & & & \\
\hline $\mathrm{Cm}-246$ & W & 1.00 & & & \\
\hline $\mathrm{Cm}-247$ & W & 1.00 & & & \\
\hline $\mathrm{Cm}-248$ & W & 1.00 & & & \\
\hline Cf-252 & $\mathbf{Y}$ & 1.00 & & & \\
\hline
\end{tabular}

" N/A - not applicable; D - days; W - weeks; Y - years; "--." - no data provided by CAP88-PC. 


\subsubsection{Releases from Area Sources}

When the distance/diameter ratio for an area source is 2.5 or greater, CAP88-PC models this as a point source. However, the differences in the unit dose factors are insignificant (i.e. within a factor of two) even when the ratio is as little as 0.22 . A scenario specific CAP88-PC run is recommended when the distance/diameter ratio is less than 0.22 .

Equation 3.10:

$$
\begin{aligned}
& \qquad \mathrm{d}_{\text {eff }}=\sqrt{\frac{4 \mathrm{~A}}{\pi}} \\
& \mathrm{d}_{\text {eff }} \quad-\text { effective diameter of an area source, } \mathrm{m} . \\
& \mathrm{A} \text { - area of the source, } \mathrm{m}^{2} .
\end{aligned}
$$

\subsubsection{Releases Dominated by Short-Lived Radionuclides}

When developing the unit dose factors, an observation regarding the scenarios using short-lived nuclides (i.e. nuclides with short half-lives) was made. The distances and directions to the NOR were different for short-lived nuclides in the 100-D/DR, 100-F, 100-H, and 100-N Areas emission zones. A single NOR was chosen for each emission zone, resulting in an acceptable amount of error for most scenarios. However, the error could become unacceptable when releases are dominated by short-lived nuclides.

A scenario specific run of CAP88-PC should be considered when an emission unit is located in one of the previously listed emission zones and the calculated unabated dose(s) attributable to any specific short-lived nuclide accounts for 5 percent or more of the total dose for that emission source, using the unit dose factors provided in Section 4.0. This does not apply to the 100-B/C, 100-K, 200 East, 300 East, 300 West, and 400 Areas emission zones, because the distances and directions to the NOR were the same for short-lived and long-lived nuclides.

\subsection{CALCULATING DOSES}

The unit dose factors in this document were calculated using CAP88-PC, which is a model for chronic release scenarios (i.e., nearly continuous for a period of at least 3 months). These unit dose factors are not appropriate for scenarios with releases that are acute. The user should refer to Section 3.4 .2 when a release is not considered chronic.

\section{EXAMPLE 1:}

The potential to emit is calculated using Method 1 for a facility located at least 250 meters from the Columbia River in the 300 Area. Emissions are ventilated at 2,000 cubic feet per minute $(0.944$ cubic meters per second) from a 0.1524 -meter diameter stack that is 25 meters above grade. The abatement technology on the stack consists of a single bank of HEPA filters. The average stack flow rate is estimated to be 2,000 cubic feet per minute ( 0.944 cubic meters per second). A facility has an inventory of liquid radioactive material, which was last determined 10 years ago. The process utilized by the facility heats the source term to a temperature of $150^{\circ} \mathrm{C}(423 \mathrm{~K})$, such that emissions leaving the stack are at an average temperature of $100^{\circ} \mathrm{C}(373 \mathrm{~K})$. The boiling point for the entire source term is $100^{\circ} \mathrm{C}$ $(373 \mathrm{~K})$. 
Table 3-2 provided the inventory from 10 years ago and the decay corrected inventory as of today, using Equations 3.1 and 3.2. Note the increase in the inventory of Am-241 due to the decay of the parent nuclide, $\mathrm{Pu}-241$.

Table 3-2. Example 1--Decay Correction of Radionuclide Inventory.

\begin{tabular}{|c|c|c|}
\hline Nuclide & $\begin{array}{c}\text { Inventory } \\
\text { determined } \\
10 \text { years ago } \\
(\mathrm{Ci})\end{array}$ & $\begin{array}{c}\text { Decay corrected } \\
\text { inventory } \\
\text { (Ci) }\end{array}$ \\
\hline $\mathrm{H}-3$ & $2.0 \mathrm{E}+01$ & $1.1 \mathrm{E}+01$ \\
\hline $\mathrm{Co}-60$ & $1.0 \mathrm{E}-03$ & $2.7 \mathrm{E}-04$ \\
\hline $\mathrm{Se}-79$ & $1.5 \mathrm{E}-04$ & $1.5 \mathrm{E}-04$ \\
\hline $\mathrm{Sr}-90$ & $3.0 \mathrm{E}-04$ & $2.4 \mathrm{E}-04$ \\
\hline $\mathrm{Y}-90$ & $3.0 \mathrm{E}-04$ & $2.4 \mathrm{E}-04$ \\
\hline $\mathrm{Zr}-95$ & $2.0 \mathrm{E}-04$ & 0.0 \\
\hline $\mathrm{Cs}-137^{3}$ & $5.0 \mathrm{E}-03$ & $4.0 \mathrm{E}-03$ \\
\hline $\mathrm{Ba}-137 \mathrm{~m}$ & $5.0 \mathrm{E}-03$ & $3.8 \mathrm{E}-03$ \\
\hline $\mathrm{Pu}-239$ & $9.0 \mathrm{E}-06$ & $9.0 \mathrm{E}-06$ \\
\hline $\mathrm{Pu}-241$ & $8.5 \mathrm{E}-04$ & $5.3 \mathrm{E}-04$ \\
\hline $\mathrm{Am}-241$ & 0.0 & $1.1 \mathrm{E}-05$ \\
\hline
\end{tabular}

Using 40 CFR 61, Appendix D, the release factor for this inventory is one, because of the source term being heated to over $100^{\circ} \mathrm{C}$. The adjustment factor for the HEPA filters is one, because the emissions are treated as a gas when above their boiling point.

Plume rise attributable to momentum and buoyancy is significant for this stack, because of the small stack diameter and high temperature of the emissions. The plume rise attributable to momentum was calculated to be 3.5 meters, using Equation 3.5. The plume rise attributable to buoyancy was calculated to be 17.4 meters, using equation 3.9. The effective stack height was calculated to be 45.9 meters, using Equation 3.3. As a result, the unit dosed factors for elevated releases need to be used, because $\mathrm{H}_{\mathrm{eff}} \geq 40$ meters, even though the physical stack height is only 25 meters.

Table 3-3 provides the potential to emit data and subsequent doses to the NOR for Example 1. 
Table 3-3. Example 1--Potential to Emit Calculation for an Elevated Release from the Western Portion of the 300 Area

\begin{tabular}{|c|c|c|c|c|c|}
\hline Nuclide & $\begin{array}{c}\text { Unabated } \\
\text { annual } \\
\text { releases } \\
(\mathrm{Ci} / \mathrm{yr})\end{array}$ & $\begin{array}{c}\text { Adjustment } \\
\text { factor } \\
\text { for HEPA } \\
\text { filters }\end{array}$ & $\begin{array}{c}\text { Abated } \\
\text { annual } \\
\text { release } \\
(\mathrm{Ci} / \mathrm{yr})\end{array}$ & $\begin{array}{c}300 \text { West } \\
\text { Area unit } \\
\text { dose factors } \\
\text { (mrem/Ci) }\end{array}$ & $\begin{array}{c}\text { Unabated and abated } \\
\text { potential to emit to } \\
\text { NOR } \\
\text { (mrem/y) }\end{array}$ \\
\hline $\mathrm{H}-3$ & $1.1 \mathrm{E}+01$ & 1 & $1.1 \mathrm{E}+01$ & $1.2 \mathrm{E}-04$ & $1.3 \mathrm{E}-03$ \\
\hline $\mathrm{Co}-60$ & $2.7 \mathrm{E}-03$ & 1 & $2.7 \mathrm{E}-03$ & $1.6 \mathrm{E}+00$ & $4.3 \mathrm{E}-03$ \\
\hline $\mathrm{Se}-79$ & $1.5 \mathrm{E}-04$ & 1 & $1.5 \mathrm{E}-04$ & $7.9 \mathrm{E}-01$ & $1.2 \mathrm{E}-04$ \\
\hline $\mathrm{Sr}-90$ & $2.4 \mathrm{E}-04$ & 1 & $2.4 \mathrm{E}-04$ & $7.1 \mathrm{E}-01$ & $1.7 \mathrm{E}-04$ \\
\hline $\mathrm{Y}-90$ & $2.4 \mathrm{E}-04$ & 1 & $2.4 \mathrm{E}-04$ & $2.2 \mathrm{E}-03$ & $5.3 \mathrm{E}-07$ \\
\hline $\mathrm{Zr}-95$ & 0.0 & 1 & 0.0 & $2.5 \mathrm{E}-02$ & 0.0 \\
\hline $\mathrm{Cs}-137$ & $4.0 \mathrm{E}-03$ & 1 & $4.0 \mathrm{E}-03$ & $1.7 \mathrm{E}-01$ & $6.8 \mathrm{E}-04$ \\
\hline $\mathrm{Ba}-137 \mathrm{~m}$ & $3.8 \mathrm{E}-03$ & 1 & $3.8 \mathrm{E}-03$ & $1.4 \mathrm{E}-05$ & $5.3 \mathrm{E}-08$ \\
\hline $\mathrm{Pu}-239$ & $9.0 \mathrm{E}-06$ & 1 & $9.0 \mathrm{E}-06$ & $5.0 \mathrm{E}+01$ & $4.5 \mathrm{E}-04$ \\
\hline $\mathrm{Pu}-241$ & $5.3 \mathrm{E}-04$ & 1 & $5.3 \mathrm{E}-04$ & $7.9 \mathrm{E}-01$ & $4.2 \mathrm{E}-04$ \\
\hline $\mathrm{Am}-241$ & $1.1 \mathrm{E}-05$ & 1 & $1.1 \mathrm{E}-05$ & $7.7 \mathrm{E}+01$ & $1.1 \mathrm{E}-03$ \\
\hline & & & & Total Dose: & $8.3 \mathrm{E}-03$ \\
\hline
\end{tabular}

The adjustment factor is 1 rather than 0.01 , because the source term is heated to $\geq 100^{\circ} \mathrm{C}$ and the temperature of the emissions is $\geq 100^{\circ} \mathrm{C}$.

\section{EXAMPLE 2:}

The potential to emit is calculated using Method 1 for a contaminated burial ground located in the 300 Area and within 1,000 meters from the Columbia River. All releases occur at ground level with no abatement technology being used. The burial ground is a 1,000 meters by 500 meters area (5.0 E+05 square meters) and is located 1,200 meters from the NOR. The radioactive source term within the burial ground is contaminated soil, which recently was determined from the analysis of soil samples.

Using 40 CFR 61 , Appendix D, the release factor for this inventory is $1.0 \mathrm{E}-03$, because the source term is a particulate solid. The effective diameter of the burial ground is 798 meters, using Equation 3.10. The distance/diameter ratio is 1.50 , which is well above the 0.22 minimum recommended in Section 3.4.6.

Table 3-4 provides the potential to emit data and subsequent doses to the NOR for Example 2 . 
Table 3-4. Example 2--Potential to Emit Calculation for a Ground Level Release from the Eastern Portion of the 300 Area.

\begin{tabular}{|c|c|c|c|c|c|}
\hline Nuclide & $\begin{array}{c}\text { Inventory } \\
\text { in soil } \\
(\mathrm{Ci})\end{array}$ & $\begin{array}{c}\text { Appendix D } \\
\text { release } \\
\text { factor }\end{array}$ & $\begin{array}{c}\text { Unabated and abated } \\
\text { release } \\
\text { rate } \\
(\mathrm{Ci} / \mathrm{yr})\end{array}$ & $\begin{array}{c}300 \text { East } \\
\text { Area unit } \\
\text { dose factors } \\
(\mathrm{mrem} / \mathrm{Ci})\end{array}$ & $\begin{array}{c}\text { Unabated and abated } \\
\text { PTE to NOR } \\
(\mathrm{mrem} / \mathrm{y})\end{array}$ \\
\hline $\mathrm{U}-234$ & $2.5 \mathrm{E}+00$ & $1 \mathrm{E}-03$ & $2.5 \mathrm{E}-03$ & $7.4 \mathrm{E}+01$ & $1.9 \mathrm{E}-01$ \\
\hline $\mathrm{U}-235$ & $1.2 \mathrm{E}-01$ & $1 \mathrm{E}-03$ & $1.2 \mathrm{E}-04$ & $7.1 \mathrm{E}+01$ & $8.5 \mathrm{E}-03$ \\
\hline $\mathrm{U}-238$ & $2.5 \mathrm{E}+00$ & $1 \mathrm{E}-03$ & $2.5 \mathrm{E}-03$ & $6.6 \mathrm{E}+01$ & $1.7 \mathrm{E}-01$ \\
\hline \multicolumn{7}{|r}{} & & Total Dose: & $3.6 \mathrm{E}-01$ \\
\hline
\end{tabular}

a The adjustment factor is 1 rather than 0.01 , because the source term is heated to $\geq 100^{\circ} \mathrm{C}$ and the temperature of the emissions is $\geq 100^{\circ} \mathrm{C}$.

\subsection{REPORTING RELEASES AND DOSES IN A NOTICE OF CONSTRUCTION}

Once the potential to emit is determined for a NOC, it is critical that the releases and doses be put into terms that are consistent with the measurement method. The potential to emit values generally become the emission limits for an NOC, making this conversion essential for demonstrating compliance to the emission limits.

If the NOC only addresses one activity associated with an existing emission unit, it is also critical to make sure the emission limits are established for the emission unit rather than the activity. One would need to add the releases and doses associated with the activity to the releases and doses of the emission unit. It is usually very difficult or impossible to demonstrate compliance to a NOC when the emission limits are for a specific activity and the compliance measurement is for the entire emission unit and all the activities ventilated by emission unit.

The emission limits should be checked for detectability before specifying the limits and measurement method in the NOC. Measurements that are not sensitive enough to verify that the emission limits have not been exceeded are useless. The method for determining whether or not a record sampling method is sensitive enough to verify the emission limits of a stack is outlined in Equation 3.11. Equation 3.11 only applies to emission units using record sampling. Raising the emission limits, reducing the flow rate of a stack, limiting the time that the stack operates, and/or lengthening the sample period are all methods that can make a record sampling method more detectable. Similar methods for determining detectability will need to be used for other measurement methods.

Equation 3.11: $\quad \mathrm{MDL}=0.02832(\mathrm{MDC})\left(\mathrm{V}_{\text {stk }}\right)$

MDL - minimum detection level for an analyte on a specific stack, $\mathrm{Ci} / \mathrm{yr}$.

MDC - minimum detectable concentration for an analyte, $\mu \mathrm{Ci} / \mathrm{mL}$.

$\mathrm{V}_{\text {stk }} \quad$ - annual stack discharge volume, $\mathrm{ft}^{3} / \mathrm{yr}$. 
Equation 3.12:

$$
\mathrm{MDC}=3.531 \mathrm{E}-11\left(\frac{\mathrm{MDA}}{\mathrm{V}_{\mathrm{s}}}\right)
$$

MDC - minimum detectable concentration for an analyte, $\mu \mathrm{Ci} / \mathrm{mL}$.

MDA - minimum detectable activity for an analyte, $\mathrm{pCi}$.

$\mathrm{V}_{\mathrm{s}} \quad$ - sample volume, $\mathrm{ft}^{3}$.

When measurement methods other than record sampling are used, it is essential that the NOC specify how the measurement will be used to demonstrate compliance with the emission limits specified in a NOC. As an example, the NOC for portable temporary radioactive air emission units (PTREAUs) uses handling limits to link the measurements to the emission limits (DOE/RL-96-75). The PTRAEU NOC uses contamination smear data to verify the handling limits specified in the NOC. The handling limits were established such that the emission limits could not be exceeded unless the handling limits were exceeded.

Example 3 is being used to illustrate why these considerations are significant.

\section{EXAMPLE 3:}

A hypothetical facility located in the 200 East Area contains contaminated equipment. The main building of the facility is ventilated by a single stack that is equipped with a single HEPA filter and operates continuously at 2,500 cubic feet per minute ( 1.18 cubic meters per second). The stack is a nondesignated (i.e., minor) emission unit and the periodic confirmatory measurement method is record sampling. The facility is planning to sample the stack continuously and exchange the record samples biweekly (i.e., every 2 weeks). Only four of the expected 26 record samples will be analyzed and used to demonstrate compliance. Each of the samples used for the periodic confirmatory measurements will analyzed for total alpha and beta activity. The stack has been assessed with the following potential to emit, provided in Table 3-5.

Table 3-5. Example 3--Original Assessed Potential to Emit for the Stack.

\begin{tabular}{|c|c|c|c|c|c|c|}
\hline Nuclide & $\begin{array}{c}\text { Unabated } \\
\text { release } \\
\text { rate } \\
(\mathrm{Ci} / \mathrm{yr})\end{array}$ & $\begin{array}{c}\text { HEPA filter } \\
\text { factor }\end{array}$ & $\begin{array}{c}\text { Abated } \\
\text { release } \\
\text { rate } \\
(\mathrm{Ci} / \mathrm{yr})\end{array}$ & $\begin{array}{c}\text { 200 East } \\
\text { Area unit } \\
\text { dose factors } \\
(\mathrm{mrem} / \mathrm{Ci})\end{array}$ & $\begin{array}{c}\text { Unabated } \\
\text { dose to NOR } \\
\text { (mrem/yr) }\end{array}$ & $\begin{array}{c}\text { Abated } \\
\text { dose to NOR } \\
\text { (mrem/yr) }\end{array}$ \\
\hline $\mathrm{Sr}-90$ & $2.2 \mathrm{E}-05$ & 3,000 & $7.3 \mathrm{E}-09$ & $1.1 \mathrm{E}-01$ & $2.4 \mathrm{E}-06$ & $8.1 \mathrm{E}-10$ \\
\hline $\mathrm{Y}-90$ & $2.2 \mathrm{E}-05$ & 3,000 & $7.3 \mathrm{E}-09$ & $3.4 \mathrm{E}-04$ & $7.5 \mathrm{E}-09$ & $2.5 \mathrm{E}-12$ \\
\hline $\mathrm{Cs}-137$ & $5.3 \mathrm{E}-05$ & 3,000 & $1.8 \mathrm{E}-08$ & $2.7 \mathrm{E}-02$ & $1.4 \mathrm{E}-06$ & $4.8 \mathrm{E}-10$ \\
\hline $\mathrm{Ba}-137 \mathrm{~m}$ & $5.0 \mathrm{E}-05$ & 3,000 & $1.7 \mathrm{E}-08$ & $5.3 \mathrm{E}-13$ & $2.7 \mathrm{E}-17$ & $8.9 \mathrm{E}-21$ \\
\hline $\mathrm{Pu}-239$ & $1.6 \mathrm{E}-06$ & 3,000 & $5.3 \mathrm{E}-10$ & $8.2 \mathrm{E}+00$ & $1.3 \mathrm{E}-05$ & $4.4 \mathrm{E}-09$ \\
\hline $\mathrm{Pu}-240$ & $1.6 \mathrm{E}-06$ & 3,000 & $5.3 \mathrm{E}-10$ & $8.2 \mathrm{E}+00$ & $1.3 \mathrm{E}-05$ & $4.4 \mathrm{E}-09$ \\
\hline $\mathrm{Pu}-241$ & $3.0 \mathrm{E}-04$ & 3,000 & $1.0 \mathrm{E}-07$ & $1.3 \mathrm{E}-01$ & $3.9 \mathrm{E}-05$ & $1.3 \mathrm{E}-08$ \\
\hline $\mathrm{Am}-241$ & $1.5 \mathrm{E}-04$ & 3,000 & $5.8 \mathrm{E}-08$ & $1.3 \mathrm{E}+01$ & $2.0 \mathrm{E}-03$ & $6.5 \mathrm{E}-07$ \\
\hline & & & Total Dose: & $2.0 \mathrm{E}-03$ & $6.7 \mathrm{E}-07$ \\
\hline
\end{tabular}


The measurement method only includes total alpha and total beta activity analyses. It is essential that the nuclide specific information in Table 3-5 be put into terms of total alpha activity and total beta activity. Table 3-6 contains the converted data. The unabated releases for all of the alpha emitting nuclides were summed for Table 3-6. The unabated releases for all of the beta emitting nuclides were also summed for Table 3-6. The most conservative unit dose factors for the alpha and beta emitting nuclides were used to calculate the doses. Note that $\mathrm{Ba}-137 \mathrm{~m}$ was omitted from the table because it only emits gamma rays and its unabated potential to emit is not $>10$ percent of the total potential to emit for the stack, greater than 0.1 millirem per year, or greater than 25 percent of the abated potential to emit.

Table 3-6. Example 3--Original Assessed Potential to Emit for the Stack's NOC.

\begin{tabular}{|c|c|c|c|c|c|c|}
\hline Nuclide & $\begin{array}{c}\text { Unabated } \\
\text { release } \\
\text { rate } \\
(\mathrm{Ci} / \mathrm{yr})\end{array}$ & $\begin{array}{c}\text { HEPA filter } \\
\text { factor }\end{array}$ & $\begin{array}{c}\text { Abated } \\
\text { release } \\
\text { rate } \\
(\mathrm{Ci} / \mathrm{yr})\end{array}$ & $\begin{array}{c}200 \mathrm{East} \\
\text { Area unit } \\
\text { dose factors } \\
(\mathrm{mrem} / \mathrm{Ci})\end{array}$ & $\begin{array}{c}\text { Unabated } \\
\text { dose to NOR } \\
\text { (mrem/yr) }\end{array}$ & $\begin{array}{c}\text { Abated } \\
\text { dose to NOR } \\
\text { (mrem/yr) }\end{array}$ \\
\hline Total alpha & $1.5 \mathrm{E}-04$ & 3,000 & $5.1 \mathrm{E}-08$ & $1.3 \mathrm{E}+01$ & $2.0 \mathrm{E}-03$ & $6.6 \mathrm{E}-07$ \\
\hline Total beta & $4.0 \mathrm{E}-04$ & 3,000 & $1.3 \mathrm{E}-07$ & $1.3 \mathrm{E}-01$ & $5.2 \mathrm{E}-05$ & $1.7 \mathrm{E}-08$ \\
\hline \multicolumn{7}{|r}{} \\
\hline
\end{tabular}

A new activity is proposed for the facility. The facility is needed to repackage some material over the course of several months. The potential to emit releases and doses associated with the specific activity has been assessed and is included as Table 3-7.

Table 3-7. Example 3--Assessed Potential to Emit for the New Activity.

\begin{tabular}{|c|c|c|c|c|c|c|}
\hline Nuclide & $\begin{array}{c}\text { Unabated } \\
\text { release } \\
\text { rate } \\
(\mathrm{Ci} / \mathrm{yr})\end{array}$ & $\begin{array}{c}\text { HEPA filter } \\
\text { factor }\end{array}$ & $\begin{array}{c}\text { Abated } \\
\text { release } \\
\text { rate } \\
(\mathrm{Ci} / \mathrm{yr})\end{array}$ & $\begin{array}{c}\text { 200 East } \\
\text { Area unit } \\
\text { dose factors } \\
(\mathrm{mrem} / \mathrm{C})\end{array}$ & $\begin{array}{c}\text { Unabated } \\
\text { dose to NOR } \\
(\mathrm{mrem} / \mathrm{yr})\end{array}$ & $\begin{array}{c}\text { Abated } \\
\text { dose to NOR } \\
\text { (mrem/yr) }\end{array}$ \\
\hline $\mathrm{H}-3$ & $1.0 \mathrm{E}+00$ & 1 & $1.0 \mathrm{E}+00$ & $2.5 \mathrm{E}-05$ & $2.5 \mathrm{E}-05$ & $2.5 \mathrm{E}-05$ \\
\hline $\mathrm{Cs}-137$ & $4.3 \mathrm{E}-04$ & 3,000 & $1.4 \mathrm{E}-07$ & $2.7 \mathrm{E}-02$ & $1.2 \mathrm{E}-05$ & $3.9 \mathrm{E}-09$ \\
\hline $\mathrm{Ba}-137 \mathrm{~m}$ & $4.1 \mathrm{E}-04$ & 3,000 & $1.4 \mathrm{E}-07$ & $5.3 \mathrm{E}-13$ & $2.2 \mathrm{E}-16$ & $7.2 \mathrm{E}-20$ \\
\hline $\mathrm{Eu}-152$ & $6.2 \mathrm{E}-05$ & 3,000 & $2.1 \mathrm{E}-09$ & $2.4 \mathrm{E}-01$ & $1.5 \mathrm{E}-06$ & $5.0 \mathrm{E}-10$ \\
\hline & & & Total Dose: & $3.8 \mathrm{E}-05$ & $2.5 \mathrm{E}-05$ \\
\hline
\end{tabular}

The potential to emit for this activity needs to be added into the potential to emit for the stack to determine the designation of the stack and to establish the emission limits for the stack. Table 3-8 provides the assessed potential to emit for all activities ventilated by this stack. 
Table 3-8. Example 3--Assessed Potential to Emit for the Stack, Including New Activity.

\begin{tabular}{|c|c|c|c|c|c|c|}
\hline Nuclide & $\begin{array}{c}\text { Unabated } \\
\text { release } \\
\text { rate } \\
(\mathrm{Ci} / \mathrm{yr})\end{array}$ & $\begin{array}{c}\text { HEPA filter } \\
\text { factor }\end{array}$ & $\begin{array}{c}\text { Abated } \\
\text { release } \\
\text { rate } \\
(\mathrm{Ci} / \mathrm{yr})\end{array}$ & $\begin{array}{c}200 \mathrm{East} \\
\text { Area Unit } \\
\text { Dose Factors } \\
(\mathrm{mrem} / \mathrm{Ci})\end{array}$ & $\begin{array}{c}\text { Unabated } \\
\text { dose to NOR } \\
(\mathrm{mrem} / \mathrm{yr})\end{array}$ & $\begin{array}{c}\text { Abated } \\
\text { dose to NOR } \\
\text { (mrem/yr) }\end{array}$ \\
\hline $\mathrm{H}-3$ & $1.0 \mathrm{E}+00$ & 1 & $1.0 \mathrm{E}+00$ & $2.5 \mathrm{E}-05$ & $2.5 \mathrm{E}-05$ & $2.5 \mathrm{E}-05$ \\
\hline $\mathrm{Sr}-90$ & $2.2 \mathrm{E}-05$ & 3,000 & $7.3 \mathrm{E}-09$ & $1.1 \mathrm{E}-01$ & $2.4 \mathrm{E}-06$ & $8.1 \mathrm{E}-10$ \\
\hline $\mathrm{Y}-90$ & $2.2 \mathrm{E}-05$ & 3,000 & $7.3 \mathrm{E}-09$ & $3.4 \mathrm{E}-04$ & $7.5 \mathrm{E}-09$ & $2.5 \mathrm{E}-12$ \\
\hline $\mathrm{Cs}-137$ & $4.8 \mathrm{E}-04$ & 3,000 & $1.6 \mathrm{E}-07$ & $2.7 \mathrm{E}-02$ & $1.3 \mathrm{E}-05$ & $4.3 \mathrm{E}-09$ \\
\hline $\mathrm{Ba}-137 \mathrm{~m}$ & $4.6 \mathrm{E}-04$ & 3,000 & $1.5 \mathrm{E}-07$ & $5.3 \mathrm{E}-13$ & $2.4 \mathrm{E}-16$ & $8.1 \mathrm{E}-20$ \\
\hline $\mathrm{Eu}-152$ & $6.2 \mathrm{E}-06$ & 3,000 & $2.1 \mathrm{E}-09$ & $2.4 \mathrm{E}-01$ & $1.5 \mathrm{E}-06$ & $5.0 \mathrm{E}-10$ \\
\hline $\mathrm{Pu}-239$ & $1.6 \mathrm{E}-06$ & 3,000 & $5.3 \mathrm{E}-10$ & $8.2 \mathrm{E}+00$ & $1.3 \mathrm{E}-05$ & $4.4 \mathrm{E}-09$ \\
\hline $\mathrm{Pu}-240$ & $1.6 \mathrm{E}-06$ & 3,000 & $5.3 \mathrm{E}-10$ & $8.2 \mathrm{E}+00$ & $1.3 \mathrm{E}-05$ & $4.4 \mathrm{E}-09$ \\
\hline $\mathrm{Pu}-241$ & $3.0 \mathrm{E}-04$ & 3,000 & $1.0 \mathrm{E}-07$ & $1.3 \mathrm{E}-01$ & $3.9 \mathrm{E}-05$ & $1.3 \mathrm{E}-08$ \\
\hline $\mathrm{Am}-241$ & $1.5 \mathrm{E}-04$ & 3,000 & $5.8 \mathrm{E}-08$ & $1.3 \mathrm{E}+01$ & $2.0 \mathrm{E}-03$ & $6.5 \mathrm{E}-07$ \\
\hline & & & & Total Dose: & $2.0 \mathrm{E}-03$ & $6.8 \mathrm{E}-07$ \\
\hline
\end{tabular}

Again, the measurement method only includes total alpha and total beta activity analyses. It is essential that the nuclide specific information in Table 3-8 be put into terms of total alpha activity and total beta activity. Table 3-9 contains the converted data. Note that $\mathrm{H}-3$, a beta emitting nuclide, was not included in the conversion to total beta releases. $\mathrm{H}-3$ is a vapor that is not sampled by the measurement method. $\mathrm{H}-3$ does not need to be sampled for, because its unabated potential to emit is not $>10$ percent of the total potential to emit for the stack, greater than 0.1 millirem per year, or greater than 25 percent of the abated potential to emit. Also, Eu-152 only decays by beta emission 27.8 percent of the time and its activity was adjusted by a factor of 0.278 when calculating the total beta releases. As before, Ba- $137 \mathrm{~m}$ was omitted from the table because it only emits gamma rays and it did not require sampling.

Table 3-9. Example 3--Assessed Potential to Emit for the Stack Notice of Construction.

\begin{tabular}{|c|c|c|c|c|c|c|}
\hline Nuclide & $\begin{array}{c}\text { Unabated } \\
\text { release } \\
\text { rate } \\
(\mathrm{Ci} / \mathrm{yr}) \\
\end{array}$ & $\begin{array}{l}\text { HEPA filter } \\
\text { factor }\end{array}$ & $\begin{array}{c}\text { Abated } \\
\text { release } \\
\text { rate } \\
(\mathrm{Ci} / \mathrm{yr}) \\
\end{array}$ & $\begin{array}{c}200 \text { East } \\
\text { Area unit } \\
\text { dose factors } \\
(\mathrm{mrem} / \mathrm{Ci})\end{array}$ & $\begin{array}{l}\text { Unabated } \\
\text { dose to NOR } \\
\text { (mrem/yr) }\end{array}$ & $\begin{array}{c}\text { Abated } \\
\text { dose to NOR } \\
\text { (mrem/y) }\end{array}$ \\
\hline Total alpha & $1.5 \mathrm{E}-04$ & 3,000 & $5.1 \mathrm{E}-08$ & $1.3 \mathrm{E}+01$ & $2.0 \mathrm{E}-03$ & $6.6 \mathrm{E}-07$ \\
\hline Total beta & 8.3 E-04 & 3,000 & $2.8 \mathrm{E}-07$ & $2.4 \mathrm{E}-01$ & $2.0 \mathrm{E}-04$ & $6.6 \mathrm{E}-08$ \\
\hline \multicolumn{5}{|r|}{ Total Dose: } & $2.2 \mathrm{E}-03$ & $7.3 \mathrm{E}-07$ \\
\hline
\end{tabular}

The final step is to check the detectability of the measurement method. A two-week sample typically has a sample volume of 40,000 cubic feet (1,133 cubic meters). The minimum detectable concentrations for a two week sample are $1.0 \mathrm{E}-15 \mu \mathrm{Ci} / \mathrm{mL}$ for total alpha activity and $9.5 \mathrm{E}-15 \mu \mathrm{Ci} / \mathrm{mL}$ for total beta activity. Remember the stack operates continuously at 2,500 cubic feet per minute $(1.18$ cubic meters per second). The unit dose factors for Am-241 and Eu-152 are used to calculate the total alpha and total 
beta doses, respectively, because they were the most conservative unit dose factors of all the nuclides included in the assessment. Table 3-10 displays the data and the results of the detectability calculations, when the sampling frequency is biweekly and the stack operates at 2,500 cubic feet per minute (1.18 cubic meters per second).

Table 3-10. Example 3--Detectability Check for Biweekly Sampling when the Stack Operates at 2,500 Cubic Feet per Minute.

\begin{tabular}{|c|c|c|c|c|c|c|c|}
\hline Nuclide & $\begin{array}{c}\text { Average } \\
\text { stack } \\
\text { flow rate } \\
(\mathrm{cfm})\end{array}$ & $\begin{array}{c}\text { Annual } \\
\text { operating } \\
\text { time } \\
(\mathrm{hr})\end{array}$ & $\begin{array}{c}\text { Analysis } \\
\text { minimum } \\
\text { detectable } \\
\text { concentration } \\
(\mu \mathrm{Ci} / \mathrm{mL})\end{array}$ & $\begin{array}{c}\text { Unit dose } \\
\text { factor } \\
(\mathrm{mrem} / \mathrm{Ci})\end{array}$ & $\begin{array}{c}\text { Annual } \\
\text { discharge } \\
\text { volume } \\
(\mathrm{mL})\end{array}$ & $\begin{array}{c}\text { Maximum } \\
\text { detection } \\
\text { limit } \\
\text { Activity } \\
(\mathrm{Ci} / \mathrm{yr})\end{array}$ & $\begin{array}{c}\text { Maximum } \\
\text { detection limit } \\
\text { dose } \\
(\mathrm{mrem} / \mathrm{yr})\end{array}$ \\
\hline Alpha & 2,500 & 8,760 & $1.0 \mathrm{E}-15$ & $1.3 \mathrm{E}+01$ & $3.7 \mathrm{E}+13$ & $3.7 \mathrm{E}-08$ & $4.8 \mathrm{E}-07$ \\
\hline Beta & 2,500 & 8,760 & $9.5 \mathrm{E}-15$ & $2.4 \mathrm{E}-01$ & $3.7 \mathrm{E}+13$ & $3.5 \mathrm{E}-07$ & $8.5 \mathrm{E}-08$ \\
\hline
\end{tabular}

Note that the measurement method is not sensitive enough for detecting the emission limits of total beta activity. As a result, the measurement method needs to be modified or the flow rate for the stack can be lowered. The easiest modification would be to lengthen the sampling period to 4 weeks (i.e., monthly sampling). This would double the sample volume and lower the minimum detectable concentrations by 50 percent. Table 3-11 displays the results using a monthly sampling frequency.

Table 3-11. Example 3--Detectability Check for Monthly Sampling when the Stack Operates at 2,500 Cubic Feet per Minute.

\begin{tabular}{|c|c|c|c|c|c|c|c|}
\hline Nuclide & $\begin{array}{c}\text { Average } \\
\text { stack } \\
\text { flow rate } \\
(\mathrm{cfm})\end{array}$ & $\begin{array}{c}\text { Annual } \\
\text { operating } \\
\text { time } \\
(\mathrm{hr})\end{array}$ & $\begin{array}{c}\text { Analysis } \\
\text { minimum } \\
\text { detectable } \\
\text { concentration } \\
(\mu \mathrm{Ci} / \mathrm{mL})\end{array}$ & $\begin{array}{c}\text { Unit dose } \\
\text { factor } \\
(\mathrm{mrem} / \mathrm{Ci})\end{array}$ & $\begin{array}{c}\text { Annual } \\
\text { discharge } \\
\text { volume } \\
(\mathrm{mL})\end{array}$ & $\begin{array}{c}\text { Maximum } \\
\text { detection } \\
\text { imit } \\
\text { activity } \\
(\mathrm{Ci} / \mathrm{yr})\end{array}$ & $\begin{array}{c}\text { Maximum } \\
\text { detection limit } \\
\text { dose } \\
(\mathrm{mrem} / \mathrm{yr})\end{array}$ \\
\hline Alpha & 2,500 & 8,760 & $5.0 \mathrm{E}-16$ & $1.3 \mathrm{E}+01$ & $3.7 \mathrm{E}+13$ & $1.9 \mathrm{E}-08$ & $2.4 \mathrm{E}-07$ \\
\hline Beta & 2,500 & 8,760 & $4.8 \mathrm{E}-15$ & $2.4 \mathrm{E}-01$ & $3.7 \mathrm{E}+13$ & $1.8 \mathrm{E}-07$ & $4.3 \mathrm{E}-08$ \\
\hline
\end{tabular}

Using a monthly sampling frequency ensures that the emission limits for both total alpha and total beta activity are detectable. Another alternative to improving the detectability would have been to lower the stack flow rate. Table 3-12 display the results for the scenario where lowering the stack flow rate by 600 cubic feet per minute ( 0.283 cubic meters per second) was chosen over lengthening the sampling period. Note that a similar effect can be obtained by not operating the stack for planned time intervals. 
HNF-3602, Vol. 1

Table 3-12. Example 3--Detectability Check for Biweekly Sampling when the Stack Operates at 1,900 Cubic Feet per Minute.

\begin{tabular}{|c|c|c|c|c|c|c|c|}
\hline Nuclide & $\begin{array}{c}\text { Average } \\
\text { stack } \\
\text { flow rate } \\
(\mathrm{cfm})\end{array}$ & $\begin{array}{c}\text { Annual } \\
\text { operating } \\
\text { time } \\
(\mathrm{hr})\end{array}$ & $\begin{array}{c}\text { Analysis } \\
\text { minimum } \\
\text { detectable } \\
\text { concentration } \\
(\mu \mathrm{Ci} / \mathrm{mL})\end{array}$ & $\begin{array}{c}\text { Unit dose } \\
\text { factor } \\
(\mathrm{mrem} / \mathrm{Ci})\end{array}$ & $\begin{array}{c}\text { Annual } \\
\text { discharge } \\
\text { volume } \\
(\mathrm{mL})\end{array}$ & $\begin{array}{c}\text { Maximum } \\
\text { detection } \\
\text { limit } \\
\text { activity } \\
(\mathrm{Ci} / \mathrm{yr})\end{array}$ & $\begin{array}{c}\text { Maximum } \\
\text { detection limit } \\
\text { dose } \\
(\mathrm{mrem} / \mathrm{yr})\end{array}$ \\
\hline Alpha & 1,900 & 8,760 & $1.0 \mathrm{E}-15$ & $1.3 \mathrm{E}+01$ & $2.8 \mathrm{E}+13$ & $2.8 \mathrm{E}-08$ & $3.7 \mathrm{E}-07$ \\
\hline Beta & 1,900 & 8,760 & $9.5 \mathrm{E}-15$ & $2.4 \mathrm{E}-01$ & $2.8 \mathrm{E}+13$ & $2.7 \mathrm{E}-07$ & $6.4 \mathrm{E}-08$ \\
\hline
\end{tabular}


HNF-3602, Vol. 1

This page intentionally left blank. 


\subsection{UNIT DOSE FACTORS FOR AIR EMISSIONS}

The tables in this section contain unit dose factors for each emission zone and at two release heights. Dose factors have been provided for each of the 265 radionuclides in the CAP88-PC library. The radionuclides in the tables have been sorted by atomic number and by atomic weight.

Figure 4-1 displays each of the emission zones on the Hanford Site. The 300 Area has been subdivided into two emission zones ( 300 East and 300 West), because dispersion factors were significantly different at various locations within the 300 Area. The dispersion factors varied significantly because of the close proximity of the 300 Area to the Hanford Site boundary. The dividing line between these two zones is marked by George Washington Way and the western boundary for the 300-FF-1 Operable Unit. This boundary also can be described as being approximately $318 \pm 50$ meters from the western edge of the Columbia River.

The release locations and the meteorological stations that were used in calculating the unit dose factors for each emission zone are listed in Table 4-1. The distance and direction to the NOR for each emission zone are provided in Table 4-2.

Tables 4-3 through 4-13 contain the unit dose factors for each of the 11 emission zones. The technical bases for the unit dose factors provided in this section can be found in Volume 2 of this document. 


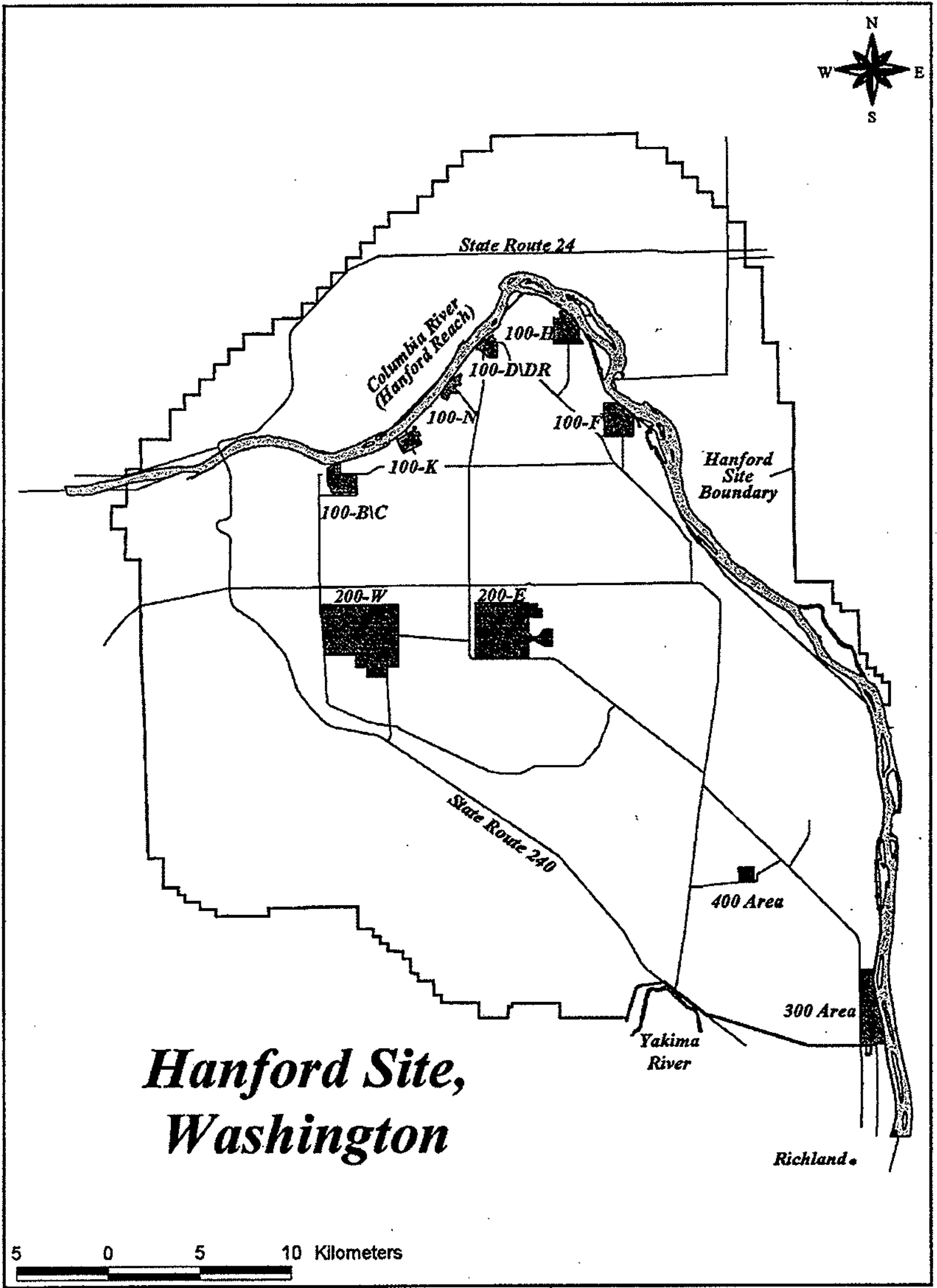

Figure 4-1. Hanford Site Map of Emission Zones. 
HNF-3602, Vol. 1

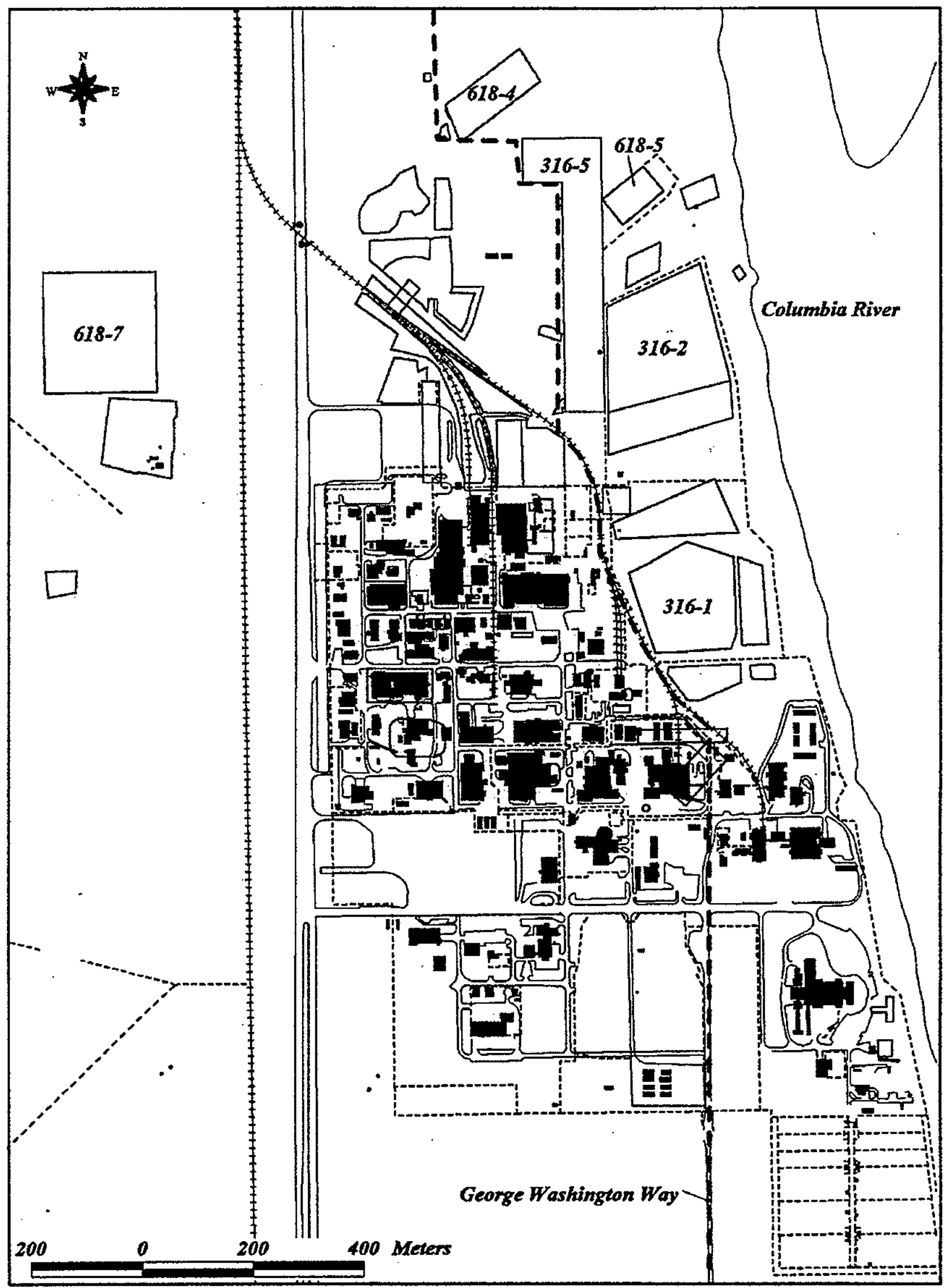

Figure 4-2. 300 Area Map of Emission Zones. 
Table 4-1. Release Locations and Meteorological Stations Used.

\begin{tabular}{|c|c|c|c|}
\hline \multicolumn{3}{|c|}{ Release Locations $^{a}$} & \multirow{2}{*}{$\begin{array}{l}\text { Meteorological } \\
\text { station }^{\mathrm{b}}\end{array}$} \\
\hline $\begin{array}{l}\text { Emission zone } \\
\text { (building/facility) }\end{array}$ & $\begin{array}{l}\text { Geographic } \\
\text { coordinates }\end{array}$ & $\begin{array}{l}\text { Washington State } \\
\text { plane coordinates }\end{array}$ & \\
\hline $\begin{array}{c}100-\mathrm{B} / \mathrm{C} \\
(105-\mathrm{B} \text { Bldg.) }\end{array}$ & $\begin{array}{l}46^{\circ} 37^{\prime} 49.2^{\prime \prime} \text { long. } \\
119^{\circ} 38^{\prime} 51.1^{\prime \prime} \text { lat. }\end{array}$ & $\begin{array}{l}565,275 \mathrm{~m} \mathrm{E} \\
144,515 \mathrm{~m} \mathrm{~N}\end{array}$ & $\begin{array}{c}\text { GABW (\#23) } \\
1986-1996\end{array}$ \\
\hline $\begin{array}{c}100-\mathrm{D} / \mathrm{DR} \\
(105-\mathrm{D} \text { Bldg. })\end{array}$ & $\begin{array}{l}46^{\circ} 41^{\prime} 35.2^{n} \text { long. } \\
119^{\circ} 32^{*} 8.4^{\prime \prime} \text { lat. }\end{array}$ & $\begin{array}{l}573,755 \mathrm{~m} \mathrm{E} \\
151,590 \mathrm{~m} \mathrm{~N}\end{array}$ & $\begin{array}{l}100 \mathrm{~N}(\# 13) \\
1983-1996\end{array}$ \\
\hline $\begin{array}{c}100-F \\
\text { (105-F Bldg.) }\end{array}$ & $\begin{array}{l}46^{\circ} 39^{\prime} 23.2^{\prime \prime} \text { long. } \\
119^{\circ} 26^{\prime} 56.2^{\prime \prime} \text { lat. }\end{array}$ & $\begin{array}{l}580,440 \mathrm{~m} \mathrm{E} \\
147,600 \mathrm{~m} \mathrm{~N}\end{array}$ & $\begin{array}{l}100 F(\# 24) \\
1986-1996\end{array}$ \\
\hline $\begin{array}{c}100-\mathrm{H} \\
(105-\mathrm{H} \text { Bldg. }\end{array}$ & $\begin{array}{l}46^{\circ} 42^{\prime} 4.0^{\prime \prime} \text { long. } \\
119^{\circ} 28^{\prime} 58.4^{n} \text { lat. }\end{array}$ & $\begin{array}{l}577,780 \mathrm{~m} \mathrm{E} \\
152,530 \mathrm{~m} \mathrm{~N}\end{array}$ & $\begin{array}{l}100 \mathrm{~F}(\# 24) \\
1986-1996\end{array}$ \\
\hline $\begin{array}{c}100-\mathrm{K} \\
(105-\mathrm{KW} \text { Bldg. })\end{array}$ & $\begin{array}{l}46^{\circ} 38^{\prime} 50.4^{\prime \prime} \text { long. } \\
119^{\circ} 36^{\prime} 11.9^{\prime \prime} \text { lat. }\end{array}$ & $\begin{array}{l}568,640 \mathrm{~m} \mathrm{E} \\
146,440 \mathrm{~m} \mathrm{~N}\end{array}$ & $\begin{array}{c}\text { GABW (\#23) } \\
1986-1996\end{array}$ \\
\hline $\begin{array}{c}100-N \\
(105-N \text { Bldg })\end{array}$ & $\begin{array}{l}46^{\circ} 40^{\prime} 29.0^{\prime \prime} \text { long. } \\
119^{\circ} 34^{\prime} 8.8^{\prime \prime} \text { lat. }\end{array}$ & $\begin{array}{l}571,220 \mathrm{~m} \mathrm{E} \\
149,515 \mathrm{~m} \mathrm{~N}\end{array}$ & $\begin{array}{l}100 N(\# 13) \\
1983-1996\end{array}$ \\
\hline $\begin{array}{c}200-W \\
\text { (REDOX) }\end{array}$ & $\begin{array}{l}46^{\circ} 32^{\prime} 5.5^{\prime \prime} \text { long. } \\
119^{\circ} 37^{\prime} 16.7^{\prime \prime} \text { lat. }\end{array}$ & $\begin{array}{l}567,400 \mathrm{~m} \mathrm{E} \\
133,925 \mathrm{~m} \mathrm{~N}\end{array}$ & $\begin{array}{l}200 W(\# 7) \\
1983-1996\end{array}$ \\
\hline $\begin{array}{c}\text { 200-E } \\
\text { (PUREX) }\end{array}$ & $\begin{array}{l}46^{\circ} 32^{\prime} 58.1^{n} \text { long. } \\
119^{\circ} 31^{\prime} 14.4^{\prime \prime} \text { lat. }\end{array}$ & $\begin{array}{l}575,100 \mathrm{~m} \mathrm{E} \\
135,640 \mathrm{~m} \mathrm{~N}\end{array}$ & $\begin{array}{l}200 \mathrm{E}(\# 6) \\
1983-1996\end{array}$ \\
\hline $\begin{array}{c}\text { 300-E } \\
\text { (331 Bldg.) }\end{array}$ & $\begin{array}{l}46^{\circ} 21^{\prime} 53.7^{\prime \prime} \text { long. } \\
119 \circ 16^{\prime} 17.3^{\prime \prime} \text { lat. }\end{array}$ & $\begin{array}{l}594,528 \mathrm{~m} \mathrm{E} \\
115,396 \mathrm{~m} \mathrm{~N}\end{array}$ & $\begin{array}{c}300(\# 11) \\
1983-1996\end{array}$ \\
\hline $\begin{array}{c}300-W \\
\text { (324 Bldg.) }\end{array}$ & $\begin{array}{l}46^{\circ} 22^{\prime} 7.8^{\prime \prime} \text { long. } \\
119^{\circ} 16^{\prime} 28.3^{\prime \prime} \text { lat. }\end{array}$ & $\begin{array}{l}594,285 \mathrm{~m} \mathrm{E} \\
115,828 \mathrm{~m} \mathrm{~N}\end{array}$ & $\begin{array}{l}300(\# 11) \\
1983-1996\end{array}$ \\
\hline $\begin{array}{l}400 \text { Area } \\
\text { (FFTF) }\end{array}$ & $\begin{array}{l}46^{\circ} 26^{\prime} 7.0^{\prime \prime} \text { long. } \\
119^{\circ} 21^{\prime} 35.9^{\prime \prime} \text { lat. }\end{array}$ & $\begin{array}{l}587,605 \mathrm{~m} \mathrm{E} \\
123,115 \mathrm{~m} \mathrm{~N}\end{array}$ & $\begin{array}{c}400(\# 9) \\
1983-1996\end{array}$ \\
\hline
\end{tabular}

- The coordinates provided are accurate only to $\pm 100 \mathrm{~m}$.

b Not all meteorological stations have the same amount of data available. The years of available data are specified below each station.

FFTF = Fast Flux Test Facility

PUREX = Plutonium-Uranium Extraction

REDOX $=$ Reduction and Oxidation 
HNF-3602, Vol. 1

Table 4-2. Direction and Distance to each Nearest Offsite Receptor Location from each Release Location.

\begin{tabular}{|c|c|c|}
\hline $\begin{array}{l}\text { Emission zone } \\
\text { (building/facility) }\end{array}$ & Direction & $\begin{array}{c}\text { Distance } \\
\text { (m) }\end{array}$ \\
\hline $\begin{array}{c}100-\mathrm{B} / \mathrm{C} \\
(105-\mathrm{B} \text { Bldg. }\end{array}$ & NNW & 8,600 \\
\hline $\begin{array}{c}100-\mathrm{D} / \mathrm{DR} \\
(105-\mathrm{D} \text { Bldg. })\end{array}$ & WNW & 8,900 \\
\hline $\begin{array}{c}100-\mathrm{F} \\
(105-\mathrm{F} \text { Bldg. }\end{array}$ & ESE & 9,700 \\
\hline $\begin{array}{c}100-H \\
(105-H \text { Bldg. }\end{array}$ & ESE & 11,600 \\
\hline $\begin{array}{c}100-\mathrm{K} \\
(105-\mathrm{KW} \text { Bldg.) }\end{array}$ & NNW & 8,900 \\
\hline $\begin{array}{c}100-N \\
(105-N \text { Bldg) }\end{array}$ & WNW & 8,500 \\
\hline $\begin{array}{c}\text { 200-E } \\
\text { (PUREX) }\end{array}$ & ESE & 20,200 \\
\hline $\begin{array}{c}\text { 200-W } \\
\text { (REDOX) }\end{array}$ & SE & 22,000 \\
\hline $\begin{array}{c}\text { 300-E } \\
\text { (331 Bldg.) }\end{array}$ & $\mathrm{NE}$ & 1,100 \\
\hline $\begin{array}{c}300-W \\
\text { (324 Bldg.) }\end{array}$ & $\mathrm{NE}$ & 1,400 \\
\hline $\begin{array}{l}400 \text { Area } \\
\text { (FFTF) }\end{array}$ & SE & 9,100 \\
\hline
\end{tabular}

2 Distances are the shortest distance to the Hanford Site boundary in a given sector of the wind rose. 
Table 4-3. 100-B/C Area--CAP88-PC Unit Dose Factors by Effective Release Height ${ }^{\mathrm{a}}(\mathrm{mrem} / \mathrm{Ci})$.

\begin{tabular}{|c|c|c|c|c|c|}
\hline \multirow{2}{*}{ Nuclide } & \multicolumn{2}{|c|}{$\begin{array}{c}\text { Effective } \\
\text { release height }\end{array}$} & \multirow{2}{*}{ Nuclide } & \multicolumn{2}{|c|}{$\begin{array}{c}\text { Effective } \\
\text { Release Height }\end{array}$} \\
\hline & $\mathrm{H}_{\mathrm{eff}}<40 \mathrm{~m}$ & $\mathrm{H}_{\text {eff }} \geq 40 \mathrm{~m}$ & & $\mathrm{H}_{\mathrm{eff}}<40 \mathrm{~m}$ & $\mathrm{H}_{\text {eff }} \geq 40 \mathrm{~m}$ \\
\hline $\mathrm{H}-3$ & $5.1 \mathrm{E}-05$ & $2.6 \mathrm{E}-05$ & $\mathrm{Kr}-85$ & $1.1 \mathrm{E}-07$ & $5.8 \mathrm{E}-08$ \\
\hline $\mathrm{Be}-7$ & $4.1 \mathrm{E}-04$ & 2.5 E-04 & $\mathrm{Kr}-85 \mathrm{~m}$ & $4.5 \mathrm{E}-06$ & $2.3 \mathrm{E}-06$ \\
\hline $\mathrm{Be}-10^{\mathrm{b}}$ & $(\mathrm{Sr}-90)$ & $(\mathrm{Sr}-90)$ & $\mathrm{Kr}-87$ & $1.6 \mathrm{E}-05$ & 7.8 E-06 \\
\hline C-11 & $4.1 \mathrm{E}-06$ & 2.3 E-06 & $\mathrm{Kr}-88$ & $5.6 \mathrm{E}-05$ & $2.8 \mathrm{E}-05$ \\
\hline C-14 & $4.0 \mathrm{E}-03$ & $2.0 \mathrm{E}-03$ & $\mathrm{Kr}-89$ & $4.6 \mathrm{E}-08$ & $2.4 \mathrm{E}-08$ \\
\hline $\mathrm{C}-15^{\mathrm{c}}$ & 0.0 & 0.0 & $\mathrm{Kr}-90$ & 2.5 E-19 & $1.3 \mathrm{E}-19$ \\
\hline $\mathrm{N}-13$ & 9.5 E-07 & $5.5 \mathrm{E}-07$ & $\mathrm{Rb}-86$ & $6.0 \mathrm{E}-03$ & $3.7 \mathrm{E}-03$ \\
\hline $0-15$ & $1.0 \mathrm{E}-09$ & $6.1 \mathrm{E}-10$ & $\mathrm{Rb}-87$ & $2.0 \mathrm{E}-02$ & $1.2 \mathrm{E}-02$ \\
\hline F-18 & $2.5 \mathrm{E}-05$ & $1.5 \mathrm{E}-05$ & $\mathrm{Rb}-88$ & 2.7 E-06 & $1.5 \mathrm{E}-06$ \\
\hline $\mathrm{Na}-22$ & $2.7 \mathrm{E}-01$ & $1.7 \mathrm{E}-01$ & $\mathrm{Rb}-89$ & $5.4 \mathrm{E}-06$ & $3.1 \mathrm{E}-06$ \\
\hline $\mathrm{Na}-24$ & $4.6 \mathrm{E}-04$ & $2.8 \mathrm{E}-04$ & Rb-90 & $1.6 \mathrm{E}-08$ & $9.3 \mathrm{E}-09$ \\
\hline P-32 & 6.0 E-03 & $3.8 \mathrm{E}-03$ & $\mathrm{Rb}-90 \mathrm{~m}$ & $2.7 \mathrm{E}-07$ & $1.6 \mathrm{E}-07$ \\
\hline S-35 & 8.5 E-04 & $5.3 \mathrm{E}-04$ & Sr-89 & $3.6 \mathrm{E}-03$ & 2.2 E-03 \\
\hline $\operatorname{Ar}-41$ & $2.8 \mathrm{E}-05$ & $1.4 \mathrm{E}-05$ & Sr-90 & $2.3 \mathrm{E}-01$ & 1.5 E-01 \\
\hline $\mathrm{K}-40$ & $2.6 \mathrm{E}-01$ & $1.6 \mathrm{E}-01$ & Sr-91 & $9.5 \mathrm{E}-05$ & 5.7 E-05 \\
\hline $\mathrm{Ca}-41$ & $2.1 \mathrm{E}-05$ & $1.3 \mathrm{E}-05$ & Sr-92 & 6.1 E-05 & $3.6 \mathrm{E}-05$ \\
\hline Sc-46 & $2.5 \mathrm{E}-02$ & $1.6 \mathrm{E}-02$ & $Y-90$ & $7.2 \mathrm{E}-0.4$ & $4.4 \mathrm{E}-04$ \\
\hline Cr-51 & $1.8 \mathrm{E}-04$ & $1.1 \mathrm{E}-04$ & $\mathrm{Y}-90 \mathrm{~m}$ & $2.1 \mathrm{E}-05$ & $1.3 \mathrm{E}-05$ \\
\hline Mn-54 & $3.4 \mathrm{E}-02$ & $2.1 \mathrm{E}-02$ & Y-91 & $5.3 \mathrm{E}-03$ & $3.2 \mathrm{E}-03$ \\
\hline $\mathrm{Mn}-56$ & $6.8 \mathrm{E}-05$ & $4.0 \mathrm{E}-05$ & $\mathrm{Y}-91 \mathrm{~m}$ & $7.0 \mathrm{E}-06$ & $4.1 \mathrm{E}-06$ \\
\hline $\mathrm{Fe}-55$ & $4.8 \mathrm{E}-04$ & $3.0 \mathrm{E}-04$ & $Y-92$ & $5.2 \mathrm{E}-05$ & $3.1 \mathrm{E}-05$ \\
\hline $\mathrm{Fe}-59$ & $9.8 \mathrm{E}-03$ & $6.1 \mathrm{E}-03$ & $Y-93$ & $1.3 \mathrm{E}-04$ & $7.5 \mathrm{E}-05$ \\
\hline Co-57 & $5.9 \mathrm{E}-03$ & $3.7 \mathrm{E}-03$ & $\mathrm{Zr}-93$ & $2.7 \mathrm{E}-03$ & $1.6 \mathrm{E}-03$ \\
\hline Co-58 & $1.1 \mathrm{E}-02$ & 7.1 E-03 & $\mathrm{Zr}-95$ & $8.1 \mathrm{E}-03$ & $5.1 \mathrm{E}-03$ \\
\hline Co- 60 & 5.1 E-01 & $3.2 \mathrm{E}-01$ & $\mathrm{Nb}-93 \mathrm{~m}$ & $4.3 \mathrm{E}-03$ & $2.7 \mathrm{E}-03$ \\
\hline $\mathrm{Ni}-59$ & $6.4 \mathrm{E}-04$ & $4.0 \mathrm{E}-04$ & $\mathrm{Nb}-94$ & $1.7 \mathrm{E}+\infty$ & $1.1 \mathrm{E}+00$ \\
\hline $\mathrm{Ni}-63$ & $5.5 \mathrm{E}-04$ & 3.4 E-04 & $\mathrm{Nb}-95$ & $1.0 \mathrm{E}-02$ & $6.2 \mathrm{E}-03$ \\
\hline $\mathrm{Ni}-65$ & $2.7 \mathrm{E}-05$ & 1.6 E-05 & $\mathrm{Nb}-95 \mathrm{~m}$ & $6.3 \mathrm{E}-04$ & $3.9 \mathrm{E}-04$ \\
\hline $\mathrm{Cu}-64$ & $3.3 \mathrm{E}-05$ & $2.0 \mathrm{E}-05$ & Nb-97 & $1.4 \mathrm{E}-05$ & $8.5 \mathrm{E}-06$ \\
\hline $\mathrm{Zn}-65$ & $5.1 \mathrm{E}-02$ & $3.2 \mathrm{E}-02$ & $\mathrm{Nb}-97 \mathrm{~m}$ & $1.6 \mathrm{E}-13$ & $9.8 \mathrm{E}-14$ \\
\hline $\mathrm{Zn}-69 \mathrm{~m}$ & 8.7 E-05 & $5.3 \mathrm{E}-05$ & Mo-93 & $5.8 \mathrm{E}-03$ & $3.7 \mathrm{E}-03$ \\
\hline $\mathrm{Zn}-69$ & $1.3 \mathrm{E}-06$ & 7.5 E-07 & Mo-99 & $3.7 \mathrm{E}-04$ & $2.2 \mathrm{E}-04$ \\
\hline Ga-67 & $1.3 \mathrm{E}-04$ & $7.9 \mathrm{E}-05$ & Tc-97 & $1.2 \mathrm{E}-02$ & $7.5 \mathrm{E}-03$ \\
\hline As-76 & $2.9 \mathrm{E}-04$ & $1.7 \mathrm{E}-04$ & Tc-99 & $4.7 \mathrm{E}-02$ & $2.9 \mathrm{E}-02$ \\
\hline $\mathrm{Se}-79^{\mathrm{b}}$ & (Pu-241) & (Pu-241) & Tc-99m & 8.4 E-06 & 5.1 E-06 \\
\hline $\mathrm{Br}-82$ & $6.7 \mathrm{E}-04$ & $4.2 \mathrm{E}-04$ & Tc-101 & $6.7 \mathrm{E}-07$ & $3.9 \mathrm{E}-07$ \\
\hline $\mathrm{Br}-83$ & $2.0 \mathrm{E}-07$ & $1.2 \mathrm{E}-07$ & Ru-97 & $1.4 \mathrm{E}-04$ & 8.6 E-05 \\
\hline $\mathrm{Br}-84$ & $1.4 \mathrm{E}-05$ & $8.2 \mathrm{E}-06$ & Ru-103 & $3.8 \mathrm{E}-03$ & $2.4 \mathrm{E}-03$ \\
\hline $\mathrm{Br}-85$ & $7.3 \mathrm{E}-10$ & 4.4 E-10 & Ru-105 & 5.7 E-05 & $3.4 \mathrm{E}-05$ \\
\hline $\mathrm{Kr}-83 \mathrm{~m}$ & $5.0 \mathrm{E}-09$ & $2.5 \mathrm{E}-09$ & $\mathrm{Ru}-106$ & $3.3 \mathrm{E}-02$ & $2.0 \mathrm{E}-02$ \\
\hline
\end{tabular}


HNF-3602, Vol. 1

Table 4-3. 100-B/C Area--CAP88-PC Unit Dose Factors by Effective Release Height $^{\mathrm{a}}(\mathrm{mrem} / \mathrm{Ci})$.

\begin{tabular}{|c|c|c|c|c|c|}
\hline \multirow{2}{*}{ Nuclide } & \multicolumn{2}{|c|}{$\begin{array}{l}\text { Effective } \\
\text { release height }\end{array}$} & \multirow{2}{*}{ Nuclide } & \multicolumn{2}{|c|}{$\begin{array}{c}\text { Effective } \\
\text { Release Height }\end{array}$} \\
\hline & $\mathrm{H}_{\mathrm{eff}}<40 \mathrm{~m}$ & $\mathrm{H}_{\mathrm{eff}} \geq 40 \mathrm{~m}$ & & $\mathrm{H}_{\mathrm{eff}}<40 \mathrm{~m}$ & $\mathrm{H}_{\text {eff }} \geq 40 \mathrm{~m}$ \\
\hline Rh-103m & $1.7 \mathrm{E}-07$ & $9.5 \mathrm{E}-08$ & I-129 & 2.7 E-01 & $1.2 \mathrm{E}+00$ \\
\hline Rh-105 & $1.0 \mathrm{E}-04$ & $6.2 \mathrm{E}-05$ & I-130 & $9.9 \mathrm{E}-05$ & $4.3 \mathrm{E}-04$ \\
\hline $\mathrm{Rh}-105 \mathrm{~m}$ & $2.5 \mathrm{E}-17$ & $1.5 \mathrm{E}-17$ & I-131 & $8.9 \mathrm{E}-03$ & $3.8 \mathrm{E}-02$ \\
\hline Rh-106 & $2.8 \mathrm{E}-21$ & $1.7 \mathrm{E}-21$ & $\mathrm{I}-132$ & $1.4 \mathrm{E}-05$ & $6.2 \mathrm{E}-05$ \\
\hline Pd-107 & $7.8 \mathrm{E}-04$ & $4.7 \mathrm{E}-04$ & I-133 & $1.0 \mathrm{E}-04$ & $4.4 \mathrm{E}-04$ \\
\hline Pd-109 & $7.6 \mathrm{E}-05$ & $4.5 \mathrm{E}-05$ & I-134 & $4.1 \mathrm{E}-06$ & $1.8 \mathrm{E}-05$ \\
\hline $\mathrm{Ag}-109 \mathrm{~m}$ & 2.0 E-19 & $1.2 \mathrm{E}-19$ & I-135 & $3.3 \mathrm{E}-05$ & $1.4 \mathrm{E}-04$ \\
\hline Ag-110 & $3.2 \mathrm{E}-25$ & $1.9 \mathrm{E}-25$ & $\mathrm{Xe}-122$ & $2.2 \mathrm{E}-06$ & $1.1 \mathrm{E}-06$ \\
\hline $\mathrm{Ag}-110 \mathrm{~m}$ & $1.0 \mathrm{E}-01$ & $6.2 \mathrm{E}-02$ & $\mathrm{Xe}-123$ & $1.4 \mathrm{E}-05$ & $7.2 \mathrm{E}-06$ \\
\hline Ag-111 & $2.1 \mathrm{E}-03$ & $1.3 \mathrm{E}-03$ & $\mathrm{Xe}-125$ & $8.0 \mathrm{E}-06$ & $4.1 \mathrm{E}-06$ \\
\hline $\mathrm{Cd}-113^{\mathrm{b}}$ & (Pu-241) & $(\mathrm{Pu}-241)$ & $\mathrm{Xe}-127$ & $8.9 \mathrm{E}-06$ & $4.5 \mathrm{E}-06$ \\
\hline $\mathrm{Cd}-113 \mathrm{~m}^{\mathrm{b}}$ & (Pu-241) & (Pu-241) & $\mathrm{Xe}-131 \mathrm{~m}$ & $3.2 \mathrm{E}-07$ & $1.7 \mathrm{E}-07$ \\
\hline Cd-115 & 4.1 E-04 & $2.5 \mathrm{E}-04$ & Xe-133 & $1.2 \mathrm{E}-06$ & $6.1 \mathrm{E}-07$ \\
\hline Cd-115m & $7.5 \mathrm{E}-03$ & $4.6 \mathrm{E}-03$ & $\mathrm{Xe}-133 \mathrm{~m}$ & $1.0 \mathrm{E}-06$ & 5.2 E-07 \\
\hline In-113m & $6.9 \mathrm{E}-06$ & $4.1 \mathrm{E}-06$ & $\mathrm{Xe}-135$ & $7.6 \mathrm{E}-06$ & $3.9 \mathrm{E}-06$ \\
\hline In-115 & $1.3 \mathrm{E}-01$ & $7.7 \mathrm{E}-02$ & $\mathrm{Xe}-135 \mathrm{~m}$ & $1.2 \mathrm{E}-06$ & $5.8 \mathrm{E}-07$ \\
\hline In-115m & $1.3 \mathrm{E}-05$ & $8.0 \mathrm{E}-06$ & $\mathrm{Xe}-137$ & $1.1 \mathrm{E}-08$ & $5.7 \mathrm{E}-09$ \\
\hline Sn-113 & 4.6 E-03 & $2.9 \mathrm{E}-03$ & Xe-138 & $3.0 \mathrm{E}-06$ & $1.5 \mathrm{E}-06$ \\
\hline Sn-123 & $1.1 \mathrm{E}-04$ & $6.7 \mathrm{E}-05$ & Cs-134 & $2.1 \mathrm{E}-01$ & $1.3 \mathrm{E}-01$ \\
\hline Sn-125 & $5.0 \mathrm{E}-03$ & $3.1 \mathrm{E}-03$ & Cs-134m & $2.3 \mathrm{E}-06$ & $1.4 \mathrm{E}-06$ \\
\hline $\mathrm{Sn}-126$ & $9.8 \mathrm{E}-02$ & $6.1 \mathrm{E}-02$ & Cs-135 & $9.2 \mathrm{E}-03$ & $5.7 \mathrm{E}-03$ \\
\hline Sb-124 & $1.8 \mathrm{E}-02$ & $1.1 \mathrm{E}-02$ & Cs-136 & $7.7 \mathrm{E}-03$ & $4.8 \mathrm{E}-03$ \\
\hline $\mathrm{Sb}-125$ & $5.5 \mathrm{E}-02$ & $3.4 \mathrm{E}-02$ & Cs-137 & $5.5 \mathrm{E}-02$ & $3.5 \mathrm{E}-02$ \\
\hline Sb-126 & $6.8 \mathrm{E}-03$ & $4.3 \mathrm{E}-03$ & Cs-138 & $2.0 \mathrm{E}-05$ & $1.1 \mathrm{E}-05$ \\
\hline $\mathrm{Sb}-126 \mathrm{~m}$ & 5.7 E-06 & $3.2 \mathrm{E}-06$ & Cs-139 & $2.6 \mathrm{E}-07$ & $1.5 \mathrm{E}-07$ \\
\hline Sb-127 & $9.8 \mathrm{E}-04$ & $6.0 \mathrm{E}-04$ & Ba-133 & $1.6 \mathrm{E}-01$ & 9.7 E-02 \\
\hline $\mathrm{Te}-125 \mathrm{~m}$ & $2.2 \mathrm{E}-03$ & $1.3 \mathrm{E}-03$ & $\mathrm{Ba}-133 \mathrm{~m}$ & $6.6 \mathrm{E}-05$ & $4.0 \mathrm{E}-05$ \\
\hline Te-127 & $1.8 \mathrm{E}-05$ & $1.1 \mathrm{E}-05$ & $\mathrm{Ba}-137 \mathrm{~m}$ & $3.1 \mathrm{E}-09$ & $1.9 \mathrm{E}-09$ \\
\hline $\mathrm{Te}-127 \mathrm{~m}$ & $5.3 \mathrm{E}-03$ & 3.3 E-03 & Ва-139 & $5.5 \mathrm{E}-06$ & $3.2 \mathrm{E}-06$ \\
\hline Te-129 & $3.9 \mathrm{E}-06$ & $2.3 \mathrm{E}-06$ & Ba-140 & $2.2 \mathrm{E}-03$ & $1.4 \mathrm{E}-03$ \\
\hline $\mathrm{Te}-129 \mathrm{~m}$ & $5.2 \mathrm{E}-03$ & $3.2 \mathrm{E}-03$ & Ba-141 & $2.9 \mathrm{E}-06$ & $1.6 \mathrm{E}-06$ \\
\hline Te-131 & $3.2 \mathrm{E}-06$ & $1.8 \mathrm{E}-06$ & Ba-142 & $1.0 \mathrm{E}-06$ & $5.8 \mathrm{E}-07$ \\
\hline $\mathrm{Te}-131 \mathrm{~m}$ & $5.0 \mathrm{E}-04$ & $3.0 \mathrm{E}-04$ & La-140 & $8.4 \mathrm{E}-04$ & $5.2 \mathrm{E}-04$ \\
\hline $\mathrm{Te}-132$ & $8.6 \mathrm{E}-04$ & $5.3 \mathrm{E}-04$ & La-141 & $1.6 \mathrm{E}-06$ & 9.5 E-07 \\
\hline Te-133 & $1.4 \mathrm{E}-06$ & 8.2 E-07 & La-142 & $6.1 \mathrm{E}-05$ & $3.6 \mathrm{E}-05$ \\
\hline $\mathrm{Te}-133 \mathrm{~m}$ & $3.0 \mathrm{E}-05$ & $1.8 \mathrm{E}-05$ & Ce-141 & $1.6 \mathrm{E}-03$ & $9.7 \mathrm{E}-04$ \\
\hline Te-134 & 8.7 E-06 & $5.0 \mathrm{E}-06$ & Ce-143 & $2.5 \mathrm{E}-04$ & $1.5 \mathrm{E}-04$ \\
\hline I-122 & $1.7 \mathrm{E}-09$ & $6.6 \mathrm{E}-09$ & Ce-144 & $2.6 \mathrm{E}-02$ & $1.6 \mathrm{E}-02$ \\
\hline $\mathrm{I}-123$ & $9.1 \mathrm{E}-06$ & $3.9 \mathrm{E}-05$ & Pr-143 & $1.2 \mathrm{E}-03$ & $7.6 \mathrm{E}-04$ \\
\hline I-125 & $1.6 \mathrm{E}-02$ & $6.9 \mathrm{E}-02$ & Pr-144 & $4.8 \mathrm{E}-07$ & $2.7 \mathrm{E}-07$ \\
\hline
\end{tabular}


Table 4-3. 100-B/C Area--CAP88-PC Unit Dose Factors by Effective Release Height ${ }^{\text {a }}(\mathrm{mrem} / \mathrm{Ci})$.

\begin{tabular}{|c|c|c|c|c|c|}
\hline \multirow{2}{*}{ Nuclide } & \multicolumn{2}{|c|}{$\begin{array}{c}\text { Effective } \\
\text { release height }\end{array}$} & \multirow{2}{*}{ Nuclide } & \multicolumn{2}{|c|}{$\begin{array}{c}\text { Effective } \\
\text { Release Height }\end{array}$} \\
\hline & $\mathrm{H}_{\mathrm{eff}}<40 \mathrm{~m}$ & $\mathrm{H}_{\mathrm{eff}} \geq 40 \mathrm{~m}$ & & $\mathrm{H}_{\mathrm{eff}}<40 \mathrm{~m}$ & $\mathrm{H}_{\text {eff }} \geq 40 \mathrm{~m}$ \\
\hline Pr-144m & $2.5 \mathrm{E}-08$ & $1.5 \mathrm{E}-08$ & Po-211 ${ }^{\mathrm{c}}$ & 0.0 & 0.0 \\
\hline Nd-147 & $1.2 \mathrm{E}-03$ & 7.5 E-04 & $\mathrm{Po}-212^{\mathrm{c}}$ & 0.0 & 0.0 \\
\hline Pm-147 & $2.3 \mathrm{E}-03$ & $1.4 \mathrm{E}-03$ & Po- $213^{\mathrm{c}}$ & 0.0 & 0.0 \\
\hline Pm-148 & $1.8 \mathrm{E}-03$ & $1.1 \mathrm{E}-03$ & Po- $214^{\mathrm{C}}$ & 0.0 & 0.0 \\
\hline Pm-148m & $1.6 \mathrm{E}-02$ & $1.0 \mathrm{E}-02$ & Po- $215^{\mathfrak{C}}$ & 0.0 & 0.0 \\
\hline Pm-149 & $2.3 \mathrm{E}-04$ & 1.4 E-04 & Po-216 & 0.0 & 0.0 \\
\hline Pm-151 & 6.1 E-05 & $3.8 \mathrm{E}-05$ & Po-218 & $1.4 \mathrm{E}-09$ & 8.1 E-10 \\
\hline $\mathrm{Sm}-147$ & $3.5 \mathrm{E}+00$ & $2.1 \mathrm{E}+\infty$ & At-217 ${ }^{\mathrm{C}}$ & 0.0 & 0.0 \\
\hline Sm-151 & $1.6 \mathrm{E}-03$ & $9.3 \mathrm{E}-04$ & $R n-219^{d}$ & $(\mathrm{~Pb}-21 \mathrm{i})$ & $(\mathrm{Pb}-211)$ \\
\hline Sm-153 & $1.6 \mathrm{E}-04$ & $9.8 \mathrm{E}-05$ & $R n-220^{d}$ & $(\mathrm{~Pb}-212)$ & $(\mathrm{Pb}-212)$ \\
\hline $\mathrm{Eu}-152$ & $5.0 \mathrm{E}-01$ & $3.1 \mathrm{E}-01$ & Rn-222 & $1.4 \mathrm{E}-04$ & $7.3 \mathrm{E}-05$ \\
\hline $\mathrm{Eu}-152 \mathrm{~m}$ & $2.2 \mathrm{E}-05$ & $1.3 \mathrm{E}-05$ & Fr-221 & 8.4 E-07 & 5.0 E-07 \\
\hline Eu-154 & $4.1 \mathrm{E}-01$ & $2.6 \mathrm{E}-01$ & Fr-223 & $1.9 \mathrm{E}-05$ & 1.1 E-05 \\
\hline Eu-155 & $1.7 \mathrm{E}-02$ & $1.0 \mathrm{E}-02$ & $\mathrm{Ra}-223$ & $5.0 \mathrm{E}-01$ & 3.0 E-01 \\
\hline Eu-156 & $5.1 \mathrm{E}-03$ & $3.1 \mathrm{E}-03$ & Ra-224 & $1.8 \mathrm{E}-01$ & $1.1 \mathrm{E}-01$ \\
\hline $\mathrm{Gd}-152^{b}$ & (Pu-239) & (Pu-239) & $\mathrm{Ra}-225$ & $3.1 \mathrm{E}-01$ & $1.9 \mathrm{E}-01$ \\
\hline Tb-160 & $1.3 \mathrm{E}-02$ & $8.3 \mathrm{E}-03$ & $\mathrm{Ra}-226$ & $9.6 \mathrm{E}-01$ & 5.9 E-01 \\
\hline Ho-166 & $1.9 \mathrm{E}-04$ & $1.1 \mathrm{E}-04$ & $\mathrm{Ra}-228$ & $4.0 \mathrm{E}-01$ & $2.4 \mathrm{E}-01$ \\
\hline Ho-166m & $1.7 \mathrm{E}+\infty 0$ & $1.0 \mathrm{E}+00$ & Ac-225 & $3.0 \mathrm{E}-01$ & $1.8 \mathrm{E}-01$ \\
\hline Hf-181 & $5.1 \mathrm{E}-03$ & $3.2 \mathrm{E}-03$ & Ac-227 & $3.1 \mathrm{E}+01$ & $1.8 \mathrm{E}+01$ \\
\hline W-181 & 9.4 E-04 & $5.9 \mathrm{E}-04$ & Ac-228 & $3.5 \mathrm{E}-03$ & $2.0 \mathrm{E}-03$ \\
\hline W-185 & $1.3 \mathrm{E}-03$ & $7.8 \mathrm{E}-04$ & Th-227 & $5.5 \mathrm{E}-01$ & 3.3 E-01 \\
\hline W-187 & $1.1 \mathrm{E}-04$ & $6.6 \mathrm{E}-05$ & Th-228 & $1.2 \mathrm{E}+01$ & $6.9 \mathrm{E}+00$ \\
\hline Re-187 & $3.6 \mathrm{E}-05$ & $2.2 \mathrm{E}-05$ & Th-229 & $3.3 \mathrm{E}+01$ & $1.9 \mathrm{E}+01$ \\
\hline Ir-192 & $1.1 \mathrm{E}-02$ & $7.1 \mathrm{E}-03$ & Th-230 & $1.2 \mathrm{E}+01$ & $6.9 \mathrm{E}+00$ \\
\hline $\mathrm{Hg}-203$ & $7.1 \mathrm{E}-03$ & $4.4 \mathrm{E}-03$ & Th-231 & $5.1 \mathrm{E}-05$ & 3.0 E-05 \\
\hline Tl-207 & 1.8 E-09 & $1.1 \mathrm{E}-09$ & Th-232 & $1.7 E+01$ & $9.9 \mathrm{E}+00$ \\
\hline T1-208 & $5.9 \mathrm{E}-08$ & $3.5 \mathrm{E}-08$ & Th-234 & $5.0 \mathrm{E}-03$ & $3.1 \mathrm{E}-03$ \\
\hline Tl-209 & $4.0 \mathrm{E}-09$ & $2.4 \mathrm{E}-09$ & $\mathrm{~Pa}-231$ & $2.4 \mathrm{E}+01$ & $1.4 \mathrm{E}+01$ \\
\hline $\mathrm{Pb}-209$ & $3.5 \mathrm{E}-06$ & $2.1 \mathrm{E}-06$ & $\mathrm{~Pa}-233$ & $2.2 \mathrm{E}-03$ & $1.3 \mathrm{E}-03$ \\
\hline $\begin{array}{l}\mathrm{Pb}-210 \\
\mathrm{~Pb}-211\end{array}$ & $\begin{array}{c}3.0 \mathrm{E}+00 \\
1.2 \mathrm{E}-04\end{array}$ & $\begin{array}{c}1.8 \mathrm{E}+\infty \\
7.1 \mathrm{E}-05\end{array}$ & $\begin{array}{c}\mathrm{Pa}-234 \\
\mathrm{~Pa}-234 \mathrm{~m}\end{array}$ & $\begin{array}{l}1.5 \mathrm{E}-04 \\
4.4 \mathrm{E}-14\end{array}$ & $\begin{array}{l}9.2 \text { E-05 } \\
2.6 \text { E-14 }\end{array}$ \\
\hline $\mathrm{Pb}-212$ & $7.2 \mathrm{E}-03$ & $4.2 \mathrm{E}-03$ & U-232 & $2.3 \mathrm{E}+01$ & $1.4 \mathrm{E}+01$ \\
\hline $\mathrm{Pb}-214$ & $1.0 \mathrm{E}-05$ & 5.7 E-06 & U-233 & $6.6 \mathrm{E}+\infty 0$ & $3.9 \mathrm{E}+00$ \\
\hline $\mathrm{Bi}-210$ & 9.5 E-03 & $5.6 \mathrm{E}-03$ & U-234 & $6.5 \mathrm{E}+\infty$ & $3.9 \mathrm{E}+00$ \\
\hline $\mathrm{Bi}-211$ & 2.7 E-09 & 1.6 E-09 & U-235 & $6.2 \mathrm{E}+00$ & $3.7 \mathrm{E}+00$ \\
\hline $\mathrm{Bi}-212$ & $6.9 \mathrm{E}-04$ & $4.0 \mathrm{E}-04$ & U-236 & $6.2 E+00$ & $3.7 E+\infty 0$ \\
\hline $\mathrm{Bi}-213$ & $2.2 \mathrm{E}-05$ & $1.3 \mathrm{E}-05$ & U-237 & $6.4 \mathrm{E}-04$ & $4.0 \mathrm{E}-04$ \\
\hline $\mathrm{Bi}-214$ & $8.2 \mathrm{E}-06$ & $4.7 \mathrm{E}-06$ & U-238. & $5.8 \mathrm{E}+00$ & $3.4 \mathrm{E}+00$ \\
\hline Po-210 & $1.1 \mathrm{E}+\infty$ & $6.7 \mathrm{E}-01$ & $\mathrm{U}-240$ & $1.1 \mathrm{E}-04$ & $6.6 \mathrm{E}-05$ \\
\hline
\end{tabular}


HNF-3602, Vol. 1

Table 4-3. 100-B/C Area-CAP88-PC Unit Dose Factors by Effective Release Height $^{\mathrm{a}}$ (mrem/Ci).

\begin{tabular}{|c|c|c|c|c|c|}
\hline \multirow{2}{*}{ Nuclide } & \multicolumn{2}{|c|}{$\begin{array}{c}\text { Effective } \\
\text { release height }\end{array}$} & \multirow{2}{*}{ Nuclide } & \multicolumn{2}{|c|}{$\begin{array}{c}\text { Effective } \\
\text { Release Height }\end{array}$} \\
\hline & $\mathrm{H}_{\mathrm{eff}}<40 \mathrm{~m}$ & $\mathrm{H}_{\text {eff }} \geq 40 \mathrm{~m}$ & & $\mathrm{H}_{\text {eff }}<40 \mathrm{~m}$ & $\mathrm{H}_{\text {eff }} \geq 40 \mathrm{~m}$ \\
\hline Np-237 & $2.4 \mathrm{E}+01$ & $1.4 \mathrm{E}+01$ & & & \\
\hline Np-238 & $1.7 \mathrm{E}-03$ & $1.0 \mathrm{E}-03$ & & & \\
\hline Np-239 & $2.5 \mathrm{E}-04$ & $1.5 \mathrm{E}-04$ & & & \\
\hline $\mathrm{Np}-240$ & $2.1 \mathrm{E}-05$ & $1.2 \mathrm{E}-05$ & & & \\
\hline $\mathrm{Np}-240 \mathrm{~m}$ & $1.6 \mathrm{E}-07$ & $9.3 \mathrm{E}-08$ & & & \\
\hline Pu-236 & $4.2 \mathrm{E}+00$ & $2.5 \mathrm{E}+\infty$ & & & \\
\hline Pu-238 & $1.6 \mathrm{E}+01$ & $9.5 \mathrm{E}+00$ & & & \\
\hline $\mathrm{Pu}-239$ & $1.7 \mathrm{E}+01$ & $1.0 \mathrm{E}+01$ & & & \\
\hline $\mathrm{Pu}-240$ & $1.7 \mathrm{E}+01$ & $1.0 \mathrm{E}+01$ & & & \\
\hline Pu-241 & $2.7 \mathrm{E}-01$ & $1.6 \mathrm{E}-01$ & & & \\
\hline $\mathrm{Pu}-242$ & $1.6 \mathrm{E}+01$ & $9.8 \mathrm{E}+00$ & & & \\
\hline $\mathrm{Pu}-243$ & $1.0 \mathrm{E}-05$ & $5.9 \mathrm{E}-06$ & & & \\
\hline $\mathrm{Pu}-244$ & $1.6 \mathrm{E}+01$ & $9.7 \mathrm{E}+00$ & & & \\
\hline Am-241 & $2.7 \mathrm{E}+01$ & $1.6 \mathrm{E}+01$ & & & \\
\hline Am-242 & $2.6 \mathrm{E}-03$ & $1.5 \mathrm{E}-03$ & & & \\
\hline $\mathrm{Am}-242 \mathrm{~m}$ & $2.6 \mathrm{E}+01$ & $1.5 \mathrm{E}+01$ & & & \\
\hline Am-243 & $2.7 \mathrm{E}+01$ & $1.6 \mathrm{E}+01$ & & & \\
\hline $\mathrm{Cm}-242$ & $8.6 \mathrm{E}-01$ & $5.1 \mathrm{E}-01$ & & & \\
\hline $\mathrm{Cm}-243$ & $1.8 \mathrm{E}+01$ & $1.1 \mathrm{E}+01$ & & & \\
\hline $\mathrm{Cm}-244$ & $1.4 \mathrm{E}+01$ & $8.3 E+\infty 0$ & & & \\
\hline $\mathrm{Cm}-245$ & $2.8 \mathrm{E}+01$ & $1.6 \mathrm{E}+01$ & & & \\
\hline $\mathrm{Cm}-246$ & $2.7 \mathrm{E}+01$ & $1.6 \mathrm{E}+01$ & & & \\
\hline $\mathrm{Cm}-247$ & $2.5 \mathrm{E}+01$ & $1.5 \mathrm{E}+01$ & & & \\
\hline $\mathrm{Cm}-248$ & $1.0 \mathrm{E}+02$ & $5.9 \mathrm{E}+01$ & & & \\
\hline Cf-252 & $7.5 \mathrm{E}+00$ & $4.4 \mathrm{E}+00$ & & & \\
\hline
\end{tabular}

2 The effective release height, $x$, is in meters.

b CAP88-PC Ver. 1.0 does not calculate doses for Be-10, Se-79, Cd-113, Cd-113m, and Gd-152. Recommend substituting the unit dose factors of the nuclides in parentheses.

c Extremely short lived nuclide, resulting in an offsite dose of zero.

d Short lived radon nuclides only provide the mechanism to circumvent the emission control device. The unit dose factors for the recommended progeny should be used instead. However, 1 ci of $\mathrm{Rn}$ does not equal $1 \mathrm{ci}$ of $\mathrm{Pb}$. Multiply the activity of $\mathrm{Rn}-219$ by 0.0018 to get the activity of $\mathrm{Pb}-211$. Multiply the activity of $\mathrm{Rn}-220$ by 0.0014 to get the activity of $\mathrm{Pb}-212$. 
Table 4-4. 100-D/DR Area--CAP88-PC Unit Dose Factors by Effective Release Height ${ }^{\mathrm{a}}$ (mrem/Ci).

\begin{tabular}{|c|c|c|c|c|c|}
\hline \multirow{2}{*}{ Nuclide } & \multicolumn{2}{|c|}{$\begin{array}{c}\text { Effective } \\
\text { Release Height }\end{array}$} & \multirow{2}{*}{ Nuclide } & \multicolumn{2}{|c|}{$\begin{array}{c}\text { Effective } \\
\text { Release Height }\end{array}$} \\
\hline & $\mathrm{H}_{\mathrm{eff}}<40 \mathrm{~m}$ & $\mathrm{H}_{\text {eff }} \geq 40 \mathrm{~m}$ & & $\mathrm{H}_{\mathrm{eff}}<40 \mathrm{~m}$ & $\mathrm{H}_{\text {eff }} \geq 40 \mathrm{~m}$ \\
\hline $\mathrm{H}-3$ & $3.8 \mathrm{E}-05$ & $2.2 \mathrm{E}-05$ & $\mathrm{Kr}-85$ & $8.4 \mathrm{E}-08$ & $4.9 \mathrm{E}-08$ \\
\hline $\mathrm{Be}-7$ & $2.8 \mathrm{E}-04$ & $2.1 \mathrm{E}-04$ & $\mathrm{Kr}-85 \mathrm{~m}$ & $3.0 \mathrm{E}-06$ & $1.8 \mathrm{E}-06$ \\
\hline $\mathrm{Be}-10^{\mathrm{b}}$ & $(\mathrm{SI}-90)$ & $(\mathrm{Sr}-90)$ & $\mathrm{Kr}-87$ & $8.5 \mathrm{E}-06$ & $5.1 \mathrm{E}-06$ \\
\hline C-11 & $1.5 \mathrm{E}-06$ & $1.1 \mathrm{E}-06$ & $\mathrm{Kr}-88$ & $3.6 \mathrm{E}-05$ & 2.1E-05 \\
\hline C-14 & $2.9 \mathrm{E}-03$ & $1.7 \mathrm{E}-03$ & $\mathrm{Kr}-89$ & $2.5 \mathrm{E}-08$ & $1.5 \mathrm{E}-08$ \\
\hline $\mathrm{C}-15^{\mathrm{C}}$ & 0.0 & 0.0 & $\mathrm{Kr}-90$ & $5.6 \mathrm{E}-20$ & $3.5 \mathrm{E}-20$ \\
\hline N-13 & $4.2 \mathrm{E}-07$ & $3.1 \mathrm{E}-07$ & $\mathrm{Rb}-86$ & $4.1 \mathrm{E}-\mathrm{C} 3$ & $3.1 \mathrm{E}-03$ \\
\hline $0-15$ & $4.7 \mathrm{E}-10$ & $3.5 \mathrm{E}-10$ & $R b+87$ & $1.4 \mathrm{E}-02$ & $1.0 \mathrm{E}-02$ \\
\hline F-18 & $1.4 \mathrm{E}-05$ & $1.0 \mathrm{E}-05$ & $\mathrm{Rb}-88$ & $9.8 \mathrm{E}-07$ & 7.3E-07 \\
\hline $\mathrm{Na}-22$ & $1.8 \mathrm{E}-01$ & $1.4 \mathrm{E}-01$ & $\mathrm{Rb}-89$ & $2.0 \mathrm{E}-06$ & $1.5 \mathrm{E}-06$ \\
\hline $\mathrm{Na}-24$ & $3.1 \mathrm{E}-04$ & $2.3 \mathrm{E}-04$ & $\mathrm{Rb}-90$ & $7.5 \mathrm{E}-09$ & $5.6 \mathrm{E}-09$ \\
\hline P-32 & $4.2 \mathrm{E}-03$ & $3.1 \mathrm{E}-03$ & $\mathrm{Rb}-90 \mathrm{~m}$ & $1.4 \mathrm{E}-07$ & $1.1 \mathrm{E}-07$ \\
\hline S-35 & $5.9 \mathrm{E}-04$ & $4.4 \mathrm{E}-04$ & Sr-89 & $2.5 \mathrm{E}-03$ & $1.8 \mathrm{E}-03$ \\
\hline Ar-41 & $1.7 \mathrm{E}-05$ & $9.9 \mathrm{E}-06$ & Sr-90 & $1.6 \mathrm{E}-01$ & $1.2 \mathrm{E}-01$ \\
\hline $\mathrm{K}-40$ & $1.8 \mathrm{E}-01$ & $1.3 \mathrm{E}-01$ & Sr-91 & $6.2 \mathrm{E}-05$ & 4.6E-05 \\
\hline $\mathrm{Ca}-41$ & $1.5 \mathrm{E}-05$ & $1.1 \mathrm{E}-05$ & Sr-92 & $3.6 \mathrm{E}-05$ & $2.6 \mathrm{E}-05$ \\
\hline Sc-46 & $1.7 \mathrm{E}-02$ & $1.3 \mathrm{E}-02$ & Y-90 & $4.9 \mathrm{E}-04$ & $3.6 \mathrm{E}-04$ \\
\hline $\mathrm{Cr}-51$ & $1.3 \mathrm{E}-04$ & $9.4 \mathrm{E}-05$ & Y-90m & $1.3 \mathrm{E}-05$ & $9.5 \mathrm{E}-06$ \\
\hline$M n-54$ & $2.4 \mathrm{E}-02$ & $1.8 \mathrm{E}-02$ & Y-91 & $3.6 \mathrm{E}-03$ & $2.7 \mathrm{E}-03$ \\
\hline $\mathrm{Mn}-56$ & $4.0 \mathrm{E}-05$ & $2.9 \mathrm{E}-05$ & Y-91m & $3.1 \mathrm{E}-06$ & $2.3 \mathrm{E}-06$ \\
\hline Fe-55 & $3.3 \mathrm{E}-04$ & $2.5 \mathrm{E}-04$ & Y-92 & $3.2 \mathrm{E}-05$ & $2.3 \mathrm{E}-05$ \\
\hline Fe-59 & $6.8 \mathrm{E}-03$ & $5.1 \mathrm{E}-03$ & $Y-93$ & $8.3 \mathrm{E}-05$ & $6.0 \mathrm{E}-05$ \\
\hline Co-57 & $4.1 \mathrm{E}-03$ & $3.1 \mathrm{E}-03$ & Zr-93 & $1.8 \mathrm{E}-03$ & $1.3 \mathrm{E}-03$ \\
\hline $\mathrm{Co}-58$ & $7.8 \mathrm{E}-03$ & $5.9 \mathrm{E}-03$ & $\mathrm{Zr}-95$ & $5.6 \mathrm{E}-03$ & $4.2 \mathrm{E}-03$ \\
\hline $\mathrm{Co}-60$ & $3.6 \mathrm{E}-01$ & 2.7E-01 & $\mathrm{Nb}-93 \mathrm{~m}$ & $3.0 \mathrm{E}-03$ & $2.2 \mathrm{E}-03$ \\
\hline $\mathrm{Ni}-59$ & 4.4E-04 & $3.3 \mathrm{E}-04$ & $\mathrm{Nb}-94$ & $1.2 \mathrm{E}+\infty 0$ & $8.7 \mathrm{E}-01$ \\
\hline $\mathrm{Ni}-63$ & $3.8 \mathrm{E}-04$ & $2.8 \mathrm{E}-04$ & $\mathrm{Nb}-95$ & $6.9 \mathrm{E}-03$ & $5.2 \mathrm{E}-03$ \\
\hline $\mathrm{Ni}-65$ & $1.6 \mathrm{E}-05$ & $1.2 \mathrm{E}-05$ & $\mathrm{Nb}-95 \mathrm{~m}$ & 4.3E-04 & $3.2 \mathrm{E}-04$ \\
\hline $\mathrm{Cu}-64$ & $2.2 \mathrm{E}-05$ & $1.6 \mathrm{E}-05$ & $\mathrm{Nb}-97$ & $7.3 \mathrm{E}-06$ & 5.3E-06 \\
\hline $\mathrm{Zn}-65$ & $3.6 \mathrm{E}-02$ & $2.7 \mathrm{E}-02$ & $\mathrm{Nb}-97 \mathrm{~m}$ & $5.6 \mathrm{E}-14$ & $4.2 \mathrm{E}-14$ \\
\hline $\mathrm{Zn}-69 \mathrm{~m}$ & $5.8 \mathrm{E}-05$ & $4.3 \mathrm{E}-05$ & Mo-93 & $4.0 \mathrm{E}-03$ & $3.0 \mathrm{E}-03$ \\
\hline Zn-69 & $6.0 \mathrm{E}-07$ & $4.4 \mathrm{E}-07$ & Mo-99 & $2.5 \mathrm{E}-04$ & $1.8 \mathrm{E}-04$ \\
\hline Ga-67 & $8.8 \mathrm{E}-05$ & $6.5 \mathrm{E}-05$ & Tc-97 & $8.3 \mathrm{E}-03$ & $6.2 \mathrm{E}-03$ \\
\hline As-76 & $2.0 \mathrm{E}-04$ & $1.4 \mathrm{E}-04$ & Tc-99 & $3.2 \mathrm{E}-02$ & $2.4 \mathrm{E}-02$ \\
\hline $\mathrm{Se}-79^{b}$ & (Pu-241) & (Pu-241) & Tc-99m & $5.4 \mathrm{E}-06$ & $4.0 \mathrm{E}-06$ \\
\hline $\mathrm{Br}-82$ & $4.6 \mathrm{E}-04$ & $3.4 \mathrm{E}-04$ & Tc-101 & $2.6 \mathrm{E}-07$ & $1.9 \mathrm{E}-07$ \\
\hline $\mathrm{Br}-83$ & $1.2 \mathrm{E}-07$ & $8.7 \mathrm{E}-08$ & Ru-97 & $9.5 \mathrm{E}-05$ & 7.1E-05 \\
\hline $\mathrm{Br}-84$ & $5.6 \mathrm{E}-06$ & $4.1 \mathrm{E}-06$ & $\mathrm{Ru}-103$ & $2.7 \mathrm{E}-03$ & $2.0 \mathrm{E}-03$ \\
\hline $\mathrm{Br}-85$ & $3.6 \mathrm{E}-10$ & $2.7 \mathrm{E}-10$ & $\mathrm{Ru}-105$ & $3.6 \mathrm{E}-05$ & $2.6 \mathrm{E}-05$ \\
\hline $\mathrm{Kr}-83 \mathrm{~m}$ & $3.0 \mathrm{E}-09$ & $1.8 \mathrm{E}-09$ & $\mathrm{Ru}-106$ & $2.2 \mathrm{E}-02$ & $1.6 \mathrm{E}-02$ \\
\hline
\end{tabular}


Table 4-4. 100-D/DR Area--CAP88-PC Unit Dose Factors by Effective Release Height $^{\mathrm{a}}(\mathrm{mrem} / \mathrm{Ci})$.

\begin{tabular}{|c|c|c|c|c|c|}
\hline \multirow{2}{*}{ Nuclide } & \multicolumn{2}{|c|}{$\begin{array}{c}\text { Effective } \\
\text { Release Height }\end{array}$} & \multirow{2}{*}{ Nuclide } & \multicolumn{2}{|c|}{$\begin{array}{c}\text { Effective } \\
\text { Release Height }\end{array}$} \\
\hline & $\mathrm{H}_{\mathrm{eff}}<40 \mathrm{~m}$ & $\mathrm{H}_{\text {eff }} \geq 40 \mathrm{~m}$ & & $\mathrm{H}_{\mathrm{eff}}<40 \mathrm{~m}$ & $\mathrm{H}_{\text {eff }} \geq 40 \mathrm{~m}$ \\
\hline $\mathrm{Rh}-103 \mathrm{~m}$ & 7.7E-08 & $5.6 \mathrm{E}-08$ & $\mathrm{I}-129$ & $1.5 \mathrm{E}-01$ & $6.1 \mathrm{E}-01$ \\
\hline Rh-105 & $6.9 \mathrm{E}-05$ & $5.1 \mathrm{E}-05$ & I-130 & $5.4 \mathrm{E}-05$ & $2.2 \mathrm{E}-04$ \\
\hline $\mathrm{Rh}-105 \mathrm{~m}$ & $7.1 \mathrm{E}-18$ & $5.3 \mathrm{E}-18$ & $\mathrm{I}-131$ & $5.0 \mathrm{E}-03$ & $2.0 \mathrm{E}-02$ \\
\hline Rh-106 & $5.3 \mathrm{E}-22$ & $3.9 \mathrm{E}-22$ & I-132 & 7.2E-06 & $2.8 \mathrm{E}-05$ \\
\hline $\mathrm{Pd}-107$ & $5.4 \mathrm{E}-04$ & $3.9 \mathrm{E}-04$ & I-133 & $5.6 \mathrm{E}-05$ & 2.3E-04 \\
\hline Pd-109 & $5.0 \mathrm{E}-05$ & $3.6 \mathrm{E}-05$ & I-134 & $1.8 \mathrm{E}-06$ & $6.7 \mathrm{E}-06$ \\
\hline $\mathrm{Ag}-109 \mathrm{~m}$ & $5.1 \mathrm{E}-20$ & $3.8 \mathrm{E}-20$ & I-135 & $1.8 \mathrm{E}-05$ & $7.1 \mathrm{E}-05$ \\
\hline Ag-110 & $4.7 \mathrm{E}-26$ & $3.5 \mathrm{E}-26$ & $\mathrm{Xe}-122$ & $1.6 \mathrm{E}-06$ & $9.3 \mathrm{E}-07$ \\
\hline $\mathrm{Ag}-110 \mathrm{~m}$ & $6.9 \mathrm{E}-02$ & $5.2 \mathrm{E}-02$ & $\mathrm{Xe}-123$ & $8.7 \mathrm{E}-06$ & $5.2 \mathrm{E}-06$ \\
\hline Ag- 111 & $1.5 \mathrm{E}-03$ & $1.1 \mathrm{E}-03$ & $\mathrm{Xe}-125$ & $5.7 \mathrm{E}-06$ & $3.4 \mathrm{E}-06$ \\
\hline $\mathrm{Cd}-113^{\mathrm{b}}$ & (Pu-241) & $(\mathrm{Pu}-241)$ & $\mathrm{Xe}-127$ & $6.5 \mathrm{E}-06$ & $3.8 \mathrm{E}-06$ \\
\hline $\mathrm{Cd}-113 \mathrm{~m}^{\mathrm{b}}$ & (Pu-241) & (Pu-241) & $\mathrm{Xe}-131 \mathrm{~m}$ & $2.4 \mathrm{E}-07$ & $1.4 \mathrm{E}-07$ \\
\hline Cd-115 & $2.8 \mathrm{E}-04$ & $2.0 \mathrm{E}-04$ & $\mathrm{Xe}-133$ & $8.7 E-07$ & $5.2 \mathrm{E}-07$ \\
\hline $\mathrm{Cd}-115 \mathrm{~m}$ & $5.1 \mathrm{E}-03$ & $3.8 \mathrm{E}-03$ & $\mathrm{Xe}-133 \mathrm{~m}$ & 7.4E-07 & $4.4 \mathrm{E}-07$ \\
\hline In-113m & $3.8 \mathrm{E}-06$ & $2.8 \mathrm{E}-06$ & $\mathrm{Xe}-135$ & $5.3 \mathrm{E}-06$ & $3.2 \mathrm{E}-06$ \\
\hline In-115 & 8.7E-02 & $6.4 \mathrm{E}-02$ & $\mathrm{Xe}-135 \mathrm{~m}$ & 4.7E-07 & $2.9 \mathrm{E}-07$ \\
\hline In-115m & 8.4E-06 & $6.1 \mathrm{E}-06$ & $\mathrm{Xe}-137$ & $6.0 \mathrm{E}-09$ & $3.8 \mathrm{E}-09$ \\
\hline Sn-113 & 3.2E-03 & $2.4 \mathrm{E}-03$ & Xe-138 & $1.2 \mathrm{E}-06$ & 7.4E-07 \\
\hline $\mathrm{Sn}-123$ & 7.4E-05 & $5.6 \mathrm{E}-05$ & Cs-134 & $1.4 \mathrm{E}-01$ & $1.1 \mathrm{E}-01$ \\
\hline Sn-125 & $3.4 \mathrm{E}-03$ & $2.5 \mathrm{E}-03$ & Cs $-134 \mathrm{~m}$ & $1.4 \mathrm{E}-06$ & $9.9 \mathrm{E}-07$ \\
\hline Sn-126 & $6.8 \mathrm{E}-02$ & $5.1 \mathrm{E}-02$ & Cs-135 & $6.4 \mathrm{E}-03$ & $4.8 \mathrm{E}-03$ \\
\hline Sb-124 & $1.2 \mathrm{E}-02$ & $9.3 \mathrm{E}-03$ & Cs-136 & $5.3 \mathrm{E}-03$ & $4.0 \mathrm{E}-03$ \\
\hline Sb-125 & $3.8 \mathrm{E}-02$ & $2.8 \mathrm{E}-02$ & Cs-137 & $3.8 \mathrm{E}-02$ & $2.9 \mathrm{E}-02$ \\
\hline Sb-126 & $4.7 \mathrm{E}-03$ & $3.5 \mathrm{E}-03$ & Cs-138 & 7.7E-06 & $5.7 \mathrm{E}-06$ \\
\hline $\mathrm{Sb}-126 \mathrm{~b}$ & $2.1 \mathrm{E}-06$ & $1.5 \mathrm{E}-06$ & Cs-139 & $1.2 \mathrm{E}-07$ & $8.8 \mathrm{E}-08$ \\
\hline $\mathrm{Sb}-127$ & $6.7 \mathrm{E}-04$ & $5.0 \mathrm{E}-04$ & Ba-133 & $1.1 \mathrm{E}-01$ & $8.1 \mathrm{E}-02$ \\
\hline $\mathrm{Te}-125 \mathrm{~m}$ & $1.5 \mathrm{E}-03$ & $1.1 \mathrm{E}-03$ & Ba-133m & 4.5E-05 & 3.3E-05 \\
\hline Te-127 & $1.2 \mathrm{E}-05$ & $8.6 \mathrm{E}-06$ & $\mathrm{Ba}-137 \mathrm{~m}$ & $1.5 \mathrm{E}-09$ & $1.1 \mathrm{E}-09$ \\
\hline Te-127m & $3.7 \mathrm{E}-03$ & $2.7 \mathrm{E}-03$ & Ba-139 & $2.9 \mathrm{E}-06$ & $2.1 \mathrm{E}-06$ \\
\hline $\mathrm{Te}-129$ & $1.9 \mathrm{E}-06$ & $1.4 \mathrm{E}-06$ & $\mathrm{Ba}-140$ & $1.5 \mathrm{E}-03$ & 1.1E-03 \\
\hline $\mathrm{Te}-129 \mathrm{~m}$ & $3.6 \mathrm{E}-03$ & $2.7 \mathrm{E}-03$ & $\mathrm{Ba}-141$ & $1.0 \mathrm{E}-06$ & $7.8 \mathrm{E}-07$ \\
\hline $\mathrm{Te}-131$ & $1.2 \mathrm{E}-06$ & $8.8 \mathrm{E}-07$ & Ba-142 & $4.2 \mathrm{E}-07$ & $3.2 \mathrm{E}-07$ \\
\hline $\mathrm{Te}-131 \mathrm{~m}$ & $3.4 \mathrm{E}-04$ & $2.5 \mathrm{E}-04$ & La-140 & $5.8 \mathrm{E}-04$ & $4.2 \mathrm{E}-04$ \\
\hline Te-132 & $5.9 \mathrm{E}-04$ & $4.3 \mathrm{E}-04$ & La-141 & $9.8 \mathrm{E}-07$ & 7.2E-07 \\
\hline Te-133 & $5.7 \mathrm{E}-07$ & $4.3 \mathrm{E}-07$ & La-142 & 3.3E-05 & $2.4 \mathrm{E}-05$ \\
\hline $\mathrm{Te}-133 \mathrm{~m}$ & $1.4 \mathrm{E}-05$ & $1.0 \mathrm{E}-05$ & Ce-141 & $1.1 \mathrm{E}-03$ & $8.0 \mathrm{E}-04$ \\
\hline $\mathrm{Te}-134$ & $3.7 \mathrm{E}-06$ & $2.7 \mathrm{E}-06$ & $\mathrm{Ce}-143$ & $1.7 \mathrm{E}-04$ & $1.2 \mathrm{E}-04$ \\
\hline $\mathrm{I}-122$ & 7.4E-10 & $2.7 \mathrm{E}-09$ & $\mathrm{Ce}-144$ & $1.8 \mathrm{E}-02$ & $1.3 E-02$ \\
\hline I-123 & $5.0 \mathrm{E}-06$ & $2.0 \mathrm{E}-05$ & Pr-143 & $8.5 \mathrm{E}-04$ & $6.3 \mathrm{E}-0.4$ \\
\hline I-125 & $9.0 \mathrm{E}-03$ & $3.6 \mathrm{E}-02$ & $\operatorname{Pr}-144$ & $1.7 \mathrm{E}-07$ & $1.3 \mathrm{E}-07$ \\
\hline
\end{tabular}


HNF-3602, Vol. 1

Table 4-4. 100-D/DR Area--CAP88-PC Unit Dose Factors by Effective Release Height $^{\mathrm{a}}$ (mrem/Ci).

\begin{tabular}{|c|c|c|c|c|c|}
\hline \multirow{2}{*}{ Nuclide } & \multicolumn{2}{|c|}{$\begin{array}{c}\text { Effective } \\
\text { Release Height }\end{array}$} & \multirow{2}{*}{ Nuclide } & \multicolumn{2}{|c|}{$\begin{array}{c}\text { Effective } \\
\text { Release Height }\end{array}$} \\
\hline & $\mathrm{H}_{\mathrm{eff}}<40 \mathrm{~m}$ & $\mathrm{H}_{\text {eff }} \geq 40 \mathrm{~m}$ & & $\mathrm{H}_{\text {eff }}<40 \mathrm{~m}$ & $\mathrm{H}_{\text {eff } Z} 40 \mathrm{~m}$ \\
\hline Pr-144m & $1.3 \mathrm{E}-08$ & 9.4E-09 & Po-211 & 0.0 & 0.0 \\
\hline Nd-147 & 8.3E-04 & $6.2 \mathrm{E}-04$ & Po-212 & 0.0 & 0.0 \\
\hline Pm-147 & $1.5 \mathrm{E}-03$ & $1.1 \mathrm{E}-03$ & $\mathrm{Po}-213^{\mathrm{c}}$ & 0.0 & 0.0 \\
\hline Pm-148 & $1.2 \mathrm{E}-03$ & $9.2 \mathrm{E}-04$ & $\mathrm{Po}-214^{\mathrm{C}}$ & 0.0 & 0.0 \\
\hline Pm-148m & $1.1 \mathrm{E}-02$ & $8.3 E-03$ & Po- $215^{\mathrm{c}}$ & 0.0 & 0.0 \\
\hline Pm-149 & $1.6 \mathrm{E}-04$ & $1.1 \mathrm{E}-04$ & Po- $216^{\mathrm{c}}$ & 0.0 & 0.0 \\
\hline Pm-151 & $4.1 \mathrm{E}-05$ & 3.1E-05 & Po-218 & $6.8 \mathrm{E}-10$ & $5.1 \mathrm{E}-10$ \\
\hline Sm-147 & $2.4 \mathrm{E}+00$ & $1.7 \mathrm{E}+\infty 0$ & At $-217^{\mathrm{c}}$ & 0.0 & 0.0 \\
\hline Sm-151 & $1.1 \mathrm{E}-03$ & $7.7 \mathrm{E}-04$ & Rn-219 & $(\mathrm{Pb}-211)$ & $(\mathrm{Pb}-211)$ \\
\hline $\mathrm{Sm}-153$ & $1.1 \mathrm{E}-04$ & $8.0 \mathrm{E}-05$ & $R n-220^{d}$ & $(\mathrm{~Pb}-212)$ & $(\mathrm{Pb}-212)$ \\
\hline Eu-152 & $3.5 \mathrm{E}-01$ & $2.6 \mathrm{E}-01$ & $\mathrm{Rn}-222$ & $1.1 \mathrm{E}-04$ & $6.2 \mathrm{E}-05$ \\
\hline $\mathrm{Eu}-152 \mathrm{~m}$ & $1.5 \mathrm{E}-05$ & $1.1 \mathrm{E}-05$ & Fr-221 & $4.4 \mathrm{E}-07$ & $3.3 \mathrm{E}-07$ \\
\hline Eu-154 & $2.8 \mathrm{E}-01$ & $2.1 \mathrm{E}-01$ & Fr-223 & $6.7 \mathrm{E}-06$ & $5.0 \mathrm{E}-06$ \\
\hline Eu-155 & $1.1 \mathrm{E}-02$ & $8.5 \mathrm{E}-03$ & $\mathrm{Ra}-223$ & $3.4 \mathrm{E}-01$ & $2.5 \mathrm{E}-01$ \\
\hline Eu-156 & $3.5 \mathrm{E}-03$ & $2.6 \mathrm{E}-03$ & $\mathrm{Ra}-224$ & $1.2 \mathrm{E}-01$ & $8.8 \mathrm{E}-02$ \\
\hline $\mathrm{Gd}-152^{\mathrm{b}}$ & (Pu-239) & (Pu-239) & $\mathrm{Ra}-225$ & $2.1 \mathrm{E}-01$ & $1.6 \mathrm{E}-01$ \\
\hline $\mathrm{Tb}-160$ & $9.2 \mathrm{E}-03$ & $6.9 \mathrm{E}-03$ & $\mathrm{Ra}-226$ & $6.6 \mathrm{E}-01$ & $4.8 \mathrm{E}-01$ \\
\hline Ho-166 & $1.3 \mathrm{E}-04$ & $9.2 \mathrm{E}-05$ & $\mathrm{Ra}-228$ & $2.7 \mathrm{E}-01$ & $2.0 \mathrm{E}-01$ \\
\hline Ho-166m & $1.1 \mathrm{E}+00$ & $8.6 \mathrm{E}-01$ & Ac-225 & $2.0 \mathrm{E}-01$ & $1.5 \mathrm{E}-01$ \\
\hline Hf-181 & $3.5 \mathrm{E}-03$ & $2.6 \mathrm{E}-03$ & Ac-227 & $2.1 E+01$ & $1.5 \mathrm{E}+01$ \\
\hline W-181 & $6.5 \mathrm{E}-04$ & $4.9 \mathrm{E}-04$ & Ac- 228 & $2.2 \mathrm{E}-03$ & $1.6 \mathrm{E}-03$ \\
\hline W-185 & $8.6 \mathrm{E}-04$ & $6.4 \mathrm{E}-04$ & Th-227 & $3.7 \mathrm{E}-01$ & $2.7 \mathrm{E}-01$ \\
\hline W-187 & $7.3 \mathrm{E}-05$ & $5.4 \mathrm{E}-05$ & Th-228 & $8.0 \mathrm{E}+\infty 0$ & $5.7 \mathrm{E}+\infty 0$ \\
\hline $\operatorname{Re}-187$ & $2.5 \mathrm{E}-05$ & $1.9 \mathrm{E}-05$ & Th-229 & $2.2 \mathrm{E}+01$ & $1.6 \mathrm{E}+01$ \\
\hline Ir-192 & $7.8 \mathrm{E}-03$ & $5.8 \mathrm{E}-03$ & Th-230 & $8.0 \mathrm{E}+\infty 0$ & $5.7 \mathrm{E}+00$ \\
\hline $\mathrm{Hg}-203$ & $4.9 \mathrm{E}-03$ & $3.6 \mathrm{E}-03$ & Th-231 & $3.4 \mathrm{E}-05$ & $2.5 \mathrm{E}-05$ \\
\hline Tl-207 & $9.4 \mathrm{E}-10$ & 7.0E-10 & Th-232 & $1.1 \mathrm{E}+01$ & $8.2 \mathrm{E}+\infty 0$ \\
\hline Tl-208 & $3.0 \mathrm{E}-08$ & 2.2E-08 & Th-234 & $3.4 \mathrm{E}-03$ & 2.5E-03 \\
\hline T1-209 & $1.9 \mathrm{E}-09$ & $1.4 \mathrm{E}-09$ & $\mathrm{~Pa}-231$ & $1.6 \mathrm{E}+01$ & $1.2 \mathrm{E}+01$ \\
\hline $\mathrm{Pb}-209$ & $2.1 \mathrm{E}-06$ & $1.5 \mathrm{E}-06$ & $\mathrm{~Pa}-233$ & $1.5 \mathrm{E}-03$ & 1.1E-03 \\
\hline $\mathrm{Pb}-210$ & $2.0 \mathrm{E}+\infty 0$ & $1.5 \mathrm{E}+\infty 0$ & $\mathrm{~Pa}-234$ & $9.9 \mathrm{E}-05$ & $7.2 \mathrm{E}-05$ \\
\hline $\mathrm{Pb}-211$ & $5.0 \mathrm{E}-05$ & $3.7 \mathrm{E}-05$ & $\mathrm{~Pa}-234 \mathrm{~m}$ & $1.6 \mathrm{E}-14$ & $1.2 \mathrm{E}-14$ \\
\hline $\mathrm{Pb}-212$ & 4.7E-03 & $3.4 \mathrm{E}-03$ & U-232 & $1.6 \mathrm{E}+01$ & $1.1 \mathrm{E}+01$ \\
\hline $\mathrm{Pb}-214$ & $3.7 \mathrm{E}-06$ & $2.8 \mathrm{E}-06$ & U-233 & $4.5 \mathrm{E}+00$ & $3.2 \mathrm{E}+00$ \\
\hline $\mathrm{Bi}-210$ & $6.4 \mathrm{E}-03$ & $4.6 \mathrm{E}-03$ & U-234 & $4.4 E+00$ & $3.2 \mathrm{E}+00$ \\
\hline $\mathrm{Bi}-211$ & $1.3 \mathrm{E}-09$ & $9.4 \mathrm{E}-10$ & U-235 & $4.2 \mathrm{E}+00$ & $3.1 \mathrm{E}+00$ \\
\hline $\mathrm{Bi}-212$ & 3.3E-04 & $2.4 \mathrm{E}-04$ & U-236 & $4.2 \mathrm{E}+00$ & $3.0 \mathrm{E}+00$ \\
\hline $\mathrm{Bi}-213$ & 9.4E-06 & $6.9 \mathrm{E}-06$ & U-237 & $4.4 \mathrm{E}-04$ & $3.3 \mathrm{E}-04$ \\
\hline $\mathrm{Bi}-214$ & $3.0 \mathrm{E}-06$ & 2.2E-06 & $\mathrm{U}-238$ & $3.9 \mathrm{E}+00$ & $2.8 \mathrm{E}+00$ \\
\hline Po-210 & 7.5E-01 & $5.5 \mathrm{E}-01$ & $\mathrm{U}-240$ & 7.3E-05 & $5.3 \mathrm{E}-05$ \\
\hline
\end{tabular}


Table 4-4. 100-D/DR Area--CAP88-PC Unit Dose Factors by Effective Release Height $^{\mathrm{a}}(\mathrm{mrem} / \mathrm{Ci})$.

\begin{tabular}{|c|c|c|c|c|c|}
\hline \multirow{2}{*}{ Nuclide } & \multicolumn{2}{|c|}{$\begin{array}{c}\text { Effective } \\
\text { Release Height }\end{array}$} & \multirow{2}{*}{ Nuclide } & \multicolumn{2}{|c|}{$\begin{array}{c}\text { Effective } \\
\text { Release Height }\end{array}$} \\
\hline & $\mathrm{H}_{\mathrm{eff}}<40 \mathrm{~m}$ & $\mathrm{H}_{\text {eff }} \geq 40 \mathrm{~m}$ & & $\mathrm{H}_{\mathrm{eff}}<40 \mathrm{~m}$ & $\mathrm{H}_{\mathrm{eff}} \geq 40 \mathrm{~m}$ \\
\hline Np-237 & $1.7 \mathrm{E}+01$ & $1.2 \mathrm{E}+01$ & & & \\
\hline Np-238 & $1.1 \mathrm{E}-03$ & $8.2 \mathrm{E}-04$ & & & \\
\hline Np-239 & $1.7 \mathrm{E}-04$ & $1.3 \mathrm{E}-04$ & & & \\
\hline $\mathrm{Np}-240$ & $1.0 \mathrm{E}-05$ & $7.4 \mathrm{E}-06$ & & & \\
\hline $\mathrm{Np}-240 \mathrm{~m}$ & 7.7E-08 & $5.8 \mathrm{E}-08$ & & & \\
\hline $\mathrm{Pu}-236$ & $2.9 \mathrm{E}+00$ & $2.1 E+\infty 0$ & & & \\
\hline $\mathrm{Pu}-238$ & $1.1 \mathrm{E}+01$ & $7.9 \mathrm{E}+\infty 0$ & & & \\
\hline $\mathrm{Pu}-239$ & $1.2 \mathrm{E}+01$ & $8.5 \mathrm{E}+\infty 0$ & & & \\
\hline $\mathrm{Pu}-240$ & $1.2 \mathrm{E}+01$ & $8.5 \mathrm{E}+\infty$ & & & \\
\hline Pu-241 & $1.9 \mathrm{E}-01$ & $1.3 \mathrm{E}-01$ & & & \\
\hline $\mathrm{Pu}-242$ & $1.1 \mathrm{E}+01$ & $8.1 E+\infty 0$ & & & \\
\hline Pu-243 & $6.3 \mathrm{E}-06$ & $4.5 \mathrm{E}-06$ & & & \\
\hline $\mathrm{Pu}-244$ & $1.1 \mathrm{E}+01$ & $8.0 \mathrm{E}+\infty 0$ & & & \\
\hline Am-241 & $1.8 \mathrm{E}+01$ & $1.3 \mathrm{E}+01$ & & & . \\
\hline Am-242 & $1.7 \mathrm{E}-03$ & $1.2 \mathrm{E}-03$ & & & \\
\hline$A m-242 m$ & $1.7 \mathrm{E}+01$ & $1.3 \mathrm{E}+.01$ & & & . \\
\hline Am-243 & $1.8 \mathrm{E}+01$ & $1.3 \mathrm{E}+01$ & & & \\
\hline $\mathrm{Cm}-242$ & $5.9 \mathrm{E}-01$ & 4.2E-01 & & & \\
\hline $\mathrm{Cm}-243$ & $1.2 \mathrm{E}+01$ & $8.7 \mathrm{E}+00$ & & & \\
\hline $\mathrm{Cm}-244$ & $9.5 E+00$ & $6.9 \mathrm{E}+00$ & & & \\
\hline $\mathrm{Cm}-245$ & $1.9 \mathrm{E}+01$ & $1.4 \mathrm{E}+01$ & & & \\
\hline $\mathrm{Cm}-246$ & $1.9 \mathrm{E}+01$ & $1.3 \mathrm{E}+01$ & & & \\
\hline $\mathrm{Cm}-247$ & $1.7 \mathrm{E}+01$ & $1.2 \mathrm{E}+01$ & & & \\
\hline $\mathrm{Cm}-248$ & $6.8 \mathrm{E}+01$ & $4.9 \mathrm{E}+01$ & & & \\
\hline Cf-252 & $5.1 \mathrm{E}+00$ & $3.7 \mathrm{E}+\infty 0$ & & & \\
\hline
\end{tabular}

- The effective release height, $x$, is in meters.

b CAP88-PC Ver. 1.0 does not calculate doses for Be-10, Se-79, Cd-113, Cd-113m, and Gd-152. Recommend substituting the unit dose factors of the nuclides in parentheses.

c Extremely short lived nuclide, resulting in an offsite dose of zero.

d Short lived radon nuclides only provide the mechanism to circumvent the emission control device. The unit dose factors for the recommended progeny should be used instead.

However, 1 ci of $\mathrm{Rn}$ does not equal $1 \mathrm{ci}$ of $\mathrm{Pb}$. Multiply the activity of $\mathrm{Rn}-219$ by 0.0018 to get the activity of $\mathrm{Pb}-211$. Multiply the activity of $\mathrm{Rn}-220$ by 0.0014 to get the activity of $\mathrm{Pb}-212$. 
Table 4-5. 100-F Area--CAP88-PC Unit Dose Factors by Effective Release Height ${ }^{\mathrm{a}}$ (mrem/Ci).

\begin{tabular}{|c|c|c|c|c|c|}
\hline \multirow{2}{*}{ Nuclide } & \multicolumn{2}{|c|}{$\begin{array}{c}\text { Effective } \\
\text { Release Height }\end{array}$} & \multirow{2}{*}{ Nuclide } & \multicolumn{2}{|c|}{$\begin{array}{c}\text { Effective } \\
\text { Release Height }\end{array}$} \\
\hline & $\mathrm{H}_{\mathrm{eff}}<40 \mathrm{~m}$ & $\mathrm{H}_{\text {eff }} \geq 40 \mathrm{~m}$ & & $\mathrm{H}_{\text {eff }}<40 \mathrm{~m}$ & $\mathrm{H}_{\text {eff }} \geq 40 \mathrm{~m}$ \\
\hline $\mathrm{H}-3$ & $6.5 \mathrm{E}-05$ & $3.2 \mathrm{E}-05$ & $\mathrm{Kr}-85$ & $1.5 \mathrm{E}-07$ & $7.1 \mathrm{E}-08$ \\
\hline $\mathrm{Be}-7$ & $4.5 \mathrm{E}-04$ & $3.0 \mathrm{E}-04$ & $\mathrm{Kr}-85 \mathrm{~m}$ & $5.2 \mathrm{E}-06$ & $2.6 \mathrm{E}-06$ \\
\hline $\mathrm{Be}-10^{\mathrm{b}}$ & $(\mathrm{Sr}-90)$ & (SI-90) & $\mathrm{Kr}-87$ & $1.5 \mathrm{E}-05$ & $8.6 \mathrm{E}-06$ \\
\hline $\mathrm{C}-11$ & $3.9 \mathrm{E}-06$ & $3.2 \mathrm{E}-06$ & $\mathrm{Kr}-88$ & $6.2 \mathrm{E}-05$ & $3.2 \mathrm{E}-05$ \\
\hline C-14 & $5.1 \mathrm{E}-03$ & $2.5 \mathrm{E}-03$ & $\mathrm{Kr}-89$ & $4.6 \mathrm{E}-08$ & $3.4 \mathrm{E}-08$ \\
\hline$C-15^{\mathfrak{c}}$ & 0.0 & 0.0 & $\mathrm{Kr}-90$ & $1.0 \mathrm{E}-20$ & 7.3E-21 \\
\hline N-13 & $1.2 \mathrm{E}-06$ & $1.0 \mathrm{E}-06$ & $\mathrm{Rb}-86$ & $6.6 \mathrm{E}-\mathrm{C} 3$ & $4.4 \mathrm{E}-03$ \\
\hline $0-15$ & 7.2E-10 & $6.1 \mathrm{E}-10$ & $\mathrm{Rb}-87$ & $2.2 \mathrm{E}-02$ & $1.5 \mathrm{E}-02$ \\
\hline F-18 & $2.4 \mathrm{E}-05$ & $1.6 \mathrm{E}-05$ & $\mathrm{Rb}-88$ & $2.7 \mathrm{E}-06$ & $2.3 \mathrm{E}-06$ \\
\hline $\mathrm{Na}-22$ & 3.0E-01 & $2.0 \mathrm{E}-01$ & $\mathrm{Rb}-89$ & $5.8 \mathrm{E}-06$ & $4.9 \mathrm{E}-06$ \\
\hline $\mathrm{Na}-24$ & $4.9 \mathrm{E}-04$ & $3.3 \mathrm{E}-04$ & Rb-90 & $1.4 \mathrm{E}-08$ & $1.2 \mathrm{E}-08$ \\
\hline P-32 & $6.7 \mathrm{E}-03$ & $4.5 \mathrm{E}-03$ & Rb-90m & $3.3 \mathrm{E}-07$ & $2.8 \mathrm{E}-07$ \\
\hline S-35 & $9.5 \mathrm{E}-04$ & $6.4 \mathrm{E}-04$ & Sr-89 & $4.0 \mathrm{E}-03$ & $2.7 \mathrm{E}-03$ \\
\hline $\mathrm{Ar}-41$ & $2.9 \mathrm{E}-05$ & $1.6 \mathrm{E}-05$ & Sr-90 & $2.6 \mathrm{E}-01$ & $1.7 \mathrm{E}-01$ \\
\hline $\mathrm{K}-40$ & $2.9 \mathrm{E}-01$ & $1.9 \mathrm{E}-01$ & Sr-91 & $1.0 \mathrm{E}-04$ & $6.7 \mathrm{E}-05$ \\
\hline $\mathrm{Ca}-41$ & $2.4 \mathrm{E}-05$ & $1.6 \mathrm{E}-05$ & Sr-92 & $6.0 \mathrm{E}-05$ & $4.1 \mathrm{E}-05$ \\
\hline Sc-46 & $2.8 \mathrm{E}-02$ & $1.9 \mathrm{E}-02$ & $Y-90$ & $8.0 \mathrm{E}-04$ & $5.2 \mathrm{E}-04$ \\
\hline Cr-51 & $2.0 \mathrm{E}-04$ & $1.4 \mathrm{E}-04$ & $Y-90 \mathrm{~m}$ & 2.1E-05 & $1.4 \mathrm{E}-05$ \\
\hline $\mathrm{Mn}-54$ & $3.8 \mathrm{E}-02$ & $2.6 \mathrm{E}-02$ & Y-91 & $5.9 \mathrm{E}-03$ & $3.9 \mathrm{E}-03$ \\
\hline $\mathrm{Mn}-56$ & $6.6 \mathrm{E}-05$ & 4.5E-05 & Y-91m & $5.9 \mathrm{E}-06$ & 4.5E-06 \\
\hline $\mathrm{Fe}-55$ & $5.3 \mathrm{E}-04$ & $3.5 \mathrm{E}-04$ & Y-92 & $5.2 \mathrm{E}-05$ & $3.5 \mathrm{E}-05$ \\
\hline $\mathrm{Fe}-59$ & $1.1 \mathrm{E}-02$ & $7.3 E-03$ & Y-93 & $1.4 \mathrm{E}-04$ & $8.7 \mathrm{E}-05$ \\
\hline $\mathrm{Co}-57$ & $6.6 \mathrm{E}-03$ & $4.4 \mathrm{E}-03$ & ZI-93 & $3.0 \mathrm{E}-03$ & $1.9 \mathrm{E}-03$ \\
\hline Co-58 & $1.3 \mathrm{E}-02$ & $8.4 \mathrm{E}-03$ & Zr-95 & $9.0 \mathrm{E}-03$ & $6.0 \mathrm{E}-03$ \\
\hline Co- 60 & $5.7 \mathrm{E}-01$ & $3.8 \mathrm{E}-01$ & $\mathrm{Nb}-93 \mathrm{~m}$ & $4.8 \mathrm{E}-03$ & $3.2 \mathrm{E}-03$ \\
\hline $\mathrm{Ni}-59$ & 7.1E-04 & $4.8 \mathrm{E}-04$ & $\mathrm{Nb}-94$ & $1.9 \mathrm{E}+00$ & $1.3 E+00$ \\
\hline $\mathrm{Ni}-63$ & $6.1 \mathrm{E}-04$ & $4.0 \mathrm{E}-04$ & $\mathrm{Nb}-95$ & $1.1 \mathrm{E}-02$ & 7.4E-03 \\
\hline $\mathrm{Ni}-65$ & $2.6 \mathrm{E}-05$ & $1.8 \mathrm{E}-05$ & $\mathrm{Nb}-95 \mathrm{~m}$ & $6.9 \mathrm{E}-04$ & $4.6 \mathrm{E}-04$ \\
\hline $\mathrm{Cu}-64$ & $3.5 \mathrm{E}-05$ & 2.3E-05 & $\mathrm{Nb}-97$ & $1.3 \mathrm{E}-05$ & $9.2 \mathrm{E}-06$ \\
\hline $\mathrm{Zn}-65$ & $5.7 \mathrm{E}-02$ & $3.8 \mathrm{E}-02$ & $\mathrm{Nb}-97 \mathrm{~m}$ & $4.0 \mathrm{E}-14$ & $3.3 \mathrm{E}-14$ \\
\hline $\mathrm{Zn}-69 \mathrm{~m}$ & 9.4E-05 & $6.2 \mathrm{E}-05$ & Mo-93 & $6.5 \mathrm{E}-03$ & 4.4E-03 \\
\hline $\mathrm{Zn}-69$ & $1.1 \mathrm{E}-06$ & $8.2 \mathrm{E}-07$ & Mo-99 & $4.0 \mathrm{E}-04$ & $2.6 \mathrm{E}-04$ \\
\hline Ga-67 & $1.4 \mathrm{E}-04$ & $9.4 \mathrm{E}-05$ & Tc-97 & $1.3 \mathrm{E}-02$ & $8.9 \mathrm{E}-03$ \\
\hline As-76 & $3.2 \mathrm{E}-04$ & 2.1E-04 & Tc-99 & $5.2 \mathrm{E}-02$ & $3.5 \mathrm{E}-02$ \\
\hline $\mathrm{Se}-79^{\mathrm{b}}$ & (Pu-241) & (Pu-241) & $\mathrm{Tc}-99 \mathrm{~m}$ & $8.8 \mathrm{E}-06$ & $5.9 \mathrm{E}-06$ \\
\hline $\mathrm{Br}-82$ & 7.4E-04 & $4.9 \mathrm{E}-04$ & Tc-101 & 7.5E-07 & $6.3 \mathrm{E}-07$ \\
\hline $\mathrm{Br}-83$ & $2.0 \mathrm{E}-07$ & $1.4 \mathrm{E}-07$ & Ru-97 & $1.5 \mathrm{E}-04$ & $1.0 \mathrm{E}-04$ \\
\hline $\mathrm{Br}-84$ & $1.2 \mathrm{E}-05$ & $9.8 \mathrm{E}-06$ & Ru-103 & 4.3E-03 & $2.8 \mathrm{E}-03$ \\
\hline $\mathrm{Br}-85$ & 7.0E-10 & $5.9 \mathrm{E}-10$ & Ru-105 & $5.9 \mathrm{E}-05$ & $3.9 \mathrm{E}-05$ \\
\hline $\mathrm{Kr}-83 \mathrm{~m}$ & $5.2 \mathrm{E}-09$ & $2.8 \mathrm{E}-09$ & Ru-106 & $3.6 \mathrm{E}-02$ & $2.4 \mathrm{E}-02$ \\
\hline
\end{tabular}


Table 4-5. 100-F Area--CAP88-PC Unit Dose Factors by Effective Release Height ${ }^{\mathrm{a}}$ $(\mathrm{mrem} / \mathrm{Ci})$.

\begin{tabular}{|c|c|c|c|c|c|}
\hline \multirow{2}{*}{ Nuclide } & \multicolumn{2}{|c|}{$\begin{array}{c}\text { Effective } \\
\text { Release Height }\end{array}$} & \multirow{2}{*}{ Nuclide } & \multicolumn{2}{|c|}{$\begin{array}{c}\text { Effective } \\
\text { Release Height }\end{array}$} \\
\hline & $\mathrm{H}_{\mathrm{eff}}<40 \mathrm{~m}$ & $\mathrm{H}_{\text {eff }} \geq 40 \mathrm{~m}$ & & $\mathrm{H}_{\mathrm{eff}}<40 \mathrm{~m}$ & $\mathrm{H}_{\text {eff }} \geq 40 \mathrm{~m}$ \\
\hline $\mathrm{Rh}-103 \mathrm{~m}$ & $1.4 \mathrm{E}-07$ & $1.1 \mathrm{E}-07$ & I-129 & $4.0 \mathrm{E}-01$ & $1.2 \mathrm{E}+\infty 0$ \\
\hline Rh-105 & $1.1 \mathrm{E}-04$ & $7.4 \mathrm{E}-05$ & $\mathrm{I}-130$ & $1.5 \mathrm{E}-04$ & $4.3 \mathrm{E}-04$ \\
\hline $\mathrm{Rh}-105 \mathrm{~m}$ & $3.0 \mathrm{E}-18$ & $2.6 \mathrm{E}-18$ & $\mathrm{I}-131$ & $1.3 \mathrm{E}-02$ & $3.9 \mathrm{E}-02$ \\
\hline Rh-106 & $8.0 \mathrm{E}-23$ & $6.7 \mathrm{E}-23$ & $\mathrm{I}-132$ & $2.3 \mathrm{E}-05$ & $6.1 \mathrm{E}-05$ \\
\hline Pd-107 & $8.7 \mathrm{E}-04$ & $5.6 \mathrm{E}-04$ & I-133 & $1.5 \mathrm{E}-04$ & 4.4E-04 \\
\hline Pd-109 & $8.2 \mathrm{E}-05$ & $5.3 \mathrm{E}-05$ & I-134 & $7.8 \mathrm{E}-06$ & $1.9 \mathrm{E}-05$ \\
\hline $\mathrm{Ag}-109 \mathrm{~m}$ & $1.7 \mathrm{E}-20$ & $1.4 \mathrm{E}-20$ & I-135 & $4.9 \mathrm{E}-05$ & $1.4 \mathrm{E}-04$ \\
\hline $\mathrm{Ag}-110$ & 3.7E-27 & $3.1 \mathrm{E}-27$ & $\mathrm{Xe}-122$ & $2.7 \mathrm{E}-06$ & $1.3 \mathrm{E}-06$ \\
\hline $\mathrm{Ag}-110 \mathrm{~m}$ & $1.1 \mathrm{E}-01$ & $7.5 \mathrm{E}-02$ & $\mathrm{Xe}-123$ & $1.5 \mathrm{E}-05$ & $8.1 \mathrm{E}-06$ \\
\hline Ag-111 & $2.3 \mathrm{E}-03$ & $1.6 \mathrm{E}-03$ & $\mathrm{Xe}-125$ & $9.9 \mathrm{E}-06$ & $4.9 \mathrm{E}-06$ \\
\hline $\mathrm{Cd}-113^{\mathrm{b}}$ & (Pu-241) & (Pu-241) & $\mathrm{Xe}-127$ & $1.1 \mathrm{E}-05$ & $5.5 \mathrm{E}-06$ \\
\hline$C d-113 m^{b}$ & (Pu-241) & (Pu-241) & $\mathrm{Xe}-131 \mathrm{~m}$ & 4.1E-07 & $2.0 \mathrm{E}-07$ \\
\hline Cd-115 & $4.5 \mathrm{E}-04$ & $2.9 \mathrm{E}-04$ & $X e-133$ & $1.5 \mathrm{E}-06$ & 7.3E-07 \\
\hline Cd-115m & $8.3 \mathrm{E}-03$ & $5.5 \mathrm{E}-03$ & $\mathrm{Xe}-133 \mathrm{~m}$ & $1.3 \mathrm{E}-06$ & $6.3 \mathrm{E}-07$ \\
\hline In-113m & $6.4 \mathrm{E}-06$ & $4.5 \mathrm{E}-06$ & $\mathrm{Xe}-135$ & $9.3 \mathrm{E}-06$ & $4.6 \mathrm{E}-06$ \\
\hline In-115 & $1.4 \mathrm{E}-01$ & $9.2 \mathrm{E}-02$ & $\mathrm{Xe}-135 \mathrm{~m}$ & $1.3 \mathrm{E}-06$ & $9.2 \mathrm{E}-07$ \\
\hline In-115m & $1.4 \mathrm{E}-05$ & $9.1 \mathrm{E}-06$ & Xe-137 & $1.3 \mathrm{E}-08$ & $9.1 \mathrm{E}-09$ \\
\hline Sn-113 & $5.1 \mathrm{E}-03$ & $3.4 \mathrm{E}-03$ & $\mathrm{Xe}-138$ & $3.2 \mathrm{E}-06$ & $2.4 \mathrm{E}-06$ \\
\hline Sn-123 & $1.2 \mathrm{E}-04$ & $8.0 \mathrm{E}-05$ & Cs-134 & 2.3E-01 & $1.6 \mathrm{E}-01$ \\
\hline Sn-125 & $5.5 \mathrm{E}-03$ & $3.7 \mathrm{E}-03$ & $\mathrm{Cs}-134 \mathrm{~m}$ & $2.3 \mathrm{E}-06$ & $1.5 \mathrm{E}-06$ \\
\hline Sn-126 & $1.1 \mathrm{E}-01$ & $7.3 \mathrm{E}-02$ & Cs- 135 & $1.0 \mathrm{E}-02$ & $6.8 \mathrm{E}-03$ \\
\hline $\mathrm{Sb}-124$ & $2.0 \mathrm{E}-02$ & $1.3 \mathrm{E}-02$ & Cs-136 & $8.6 \mathrm{E}-03$ & $5.7 \mathrm{E}-03$ \\
\hline Sb-125 & $6.1 \mathrm{E}-02$ & 4.1E-02 & Cs-137 & $6.1 \mathrm{E}-02$ & 4.1E-02 \\
\hline $\mathrm{Sb}-126$ & $7.6 \mathrm{E}-03$ & $5.1 \mathrm{E}-03$ & Cs-138 & $1.7 \mathrm{E}-05$ & $1.3 \mathrm{E}-05$ \\
\hline$S b-126 b$ & $5.5 \mathrm{E}-06$ & 4.7E-06 & Cs-139 & $3.4 \mathrm{E}-07$ & $2.9 \mathrm{E}-07$ \\
\hline $\mathrm{Sb}-127$ & $1.1 \mathrm{E}-03$ & 7.2E-04 & Ba-133 & $1.7 \mathrm{E}-01$ & $1.2 \mathrm{E}-01$ \\
\hline Te-125m & $2.4 \mathrm{E}-03$ & $1.6 \mathrm{E}-03$ & $\mathrm{Ba}-133 \mathrm{~m}$ & 7.3E-05 & $4.8 \mathrm{E}-05$ \\
\hline Тe-127 & $2.0 \mathrm{E}-05$ & $1.3 \mathrm{E}-05$ & $\mathrm{Ba}-137 \mathrm{~m}$ & $2.7 \mathrm{E}-09$ & 2.3E-09 \\
\hline Te-127m & $5.9 \mathrm{E}-03$ & $3.9 \mathrm{E}-03$ & Ba-139 & 4.9E-06 & .. $3.5 \mathrm{E}-06$ \\
\hline Te-129 & $3.4 \mathrm{E}-06$ & $2.5 \mathrm{E}-06$ & Ba-140 & $2.5 \mathrm{E}-03$ & $1.6 \mathrm{E}-03$ \\
\hline $\mathrm{Te}-129 \mathrm{~m}$ & $5.8 \mathrm{E}-03$ & $3.8 \mathrm{E}-03$ & Ba-141 & $2.8 \mathrm{E}-06$ & $2.4 \mathrm{E}-06$ \\
\hline $\mathrm{Te}-131$ & $2.9 \mathrm{E}-06$ & $2.4 \mathrm{E}-06$ & Ba-142 & $1.2 \mathrm{E}-06$ & $1.1 \mathrm{E}-06$ \\
\hline Te-131m & $5.5 \mathrm{E}-04$ & $3.6 \mathrm{E}-04$ & La-140 & 9.3E-04 & $6.1 \mathrm{E}-04$ \\
\hline Te-132 & $9.5 \mathrm{E}-04$ & $6.3 \mathrm{E}-04$ & La-141 & $1.6 \mathrm{E}-06$ & $1.1 \mathrm{E}-06$ \\
\hline Te-133 & $1.7 \mathrm{E}-06$ & $1.4 \mathrm{E}-06$ & La-142 & $5.6 \mathrm{E}-05$ & $3.9 \mathrm{E}-05$ \\
\hline Te-133m & $2.6 \mathrm{E}-05$ & $1.9 \mathrm{E}-05$ & Ce-141 & $1.8 \mathrm{E}-03$ & $1.2 \mathrm{E}-03$ \\
\hline Te-134 & 7.3E-06 & $5.7 \mathrm{E}-06$ & $\mathrm{Ce}-143$ & $2.8 \mathrm{E}-04$ & $1.8 \mathrm{E}-04$ \\
\hline I-122 & $5.5 \mathrm{E}-09$ & $1.1 \mathrm{E}-08$ & Ce-144 & $2.9 \mathrm{E}-02$ & $1.9 \mathrm{E}-02$ \\
\hline $\mathrm{I}-123$ & $1.3 \mathrm{E}-05$ & $3.9 \mathrm{E}-05$ & Pr-143 & $1.4 \mathrm{E}-03$ & $9.1 \mathrm{E}-04$ \\
\hline $\mathrm{I}-125$ & $2.4 \mathrm{E}-02$ & 7.0E-02 & $\operatorname{Pr}-144$ & $4.8 \mathrm{E}-07$ & 4.1E-07 \\
\hline
\end{tabular}


Table 4-5. 100-F Area--CAP88-PC Unit Dose Factors by Effective Release Height ${ }^{\mathrm{a}}$ (mrem/Ci).

\begin{tabular}{|c|c|c|c|c|c|}
\hline \multirow{2}{*}{ Nuclide } & \multicolumn{2}{|c|}{$\begin{array}{c}\text { Effective } \\
\text { Release Height }\end{array}$} & \multirow{2}{*}{ Nuclide } & \multicolumn{2}{|c|}{$\begin{array}{c}\text { Effective } \\
\text { Release Height }\end{array}$} \\
\hline & $\mathrm{H}_{\mathrm{eff}}<40 \mathrm{~m}$ & $\mathrm{H}_{\text {eff }} \geq 40 \mathrm{~m}$ & & $\mathrm{H}_{\mathrm{eff}}<40 \mathrm{~m}$ & $\mathrm{H}_{\text {eff } ₹} 40 \mathrm{~m}$ \\
\hline Pr-144m & $3.5 \mathrm{E}-08$ & $2.9 \mathrm{E}-08$ & Po- $211^{c}$ & 0.0 & 0.0 \\
\hline Nd-147 & $1.3 \mathrm{E}-03$ & $8.9 \mathrm{E}-04$ & Po- $212^{c}$ & 0.0 & 0.0 \\
\hline $\mathrm{Pm}-147$ & $2.5 \mathrm{E}-03$ & $1.6 \mathrm{E}-03$ & Po- $213^{c}$ & 0.0 & 0.0 \\
\hline Pm-148 & $2.0 \mathrm{E}-03$ & $1.3 \mathrm{E}-03$ & Po-214 $4^{c}$ & 0.0 & 0.0 \\
\hline $\mathrm{Pm}-148 \mathrm{~m}$ & $1.8 \mathrm{E}-02$ & $1.2 \mathrm{E}-02$ & Po- $215^{\mathrm{c}}$ & 0.0 & 0.0 \\
\hline Pm-149 & $2.5 \mathrm{E}-04$ & $1.6 \mathrm{E}-04$ & Po- $216^{\mathrm{C}}$ & 0.0 & 0.0 \\
\hline $\mathrm{Pm}-151$ & $6.7 \mathrm{E}-05$ & $4.5 \mathrm{E}-05$ & Po-218 & $1.4 \mathrm{E}-09$ & $1.1 \mathrm{E}-09$ \\
\hline $\mathrm{Sm}-147$ & $3.9 E+00$ & $2.5 E+00$ & At $-217^{\mathrm{c}}$ & 0.0 & 0.0 \\
\hline $\mathrm{Sm}-151$ & $1.7 \mathrm{E}-03$ & $1.1 \mathrm{E}-03$ & $\mathrm{Rn}-219^{\mathrm{d}}$ & $(\mathrm{Pb}-211)$ & $(\mathrm{Pb}-211)$ \\
\hline Sm-153 & $1.8 \mathrm{E}-04$ & $1.2 \mathrm{E}-04$ & $\mathrm{Rn}-220^{\mathrm{d}}$ & $(\mathrm{Pb}-212)$ & $(\mathrm{Pb}-212)$ \\
\hline Eu-152 & $5.6 \mathrm{E}-01$ & $3.8 \mathrm{E}-01$ & $R n-222$ & $1.8 \mathrm{E}-04$ & $8.9 \mathrm{E}-05$ \\
\hline $\mathrm{Eu}-152 \mathrm{~m}$ & $2.4 \mathrm{E}-05$ & $1.6 \mathrm{E}-05$ & Fr-221 & $1.1 \mathrm{E}-06$ & $9.0 \mathrm{E}-07$ \\
\hline Eu-154 & $4.5 \mathrm{E}-01$ & $3.0 \mathrm{E}-01$ & Fr-223 & $1.7 \mathrm{E}-05$ & $1.4 \mathrm{E}-05$ \\
\hline Eu-155 & $1.8 \mathrm{E}-02$ & $1.2 \mathrm{E}-02$ & $\mathrm{Ra}-223$ & $5.6 \mathrm{E}-01$ & $3.6 \mathrm{E}-01$ \\
\hline Eu-156 & $5.6 \mathrm{E}-03$ & $3.7 \mathrm{E}-03$ & $\mathrm{Ra}-224$ & $2.0 \mathrm{E}-01$ & $1.3 \mathrm{E}-01$ \\
\hline Gd- $152^{b}$ & (Pu-239) & (Pu-239) & $\mathrm{Ra}-225$ & $3.5 \mathrm{E}-01$ & 2.2E-01 \\
\hline $\mathrm{Tb}-160$ & $1.5 \mathrm{E}-02$ & $9.9 \mathrm{E}-03$ & $\mathrm{Ra}-226$ & $1.1 \mathrm{E}+\infty 0$ & $7.0 \mathrm{E}-01$ \\
\hline Ho-166 & $2.1 \mathrm{E}-04$ & $1.3 \mathrm{E}-04$ & $\mathrm{Ra}-228$ & $4.4 \mathrm{E}-01$ & $2.9 \mathrm{E}-01$ \\
\hline $\mathrm{Ho}-166 \mathrm{~m}$ & $1.8 \mathrm{E}+\infty 0$ & $1.2 \mathrm{E}+00$ & Ac-225 & $3.3 \mathrm{E}-01$ & $2.1 \mathrm{E}-01$ \\
\hline Hf-181 & $5.7 \mathrm{E}-03$ & $3.8 \mathrm{E}-03$ & Ac-227 & $3.4 \mathrm{E}+01$ & $2.2 \mathrm{E}+01$ \\
\hline W-181 & $1.0 \mathrm{E}-03$ & $7.0 \mathrm{E}-04$ & Ac-228 & $3.6 \mathrm{E}-03$ & $2.4 \mathrm{E}-03$ \\
\hline W-185 & $1.4 \mathrm{E}-03$ & $9.3 \mathrm{E}-04$ & Th-227 & $6.1 \mathrm{E}-01$ & $3.9 \mathrm{E}-01$ \\
\hline W-187 & $1.2 \mathrm{E}-04$ & $7.8 \mathrm{E}-05$ & Th-228 & $1.3 \mathrm{E}+01$ & $8.3 E+00$ \\
\hline Re-187 & 4.0E-05 & $2.7 \mathrm{E}-05$ & Th-229 & $3.6 \mathrm{E}+01$ & $2.3 \mathrm{E}+01$ \\
\hline Ir-192 & $1.3 \mathrm{E}-02$ & $8.4 \mathrm{E}-03$ & Th-230 & $1.3 \mathrm{E}+01$ & $8.3 E+00$ \\
\hline $\mathrm{Hg}-203$ & $7.8 \mathrm{E}-03$ & $5.2 \mathrm{E}-03$ & Th-231 & $5.6 \mathrm{E}-05$ & $3.6 \mathrm{E}-05$ \\
\hline Tl-207 & $2.3 \mathrm{E}-09$ & $1.9 \mathrm{E}-09$ & Th-232 & $1.9 \mathrm{E}+01$ & $1.2 \mathrm{E}+01$ \\
\hline Tl-208 & $5.9 \mathrm{E}-08$ & $5.0 \mathrm{E}-08$ & Th-234 & $5.5 \mathrm{E}-03$ & $3.7 \mathrm{E}-03$ \\
\hline Tl-209 & $3.1 \mathrm{E}-09$ & $2.6 \mathrm{E}-09$ & $\mathrm{~Pa}-231$ & $2.7 \mathrm{E}+01$ & $1.7 \mathrm{E}+01$ \\
\hline $\mathrm{Pb}-209$ & $3.5 \mathrm{E}-06$ & $2.4 \mathrm{E}-06$ & $\mathrm{~Pa}-233$ & $2.4 \mathrm{E}-03$ & $1.6 \mathrm{E}-03$ \\
\hline $\mathrm{Pb}-210$ & $3.3 \mathrm{E}+00$ & $2.2 \mathrm{E}+00$ & $\mathrm{~Pa}-234$ & $1.6 \mathrm{E}-04$ & $1.1 \mathrm{E}-04$ \\
\hline $\mathrm{Pb}-211$ & $1.0 \mathrm{E}-04$ & $8.2 \mathrm{E}-05$ & $\mathrm{~Pa}-234 \mathrm{~m}$ & $1.4 \mathrm{E}-14$ & $1.2 \mathrm{E}-14$ \\
\hline $\mathrm{Pb}-212$ & 7.7E-03 & $5.0 \mathrm{E}-03$ & U-232 & $2.6 \mathrm{E}+01$ & $1.6 \mathrm{E}+01$ \\
\hline $\mathrm{Pb}-214$ & $8.7 \mathrm{E}-06$ & $7.2 \mathrm{E}-06$ & U-233 & $7.3 E+00$ & $4.7 \mathrm{E}+00$ \\
\hline $\mathrm{Bi}-210$ & $1.0 \mathrm{E}-02$ & $6.6 \mathrm{E}-03$ & U-234 & $7.2 \mathrm{E}+00$ & $4.6 \mathrm{E}+00$ \\
\hline $\mathrm{Bi}-211$ & $2.0 \mathrm{E}-09$ & $1.7 \mathrm{E}-09$ & $\mathrm{U}-235$ & $6.9 E+00$ & $4.4 \mathrm{E}+\infty 0$ \\
\hline $\mathrm{Bi}-212$ & $6.0 \mathrm{E}-04$ & $4.4 \mathrm{E}-04$ & U-236 & $6.8 \mathrm{E}+00$ & $4.4 \mathrm{E}+\infty 0$ \\
\hline Bi-213 & $1.8 \mathrm{E}-05$ & $1.4 \mathrm{E}-05$ & U-237 & 7.1E-04 & 4.7E-04 \\
\hline Bi-214 & $7.9 \mathrm{E}-06$ & $6.6 \mathrm{E}-06$ & U-238 & $6.4 \mathrm{E}+00$ & $4.1 E+00$ \\
\hline Po-210 & $1.2 \mathrm{E}+00$ & $8.0 \mathrm{E}-01$ & $\mathrm{U}-240$ & $1.2 \mathrm{E}-04$ & $7.7 \mathrm{E}-05$ \\
\hline
\end{tabular}


Table 4-5. 100-F Area--CAP88-PC Unit Dose Factors by Effective Release Height ${ }^{\mathrm{a}}$ (mrem/Ci).

\begin{tabular}{|c|c|c|c|c|c|}
\hline \multirow{2}{*}{ Nuclide } & \multicolumn{2}{|c|}{$\begin{array}{c}\text { Effective } \\
\text { Release Height }\end{array}$} & \multirow{2}{*}{ Nuclide } & \multicolumn{2}{|c|}{$\begin{array}{c}\text { Effective } \\
\text { Release Height }\end{array}$} \\
\hline & $\mathrm{H}_{\mathrm{eff}}<40 \mathrm{~m}$ & $\mathrm{H}_{\mathrm{eff}} \geq 40 \mathrm{~m}$ & & $\mathrm{H}_{\mathrm{eff}}<40 \mathrm{~m}$ & $\mathrm{H}_{\text {eff } \geq} 40 \mathrm{~m}$ \\
\hline $\begin{array}{c}\text { Np-237 } \\
\text { Np-238 } \\
\text { Np-239 } \\
\text { Np-240 } \\
\text { Np-240m } \\
\text { Pu-236 } \\
\text { Pu-238 } \\
\text { Pu-239 } \\
\text { Pu-240 } \\
\text { Pu-241 }\end{array}$ & $\begin{array}{c}.7 \mathrm{E}+01 \\
1.8 \mathrm{E}-03 \\
2.8 \mathrm{E}-04 \\
1.8 \mathrm{E}-05 \\
2.2 \mathrm{E}-07 \\
4.7 \mathrm{E}+00 \\
1.8 \mathrm{E}+01 \\
1.9 \mathrm{E}+01 \\
1.9 \mathrm{E}+01 \\
3.0 \mathrm{E}-01 \\
\end{array}$ & $\begin{array}{c}1.7 \mathrm{E}+01 \\
1.2 \mathrm{E}-03 \\
1.8 \mathrm{E}-04 \\
1.3 \mathrm{E}-05 \\
1.8 \mathrm{E}-07 \\
3.0 \mathrm{E}+00 \\
1.1 \mathrm{E}+01 \\
1.2 \mathrm{E}+01 \\
1.2 \mathrm{E}+01 \\
1.9 \mathrm{E}-01 \\
\end{array}$ & & & \\
\hline $\begin{array}{c}\mathrm{Pu}-242 \\
\mathrm{Pu}-243 \\
\mathrm{Pu}-244 \\
\mathrm{Am}-241 \\
\mathrm{Am}-242 \\
\mathrm{Am}-242 \mathrm{~m} \\
\mathrm{Am}-243 \\
\mathrm{Cm}-242 \\
\mathrm{Cm}-243 \\
\mathrm{Cm}-244\end{array}$ & $\begin{array}{c}1.8 \mathrm{E}+01 \\
1.0 \mathrm{E}-05 \\
1.8 \mathrm{E}+01 \\
2.9 \mathrm{E}+01 \\
2.8 \mathrm{E}-03 \\
2.8 \mathrm{E}+01 \\
2.9 \mathrm{E}+01 \\
9.6 \mathrm{E}-01 \\
2.0 \mathrm{E}+01 \\
1.6 \mathrm{E}+01 \\
\end{array}$ & $\begin{array}{c}1.2 \mathrm{E}+01 \\
6.8 \mathrm{E}-06 \\
1.2 \mathrm{E}+01 \\
1.9 \mathrm{E}+01 \\
1.8 \mathrm{E}-03 \\
1.8 \mathrm{E}+01 \\
1.9 \mathrm{E}+01 \\
6.1 \mathrm{E}-01 \\
1.3 \mathrm{E}+01 \\
9.9 \mathrm{E}+00 \\
\end{array}$ & & & \\
\hline $\begin{array}{c}\mathrm{Cm}-245 \\
\mathrm{Cm}-246 \\
\mathrm{Cm}-247 \\
\mathrm{Cm}-248 \\
\mathrm{Cf}-252\end{array}$ & $\begin{array}{l}3.0 \mathrm{E}+01 \\
3.0 \mathrm{E}+01 \\
2.8 \mathrm{E}+01 \\
1.1 \mathrm{E}+02 \\
8.3 \mathrm{E}+00\end{array}$ & $\begin{array}{l}1.9 \mathrm{E}+01 \\
1.9 \mathrm{E}+01 \\
1.8 \mathrm{E}+01 \\
7.1 \mathrm{E}+01 \\
5.3 \mathrm{E}+00\end{array}$ & & & \\
\hline
\end{tabular}

2 The effective release height, $x$, is in meters.

- CAP88-PC Ver. 1.0 does not calculate doses for Be-10, Se-79, Cd-113, Cd-113m, and Gd-152. Recommend substituting the unit dose factors of the nuclides in parentheses.

c Extremely short lived nuclide, resulting in an offsite dose of zero.

d Short lived radon nuclides only provide the mechanism to circumvent the emission control device. The unit dose factors for the recommended progeny should be used instead. However, 1 ci of Rn does not equal $1 \mathrm{ci}$ of $\mathrm{Pb}$. Multiply the activity of Rn-219 by 0.0018 to get the activity of $\mathrm{Pb}-211$. Multiply the activity of $\mathrm{Rn}-220$ by 0.0014 to get the activity of $\mathrm{Pb}-212$. 
Table 4-6. 100-H Area--CAP88-PC Unit Dose Factors by Effective Release Height ${ }^{\mathrm{a}}$ $(\mathrm{mrem} / \mathrm{Ci})$.

\begin{tabular}{|c|c|c|c|c|c|}
\hline \multirow{2}{*}{ Nuclide } & \multicolumn{2}{|c|}{$\begin{array}{c}\text { Effective } \\
\text { Release Height }\end{array}$} & \multirow{2}{*}{ Nuclide } & \multicolumn{2}{|c|}{$\begin{array}{c}\text { Effective } \\
\text { Release Height }\end{array}$} \\
\hline & $\mathrm{H}_{\text {eff }}<40 \mathrm{~m}$ & $\mathrm{H}_{\text {eff }} \geq 40 \mathrm{~m}$ & & $\mathrm{H}_{\text {eff }}<40 \mathrm{~m}$ & $\mathrm{H}_{\text {eff }} \geq 40 \mathrm{~m}$ \\
\hline $\mathrm{H}-3$ & $5.3 \mathrm{E}-05$ & $2.6 \mathrm{E}-05$ & $\mathrm{Kr}-85$ & $1.2 \mathrm{E}-07$ & $5.8 \mathrm{E}-08$ \\
\hline $\mathrm{Be}-7$ & $3.6 \mathrm{E}-04$ & $2.5 \mathrm{E}-04$ & $\mathrm{Kr}-85 \mathrm{~m}$ & $4.0 \mathrm{E}-06$ & $2.1 \mathrm{E}-06$ \\
\hline $\mathrm{Be}-10^{\mathrm{b}}$ & $(\mathrm{Sr}-90)$ & $(\mathrm{Sr}-90)$ & $\mathrm{Kr}-87$ & $1.1 \mathrm{E}-05$ & $6.2 \mathrm{E}-06$ \\
\hline C-11 & $2.4 \mathrm{E}-06$ & $2.1 \mathrm{E}-06$ & $\mathrm{Kr}-88$ & 4.6E-05 & $2.5 \mathrm{E}-05$ \\
\hline$C-14$ & $4.2 \mathrm{E}-03$ & $2.0 \mathrm{E}-03$ & $\mathrm{Kr}-89$ & $1.2 \mathrm{E}-08$ & $8.5 \mathrm{E}-09$ \\
\hline $\mathrm{C}-15^{\mathrm{c}}$ & 0.0 & 0.0 & $\mathrm{Kr}-90$ & $9.0 \mathrm{E}-24$ & $6.7 \mathrm{E}-24$ \\
\hline $\mathrm{N}-13$ & $6.5 \mathrm{E}-07$ & $5.6 \mathrm{E}-07$ & $\mathrm{Rb}-86$ & $5.2 \mathrm{E}-03$ & $3.6 \mathrm{E}-03$ \\
\hline $0-15$ & $9.4 \mathrm{E}-11$ & $8.1 E-11$ & $R b-87$ & $1.7 \mathrm{E}-02$ & $1.2 \mathrm{E}-02$ \\
\hline F-18 & $1.7 \mathrm{E}-05$ & $1.2 \mathrm{E}-05$ & $\mathrm{Rb}-88$ & $1.7 \mathrm{E}-06$ & $1.4 \mathrm{E}-06$ \\
\hline $\mathrm{Na}-22$ & $2.3 \mathrm{E}-01$ & $1.6 \mathrm{E}-01$ & $\mathrm{Rb}-89$ & $3.5 \mathrm{E}-06$ & $3.0 \mathrm{E}-06$ \\
\hline $\mathrm{Na}-24$ & $3.8 \mathrm{E}-04$ & $2.6 \mathrm{E}-04$ & $\mathrm{Rb}-90$ & $2.7 \mathrm{E}-09$ & $2.3 \mathrm{E}-09$ \\
\hline P-32 & 5.3E-03 & $3.6 \mathrm{E}-03$ & $\mathrm{Rb}-90 \mathrm{~m}$ & $1.1 \mathrm{E}-07$ & $9.4 \mathrm{E}-08$ \\
\hline S-35 & $7.5 \mathrm{E}-04$ & $5.2 \mathrm{E}-04$ & Sr- 89 & $3.1 \mathrm{E}-03$ & $2.2 \mathrm{E}-03$ \\
\hline Ar-41 & 2.1E-05 & $1.2 \mathrm{E}-05$ & Sr -90 & $2.1 \mathrm{E}-01$ & $1.4 \mathrm{E}-01$ \\
\hline $\mathrm{K}-40$ & $2.2 \mathrm{E}-01$ & $1.6 \mathrm{E}-01$ & Sr-91 & $7.8 \mathrm{E}-05$ & $5.3 \mathrm{E}-05$ \\
\hline $\mathrm{Ca}-41$ & $1.9 \mathrm{E}-05$ & $1.3 \mathrm{E}-05$ & Sr-92 & $4.3 \mathrm{E}-05$ & $3.1 \mathrm{E}-05$ \\
\hline Sc-46 & $2.2 \mathrm{E}-02$ & $1.5 \mathrm{E}-02$ & Y-90 & $6.2 \mathrm{E}-04$ & $4.2 \mathrm{E}-04$ \\
\hline $\mathrm{Cr}-51$ & $1.6 \mathrm{E}-04$ & $1.1 \mathrm{E}-04$ & $\mathrm{Y}-90 \mathrm{~m}$ & $1.6 \mathrm{E}-05$ & $1.1 \mathrm{E}-05$ \\
\hline Mn-54 & $3.0 \mathrm{E}-02$ & 2.1E-02 & Y-91 & $4.6 \mathrm{E}-03$ & $3.1 \mathrm{E}-03$ \\
\hline $\mathrm{Mn}-56$ & $4.8 \mathrm{E}-05$ & $3.4 \mathrm{E}-05$ & $\mathrm{Y}-91 \mathrm{~m}$ & $4.0 \mathrm{E}-06$ & $3.1 \mathrm{E}-06$ \\
\hline $\mathrm{Fe}-55$ & $4.2 \mathrm{E}-04$ & $2.9 \mathrm{E}-04$ & Y-92 & $3.9 \mathrm{E}-05$ & $2.7 \mathrm{E}-05$ \\
\hline Fe-59 & $8.6 \mathrm{E}-03$ & $5.9 \mathrm{E}-03$ & Y-93 & $1.0 \mathrm{E}-04$ & $6.9 \mathrm{E}-05$ \\
\hline Co-57 & $5.2 E-03$ & $3.6 \mathrm{E}-03$ & Zr-93 & $2.3 E-03$ & $1.6 E-03$ \\
\hline $\mathrm{Co}-58$ & $9.9 \mathrm{E}-03$ & $6.9 \mathrm{E}-03$ & Zr-95 & 7.1E-03 & $4.9 \mathrm{E}-03$ \\
\hline $\mathrm{Co}-60$ & $4.5 \mathrm{E}-01$ & 3.1E-01 & $\mathrm{Nb}-93 \mathrm{~m}$ & $3.8 \mathrm{E}-03$ & $2.6 \mathrm{E}-03$ \\
\hline Ni-59 & $5.6 \mathrm{E}-04$ & $3.9 \mathrm{E}-04$ & $\mathrm{Nb}-94$ & $1.5 \mathrm{E}+00$ & $1.0 \mathrm{E}+00$ \\
\hline $\mathrm{Ni}-63$ & $4.8 \mathrm{E}-04$ & 3.3E-04 & $\mathrm{Nb}-95$ & $8.7 \mathrm{E}-03$ & $6.0 \mathrm{E}-03$ \\
\hline $\mathrm{Ni}-65$ & $1.9 \mathrm{E}-05$ & $1.3 \mathrm{E}-05$ & $\mathrm{Nb}-95 \mathrm{~m}$ & $5.4 \mathrm{E}-04$ & $3.7 \mathrm{E}-04$ \\
\hline $\mathrm{Cu}-64$ & $2.7 \mathrm{E}-05$ & $1.8 \mathrm{E}-05$ & $\mathrm{Nb}-97$ & 8.7E-06 & $6.6 \mathrm{E}-06$ \\
\hline $\mathrm{Zn}-65$ & $4.5 \mathrm{E}-02$ & $3.1 \mathrm{E}-02$ & $\mathrm{Nb}-97 \mathrm{~m}$ & $8.0 \mathrm{E}-16$ & $6.9 \mathrm{E}-16$ \\
\hline $\mathrm{Zn}-69 \mathrm{~m}$ & $7.3 \mathrm{E}-05$ & $4.9 \mathrm{E}-05$ & Mo-93 & $5.1 \mathrm{E}-03$ & $3.5 \mathrm{E}-03$ \\
\hline $\mathrm{Zn}-69$ & 7.4E-07 & $5.8 \mathrm{E}-07$ & Mo-99 & 3.2E-04 & 2.1E-04 \\
\hline Ga-67 & $1.1 \mathrm{E}-04$ & $7.6 \mathrm{E}-05$ & Tc-97 & $1.0 \mathrm{E}-02$ & $7.2 \mathrm{E}-03$ \\
\hline As-76 & $2.5 \mathrm{E}-04$ & $1.7 \mathrm{E}-04$ & Tc-99 & 4.1E-02 & $2.8 \mathrm{E}-02$ \\
\hline Se-79 & (Pu-241) & (Pu-241) & $\mathrm{Tc}-99 \mathrm{~m}$ & $6.7 \mathrm{E}-06$ & $4.6 \mathrm{E}-06$ \\
\hline $\mathrm{Br}-82$ & $5.8 \mathrm{E}-04$ & $4.0 \mathrm{E}-04$ & Tc-101 & 4.4E-07 & $3.8 \mathrm{E}-07$ \\
\hline $\mathrm{Br}-83$ & $1.4 \mathrm{E}-07$ & $1.0 \mathrm{E}-07$ & Ru-97 & $1.2 \mathrm{E}-04$ & $8.3 \mathrm{E}-05$ \\
\hline $\mathrm{Br}-84$ & $7.9 \mathrm{E}-06$ & $6.6 \mathrm{E}-06$ & $R u-103$ & $3.4 \mathrm{E}-03$ & $2.3 \mathrm{E}-03$ \\
\hline $\mathrm{Br}-85$ & $1.5 \mathrm{E}-10$ & $1.3 \mathrm{E}-10$ & $\mathrm{Ru}-105$ & $4.4 \mathrm{E}-05$ & $3.0 \mathrm{E}-05$ \\
\hline $\mathrm{Kr}-83 \mathrm{~m}$ & $3.7 \mathrm{E}-09$ & 2.1E-09 & Ru-106 & $2.9 \mathrm{E}-02$ & $1.9 \mathrm{E}-02$ \\
\hline
\end{tabular}


Table 4-6. 100-H Area--CAP88-PC Unit Dose Factors by Effective Release Height ${ }^{\mathrm{a}}$. $(\mathrm{mrem} / \mathrm{Ci})$.

\begin{tabular}{|c|c|c|c|c|c|}
\hline \multirow{2}{*}{ Nuclide } & \multicolumn{2}{|c|}{$\begin{array}{c}\text { Effective } \\
\text { Release Height }\end{array}$} & \multirow{2}{*}{ Nuclide } & \multicolumn{2}{|c|}{$\begin{array}{c}\text { Effective } \\
\text { Release Height }\end{array}$} \\
\hline & $\mathrm{H}_{\text {eff }}<40 \mathrm{~m}$ & $\mathrm{H}_{\text {eff }} \geq 40 \mathrm{~m}$ & & $\mathrm{H}_{\mathrm{eff}}<40 \mathrm{~m}$ & $\mathrm{H}_{\text {eff }} \geq 40 \mathrm{~m}$ \\
\hline Rh-103m & $9.4 \mathrm{E}-08$ & $7.3 \mathrm{E}-08$ & I-129 & $2.7 \mathrm{E}-01$ & $8.2 \mathrm{E}-01$ \\
\hline Rh-105 & $8.8 \mathrm{E}-05$ & $5.9 \mathrm{E}-05$ & I-130 & $9.9 \mathrm{E}-05$ & $2.9 \mathrm{E}-04$ \\
\hline $\mathrm{Rh}-105 \mathrm{~m}$ & $1.8 \mathrm{E}-20$ & $1.6 \mathrm{E}-20$ & $\mathrm{I}-131$ & $8.9 \mathrm{E}-03$ & $2.7 \mathrm{E}-02$ \\
\hline $\mathrm{Rh}-106$ & $4.1 \mathrm{E}-26$ & $3.5 \mathrm{E}-26$ & I-132 & $1.5 \mathrm{E}-05$ & $3.9 \mathrm{E}-05$ \\
\hline Pd-107 & $6.8 \mathrm{E}-04$ & 4.6E-04 & I-133 & $1.0 \mathrm{E}-04$ & $3.0 \mathrm{E}-04$ \\
\hline Pd-109 & $6.3 \mathrm{E}-05$ & $4.2 \mathrm{E}-05$ & I-134 & $4.9 \mathrm{E}-06$ & $1.1 \mathrm{E}-05$ \\
\hline $\mathrm{Ag}-109 \mathrm{~m}$ & $5.1 \mathrm{E}-23$ & $4.4 \mathrm{E}-23$ & I-135 & 3.3E-05 & $9.5 \mathrm{E}-05$ \\
\hline $\mathrm{Ag}-110$ & $3.6 \mathrm{E}-32$ & $3.1 \mathrm{E}-32$ & $\mathrm{Xe}-122$ & $2.2 \mathrm{E}-06$ & $1.1 \mathrm{E}-06$ \\
\hline $\mathrm{Ag}-110 \mathrm{~m}$ & $8.8 \mathrm{E}-02$ & $6.1 \mathrm{E}-02$ & $\mathrm{Xe}-123$ & $1.1 \mathrm{E}-05$ & $6.1 \mathrm{E}-06$ \\
\hline$A g-111$ & $1.8 \mathrm{E}-03$ & $1.3 E-03$ & $\mathrm{Xe}-125$ & $8.0 \mathrm{E}-06$ & $4.0 \mathrm{E}-06$ \\
\hline $\mathrm{Cd}-113^{\mathrm{b}}$ & (Pu-241) & (Pu-241) & $\mathrm{Xe}-127$ & $9.2 \mathrm{E}-06$ & $4.5 \mathrm{E}-06$ \\
\hline $\mathrm{Cd}-113 \mathrm{~m}^{\mathrm{b}}$ & (Pu-241) & (Pu-241) & $\mathrm{Xe}-131 \mathrm{~m}$ & $3.4 \mathrm{E}-07$ & $1.7 \mathrm{E}-07$ \\
\hline Cd-115 & $3.5 \mathrm{E}-04$ & $2.4 \mathrm{E}-04$ & $\mathrm{Xe}-133$ & $1.2 \mathrm{E}-06$ & $6.0 \mathrm{E}-07$ \\
\hline $\mathrm{Cd}-115 \mathrm{~m}$ & $6.5 \mathrm{E}-03$ & $4.5 \mathrm{E}-03$ & $\mathrm{Xe}-133 \mathrm{~m}$ & $1.0 \mathrm{E}-06$ & $5.2 \mathrm{E}-07$ \\
\hline In-113m & $4.5 \mathrm{E}-06$ & $3.3 \mathrm{E}-06$ & $\mathrm{Xe}-135$ & $7.3 \mathrm{E}-06$ & $3.7 \mathrm{E}-06$ \\
\hline In-115 & $1.1 \mathrm{E}-01$ & 7.5E-02 & $\mathrm{Xe}-135 \mathrm{~m}$ & $7.6 \mathrm{E}-07$ & $5.7 \mathrm{E}-07$ \\
\hline In-115m & $1.0 \mathrm{E}-05$ & $7.0 \mathrm{E}-06$ & $\mathrm{Xe}-137$ & $3.8 \mathrm{E}-09$ & $2.8 \mathrm{E}-09$ \\
\hline Sn-113 & $4.0 \mathrm{E}-03$ & $2.8 \mathrm{E}-03$ & Xe-138 & $1.9 \mathrm{E}-06$ & 1.4E-06 \\
\hline Sn-123 & $9.4 \mathrm{E}-05$ & $6.5 \mathrm{E}-05$ & Cs-134 & $1.8 \mathrm{E}-01$ & $1.3 \mathrm{E}-01$ \\
\hline Sn-125 & 4.3E-03 & $3.0 \mathrm{E}-03$ & Cs-134m & $1.6 \mathrm{E}-06$ & $1.2 \mathrm{E}-06$ \\
\hline Sn-126 & $8.6 \mathrm{E}-02$ & $5.9 \mathrm{E}-02$ & Cs-135 & $8.0 \mathrm{E}-03$ & $5.6 \mathrm{E}-03$ \\
\hline $\mathrm{Sb}-124$ & $1.6 \mathrm{E}-02$ & $1.1 \mathrm{E}-02$ & Cs- 136 & $6.8 \mathrm{E}-03$ & 4.7E-03 \\
\hline Sb-125 & $4.8 \mathrm{E}-02$ & $3.3 \mathrm{E}-02$ & Cs- 137 & $4.8 \mathrm{E}-02$ & $3.3 \mathrm{E}-02$ \\
\hline $\mathrm{Sb}-126$ & $6.0 \mathrm{E}-03$ & $4.1 \mathrm{E}-03$ & Cs- 138 & $1.1 \mathrm{E}-05$ & $9.1 \mathrm{E}-06$ \\
\hline$S b-126 b$ & $3.5 \mathrm{E}-06$ & $3.0 \mathrm{E}-06$ & Cs-139 & $1.8 E-07$ & $1.5 E-07$ \\
\hline $\mathrm{Sb}-127$ & $8.5 \mathrm{E}-04$ & $5.8 \mathrm{E}-04$ & Ba-133 & $1.4 \mathrm{E}-01$ & $9.4 \mathrm{E}-02$ \\
\hline $\mathrm{Te}-125 \mathrm{~m}$ & $1.9 \mathrm{E}-03$ & $1.3 \mathrm{E}-03$ & Ba-133m & $5.7 \mathrm{E}-05$ & $3.9 \mathrm{E}-05$ \\
\hline Тe-127 & $1.5 \mathrm{E}-05$ & $1.0 \mathrm{E}-05$ & Ba-137m & $5.1 \mathrm{E}-10$ & 4.4E-10 \\
\hline $\mathrm{Te}-127 \mathrm{~m}$ & $4.6 \mathrm{E}-03$ & $3.2 \mathrm{E}-03$ & Ba-139 & $3.4 \mathrm{E}-06$ & $2.5 \mathrm{E}-06$ \\
\hline Te-129 & $2.3 \mathrm{E}-06$ & $1.8 \mathrm{E}-06$ & $\mathrm{Ba}-140$ & $1.9 \mathrm{E}-03$ & $1.3 \mathrm{E}-03$ \\
\hline $\mathrm{Te}-129 \mathrm{~m}$ & $4.6 \mathrm{E}-03$ & $3.1 \mathrm{E}-03$ & Ba-141 & $1.8 \mathrm{E}-06$ & $1.5 \mathrm{E}-06$ \\
\hline Te-131 & $1.8 \mathrm{E}-06$ & $1.6 \mathrm{E}-06$ & Ba-142 & $6.8 \mathrm{E}-07$ & $5.8 \mathrm{E}-07$ \\
\hline $\mathrm{Te}-131 \mathrm{~m}$ & $4.3 \mathrm{E}-04$ & $2.9 \mathrm{E}-04$ & La-140 & $7.3 \mathrm{E}-04$ & $5.0 \mathrm{E}-04$ \\
\hline Te-132 & $7.5 \mathrm{E}-04$ & $5.1 \mathrm{E}-04$ & La-141 & $1.2 \mathrm{E}-06$ & $8.3 \mathrm{E}-07$ \\
\hline Te-133 & 9.6E-07 & $8.3 \mathrm{E}-07$ & La-142 & $3.9 \mathrm{E}-05$ & $2.9 \mathrm{E}-05$ \\
\hline $\mathrm{Te}-133 \mathrm{~m}$ & $1.7 \mathrm{E}-05$ & $1.4 \mathrm{E}-05$ & Ce-141 & $1.4 \mathrm{E}-03$ & $9.4 \mathrm{E}-04$ \\
\hline Te-134 & $4.8 \mathrm{E}-06$ & $3.9 \mathrm{E}-06$ & Ce-143 & 2.2E-04 & $1.4 \mathrm{E}-04$ \\
\hline $\mathrm{l}-122$ & $1.4 \mathrm{E}-09$ & $2.8 \mathrm{E}-09$ & Ce-144 & $2.3 \mathrm{E}-02$ & $1.5 \mathrm{E}-02$ \\
\hline I-123 & $9.1 \mathrm{E}-06$ & $2.7 \mathrm{E}-05$ & Pr-143 & $1.1 \mathrm{E}-03$ & 7.3E-04 \\
\hline $\mathrm{I}-125$ & $1.6 \mathrm{E}-02$ & $4.9 \mathrm{E}-02$ & Pr-144 & $3.0 \mathrm{E}-07$ & $2.6 \mathrm{E}-07$ \\
\hline
\end{tabular}


Table 4-6. 100-H Area--CAP88-PC Unit Dose Factors by Effective Release Height ${ }^{\mathrm{a}}$ (mrem/Ci).

\begin{tabular}{|c|c|c|c|c|c|}
\hline \multirow{2}{*}{ Nuclide } & \multicolumn{2}{|c|}{$\begin{array}{c}\text { Effective } \\
\text { Release Height }\end{array}$} & \multirow{2}{*}{ Nuclide } & \multicolumn{2}{|c|}{$\begin{array}{c}\text { Effective } \\
\text { Release Height }\end{array}$} \\
\hline & $\mathrm{H}_{\mathrm{eff}}<40 \mathrm{~m}$ & $\mathrm{H}_{\text {eff } 240 \mathrm{~m}}$ & & $\mathrm{H}_{\mathrm{eff}}<40 \mathrm{~m}$ & $\mathrm{H}_{\text {eff }} \geq 40 \mathrm{~m}$ \\
\hline $\operatorname{Pr}-144 \mathrm{~m}$ & $1.6 \mathrm{E}-08$ & $1.4 \mathrm{E}-08$ & $P_{0-211^{c}}$ & 0.0 & 0.0 \\
\hline Nd-147 & $1.1 \mathrm{E}-03$ & 7.2E-04 & Po-212 & 0.0 & 0.0 \\
\hline Pm-147 & $2.0 \mathrm{E}-03$ & $1.3 \mathrm{E}-03$ & Po-213 & 0.0 & 0.0 \\
\hline $\mathrm{Pm}-148$ & $1.6 \mathrm{E}-03$ & 1.1E-03 & Po-214 & 0.0 & 0.0 \\
\hline Pm-148m & $1.4 \mathrm{E}-02$ & $9.7 \mathrm{E}-03$ & Po- $215^{\mathrm{C}}$ & 0.0 & 0.0 \\
\hline Pm-149 & $2.0 \mathrm{E}-04$ & $1.3 \mathrm{E}-04$ & Po-216 & 0.0 & 0.0 \\
\hline Pm-151 & $5.2 \mathrm{E}-05$ & $3.6 \mathrm{E}-05$ & Po-218 & $3.2 \mathrm{E}-10$ & $2.7 \mathrm{E}-10$ \\
\hline Sm-147 & $3.1 \mathrm{E}+\infty$ & $2.0 \mathrm{E}+00$ & At $-217^{c}$ & 0.0 & 0.0 \\
\hline Sm-151 & $1.4 \mathrm{E}-03$ & $9.0 \mathrm{E}-04$ & $\mathrm{Rn}-219^{\mathrm{d}}$ & $(\mathrm{Pb}-211)$ & $(\mathrm{Pb}-211)$ \\
\hline $\mathrm{Sm}-153$ & $1.4 \mathrm{E}-04$ & 9.4E-05 & $\mathrm{Rn}=220^{\mathrm{d}}$ & $(\mathrm{Pb}-212)$ & $(\mathrm{Pb}-212)$ \\
\hline Eu-152 & $4.4 \mathrm{E}-01$ & $3.0 \mathrm{E}-01$ & $\mathrm{Rn}-222$ & $1.5 \mathrm{E}-04$ & $7.3 \mathrm{E}-05$ \\
\hline $\mathrm{Eu}-152 \mathrm{~m}$ & $1.8 \mathrm{E}-05$ & $1.2 \mathrm{E}-05$ & Fr-221 & $3.9 \mathrm{E}-07$ & 3.3E-07 \\
\hline Eu-154 & $3.6 \mathrm{E}-01$ & $2.5 \mathrm{E}-01$ & $\mathrm{Fr}-223$ & 1.1E-05 & $9.4 \mathrm{E}-06$ \\
\hline Eu-155 & $1.5 \mathrm{E}-02$ & 1.0E-02 & Ra-223 & $4.4 \mathrm{E}-01$ & $2.9 \mathrm{E}-01$ \\
\hline Eu-156 & $4.4 E-03$ & $3.0 \mathrm{E}-03$ & Ra-224 & $1.6 \mathrm{E}-01$ & $1.0 \mathrm{E}-01$ \\
\hline $\mathrm{Gd}-152^{b}$ & (Pu-239) & (Pu-239) & $\mathrm{Ra}-225$ & 2.7E-01 & $1.8 \mathrm{E}-01$ \\
\hline $\mathrm{Tb}-160$ & $1.2 \mathrm{E}-02$ & $8.0 \mathrm{E}-03$ & $\mathrm{Ra}-226$ & $8.4 \mathrm{E}-01$ & $5.7 \mathrm{E}-01$ \\
\hline Ho-166 & $1.6 \mathrm{E}-04$ & $1.1 \mathrm{E}-04$ & Ra-228 & $3.5 \mathrm{E}-01$ & $2.4 \mathrm{E}-01$ \\
\hline $\mathrm{Ho}-166 \mathrm{~m}$ & $1.4 \mathrm{E}+\infty$ & $1.0 \mathrm{E}+\infty 0$ & Ac-225 & $2.6 \mathrm{E}-01$ & $1.7 \mathrm{E}-01$ \\
\hline Hf-181 & $4.5 \mathrm{E}-03$ & $3.1 \mathrm{E}-03$ & Ac-227 & $2.7 \mathrm{E}+01$ & $1.8 \mathrm{E}+01$ \\
\hline W-181 & $8.2 \mathrm{E}-04$ & $5.7 \mathrm{E}-04$ & Ac- 228 & $2.7 \mathrm{E}-03$ & $1.8 \mathrm{E}-03$ \\
\hline W-185 & $1.1 \mathrm{E}-03$ & $7.5 \mathrm{E}-04$ & Th-227 & $4.8 \mathrm{E}-01$ & $3.1 \mathrm{E}-01$ \\
\hline W-187 & $9.2 \mathrm{E}-05$ & $6.3 \mathrm{E}-05$ & Th-228 & $1.0 \mathrm{E}+01$ & $6.7 \mathrm{E}+00$ \\
\hline $\operatorname{Re}-187$ & $3.1 \mathrm{E}-05$ & $2.2 \mathrm{E}-05$ & Th-229 & $2.8 \mathrm{E}+01$ & $1.9 \mathrm{E}+01$ \\
\hline Ir-192 & $9.9 \mathrm{E}-03$ & $6.8 \mathrm{E}-03$ & Th-230 & $1.0 \mathrm{E}+01$ & $6.7 \mathrm{E}+\infty 0$ \\
\hline $\mathrm{Hg}-203$ & $6.2 \mathrm{E}-03$ & $4.3 E-03$ & Th-231 & $4.3 \mathrm{E}-05$ & $2.9 \mathrm{E}-05$ \\
\hline T1-207 & $8.2 \mathrm{E}-10$ & $7.0 \mathrm{E}-10$ & Th-232 & $1.5 \mathrm{E}+01$ & $9.6 \mathrm{E}+\infty$ \\
\hline Tl-208 & $1.4 \mathrm{E}-08$ & $1.2 \mathrm{E}-08$ & Th-234 & $4.4 \mathrm{E}-03$ & $3.0 \mathrm{E}-03$ \\
\hline Tl-209 & $4.6 \mathrm{E}-10$ & $3.9 \mathrm{E}-10$ & $\mathrm{~Pa}-231$ & $2.1 \mathrm{E}+01$ & $1.4 \mathrm{E}+01$ \\
\hline $\mathrm{Pb}-209$ & $2.6 \mathrm{E}-06$ & $1.8 \mathrm{E}-06$ & $\mathrm{~Pa}-233$ & $1.9 \mathrm{E}-03$ & $1.3 \mathrm{E}-03$ \\
\hline $\mathrm{Pb}-210$ & $2.6 \mathrm{E}+00$ & $1.8 \mathrm{E}+00$ & $\mathrm{~Pa}-234$ & $1.2 \mathrm{E}-04$ & $8.3 \mathrm{E}-05$ \\
\hline $\mathrm{Pb}-211$ & $6.8 \mathrm{E}-05$ & $5.6 \mathrm{E}-05$ & $\mathrm{~Pa}-234 \mathrm{~m}$ & $5.0 \mathrm{E}-16$ & $4.2 \mathrm{E}-16$ \\
\hline $\mathrm{Pb}-212$ & $5.9 \mathrm{E}-03$ & $3.9 \mathrm{E}-03$ & U-232 & $2.0 \mathrm{E}+01$ & $1.3 E+01$ \\
\hline $\mathrm{Pb}-214$ & $5.6 \mathrm{E}-06$ & $4.8 \mathrm{E}-06$ & U-233 & $5.7 \mathrm{E}+\infty$ & $3.8 \mathrm{E}+00$ \\
\hline $\mathrm{Bi}-210$ & $8.1 E-03$ & $5.4 \mathrm{E}-03$ & U-234 & $5.7 \mathrm{E}+\infty 0$ & $3.7 \mathrm{E}+00$ \\
\hline $\mathrm{Bi}-211$ & $2.8 \mathrm{E}-10$ & $2.4 \mathrm{E}-10$ & U-235 & $5.4 \mathrm{E}+\infty 0$ & $3.6 \mathrm{E}+00$ \\
\hline $\mathrm{Bi}-212$ & $4.0 \mathrm{E}-04$ & $3.1 \mathrm{E}-04$ & U-236 & $5.4 \mathrm{E}+\infty 0$ & $3.5 E+00$ \\
\hline $\mathrm{Bi}-213$ & $1.2 \mathrm{E}-05$ & $9.6 \mathrm{E}-06$ & U-237 & $5.6 \mathrm{E}-04$ & $3.8 \mathrm{E}-04$ \\
\hline Bi-214 & $4.9 \mathrm{E}-06$ & 4.2E-06 & U-238 & $5.0 \mathrm{E}+00$ & $3.3 E+00$ \\
\hline Po-210 & $9.5 \mathrm{E}-01$ & $6.5 \mathrm{E}-01$ & $\mathrm{U}-240$ & 9.2E-05 & $6.2 \mathrm{E}-05$ \\
\hline
\end{tabular}


Table 4-6. 100-H Area--CAP88-PC Unit Dose Factors by Effective Release Height ${ }^{\mathrm{a}}$ $(\mathrm{mrem} / \mathrm{Ci})$.

\begin{tabular}{|c|c|c|l|c|c|}
\hline \multirow{2}{*}{ Nuclide } & \multicolumn{2}{|c|}{ Release Height } & \multirow{2}{*}{ Nuclide } & \multicolumn{2}{c|}{$\begin{array}{c}\text { Effective } \\
\text { Release Height }\end{array}$} \\
\cline { 2 - 3 } & $\mathrm{H}_{\text {eff }}<40 \mathrm{~m}$ & $\mathrm{H}_{\text {eff }} \geq 40 \mathrm{~m}$ & & $\mathrm{H}_{\text {eff }}<40 \mathrm{~m}$ & $\mathrm{H}_{\text {eff }} \geq 40 \mathrm{~m}$ \\
\hline $\mathrm{Np}-237$ & $2.1 \mathrm{E}+01$ & $1.4 \mathrm{E}+01$ & & & \\
Np-238 & $1.4 \mathrm{E}-03$ & $9.5 \mathrm{E}-04$ & & & \\
$\mathrm{~Np}-239$ & $2.2 \mathrm{E}-04$ & $1.5 \mathrm{E}-04$ & & & \\
$\mathrm{~Np}-240$ & $1.2 \mathrm{E}-05$ & $9.3 \mathrm{E}-06$ & & & \\
$\mathrm{~Np}-240 \mathrm{~m}$ & $1.0 \mathrm{E}-07$ & $8.7 \mathrm{E}-08$ & & & \\
$\mathrm{Pu}-236$ & $3.7 \mathrm{E}+00$ & $2.4 \mathrm{E}+00$ & & & \\
$\mathrm{Pu}-238$ & $1.4 \mathrm{E}+01$ & $9.2 \mathrm{E}+00$ & & & \\
$\mathrm{Pu}-239$ & $1.5 \mathrm{E}+01$ & $9.9 \mathrm{E}+00$ & & & \\
$\mathrm{Pu}-240$ & $1.5 \mathrm{E}+01$ & $9.9 \mathrm{E}+00$ & & & \\
$\mathrm{Pu}-241$ & $2.4 \mathrm{E}-01$ & $1.6 \mathrm{E}-01$ & & & \\
\hline $\mathrm{Pu}-242$ & $1.4 \mathrm{E}+01$ & $9.5 \mathrm{E}+00$ & & & \\
$\mathrm{Pu}-243$ & $7.7 \mathrm{E}-06$ & $5.2 \mathrm{E}-06$ & & & \\
$\mathrm{Pu}-244$ & $1.4 \mathrm{E}+01$ & $9.4 \mathrm{E}+00$ & & & \\
$\mathrm{Am}-241$ & $2.3 \mathrm{E}+01$ & $1.5 \mathrm{E}+01$ & & & \\
$\mathrm{Am}-242$ & $2.2 \mathrm{E}-03$ & $1.5 \mathrm{E}-03$ & & & \\
$\mathrm{Am}-242 \mathrm{~m}$ & $2.2 \mathrm{E}+01$ & $1.5 \mathrm{E}+01$ & & & \\
$\mathrm{Am}-243$ & $2.3 \mathrm{E}+01$ & $1.5 \mathrm{E}+01$ & & & \\
$\mathrm{Cm}-242$ & $7.5 \mathrm{E}-01$ & $5.0 \mathrm{E}-01$ & & & \\
$\mathrm{Cm}-243$ & $1.6 \mathrm{E}+01$ & $1.0 \mathrm{E}+01$ & & & \\
$\mathrm{Cm}-244$ & $1.2 \mathrm{E}+01$ & $8.0 \mathrm{E}+00$ & & & \\
\hline $\mathrm{Cm}-245$ & $2.4 \mathrm{E}+01$ & $1.6 \mathrm{E}+01$ & & & \\
$\mathrm{Cm}-246$ & $2.4 \mathrm{E}+01$ & $1.6 \mathrm{E}+01$ & & & \\
$\mathrm{Cm}-247$ & $2.2 \mathrm{E}+01$ & $1.5 \mathrm{E}+01$ & & & \\
$\mathrm{Cm}-248$ & $8.7 \mathrm{E}+01$ & $5.7 \mathrm{E}+01$ & & & \\
$\mathrm{Cf}-252$ & $6.5 \mathrm{E}+00$ & $4.3 \mathrm{E}+00$ & & & \\
\hline
\end{tabular}

2 The effective release height, $\mathrm{x}$, is in meters.

b CAP88-PC Ver. 1.0 does not calculate doses for Be-10, Se-79, Cd-113, Cd-113m, and Gd-152. Recommend substituting the unit dose factors of the nuclides in parentheses.

- Extremely short lived nuclide, resulting in an offsite dose of zero.

d Short lived radon nuclides only provide the mechanism to circumvent the emission control device. The unit dose factors for the recommended progeny should be used instead. However, 1 ci of $\mathrm{Rn}$ does not equal 1 ci of $\mathrm{Pb}$. Multiply the activity of Rn-219 by 0.0018 to get the activity of $\mathrm{Pb}-211$. Multiply the activity of $\mathrm{Rn}-220$ by 0.0014 to get the activity of $\mathrm{Pb}-212$. 
Table 4-7. 100-K Area--CAP88-PC Unit Dose Factors by Effective Release Height ${ }^{\mathrm{a}}$ (mrem/Ci).

\begin{tabular}{|c|c|c|c|c|c|}
\hline \multirow{2}{*}{ Nuclide } & \multicolumn{2}{|c|}{$\begin{array}{c}\text { Effective } \\
\text { Release Height }\end{array}$} & \multirow{2}{*}{ Nuclide } & \multicolumn{2}{|c|}{$\begin{array}{c}\text { Effective } \\
\text { Release Height }\end{array}$} \\
\hline & $\mathrm{H}_{\mathrm{eff}}<40 \mathrm{~m}$ & $\mathrm{H}_{\text {eff }} \geq 40 \mathrm{~m}$ & & $\mathrm{H}_{\mathrm{eff}}<40 \mathrm{~m}$ & $\mathrm{H}_{\text {eff }} \geq 40 \mathrm{~m}$ \\
\hline $\mathrm{H}-3$ & $4.9 \mathrm{E}-05$ & $2.5 \mathrm{E}-05$ & $\mathrm{Kr}-85$ & $1.1 \mathrm{E}-07$ & 5.6E-08 \\
\hline $\mathrm{Be}-7$ & $3.9 \mathrm{E}-04$ & $2.4 \mathrm{E}-04$ & $\mathrm{Kr}-85 \mathrm{~m}$ & $4.2 \mathrm{E}-06$ & $2.2 \mathrm{E}-06$ \\
\hline $\mathrm{Be}-10^{\mathrm{b}}$ & $(\mathrm{Sr}-90)$ & $(\mathrm{Sr}-90)$ & $\mathrm{Kr}-87$ & $1.5 \mathrm{E}-05$ & 7.3E-06 \\
\hline C-11 & $3.6 \mathrm{E}-06$ & $2.1 \mathrm{E}-06$ & $\mathrm{Kr}-88$ & $5.3 \mathrm{E}-05$ & 2.7E-05 \\
\hline C-14 & $3.8 \mathrm{E}-03$ & $2.0 \mathrm{E}-03$ & $\mathrm{Kr}-89$ & $3.6 \mathrm{E}-08$ & $1.9 \mathrm{E}-08$ \\
\hline $\mathrm{C}-15^{\mathrm{c}}$ & 0.0 & 0.0 & Kr-90 & $8.3 \mathrm{E}-20$ & $4.3 \mathrm{E}-20$ \\
\hline $\mathrm{N}-13$ & $8.3 \mathrm{E}-07$ & $4.9 \mathrm{E}-07$ & $\mathrm{Rb}-86$ & $5.7 E-03$ & $3.6 \mathrm{E}-03$ \\
\hline $0-15$ & 7.3E-10 & $4.4 \mathrm{E}-10$ & Rb-87 & $1.9 \mathrm{E}-02$ & $1.2 \mathrm{E}-02$ \\
\hline F-18 & $2.3 \mathrm{E}-05$ & $1.4 \mathrm{E}-05$ & $\mathrm{Rb}-88$ & $2.4 \mathrm{E}-06$ & $1.4 \mathrm{E}-06$ \\
\hline $\mathrm{Na}-22$ & $2.5 \mathrm{E}-01$ & $1.6 \mathrm{E}-01$ & $\mathrm{Rb}-89$ & $4.8 \mathrm{E}-06$ & $2.8 \mathrm{E}-06$ \\
\hline $\mathrm{Na}-24$ & $4.3 \mathrm{E}-04$ & $2.7 \mathrm{E}-04$ & Rb-90 & $1.2 \mathrm{E}-08$ & $7.1 \mathrm{E}-09$ \\
\hline P-32 & $5.7 \mathrm{E}-03$ & $3.6 \mathrm{E}-03$ & Rb-90m & $2.3 \mathrm{E}-07$ & $1.4 \mathrm{E}-07$ \\
\hline S-35 & 8.1E-04 & $5.1 \mathrm{E}-04$ & Sr-89 & $3.4 \mathrm{E}-03$ & $2.1 \mathrm{E}-03$ \\
\hline $\operatorname{Ar}-41$ & $2.6 \mathrm{E}-05$ & $1.3 \mathrm{E}-05$ & Sr-90 & $2.2 \mathrm{E}-01$ & $1.4 \mathrm{E}-01$ \\
\hline $\mathrm{K}-40$ & $2.4 \mathrm{E}-01$ & $1.5 \mathrm{E}-01$ & Sr-91 & 9.0E-05 & $5.5 \mathrm{E}-05$ \\
\hline $\mathrm{Ca}-41$ & $2.0 \mathrm{E}-05$ & $1.3 \mathrm{E}-05$ & Sr-92 & $5.7 \mathrm{E}-05$ & $3.4 \mathrm{E}-05$ \\
\hline Sc-46 & $2.4 \mathrm{E}-02$ & $1.5 \mathrm{E}-02$ & Y.90 & $6.8 \mathrm{E}-04$ & $4.2 \mathrm{E}-04$ \\
\hline Cr-51 & $1.7 \mathrm{E}-04$ & $1.1 \mathrm{E}-04$ & $\mathrm{Y}-90 \mathrm{~m}$ & $2.0 \mathrm{E}-05$ & $1.2 \mathrm{E}-05$ \\
\hline Mn-54 & $3.3 \mathrm{E}-02$ & $2.1 \mathrm{E}-02$ & Y-91 & $5.0 \mathrm{E}-03$ & $3.1 \mathrm{E}-03$ \\
\hline $\mathrm{Mn}-56$ & $6.4 \mathrm{E}-05$ & $3.8 \mathrm{E}-05$ & Y-91m & $6.4 \mathrm{E}-06$ & $3.8 \mathrm{E}-06$ \\
\hline Fe-55 & $4.5 \mathrm{E}-04$ & $2.9 \mathrm{E}-04$ & Y-92 & $4.9 \mathrm{E}-05$ & $2.9 \mathrm{E}-05$ \\
\hline Fe-59 & $9.3 \mathrm{E}-03$ & $5.9 \mathrm{E}-03$ & Y-93 & $1.2 \mathrm{E}-04$ & 7.2E-05 \\
\hline Co-57 & $5.6 \mathrm{E}-03$ & $3.5 \mathrm{E}-03$ & Zr-93 & $2.6 \mathrm{E}-03$ & $1.6 \mathrm{E}-03$ \\
\hline Co-58 & $1.1 \mathrm{E}-02$ & $6.8 \mathrm{E}-03$ & Zr-95 & $7.7 \mathrm{E}-03$ & $4.8 \mathrm{E}-03$ \\
\hline Co- 60 & $4.9 \mathrm{E}-01$ & $3.1 \mathrm{E}-01$ & $\mathrm{Nb}-93 \mathrm{~m}$ & $4.1 \mathrm{E}-03$ & $2.5 \mathrm{E}-03$ \\
\hline Ni-59 & $6.1 \mathrm{E}-04$ & $3.8 \mathrm{E}-04$ & $\mathrm{Nb}-94$ & $1.6 \mathrm{E}+00$ & $1.0 \mathrm{E}+00$ \\
\hline $\mathrm{Ni}-63$ & $5.2 \mathrm{E}-04$ & $3.2 \mathrm{E}-04$ & $\mathrm{Nb}-95$ & $9.5 \mathrm{E}-03$ & $6.0 \mathrm{E}-03$ \\
\hline $\mathrm{Ni}-65$ & $2.5 \mathrm{E}-05$ & $1.5 \mathrm{E}-05$ & $\mathrm{Nb}-95 \mathrm{~m}$ & $5.9 \mathrm{E}-04$ & $3.7 \mathrm{E}-04$ \\
\hline $\mathrm{Cu}-64$ & $3.1 \mathrm{E}-05$ & $1.9 \mathrm{E}-05$ & $\mathrm{Nb}-97$ & $1.3 \mathrm{E}-05$ & $7.9 \mathrm{E}-06$ \\
\hline $\mathrm{Zn}-65$ & $4.9 \mathrm{E}-02$ & $3.1 \mathrm{E}-02$ & $\mathrm{Nb}-97 \mathrm{~m}$ & $8.7 \mathrm{E}-14$ & 5.3E-14 \\
\hline $\mathrm{Zn}-69 \mathrm{~m}$ & $8.3 \mathrm{E}-05$ & 5.1E-05 & Mo-93 & $5.6 \mathrm{E}-03$ & $3.5 \mathrm{E}-03$ \\
\hline $\mathrm{Zn}-69$ & $1.2 \mathrm{E}-06$ & $7.0 \mathrm{E}-07$ & Mo-99 & $3.5 \mathrm{E}-04$ & $2.1 \mathrm{E}-04$ \\
\hline Ga-67 & $1.2 \mathrm{E}-04$ & $7.6 \mathrm{E}-05$ & Tc-97 & $1.1 \mathrm{E}-02$ & $7.2 \mathrm{E}-03$ \\
\hline As-76 & $2.7 \mathrm{E}-04$ & $1.7 \mathrm{E}-04$ & Tc-99 & $4.5 \mathrm{E}-02$ & $2.8 \mathrm{E}-02$ \\
\hline $\mathrm{Se}-79^{\mathrm{b}}$ & (Pu-241) & (Pu-241) & Tc-99m & $7.9 \mathrm{E}-06$ & $4.9 \mathrm{E}-06$ \\
\hline $\mathrm{Br}-82$ & $6.4 \mathrm{E}-04$ & $4.0 \mathrm{E}-04$ & Tc-101 & $6.0 \mathrm{E}-07$ & $3.5 \mathrm{E}-07$ \\
\hline Br-83 & $1.9 \mathrm{E}-07$ & $1.2 \mathrm{E}-07$ & Ru-97 & $1.3 \mathrm{E}-04$ & 8.2E-05 \\
\hline Br-84 & $1.3 \mathrm{E}-05$ & $7.6 \mathrm{E}-06$ & $\mathrm{Ru}-103$ & $3.6 \mathrm{E}-03$ & $2.3 \mathrm{E}-03$ \\
\hline Br-85 & 5.7E-10 & $3.4 \mathrm{E}-10$ & Ru-105 & $5.4 \mathrm{E}-05$ & 3.3E-05 \\
\hline $\mathrm{Kr}-83 \mathrm{~m}$ & $4.7 \mathrm{E}-09$ & 2.4E-09 & $\mathrm{Ru}-106$ & $3.1 \mathrm{E}-02$ & $1.9 \mathrm{E}-02$ \\
\hline
\end{tabular}


HNF-3602, Vol. 1

Table 4-7. 100-K Area--CAP88-PC Unit Dose Factors by Effective Release Height ${ }^{a}$ $(\mathrm{mrem} / \mathrm{Ci})$.

\begin{tabular}{|c|c|c|c|c|c|}
\hline \multirow{2}{*}{ Nuclide } & \multicolumn{2}{|c|}{$\begin{array}{c}\text { Effective } \\
\text { Release Height }\end{array}$} & \multirow{2}{*}{ Nuclide } & \multicolumn{2}{|c|}{$\begin{array}{c}\text { Effective } \\
\text { Release Height }\end{array}$} \\
\hline & $\mathrm{H}_{\mathrm{eff}}<40 \mathrm{~m}$ & $\mathrm{H}_{\text {eff }} \geq 40 \mathrm{~m}$ & & $\mathrm{H}_{\mathrm{eff}}<40 \mathrm{~m}$ & $\mathrm{H}_{\text {eff }} \geq 40 \mathrm{~m}$ \\
\hline $\mathrm{Rh}-103 \mathrm{~m}$ & $1.5 \mathrm{E}-07$ & $8.9 \mathrm{E}-08$ & I-129 & $2.4 \mathrm{E}-01$ & $1.1 \mathrm{E}+00$ \\
\hline $\mathrm{Rh}-105$ & $9.7 \mathrm{E}-05$ & $5.9 \mathrm{E}-05$ & $\mathrm{I}-130$ & $8.9 \mathrm{E}-05$ & $3.9 \mathrm{E}-04$ \\
\hline Rh-105m & $1.1 \mathrm{E}-17$ & $6.8 \mathrm{E}-18$ & $\mathrm{I}-131$ & $8.0 \mathrm{E}-03$ & $3.5 \mathrm{E}-02$ \\
\hline $\mathrm{Rh}-106$ & $8.2 \mathrm{E}-22$ & $5.0 \mathrm{E}-22$ & $\mathrm{I}-132$ & $1.3 \mathrm{E}-05$ & $5.7 \mathrm{E}-05$ \\
\hline Pd-107 & 7.4E-04 & 4.5E-04 & I-133 & $9.2 \mathrm{E}-05$ & $4.0 \mathrm{E}-04$ \\
\hline Pd-109 & 7.2E-05 & 4.3E-05 & $\mathrm{I}-134$ & $3.6 \mathrm{E}-06$ & $1.6 \mathrm{E}-05$ \\
\hline $\mathrm{Ag}-109 \mathrm{~m}$ & $8.0 \mathrm{E}-20$ & $4.9 \mathrm{E}-20$ & I-135 & $2.9 \mathrm{E}-05$ & $1.3 \mathrm{E}-04$ \\
\hline Ag-110 & 7.4E-26 & $4.5 \mathrm{E}-26$ & $\mathrm{Xe}-122$ & 2.1E-06 & 1.1E-06 \\
\hline $\mathrm{Ag}-110 \mathrm{~m}$ & $9.5 \mathrm{E}-02$ & $6.0 \mathrm{E}-02$ & $\mathrm{Xe}-123$ & $1.3 \mathrm{E}-05$ & $6.8 \mathrm{E}-06$ \\
\hline $\mathrm{Ag}-111$ & $2.0 \mathrm{E}-03$ & $1.3 \mathrm{E}-03$ & $\mathrm{Xe}-125$ & 7.6E-06 & $3.9 \mathrm{E}-06$ \\
\hline $\mathrm{Cd}-113^{\mathrm{b}}$ & (Pu-241) & (Pu-241) & $\mathrm{Xe}-127$ & $8.5 \mathrm{E}-06$ & $4.4 \mathrm{E}-06$ \\
\hline $\mathrm{Cd}-113 \mathrm{~m}^{\mathrm{b}}$ & (Pu-241) & (Pu-241) & $\mathrm{Xe}-131 \mathrm{~m}$ & $3.1 \mathrm{E}-07$ & $1.6 \mathrm{E}-07$ \\
\hline Cd-115 & $3.8 \mathrm{E}-04$ & $2.4 \mathrm{E}-04$ & $\mathrm{Xe}-133$ & $1.1 \mathrm{E}-06$ & $5.8 \mathrm{E}-07$ \\
\hline $\mathrm{Cd}-115 \mathrm{~m}$ & $7.1 \mathrm{E}-03$ & 4.4E-03 & $\mathrm{Xe}-133 \mathrm{~m}$ & $9.7 \mathrm{E}-07$ & $5.0 \mathrm{E}-07$ \\
\hline In-113m & $6.5 \mathrm{E}-06$ & $3.9 \mathrm{E}-06$ & Xe-135 & $7.2 \mathrm{E}-06$ & $3.7 \mathrm{E}-06$ \\
\hline In-115 & $1.2 \mathrm{E}-01$ & 7.4E-02 & $\mathrm{Xe}-135 \mathrm{~m}$ & $1.1 \mathrm{E}-06$ & $5.2 \mathrm{E}-07$ \\
\hline In-115m & $1.3 \mathrm{E}-05$ & $7.6 \mathrm{E}-06$ & $\mathrm{Xe}-137$ & $9.0 \mathrm{E}-09$ & 4.7E-09 \\
\hline Sn-113 & $4.4 \mathrm{E}-03$ & $2.8 \mathrm{E}-03$ & Xe-138 & $2.6 \mathrm{E}-06$ & $1.3 \mathrm{E}-06$ \\
\hline Sn-123 & $1.0 \mathrm{E}-04$ & $6.4 \mathrm{E}-05$ & Cs-134 & $2.0 \mathrm{E}-01$ & $1.3 \mathrm{E}-01$ \\
\hline $\mathrm{Sn}-125$ & $4.7 \mathrm{E}-03$ & $3.0 \mathrm{E}-03$ & Cs $-134 m$ & 2.1E-06 & 1.3E-06 \\
\hline Sn-126 & $9.3 \mathrm{E}-02$ & $5.9 \mathrm{E}-02$ & Cs-135 & $8.7 \mathrm{E}-03$ & $5.5 \mathrm{E}-03$ \\
\hline Sb-124 & $1.7 \mathrm{E}-02$ & $1.1 \mathrm{E}-02$ & Cs-136 & 7.3E-03 & $4.6 \mathrm{E}-03$ \\
\hline $\mathrm{Sb}-125$ & $5.2 \mathrm{E}-02$ & $3.3 \mathrm{E}-02$ & Cs-137 & $5.3 \mathrm{E}-02$ & $3.3 \mathrm{E}-02$ \\
\hline Sb-126 & $6.5 \mathrm{E}-03$ & $4.1 \mathrm{E}-03$ & Cs-138 & $1.8 \mathrm{E}-05$ & $1.0 \mathrm{E}-05$ \\
\hline Sb-126b & $5.1 \mathrm{E}-06$ & $2.9 \mathrm{E}-06$ & Cs-139 & $2.3 \mathrm{E}-07$ & $1.3 \mathrm{E}-07$ \\
\hline Sb-127 & $9.3 \mathrm{E}-04$ & $5.8 \mathrm{E}-04$ & Ba-133 & $1.5 \mathrm{E}-01$ & $9.3 \mathrm{E}-02$ \\
\hline $\mathrm{Te}-125 \mathrm{~m}$ & $2.0 \mathrm{E}-03$ & $1.3 \mathrm{E}-03$ & Ba-133m & $6.3 \mathrm{E}-05$ & $3.9 \mathrm{E}-05$ \\
\hline Te-127 & $1.7 \mathrm{E}-05$ & $1.0 \mathrm{E}-05$ & $\mathrm{Ba}-137 \mathrm{~m}$ & $2.4 \mathrm{E}-09$ & $1.4 \mathrm{E}-09$ \\
\hline $\mathrm{Te}-127 \mathrm{~m}$ & $5.0 \mathrm{E}-03$ & $3.1 \mathrm{E}-03$ & $\mathrm{Ba}-139$ & $5.1 \mathrm{E}-06$ & $3.0 \mathrm{E}-06$ \\
\hline Тe-129 & $3.6 \mathrm{E}-06$ & 2.1E-06 & Ba-140 & $2.1 \mathrm{E}-03$ & $1.3 \mathrm{E}-03$ \\
\hline $\mathrm{Te}-129 \mathrm{~m}$ & $4.9 \mathrm{E}-03$ & $3.1 \mathrm{E}-03$ & $\mathrm{Ba}-141$ & 2.6E-06 & $1.5 \mathrm{E}-06$ \\
\hline Te-131 & $2.9 \mathrm{E}-06$ & $1.7 \mathrm{E}-06$ & Ba-142 & $8.7 \mathrm{E}-07$ & $5.1 \mathrm{E}-07$ \\
\hline $\mathrm{Te}-131 \mathrm{~m}$ & 4.7E-04 & $2.9 \mathrm{E}-04$ & $\mathrm{La}-140$ & $8.0 \mathrm{E}-04$ & $5.0 \mathrm{E}-04$ \\
\hline Te-132 & 8.2E-04 & $5.1 \mathrm{E}-04$ & La-141 & $1.5 \mathrm{E}-06$ & $9.0 \mathrm{E}-07$ \\
\hline Te-133 & $1.3 \mathrm{E}-06$ & 7.4E-07 & La-142 & $5.6 \mathrm{E}-05$ & $3.4 \mathrm{E}-05$ \\
\hline $\mathrm{Te}-133 \mathrm{~m}$ & $2.8 \mathrm{E}-05$ & $1.6 \mathrm{E}-05$ & Ce-141 & $1.5 \mathrm{E}-03$ & $9.3 \mathrm{E}-04$ \\
\hline Te-134 & $8.0 \mathrm{E}-06$ & 4.7E-06 & Ce-143 & $2.4 \mathrm{E}-04$ & $1.5 \mathrm{E}-04$ \\
\hline I-122 & $1.3 \mathrm{E}-09$ & $5.2 \mathrm{E}-09$ & Ce-144 & $2.5 \mathrm{E}-02$ & $1.5 \mathrm{E}-02$ \\
\hline $\mathrm{I}-123$ & $8.1 \mathrm{E}-06$ & $3.6 \mathrm{E}-05$ & Pr-143 & $1.2 \mathrm{E}-03$ & 7.3E-04 \\
\hline I-125 & $1.5 \mathrm{E}-02$ & $6.4 \mathrm{E}-02$ & $\operatorname{Pr}-144$ & $4.3 \mathrm{E}-07$ & $2.5 \mathrm{E}-07$ \\
\hline
\end{tabular}


Table 4-7. 100-K Area--CAP88-PC Unit Dose Factors by Effective Release Height ${ }^{\mathrm{a}}$ (mrem/Ci).

\begin{tabular}{|c|c|c|c|c|c|}
\hline \multirow{2}{*}{ Nuclide } & \multicolumn{2}{|c|}{$\begin{array}{c}\text { Effective } \\
\text { Release Height }\end{array}$} & \multirow{2}{*}{ Nuclide } & \multicolumn{2}{|c|}{$\begin{array}{c}\text { Effective } \\
\text { Release Height }\end{array}$} \\
\hline & $\mathrm{H}_{\mathrm{eff}}<40 \mathrm{~m}$ & $\mathrm{H}_{\text {eff }} \geq 40 \mathrm{~m}$ & & $\mathrm{H}_{\mathrm{eff}}<40 \mathrm{~m}$ & $\mathrm{H}_{\text {eff }} \geq 40 \mathrm{~m}$ \\
\hline $\mathrm{Pr}-144 \mathrm{~m}$ & $2.2 \mathrm{E}-08$ & $1.3 \mathrm{E}-08$ & Po- $211^{c}$ & 0.0 & 0.0 \\
\hline Nd-147 & $1.2 \mathrm{E}-03$ & 7.2E-04 & Po- $212^{\mathrm{c}}$ & 0.0 & 0.0 \\
\hline Pm-147 & 2.1E-03 & $1.3 \mathrm{E}-03$ & Po- $213^{\mathrm{c}}$ & 0.0 & 0.0 \\
\hline Pm-148 & $1.7 \mathrm{E}-03$ & $1.1 \mathrm{E}-03$ & Po- $214^{\mathrm{c}}$ & 0.0 & 0.0 \\
\hline Pm-148m & $1.5 \mathrm{E}-02$ & $9.7 \mathrm{E}-03$ & Po- $215^{\mathrm{c}}$ & 0.0 & 0.0 \\
\hline Pm-149 & $2.2 \mathrm{E}-04$ & $1.3 \mathrm{E}-04$ & Po- $216^{\mathrm{c}}$ & 0.0 & 0.0 \\
\hline Pm-151 & $5.8 \mathrm{E}-05$ & $3.6 \mathrm{E}-05$ & Po-218 & $1.1 \mathrm{E}-09$ & $6.5 \mathrm{E}-10$ \\
\hline Sm-147 & $3.3 E+00$ & $2.0 \mathrm{E}+00$ & $A t-217^{\mathrm{c}}$ & 0.0 & 0.0 \\
\hline $\mathrm{Sm}-151$ & $1.5 \mathrm{E}-03$ & $9.0 \mathrm{E}-04$ & $R n-219^{d}$ & $(\mathrm{~Pb}-211)$ & $(\mathrm{Pb}-211)$ \\
\hline Sm-153 & $1.5 \mathrm{E}-04$ & $9.4 \mathrm{E}-05$ & $\operatorname{Rn}-220^{\mathrm{d}}$ & $(\mathrm{Pb}-212)$ & $(\mathrm{Pb}-212)$ \\
\hline Eu-152 & $4.8 \mathrm{E}-01$ & $3.0 \mathrm{E}-01$ & $R n-222$ & $1.4 \mathrm{E}-04$ & $7.1 \mathrm{E}-05$ \\
\hline $\mathrm{Eu}-152 \mathrm{~m}$ & $2.1 \mathrm{E}-05$ & $1.3 \mathrm{E}-05$ & Fr-221 & 7.1E-07 & 4.3E-07 \\
\hline Eu-154 & $3.9 \mathrm{E}-01$ & $2.5 \mathrm{E}-01$ & Fr-223 & $1.7 \mathrm{E}-05$ & $9.7 \mathrm{E}-06$ \\
\hline Eu-155 & $1.6 \mathrm{E}-02$ & $9.9 \mathrm{E}-03$ & $\mathrm{Ra}-223$ & $4.8 \mathrm{E}-01$ & $2.9 \mathrm{E}-01$ \\
\hline Eu-156 & $4.8 \mathrm{E}-03$ & $3.0 \mathrm{E}-03$ & $\mathrm{Ra}-224$ & $1.7 \mathrm{E}-01$ & $1.0 \mathrm{E}-01$ \\
\hline $\mathrm{Gd}-152^{\mathrm{b}}$ & (Pu-239) & (Pu-239) & $\mathrm{Ra}-225$ & $3.0 \mathrm{E}-01$ & $1.8 \mathrm{E}-01$ \\
\hline $\mathrm{Tb}-160$ & $1.3 \mathrm{E}-02$ & $8.0 \mathrm{E}-03$ & $\mathrm{Ra}-226$ & $9.1 \mathrm{E}-01$ & $5.6 \mathrm{E}-01$ \\
\hline Ho-166 & $1.8 \mathrm{E}-04$ & $1.1 \mathrm{E}-04$ & Ra-228 & $3.8 \mathrm{E}-01$ & $2.3 \mathrm{E}-01$ \\
\hline Ho- $166 \mathrm{~m}$ & $1.6 \mathrm{E}+00$ & $9.9 \mathrm{E}-01$ & Ac-225 & $2.8 \mathrm{E}-01$ & $1.7 \mathrm{E}-01$ \\
\hline Hf-181 & $4.8 \mathrm{E}-03$ & $3.0 \mathrm{E}-03$ & Ac- 227 & $2.9 \mathrm{E}+01$ & $1.8 \mathrm{E}+01$ \\
\hline W-181 & $8.9 \mathrm{E}-04$ & $5.6 \mathrm{E}-04$ & Ac-228 & $3.3 \mathrm{E}-03$ & $2.0 \mathrm{E}-03$ \\
\hline W-185 & $1.2 \mathrm{E}-03$ & 7.5E-04 & Th-227 & $5.2 \mathrm{E}-01$ & $3.1 \mathrm{E}-01$ \\
\hline W-187 & $1.0 \mathrm{E}-04$ & $6.3 \mathrm{E}-05$ & Th-228 & $1.1 \mathrm{E}+01$ & $6.7 \mathrm{E}+00$ \\
\hline Re-187 & $3.4 \mathrm{E}-05$ & 2.1E-05 & Th-229 & $3.1 \mathrm{E}+01$ & $1.9 \mathrm{E}+01$ \\
\hline Ir-192 & $1.1 \mathrm{E}-02$ & $6.8 \mathrm{E}-03$ & Th-230 & $1.1 \mathrm{E}+01$ & $6.7 \mathrm{E}+\infty 0$ \\
\hline $\mathrm{Hg}-203$ & $6.7 \mathrm{E}-03$ & $4.2 \mathrm{E}-03$ & Th-231 & $4.8 \mathrm{E}-05$ & $2.9 \mathrm{E}-05$ \\
\hline Tl-207 & $1.5 \mathrm{E}-09$ & $9.1 \mathrm{E}-10$ & Th-232 & $1.6 \mathrm{E}+01$ & $9.5 E+00$ \\
\hline T1-208 & $4.6 \mathrm{E}-08$ & $2.8 \mathrm{E}-08$ & Th-234 & 4.7E-03 & $2.9 \mathrm{E}-03$ \\
\hline Tl-209 & $2.9 \mathrm{E}-09$ & $1.8 \mathrm{E}-09$ & $\mathrm{~Pa}-231$ & $2.3 \mathrm{E}+01$ & $1.4 \mathrm{E}+01$ \\
\hline $\mathrm{Pb}-209$ & $3.3 \mathrm{E}-06$ & $2.0 \mathrm{E}-06$ & $\mathrm{~Pa}-233$ & $2.1 \mathrm{E}-03$ & $1.3 \mathrm{E}-03$ \\
\hline $\mathrm{Pb}-210$ & $2.8 \mathrm{E}+00$ & $1.8 \mathrm{E}+00$ & $\mathrm{~Pa}-234$ & $1.4 \mathrm{E}-04$ & $8.8 \mathrm{E}-05$ \\
\hline $\mathrm{Pb}-211$ & $1.1 \mathrm{E}-04$ & $6.5 \mathrm{E}-05$ & $\mathrm{~Pa}-234 \mathrm{~m}$ & $2.5 \mathrm{E}-14$ & $1.5 \mathrm{E}-14$ \\
\hline $\mathrm{Pb}-212$ & $6.8 \mathrm{E}-03$ & $4.1 \mathrm{E}-03$ & U-232 & $2.2 \mathrm{E}+01$ & $1.3 \mathrm{E}+01$ \\
\hline $\mathrm{Pb}-214$ & $9.1 \mathrm{E}-06$ & $5.2 \mathrm{E}-06$ & U-233 & $6.2 \mathrm{E}+00$ & $3.8 \mathrm{E}+00$ \\
\hline $\mathrm{Bi}-210$ & $8.9 \mathrm{E}-03$ & $5.4 \mathrm{E}-03$ & U-234 & $6.2 E+\infty 0$ & $3.7 \mathrm{E}+00$ \\
\hline Bi-211 & $2.0 \mathrm{E}-09$ & $1.2 \mathrm{E}-09$ & U-235 & $5.9 \mathrm{E}+\infty 0$ & $3.5 \mathrm{E}+00$ \\
\hline $\mathrm{Bi}-212$ & $6.4 \mathrm{E}-04$ & $3.8 \mathrm{E}-04$ & U-236 & $5.8 \mathrm{E}+\infty 0$ & $3.5 \mathrm{E}+\infty 0$ \\
\hline $\mathrm{Bi}-213$ & $2.0 \mathrm{E}-05$ & $1.2 \mathrm{E}-05$ & U-237 & $6.1 \mathrm{E}-04$ & $3.8 \mathrm{E}-04$ \\
\hline Bi-214 & $7.4 \mathrm{E}-06$ & $4.3 \mathrm{E}-06$ & $\mathrm{U}-238$ & $5.5 \mathrm{E}+00$ & $3.3 E+00$ \\
\hline Po-210 & $1.0 \mathrm{E}+00$ & $6.4 \mathrm{E}-01$ & $\mathrm{U}-240$ & $1.1 \mathrm{E}-04$ & $6.3 \mathrm{E}-05$ \\
\hline
\end{tabular}


Table 4-7. 100-K Area-CAP88-PC Unit Dose Factors by Effective Release Height ${ }^{\mathrm{a}}$ (mrem/Ci).

\begin{tabular}{|c|c|c|c|c|c|}
\hline \multirow{2}{*}{ Nuclide } & \multicolumn{2}{|c|}{$\begin{array}{c}\text { Effective } \\
\text { Release Height }\end{array}$} & \multirow{2}{*}{ Nuclide } & \multicolumn{2}{|c|}{$\begin{array}{c}\text { Effective } \\
\text { Release Height }\end{array}$} \\
\hline & $\mathrm{H}_{\text {eff }}<40 \mathrm{~m}$ & $\mathrm{H}_{\text {eff }}<40 \mathrm{~m}$ & & $\mathrm{H}_{\text {eff }}<40 \mathrm{~m}$ & $\mathrm{H}_{\text {eff }} \geq 40 \mathrm{~m}$ \\
\hline $\mathrm{Np}-237$ & $2.3 \mathrm{E}+01$ & $1.4 \mathrm{E}+01$ & & & \\
\hline$N p-238$ & $1.6 \mathrm{E}-03$ & $9.6 \mathrm{E}-04$ & & & \\
\hline Np-239 & 2.4E-04 & $1.5 \mathrm{E}-04$ & & & \\
\hline $\mathrm{Np}-240$ & $1.9 \mathrm{E}-05$ & $1.1 \mathrm{E}-05$ & & & \\
\hline $\mathrm{Np}-240 \mathrm{~m}$ & 1.4E-07 & $8.1 \mathrm{E}-08$ & & & \\
\hline Pu-236 & $4.0 \mathrm{E}+\infty 0$ & $2.4 \mathrm{E}+00$ & & & \\
\hline Pu-238 & $1.5 \mathrm{E}+01$ & $9.2 \mathrm{E}+00$ & & & \\
\hline Pu-239 & $1.6 \mathrm{E}+01$ & $9.9 \mathrm{E}+00$ & & & - \\
\hline Pu-240 & $1.6 \mathrm{E}+01$ & $9.9 \mathrm{E}+00$ & & & \\
\hline Pu-241 & $2.6 \mathrm{E}-01$ & $1.6 \mathrm{E}-01$ & & & \\
\hline Pu-242 & $1.6 \mathrm{E}+01$ & $9.4 \mathrm{E}+00$ & & & \\
\hline $\mathrm{Pu}-243$ & 9.4E-06 & $5.6 \mathrm{E}-06$ & & & \\
\hline Pu-244 & $1.6 \mathrm{E}+01$ & $9.3 E+00$ & & & \\
\hline Am-241 & $2.5 \mathrm{E}+01$ & $1.5 \mathrm{E}+01$ & & & \\
\hline Am-242 & $2.5 \mathrm{E}-03$ & $1.5 \mathrm{E}-03$ & & & \\
\hline $\mathrm{Am}-242 \mathrm{~m}$ & $2.4 \mathrm{E}+01$ & $1.5 \mathrm{E}+01$ & & & \\
\hline Am-243 & $2.5 \mathrm{E}+01$ & $1.5 \mathrm{E}+01$ & & & \\
\hline $\mathrm{Cm}-242$ & $8.2 \mathrm{E}-01$ & $4.9 \mathrm{E}-01$ & & & \\
\hline $\mathrm{Cm}-243$ & $1.7 \mathrm{E}+01$ & $1.0 \mathrm{E}+01$ & & & \\
\hline $\mathrm{Cm}-244$ & $1.3 \mathrm{E}+01$ & $8.0 \mathrm{E}+00$ & & & \\
\hline $\mathrm{Cm}-245$ & $2.6 \mathrm{E}+01$ & $1.6 \mathrm{E}+01$ & & & \\
\hline $\mathrm{Cm}-246$ & $2.6 \mathrm{E}+01$ & $1.6 \mathrm{E}+01$ & & & \\
\hline $\mathrm{Cm}-247$ & $2.4 \mathrm{E}+01$ & $1.4 \mathrm{E}+01$ & & & \\
\hline $\mathrm{Cm}-248$ & $9.5 \mathrm{E}+01$ & $5.7 \mathrm{E}+01$ & & & \\
\hline Cf-252 & $7.1 \mathrm{E}+\infty 0$ & $4.3 \mathrm{E}+\infty 0$ & & & \\
\hline
\end{tabular}

2 The effective release height, $x$, is in meters.

- CAP88-PC Ver. 1.0 does not calculate doses for Be-10, Se-79, Cd-113, Cd-113m, and Gd-152. Recommend substituting the unit dose factors of the nuclides in parentheses.

c Extremely short lived nuclide, resulting in an offsite dose of zero.

d Short lived radon nuclides only provide the mechanism to circumvent the emission control device. The unit dose factors for the recommended progeny should be used instead. However, $1 \mathrm{ci}$ of $\mathrm{Rn}$ does not equal $1 \mathrm{ci}$ of $\mathrm{Pb}$. Multiply the activity of $\mathrm{Rn}-219$ by 0.0018 to get the activity of $\mathrm{Pb}-211$. Multiply the activity of $\mathrm{Rn}-220$ by 0.0014 to get the activity of $\mathrm{Pb}-212$. 
Table 4-8. 100-N Area--CAP88-PC Unit Dose Factors by Effective Release Height ${ }^{\mathrm{a}}$ (mrem/Ci).

\begin{tabular}{|c|c|c|c|c|c|}
\hline \multirow{2}{*}{ Nuclide } & \multicolumn{2}{|c|}{$\begin{array}{c}\text { Effective } \\
\text { Release Height }\end{array}$} & \multirow{2}{*}{ Nuclide } & \multicolumn{2}{|c|}{$\begin{array}{c}\text { Effective } \\
\text { Release Height }\end{array}$} \\
\hline & $\mathrm{H}_{\mathrm{eff}}<40 \mathrm{~m}$ & $\mathrm{H}_{\text {eff } \geq 40 \mathrm{~m}}$ & & $\mathrm{H}_{\mathrm{eff}}<40 \mathrm{~m}$ & $\mathrm{H}_{\mathrm{eff}} \geq 40 \mathrm{~m}$ \\
\hline H-3 & $4.0 \mathrm{E}-05$ & $2.3 \mathrm{E}-05$ & $\mathrm{Kr}-85$ & $8.9 \mathrm{E}-08$ & $5.2 \mathrm{E}-08$ \\
\hline $\mathrm{Be}-7$ & $3.0 \mathrm{E}-04$ & $2.2 \mathrm{E}-04$ & $\mathrm{Kr}-85 \mathrm{~m}$ & $3.2 \mathrm{E}-06$ & $1.9 \mathrm{E}-06$ \\
\hline $\mathrm{Be}-10^{\mathrm{b}}$ & $(\mathrm{Sr}-90)$ & $(\mathrm{Sr}-90)$ & $\mathrm{Kr}-87$ & $9.4 \mathrm{E}-06$ & $5.6 \mathrm{E}-06$ \\
\hline C-11 & $1.7 \mathrm{E}-06$ & $1.3 \mathrm{E}-06$ & $\mathrm{Kr}-88$ & $3.9 \mathrm{E}-05$ & $2.3 \mathrm{E}-05$ \\
\hline C-14 & $3.1 \mathrm{E}-03$ & $1.8 \mathrm{E}-03$ & $\mathrm{Kr}-89$ & $3.3 \mathrm{E}-08$ & $2.1 \mathrm{E}-08$ \\
\hline$C-15^{c}$ & 0.0 & 0.0 & $\mathrm{Kr}-90$ & $2.5 \mathrm{E}-19$ & $1.5 \mathrm{E}-19$ \\
\hline$N-13$ & $4.9 \mathrm{E}-07$ & $3.6 \mathrm{E}-07$ & $\mathrm{Rb}-86$ & $4.4 \mathrm{E}-03$ & $3.3 \mathrm{E}-03$ \\
\hline $0-15$ & 7.4E-10 & $5.4 \mathrm{E}-10$ & $\mathrm{Rb}-87$ & $1.5 \mathrm{E}-02$ & $1.1 \mathrm{E}-02$ \\
\hline F-18 & $1.6 \mathrm{E}-05$ & $1.1 \mathrm{E}-05$ & $\mathrm{Rb}-88$ & $1.1 \mathrm{E}-06$ & $8.4 \mathrm{E}-07$ \\
\hline $\mathrm{Na}-22$ & $2.0 \mathrm{E}-01$ & $1.5 \mathrm{E}-01$ & $\mathrm{Rb}-89$ & $2.4 \mathrm{E}-06$ & $1.7 \mathrm{E}-06$ \\
\hline $\mathrm{Na}-24$ & $3.3 \mathrm{E}-04$ & $2.4 \mathrm{E}-04$ & Rb-90 & $1.1 \mathrm{E}-08$ & $8.0 \mathrm{E}-09$ \\
\hline P-32 & $4.5 \mathrm{E}-03$ & $3.3 \mathrm{E}-03$ & $\mathrm{Rb}-90 \mathrm{~m}$ & $1.8 \mathrm{E}-07$ & $1.4 \mathrm{E}-07$ \\
\hline S-35 & $6.3 \mathrm{E}-04$ & 4.7E-04 & Sr-89 & $2.7 \mathrm{E}-03$ & $2.0 \mathrm{E}-03$ \\
\hline Ar- 41 & $1.8 \mathrm{E}-05$ & $1.1 \mathrm{E}-05$ & Sr-90 & $1.7 \mathrm{E}-01$ & $1.3 \mathrm{E}-01$ \\
\hline $\mathrm{K}-40$ & $1.9 \mathrm{E}-01$ & $1.4 \mathrm{E}-01$ & Sr-91 & $6.8 \mathrm{E}-05$ & $4.9 \mathrm{E}-05$ \\
\hline $\mathrm{Ca}-41$ & $1.6 \mathrm{E}-05$ & $1.2 \mathrm{E}-05$ & Sr -92 & $4.0 \mathrm{E}-05$ & $2.9 \mathrm{E}-05$ \\
\hline Sc-46 & $1.9 \mathrm{E}-02$ & $1.4 \mathrm{E}-02$ & Y-90 & $5.3 \mathrm{E}-04$ & $3.8 \mathrm{E}-04$ \\
\hline Cr-51 & $1.4 \mathrm{E}-04$ & $1.0 \mathrm{E}-04$ & Y-90m & $1.4 \mathrm{E}-05$ & $1.0 \mathrm{E}-05$ \\
\hline Mn-54 & $2.5 \mathrm{E}-02$ & $1.9 \mathrm{E}-02$ & Y-91 & $3.9 \mathrm{E}-03$ & $2.8 \mathrm{E}-03$ \\
\hline $\mathrm{Mn}-56$ & 4.4E-05. & $3.2 \mathrm{E}-05$ & $\mathrm{Y}-91 \mathrm{~m}$ & $3.6 \mathrm{E}-06$ & $2.6 \mathrm{E}-06$ \\
\hline $\mathrm{Fe}-55$ & $3.6 \mathrm{E}-04$ & $2.6 \mathrm{E}-04$ & Y-92 & $3.5 \mathrm{E}-05$ & $2.5 \mathrm{E}-05$ \\
\hline $\mathrm{Fe}-59$ & $7.3 \mathrm{E}-03$ & $5.4 \mathrm{E}-03$ & Y-93 & $9.0 \mathrm{E}-05$ & $6.4 \mathrm{E}-05$ \\
\hline $\mathrm{Co}-57$ & $4.4 \mathrm{E}-03$ & $3.2 \mathrm{E}-03$ & Zr-93 & $2.0 \mathrm{E}-03$ & $1.4 \mathrm{E}-03$ \\
\hline Co-58 & $8.4 \mathrm{E}-03$ & $6.2 \mathrm{E}-03$ & Zr-95 & $6.0 \mathrm{E}-03$ & $4.4 \mathrm{E}-03$ \\
\hline Co- 60 & $3.8 \mathrm{E}-01$ & $2.8 \mathrm{E}-01$ & $\mathrm{Nb}-93 \mathrm{~m}$ & $3.2 \mathrm{E}-03$ & 2.3E-03 \\
\hline $\mathrm{Ni}-59$ & $4.8 \mathrm{E}-04$ & $3.5 \mathrm{E}-04$ & $\mathrm{Nb}-94$ & $1.3 \mathrm{E}+00$ & $9.2 \mathrm{E}-01$ \\
\hline $\mathrm{Ni}-63$ & $4.0 \mathrm{E}-04$ & $3.0 \mathrm{E}-04$ & Nb-95 & $7.4 \mathrm{E}-03$ & $5.5 \mathrm{E}-03$ \\
\hline $\mathrm{Ni}-65$ & $1.7 \mathrm{E}-05$ & $1.3 \mathrm{E}-05$ & $\mathrm{Nb}-95 \mathrm{~m}$ & $4.6 \mathrm{E}-04$ & $3.4 \mathrm{E}-04$ \\
\hline $\mathrm{Cu}-64$ & $2.3 \mathrm{E}-05$ & $1.7 \mathrm{E}-05$ & Nb-97 & $8.1 \mathrm{E}-06$ & $5.9 \mathrm{E}-06$ \\
\hline $\mathrm{Zn}-65$ & $3.8 \mathrm{E}-02$ & $2.8 \mathrm{E}-02$ & $\mathrm{Nb}-97 \mathrm{~m}$ & $1.3 \mathrm{E}-13$ & $9.6 \mathrm{E}-14$ \\
\hline $\mathrm{Zn}-69 \mathrm{~m}$ & $6.3 \mathrm{E}-05$ & $4.5 \mathrm{E}-05$ & Mo-93 & $4.3 \mathrm{E}-03$ & $3.2 \mathrm{E}-03$ \\
\hline $\mathrm{Zn}-69$ & $6.8 \mathrm{E}-07$ & $4.9 \mathrm{E}-07$ & Mo-99 & 2.7E-04 & $1.9 \mathrm{E}-04$ \\
\hline Ga-67 & $9.5 \mathrm{E}-05$ & $6.9 \mathrm{E}-05$ & Tc-97 & $8.9 \mathrm{E}-03$ & $6.6 \mathrm{E}-03$ \\
\hline As-76 & $2.1 \mathrm{E}-04$ & $1.5 \mathrm{E}-04$ & Tc-99 & $3.5 \mathrm{E}-02$ & $2.6 \mathrm{E}-02$ \\
\hline $\mathrm{Se}-79^{\mathrm{b}}$ & (Pu-241) & (Pu-241) & Tc-99m & $5.9 \mathrm{E}-06$ & $4.3 \mathrm{E}-06$ \\
\hline $\mathrm{Br}-82$ & $5.0 \mathrm{E}-04$ & $3.6 \mathrm{E}-04$ & Tc-101 & $3.0 \mathrm{E}-07$ & $2.2 \mathrm{E}-07$ \\
\hline $\mathrm{Br}-83$ & $1.3 \mathrm{E}-07$ & $9.5 \mathrm{E}-08$ & Ru-97 & $1.0 \mathrm{E}-04$ & 7.5E-05 \\
\hline $\mathrm{Br}-84$ & $6.4 \mathrm{E}-06$ & 4.7E-06 & Ru-103 & $2.9 \mathrm{E}-03$ & $2.1 \mathrm{E}-03$ \\
\hline $\mathrm{Br}-85$ & $5.1 \mathrm{E}-10$ & $3.8 \mathrm{E}-10$ & $\mathrm{Ru}-105$ & $3.9 \mathrm{E}-05$ & $2.8 \mathrm{E}-05$ \\
\hline $\mathrm{Kr}-83 \mathrm{~m}$ & $3.3 \mathrm{E}-09$ & $1.9 \mathrm{E}-09$ & Ru-106 & $2.4 \mathrm{E}-02$ & $1.7 \mathrm{E}-02$ \\
\hline
\end{tabular}


HNF-3602, Vol. 1

Table 4-8. 100-N Area--CAP88-PC Unit Dose Factors by Effective Release Height ${ }^{\mathrm{a}}$ $(\mathrm{mrem} / \mathrm{Ci})$.

\begin{tabular}{|c|c|c|c|c|c|}
\hline \multirow{2}{*}{ Nuclide } & \multicolumn{2}{|c|}{$\begin{array}{c}\text { Effective } \\
\text { Release Height }\end{array}$} & \multirow{2}{*}{ Nuclide } & \multicolumn{2}{|c|}{$\begin{array}{c}\text { Effective } \\
\text { Release Height }\end{array}$} \\
\hline & $\mathrm{H}_{\mathrm{eff}}<40 \mathrm{~m}$ & $\mathrm{H}_{\text {eff }} \geq 40 \mathrm{~m}$ & & $\mathrm{H}_{\mathrm{eff}}<40 \mathrm{~m}$ & $\mathrm{H}_{\text {eff }} \geq 40 \mathrm{~m}$ \\
\hline Rh-103m & $8.7 \mathrm{E}-08$ & $6.3 \mathrm{E}-08$ & I-129 & $1.7 \mathrm{E}-01$ & $7.0 \mathrm{E}-01$ \\
\hline Rh-105 & $7.5 \mathrm{E}-05$ & $5.4 \mathrm{E}-05$ & I-130 & $6.2 \mathrm{E}-05$ & $2.5 \mathrm{E}-04$ \\
\hline $\mathrm{Rh}-105 \mathrm{~m}$ & $2.1 \mathrm{E}-17$ & $1.6 \mathrm{E}-17$ & I-131 & $5.6 \mathrm{E}-03$ & $2.3 \mathrm{E}-02$ \\
\hline Rh-106 & $2.7 \mathrm{E}-21$ & $2.0 \mathrm{E}-21$ & I-132 & $8.4 \mathrm{E}-06$ & 3.3E-05 \\
\hline Pd-107 & $5.8 \mathrm{E}-04$ & $4.1 \mathrm{E}-04$ & I-133 & $6.4 \mathrm{E}-05$ & $2.6 \mathrm{E}-04$ \\
\hline Pd-109 & $5.4 \mathrm{E}-05$ & $3.9 \mathrm{E}-05$ & I-134 & $2.1 \mathrm{E}-06$ & $8.1 \mathrm{E}-06$ \\
\hline $\mathrm{Ag}-109 \mathrm{~m}$ & $1.8 \mathrm{E}-19$ & $1.3 \mathrm{E}-19$ & I-135 & $2.0 \mathrm{E}-05$ & $8.1 \mathrm{E}-05$ \\
\hline Ag-110 & $3.3 \mathrm{E}-25$ & $2.5 \mathrm{E}-25$ & $\mathrm{Xe}-122$ & $1.7 \mathrm{E}-06$ & $9.9 \mathrm{E}-07$ \\
\hline $\mathrm{Ag}-110 \mathrm{~m}$ & $7.4 \mathrm{E}-02$ & $5.5 \mathrm{E}-02$ & $\mathrm{Xe}-123$ & $9.5 \mathrm{E}-06$ & $5.6 \mathrm{E}-06$ \\
\hline Ag-111 & $1.6 \mathrm{E}-03$ & $1.2 \mathrm{E}-03$ & $\mathrm{Xe}-125$ & $6.1 \mathrm{E}-06$ & $3.6 \mathrm{E}-06$ \\
\hline $\mathrm{Cd}-113^{\mathrm{b}}$ & (Pu-241) & (Pu-241) & $\mathrm{Xe}-127$ & $6.9 \mathrm{E}-06$ & $4.1 \mathrm{E}-06$ \\
\hline $\mathrm{Cd}-113 \mathrm{~m}^{\mathrm{b}}$ & (Pu-241) & (Pu-241) & $\mathrm{Xe}-131 \mathrm{~m}$ & $2.5 \mathrm{E}-07$ & $1.5 \mathrm{E}-07$ \\
\hline Cd-115 & $3.0 \mathrm{E}-04$ & $2.1 \mathrm{E}-04$ & $X e-133$ & $9.3 \mathrm{E}-07$ & $5.4 \mathrm{E}-07$ \\
\hline Cd-115m & $5.5 \mathrm{E}-03$ & $4.0 \mathrm{E}-03$ & $\mathrm{Xe}-133 \mathrm{~m}$ & $7.9 \mathrm{E}-07$ & $4.6 \mathrm{E}-07$ \\
\hline In-113m & $4.2 \mathrm{E}-06$ & $3.0 \mathrm{E}-06$ & $\mathrm{Xe}-135$ & 5.7E-06 & $3.4 \mathrm{E}-06$ \\
\hline In-115 & 9.3E-02 & $6.8 \mathrm{E}-02$ & $\mathrm{Xe}-135 \mathrm{~m}$ & $5.3 \mathrm{E}-07$ & $3.3 \mathrm{E}-07$ \\
\hline $\ln -115 \mathrm{~m}$ & $9.1 \mathrm{E}-06$ & $6.5 \mathrm{E}-06$ & $\mathrm{Xe}-137$ & $7.9 \mathrm{E}-09$ & $4.9 \mathrm{E}-09$ \\
\hline Sn-113 & $3.4 \mathrm{E}-03$ & $2.5 \mathrm{E}-03$ & Xe-138 & $1.4 \mathrm{E}-06$ & $8.5 \mathrm{E}-07$ \\
\hline Sn-123 & $8.0 \mathrm{E}-05$ & $5.9 \mathrm{E}-05$ & Cs-134 & $1.6 \mathrm{E}-01$ & $1.1 \mathrm{E}-01$ \\
\hline Sn-125 & $3.7 \mathrm{E}-03$ & $2.7 \mathrm{E}-03$ & $\mathrm{Cs}-134 \mathrm{~m}$ & $1.5 \mathrm{E}-06$ & $1.1 \mathrm{E}-06$ \\
\hline Sn-126 & $7.3 \mathrm{E}-02$ & $5.4 \mathrm{E}-02$ & Cs-135 & $6.8 \mathrm{E}-03$ & $5.0 \mathrm{E}-03$ \\
\hline Sb-124 & $1.3 \mathrm{E}-02$ & $9.8 \mathrm{E}-03$ & Cs-136 & $5.7 \mathrm{E}-03$ & $4.2 \mathrm{E}-03$ \\
\hline $\mathrm{Sb}-125$ & $4.1 \mathrm{E}-02$ & $3.0 \mathrm{E}-02$ & Cs-137 & 4.1E-02 & $3.0 \mathrm{E}-02$ \\
\hline$S b-126$ & $5.1 \mathrm{E}-03$ & $3.7 \mathrm{E}-03$ & Cs-138 & $8.8 \mathrm{E}-06$ & $6.4 \mathrm{E}-06$ \\
\hline $\mathrm{Sb}-126 \mathrm{~b}$ & $2.4 \mathrm{E}-06$ & $1.8 \mathrm{E}-06$ & Cs-139 & $1.4 \mathrm{E}-07$ & $1.0 \mathrm{E}-07$ \\
\hline Sb-127 & $7.2 \mathrm{E}-04$ & $5.3 E-04$ & $\mathrm{Ba}-133$ & $1.2 \mathrm{E}-01$ & $8.5 \mathrm{E}-02$ \\
\hline $\mathrm{Te}-125 \mathrm{~m}$ & $1.6 \mathrm{E}-03$ & $1.2 \mathrm{E}-03$ & Ba-133m & $4.9 \mathrm{E}-05$ & $3.5 \mathrm{E}-05$ \\
\hline $\mathrm{Te}-127$ & $1.3 \mathrm{E}-05$ & $9.2 \mathrm{E}-06$ & $\mathrm{Ba}-137 \mathrm{~m}$ & $2.2 \mathrm{E}-09$ & $1.6 \mathrm{E}-09$ \\
\hline $\mathrm{Te}-127 \mathrm{~m}$ & $3.9 \mathrm{E}-03$ & $2.9 \mathrm{E}-03$ & Ba-139 & $3.2 \mathrm{E}-06$ & 2.3E-06 \\
\hline $\mathrm{Te}-129$ & $2.2 \mathrm{E}-06$ & $1.6 \mathrm{E}-06$ & Ba-140 & $1.6 \mathrm{E}-03$ & $1.2 \mathrm{E}-03$ \\
\hline $\mathrm{Te}-129 \mathrm{~m}$ & $3.9 \mathrm{E}-03$ & $2.8 \mathrm{E}-03$ & $\mathrm{Ba}-141$ & $1.2 \mathrm{E}-06$ & $8.9 \mathrm{E}-07$ \\
\hline $\mathrm{Te}-131$ & $1.4 \mathrm{E}-06$ & $1.0 \mathrm{E}-06$ & Ba-142 & $4.9 \mathrm{E}-07$ & $3.6 \mathrm{E}-07$ \\
\hline $\mathrm{Te}-131 \mathrm{~m}$ & $3.7 \mathrm{E}-04$ & $2.6 \mathrm{E}-04$ & La-140 & $6.2 \mathrm{E}-04$ & $4.5 \mathrm{E}-04$ \\
\hline $\mathrm{Te}-132$ & $6.4 \mathrm{E}-04$ & $4.6 \mathrm{E}-04$ & La-141 & $1.1 \mathrm{E}-06$ & $7.7 \mathrm{E}-07$ \\
\hline $\mathrm{Te}-133$ & $6.6 \mathrm{E}-07$ & $4.9 \mathrm{E}-07$ & La-142 & $3.6 \mathrm{E}-05$ & $2.6 \mathrm{E}-05$ \\
\hline $\mathrm{Te}-133 \mathrm{~m}$ & $1.6 \mathrm{E}-05$ & $1.1 \mathrm{E}-05$ & Ce-141 & $1.2 \mathrm{E}-03$ & $8.5 \mathrm{E}-04$ \\
\hline $\mathrm{Te}-134$ & 4.2E-06 & $3.1 \mathrm{E}-06$ & Ce-143 & $1.8 \mathrm{E}-04$ & $1.3 \mathrm{E}-04$ \\
\hline I-122 & $1.0 \mathrm{E}-09$ & $3.9 \mathrm{E}-09$ & Ce-144 & $1.9 \mathrm{E}-02$ & $1.4 \mathrm{E}-02$ \\
\hline I-123 & $5.7 \mathrm{E}-06$ & 2.3E-05 & Pr-143 & $9.1 \mathrm{E}-04$ & $6.7 \mathrm{E}-04$ \\
\hline I- 125 & $1.0 \mathrm{E}-02$ & $4.2 \mathrm{E}-02$ & $\mathrm{Pr}-144$ & $2.0 \mathrm{E}-07$ & $1.5 \mathrm{E}-07$ \\
\hline
\end{tabular}


Table 4-8. 100-N Area--CAP88-PC Unit Dose Factors by Effective Release Height ${ }^{\mathrm{a}}$ (mrem/Ci).

\begin{tabular}{|c|c|c|c|c|c|}
\hline \multirow{2}{*}{ Nuclide } & \multicolumn{2}{|c|}{$\begin{array}{c}\text { Effective } \\
\text { Release Height }\end{array}$} & \multirow{2}{*}{ Nuclide } & \multicolumn{2}{|c|}{$\begin{array}{c}\text { Effective } \\
\text { Release Height }\end{array}$} \\
\hline & $\mathrm{H}_{\mathrm{eff}}<40 \mathrm{~m}$ & $\mathrm{H}_{\text {eff }} \geq 40 \mathrm{~m}$ & & $\mathrm{H}_{\mathrm{eff}}<40 \mathrm{~m}$ & $\mathrm{H}_{\text {eff }} \geq 40 \mathrm{~m}$ \\
\hline Pr-144m & $1.5 \mathrm{E}-08$ & $1.1 \mathrm{E}-08$ & Po-211 ${ }^{\mathrm{c}}$ & 0.0 & 0.0 \\
\hline $\mathrm{Nd}-147$ & $9.0 \mathrm{E}-04$ & $6.5 \mathrm{E}-04$ & Po-212 ${ }^{\mathrm{c}}$ & 0.0 & 0.0 \\
\hline Pm-147 & 1.7E-03 & $1.2 \mathrm{E}-03$ & Po-213 ${ }^{\mathrm{c}}$ & 0.0 & 0.0 \\
\hline Pm-148 & $1.3 \mathrm{E}-03$ & $9.7 \mathrm{E}-04$ & Po-214 & 0.0 & 0.0 \\
\hline $\mathrm{Pm}-148 \mathrm{~m}$ & $1.2 \mathrm{E}-02$ & $8.8 \mathrm{E}-03$ & Po-215 & 0.0 & 0.0 \\
\hline $\mathrm{Pm}-149$ & $1.7 \mathrm{E}-04$ & $1.2 \mathrm{E}-04$ & Po-216 ${ }^{\mathrm{C}}$ & 0.0 & 0.0 \\
\hline Pm-151 & $4.5 \mathrm{E}-05$ & $3.3 \mathrm{E}-05$ & Po-218 & $9.4 \mathrm{E}-10$ & 7.0E-10 \\
\hline $\mathrm{Sm}-147$ & $2.6 \mathrm{E}+00$ & $1.8 \mathrm{E}+00$ & At $-217^{c}$ & 0.0 & 0.0 \\
\hline Sm-151 & $1.2 \mathrm{E}-03$ & $8.2 \mathrm{E}-04$ & $\mathrm{Rn}-219^{\mathrm{d}}$ & $(\mathrm{Pb}-211)$ & $(\mathrm{Pb}-211)$ \\
\hline $\mathrm{Sm}-153$ & $1.2 \mathrm{E}-04$ & $8.5 \mathrm{E}-05$ & $R n-220^{d}$ & $(\mathrm{~Pb}-212)$ & $(\mathrm{Pb}-212)$ \\
\hline Eu-152 & $3.7 \mathrm{E}-01$ & $2.8 \mathrm{E}-01$ & $\mathrm{Rn}-222$ & $1.1 \mathrm{E}-04$ & $6.6 \mathrm{E}-05$ \\
\hline Eu-152m & $1.6 \mathrm{E}-05$ & $1.1 \mathrm{E}-05$ & Fr-221 & $5.6 \mathrm{E}-07$ & 4.1E-07 \\
\hline Eu-154 & $3.0 \mathrm{E}-01$ & $2.2 \mathrm{E}-01$ & Fr-223 & $7.8 \mathrm{E}-06$ & $5.7 \mathrm{E}-06$ \\
\hline Eu-155 & $1.2 \mathrm{E}-02$ & $9.0 \mathrm{E}-03$ & Ra-223 & $3.7 \mathrm{E}-01$ & $2.6 \mathrm{E}-01$ \\
\hline Eu-156 & $3.7 \mathrm{E}-03$ & $2.8 \mathrm{E}-03$ & $\mathrm{Ra}-224$ & $1.3 \mathrm{E}-01$ & $9.4 \mathrm{E}-02$ \\
\hline $\mathrm{Gd}-152^{\mathrm{b}}$ & $(\mathrm{Pu}-239)$ & (Pu-239) & Ra-225 & 2.3E-01 & $1.7 \mathrm{E}-01$ \\
\hline $\mathrm{Tb}-160$ & $9.9 \mathrm{E}-03$ & 7.3E-03 & $\mathrm{Ra}-226$ & 7.1E-01 & $5.1 \mathrm{E}-01$ \\
\hline Ho-166 & $1.4 \mathrm{E}-04$ & $9.8 \mathrm{E}-05$ & Ra-228 & $2.9 \mathrm{E}-01$ & $2.1 \mathrm{E}-01$ \\
\hline Ho-166m & $1.2 \mathrm{E}+00$ & $9.1 \mathrm{E}-01$. & Ac- 225 & $2.2 \mathrm{E}-01$ & $1.5 \mathrm{E}-01$ \\
\hline Hf-181 & $3.8 \mathrm{E}-03$ & $2.8 \mathrm{E}-03$ & Ac-227 & $2.3 E+01$ & $1.6 \mathrm{E}+01$ \\
\hline W-181 & $7.0 \mathrm{E}-04$ & $5.2 \mathrm{E}-04$ & Ac-228 & $2.4 \mathrm{E}-03$ & $1.7 \mathrm{E}-03$ \\
\hline W-185 & $9.3 \mathrm{E}-04$ & $6.8 \mathrm{E}-04$ & Th-227 & $4.0 \mathrm{E}-01$ & $2.8 \mathrm{E}-01$ \\
\hline W-187 & $7.9 \mathrm{E}-05$ & 5.7E-05 & Th-228 & $8.6 \mathrm{E}+00$ & $6.1 E+00$ \\
\hline Re-187 & $2.7 \mathrm{E}-05$ & $2.0 \mathrm{E}-05$ & Th-229 & $2.4 \mathrm{E}+01$ & $1.7 \mathrm{E}+01$ \\
\hline Ir-192 & $8.4 \mathrm{E}-03$ & $6.2 \mathrm{E}-03$ & Th-230 & $8.6 \mathrm{E}+00$ & $6.1 E+00$ \\
\hline $\mathrm{Hg}-203$ & $5.2 \mathrm{E}-03$ & $3.9 \mathrm{E}-03$ & Th-231 & $3.7 \mathrm{E}-05$ & $2.6 \mathrm{E}-05$ \\
\hline Tl-207 & $1.2 \mathrm{E}-09$ & $8.8 \mathrm{E}-10$ & Th-232 & $1.2 \mathrm{E}+01$ & $8.7 \mathrm{E}+00$ \\
\hline $\mathrm{Tl}-208$ & $4.1 \mathrm{E}-08$ & $3.0 \mathrm{E}-08$ & Th-234 & 3.7E-03 & $2.7 \mathrm{E}-03$ \\
\hline Tl-209 & $2.9 \mathrm{E}-09$ & 2.1E-09 & $\mathrm{Pa}-231$ & $1.8 \mathrm{E}+01$ & $1.3 \mathrm{E}+01$ \\
\hline $\mathrm{Pb}-209$ & $2.4 \mathrm{E}-06$ & $1.7 \mathrm{E}-06$ & $\mathrm{~Pa}-233$ & $1.6 \mathrm{E}-03$ & $1.2 \mathrm{E}-03$ \\
\hline $\mathrm{Pb}-210$ & $2.2 \mathrm{E}+00$ & $1.6 \mathrm{E}+00$ & $\mathrm{~Pa}-234$ & $1.1 \mathrm{E}-04$ & $7.7 \mathrm{E}-05$ \\
\hline $\mathrm{Pb}-211$ & 5.7E-05 & 4.1E-05 & $\mathrm{Pa}-234 \mathrm{~m}$ & 3.4E-14 & $2.5 \mathrm{E}-14$ \\
\hline $\mathrm{Pb}-212$ & $5.1 \mathrm{E}-03$ & $3.6 \mathrm{E}-03$ & U-232 & $1.7 \mathrm{E}+01$ & $1.2 \mathrm{E}+01$ \\
\hline $\mathrm{Pb}-214$ & 4.3E-06 & $3.1 \mathrm{E}-06$ & U-233 & $4.8 \mathrm{E}+00$ & $3.4 \mathrm{E}+00$ \\
\hline $\mathrm{Bi}-210$ & $6.9 \mathrm{E}-03$ & $4.9 \mathrm{E}-03$ & U-234 & $4.8 \mathrm{E}+\infty 0$ & $3.4 \mathrm{E}+00$ \\
\hline $\mathrm{Bi}-211$ & $2.0 \mathrm{E}-09$ & $1.4 \mathrm{E}-09$ & U-235 & $4.6 \mathrm{E}+\infty 0$ & $3.2 \mathrm{E}+\infty 0$ \\
\hline $\mathrm{Bi}-212$ & 3.7E-04 & 2.7E-04 & U-236 & $4.5 \mathrm{E}+00$ & $3.2 \mathrm{E}+\infty 0$ \\
\hline $\mathrm{Bi}-213$ & $1.1 \mathrm{E}-05$ & $7.8 \mathrm{E}-06$ & U-237 & $4.8 \mathrm{E}-04$ & $3.5 \mathrm{E}-04$ \\
\hline $\mathrm{Bi}-214$ & $3.4 \mathrm{E}-06$ & $2.5 \mathrm{E}-06$ & $\mathrm{U}-238$ & $4.2 \mathrm{E}+00$ & $3.0 \mathrm{E}+\infty 0$ \\
\hline $\mathrm{P}_{0}-210$ & $8.1 \mathrm{E}-01$ & $5.9 \mathrm{E}-01$ & $\mathrm{U}-240$ & $7.9 \mathrm{E}-05$ & $5.6 \mathrm{E}-05$ \\
\hline
\end{tabular}


HNF-3602, Vol. 1

Table 4-8. 100-N Area--CAP88-PC Unit Dose Factors by Effective Release Height ${ }^{\mathrm{a}}$ (mrem/Ci).

\begin{tabular}{|c|c|c|c|c|c|}
\hline \multirow{2}{*}{ Nuclide } & \multicolumn{2}{|c|}{$\begin{array}{c}\text { Effective } \\
\text { Release Height }\end{array}$} & \multirow{2}{*}{ Nuclide } & \multicolumn{2}{|c|}{$\begin{array}{c}\text { Effective } \\
\text { Release Height }\end{array}$} \\
\hline & $\mathrm{H}_{\mathrm{eff}}<40 \mathrm{~m}$ & $\mathrm{H}_{\text {eff }} \geq 40 \mathrm{~m}$ & & $\mathrm{H}_{\mathrm{eff}}<40 \mathrm{~m}$ & $\mathrm{H}_{\text {eff }} \geq 40 \mathrm{~m}$ \\
\hline Np-237 & $1.8 \mathrm{E}+01$ & $1.3 \mathrm{E}+01$ & & & \\
\hline $\mathrm{Np}-238$ & $1.2 \mathrm{E}-03$ & $8.7 \mathrm{E}-04$ & & & \\
\hline Np-239 & $1.9 \mathrm{E}-04$ & $1.3 \mathrm{E}-04$ & & & \\
\hline $\mathrm{Np}-240$ & $1.1 \mathrm{E}-05$ & $8.2 \mathrm{E}-06$ & & & \\
\hline $\mathrm{Np}-240 \mathrm{~m}$ & $9.2 \mathrm{E}-08$ & $6.8 \mathrm{E}-08$ & & & \\
\hline Pu-236 & $3.1 \mathrm{E}+\infty$ & $2.2 \mathrm{E}+00$ & & & \\
\hline Pu-238 & $1.2 \mathrm{E}+01$ & $8.4 \mathrm{E}+00$ & & & \\
\hline Pu-239 & $1.3 \mathrm{E}+01$ & $9.0 \mathrm{E}+00$ & & & \\
\hline Pu-240 & $1.3 E+01$ & $9.0 \mathrm{E}+00$ & & & \\
\hline $\mathrm{Pu}-241$ & $2.0 \mathrm{E}-01$ & $1.4 \mathrm{E}-01$ & & & \\
\hline Pu-242 & $1.2 \mathrm{E}+01$ & $8.6 \mathrm{E}+00$ & & & \\
\hline Pu-243 & $6.8 \mathrm{E}-06$ & $4.9 \mathrm{E}-06$ & & & \\
\hline Pu-244 & $1.2 \mathrm{E}+01$ & $8.5 \mathrm{E}+\infty 0$ & & & \\
\hline Am-241 & $2.0 \mathrm{E}+01$ & $1.4 \mathrm{E}+01$ & & & \\
\hline Am-242 & $1.9 \mathrm{E}-03$ & $1.3 \mathrm{E}-03$ & & & \\
\hline$A m-242 m$ & $1.9 \mathrm{E}+01$ & $1.3 \mathrm{E}+01$ & & & \\
\hline Am-243 & $2.0 \mathrm{E}+01$ & $1.4 \mathrm{E}+01$ & & & \\
\hline $\mathrm{Cm}-242$ & $6.3 \mathrm{E}-01$ & $4.5 \mathrm{E}-01$ & & & \\
\hline $\mathrm{Cm}-243$ & $1.3 \mathrm{E}+01$ & $9.3 E+00$ & & & \\
\hline $\mathrm{Cm}-244$ & $1.0 \mathrm{E}+01$ & $7.3 E+00$ & & & \\
\hline $\mathrm{Cm}-245$ & $2.0 \mathrm{E}+01$ & $1.4 \mathrm{E}+01$ & & & \\
\hline $\mathrm{Cm}-246$ & $2.0 \mathrm{E}+01$ & $1.4 \mathrm{E}+01$ & & & \\
\hline $\mathrm{Cm}-247$ & $1.9 \mathrm{E}+01$ & $1.3 \mathrm{E}+01$ & & & \\
\hline $\mathrm{Cm}-248$ & $7.3 E+01$ & $5.2 \mathrm{E}+01$ & & & \\
\hline Cf-252 & $5.5 \mathrm{E}+00$ & $3.9 \mathrm{E}+00$ & & & \\
\hline
\end{tabular}

$=$ The effective release height, $x$, is in meters.

b CAP88-PC Ver. 1.0 does not calculate doses for Be-10, Se-79, Cd-113, Cd-113m, and Gd-152. Recommend substituting the unit dose factors of the nuclides in parentheses.

c Extremely short lived nuclide, resulting in an offsite dose of zero.

- Short lived radon nuclides only provide the mechanism to circumvent the emission control device. The unit dose factors for the recommended progeny should be used instead. However, $1 \mathrm{ci}$ of $\mathrm{Rn}$ does not equal $1 \mathrm{ci}$ of Pb. Multiply the activity of Rn-219 by 0.0018 to get the activity of $\mathrm{Pb}-211$. Multiply the activity of $\mathrm{Rn}-220$ by 0.0014 to get the activity of $\mathrm{Pb}-212$. 
Table 4-9. 200 East Area--CAP88-PC Unit Dose Factors by Effective Release Height ${ }^{\mathrm{a}}$ (mrem/Ci).

\begin{tabular}{|c|c|c|c|c|c|}
\hline \multirow{2}{*}{ Nuclide } & \multicolumn{2}{|c|}{$\begin{array}{c}\text { Effective } \\
\text { Release Height }\end{array}$} & \multirow{2}{*}{ Nuclide } & \multicolumn{2}{|c|}{$\begin{array}{c}\text { Effective } \\
\text { Release Height }\end{array}$} \\
\hline & $\mathrm{H}_{\text {eff }}<40 \mathrm{~m}$ & $\mathrm{H}_{\text {eff }} \geq 40 \mathrm{~m}$ & & $\mathrm{H}_{\mathrm{eff}}<40 \mathrm{~m}$ & $\mathrm{H}_{\text {eff } 2} 40 \mathrm{~m}$ \\
\hline $\mathrm{H}-3$ & $2.5 \mathrm{E}-05$ & $1.4 \mathrm{E}-05$ & $\mathrm{Kr}-85$ & $5.5 \mathrm{E}-08$ & $3.1 \mathrm{E}-08$ \\
\hline $\mathrm{Be}-7$ & $2.0 \mathrm{E}-04$ & $1.3 \mathrm{E}-04$ & $\mathrm{Kr}-85 \mathrm{~m}$ & $2.0 \mathrm{E}-06$ & $1.1 \mathrm{E}-06$ \\
\hline $\mathrm{Be}-10^{\mathrm{b}}$ & (Sr-90) & $(\mathrm{Sr}-90)$ & $\mathrm{Kr}-87$ & $6.1 \mathrm{E}-06$ & $3.7 \mathrm{E}-06$ \\
\hline $\mathrm{C}-11$ & $1.0 \mathrm{E}-06$ & $7.7 \mathrm{E}-07$ & $\mathrm{Kr}-88$ & $2.4 \mathrm{E}-05$ & $1.4 \mathrm{E}-05$ \\
\hline C-14 & $1.9 \mathrm{E}-03$ & $1.1 \mathrm{E}-03$ & $\mathrm{Kr}-89$ & $4.0 \mathrm{E}-11$ & $2.9 \mathrm{E}-11$ \\
\hline $\mathrm{C}-15^{\mathrm{c}}$ & 0.0 & 0.0 & $\mathrm{Kr}-90^{\mathrm{c}}$ & 0.0 & 0.0 \\
\hline $\mathrm{N}-13$ & $9.9 \mathrm{E}-08$ & $7.9 \mathrm{E}-08$ & $\mathrm{Rb}-86$ & $2.9 \mathrm{E}-\mathrm{C} 3$ & $1.9 \mathrm{E}-03$ \\
\hline $0-15$ & $1.9 \mathrm{E}-14$ & $1.5 \mathrm{E}-14$ & $\mathrm{Rb}-87$ & $9.4 \mathrm{E}-03$ & $6.4 \mathrm{E}-03$ \\
\hline F-18 & $1.0 \mathrm{E}-05$ & $7.0 \mathrm{E}-06$ & $\mathrm{Rb}-88$ & $6.1 \mathrm{E}-07$ & $4.6 \mathrm{E}-07$ \\
\hline $\mathrm{Na}-22$ & $1.3 \mathrm{E}-01$ & 8.7E-02 & $\mathrm{Rb}-89$ & $1.1 \mathrm{E}-06$ & $8.2 \mathrm{E}-07$ \\
\hline $\mathrm{Na}-24$ & $2.1 \mathrm{E}-04$ & $1.4 \mathrm{E}-04$ & $\mathrm{Rb}-90$ & $3.3 \mathrm{E}-12$ & $2.6 \mathrm{E}-12$ \\
\hline P-32 & $2.9 \mathrm{E}-03$ & $2.0 \mathrm{E}-03$ & $\mathrm{Rb}-90 \mathrm{~m}$ & $1.7 \mathrm{E}-09$ & $1.3 \mathrm{E}-09$ \\
\hline S-35 & 4.1E-04 & $2.8 \mathrm{E}-04$ & $\mathrm{Sr}-89$ & $1.7 \mathrm{E}-03$ & $1.2 \mathrm{E}-03$ \\
\hline $\mathrm{Ar}-41$ & $1.2 \mathrm{E}-05$ & $6.7 \mathrm{E}-06$ & Sr-90 & 1.1E-01 & $7.6 \mathrm{E}-02$ \\
\hline $\mathrm{K}-40$ & $1.2 \mathrm{E}-01$ & $8.4 \mathrm{E}-02$ & Sr-91 & 4.4E-05 & $2.9 \mathrm{E}-05$ \\
\hline $\mathrm{Ca}-41$ & $1.0 \mathrm{E}-05$ & $6.9 \mathrm{E}-06$ & Sr-92 & $2.6 \mathrm{E}-05$ & $1.8 \mathrm{E}-05$ \\
\hline Sc-46 & $1.2 \mathrm{E}-02$ & $8.2 \mathrm{E}-03$ & Y-90 & $3.4 \mathrm{E}-04$ & $2.3 \mathrm{E}-04$ \\
\hline $\mathrm{Cr}-51$ & 8.7E-05 & $5.9 \mathrm{E}-05$ & $Y-90 \mathrm{~m}$ & $9.3 \mathrm{E}-06$ & $6.2 \mathrm{E}-06$ \\
\hline $\mathrm{Mn}-54$ & $1.6 \mathrm{E}-02$ & $1.1 \mathrm{E}-02$ & $Y-91$ & $2.5 \mathrm{E}-03$ & $1.7 \mathrm{E}-03$ \\
\hline $\mathrm{Mn}-56$ & $2.9 \mathrm{E}-05$ & $1.9 \mathrm{E}-05$ & $\mathrm{Y}-91 \mathrm{~m}$ & $2.6 \mathrm{E}-06$ & $1.8 \mathrm{E}-06$ \\
\hline $\mathrm{Fe}-55$ & $2.3 \mathrm{E}-04$ & $1.6 \mathrm{E}-04$ & $Y-92$ & $2.3 \mathrm{E}-05$ & $1.5 \mathrm{E}-05$ \\
\hline $\mathrm{Fe}-59$ & 4.7E-03 & $3.2 \mathrm{E}-03$ & Y-93 & $5.8 \mathrm{E}-05$ & $3.8 \mathrm{E}-05$ \\
\hline $\mathrm{Co}-57$ & $2.8 \mathrm{E}-03$ & $1.9 \mathrm{E}-03$ & Zr-93 & $1.3 \mathrm{E}-03$ & $8.4 \mathrm{E}-04$ \\
\hline $\mathrm{Co}-58$ & $5.5 \mathrm{E}-03$ & $3.7 \mathrm{E}-03$ & $\mathrm{Zr}-95$ & $3.9 \mathrm{E}-03$ & $2.6 \mathrm{E}-03$ \\
\hline $\mathrm{Co}-60$ & $2.5 \mathrm{E}-01$ & $1.7 \mathrm{E}-01$ & $\mathrm{Nb}-93 \mathrm{~m}$ & $2.1 \mathrm{E}-03$ & $1.4 \mathrm{E}-03$ \\
\hline Ni-59 & $3.1 \mathrm{E}-04$ & 2.1E-04 & $\mathrm{Nb}-94$ & $8.1 \mathrm{E}-01$ & $5.5 \mathrm{E}-01$ \\
\hline $\mathrm{Ni}-63$ & $2.6 \mathrm{E}-04$ & $1.8 \mathrm{E}-04$ & $\mathrm{Nb}-95$ & $4.8 \mathrm{E}-03$ & $3.2 \mathrm{E}-03$ \\
\hline $\mathrm{Ni}-65$ & $1.2 \mathrm{E}-05$ & $7.7 \mathrm{E}-06$ & $\mathrm{Nb}-95 \mathrm{~m}$ & $3.0 \mathrm{E}-04$ & $2.0 \mathrm{E}-04$ \\
\hline $\mathrm{Cu}-64$ & $1.5 \mathrm{E}-05$ & $1.0 \mathrm{E}-05$ & $\mathrm{Nb}-97$ & $5.7 \mathrm{E}-06$ & $3.9 \mathrm{E}-06$ \\
\hline $\mathrm{Zn}-65$ & 2.5E-02 & $1.7 \mathrm{E}-02$ & $\mathrm{Nb}-97 \mathrm{~m}$ & $3.4 \mathrm{E}-23$ & $2.8 \mathrm{E}-23$. \\
\hline $\mathrm{Zn}-69 \mathrm{~m}$ & $4.1 \mathrm{E}-05$ & $2.7 \mathrm{E}-05$ & Mo-93 & $2.8 \mathrm{E}-03$ & $1.9 \mathrm{E}-03$ \\
\hline $\mathrm{Zn}-69$ & $4.9 \mathrm{E}-07$ & $3.4 \mathrm{E}-07$ & Mo-99 & $1.7 \mathrm{E}-04$ & $1.2 \mathrm{E}-04$ \\
\hline $\mathrm{Ga}-67$ & $6.1 \mathrm{E}-05$ & 4.1E-05 & Tc-97 & $5.7 \mathrm{E}-03$ & $3.9 \mathrm{E}-03$ \\
\hline As-76 & $1.4 \mathrm{E}-04$ & $8.9 \mathrm{E}-05$ & Tc-99 & $2.3 \mathrm{E}-02$ & $1.5 \mathrm{E}-02$ \\
\hline $\mathrm{Se}-79^{\mathrm{b}}$ & (Pu-241) & (Pu-241) & $\mathrm{Tc}-99 \mathrm{~m}$ & $3.8 \mathrm{E}-06$ & $2.5 \mathrm{E}-06$ \\
\hline $\mathrm{Br}-82$ & $3.2 \mathrm{E}-04$ & $2.2 \mathrm{E}-04$ & Tc-101 & $1.2 \mathrm{E}-07$ & $9.3 \mathrm{E}-08$ \\
\hline $\mathrm{Br}-83$ & $8.7 \mathrm{E}-08$ & $5.8 \mathrm{E}-08$ & Ru-97 & $6.6 \mathrm{E}-05$ & 4.5E-05 \\
\hline Br-84 & $4.6 \mathrm{E}-06$ & $3.3 \mathrm{E}-06$ & Ru-103 & $1.8 \mathrm{E}-03$ & $1.2 \mathrm{E}-03$ \\
\hline $\mathrm{Br}-85$ & $3.2 \mathrm{E}-13$ & $2.6 \mathrm{E}-13$ & Ru-105 & $2.5 \mathrm{E}-05$ & $1.7 \mathrm{E}-05$ \\
\hline $\mathrm{Kr}-83 \mathrm{~m}$ & 2.1E-09 & $1.2 \mathrm{E}-09$ & Ru-106 & $1.6 \mathrm{E}-02$ & 1.0E-02 \\
\hline
\end{tabular}


Table 4-9. 200 East Area--CAP88-PC Unit Dose Factors by Effective Release Height ${ }^{\mathrm{a}}$ (mrem/Ci).

\begin{tabular}{|c|c|c|c|c|c|}
\hline \multirow{2}{*}{ Nuclide } & \multicolumn{2}{|c|}{$\begin{array}{c}\text { Effective } \\
\text { Release Height }\end{array}$} & \multirow{2}{*}{ Nuclide } & \multicolumn{2}{|c|}{$\begin{array}{c}\text { Effective } \\
\text { Release Height }\end{array}$} \\
\hline & $\mathrm{H}_{\mathrm{eff}}<40 \mathrm{~m}$ & $\mathrm{H}_{\text {eff } Z} 40 \mathrm{~m}$ & & $\mathrm{H}_{\mathrm{eff}}<40 \mathrm{~m}$ & $\mathrm{H}_{\text {eff }} \geq 40 \mathrm{~m}$ \\
\hline Rh-103m & $6.3 \mathrm{E}-08$ & $4.3 \mathrm{E}-08$ & I-129 & $2.0 \mathrm{E}-01$ & $4.8 \mathrm{E}-01$ \\
\hline Rh-105 & $4.8 \mathrm{E}-05$ & $3.2 \mathrm{E}-05$ & $\mathrm{I}-130$ & 7.1E-05 & $1.7 \mathrm{E}-04$ \\
\hline Rh-105m & $3.1 \mathrm{E}-30$ & $2.5 \mathrm{E}-30$ & I-131 & $6.4 \mathrm{E}-03$ & $1.6 \mathrm{E}-02$ \\
\hline $\mathrm{Rh}-106^{\mathrm{c}}$ & 0.0 & 0.0 & $\mathrm{I}-132$ & $1.0 \mathrm{E}-05$ & $2.4 \mathrm{E}-05$ \\
\hline Pd-107 & $3.7 \mathrm{E}-04$ & $2.5 \mathrm{E}-04$ & I-133 & $7.3 \mathrm{E}-05$ & $1.8 \mathrm{E}-04$ \\
\hline Pd-109 & $3.5 \mathrm{E}-05$ & $2.3 \mathrm{E}-05$ & I-134 & $3.0 \mathrm{E}-06$ & $6.6 \mathrm{E}-06$ \\
\hline $\mathrm{Ag}-109 \mathrm{~m}$ & 3.3E-35 & $2.6 \mathrm{E}-35$ & I-135 & 2.3E-05 & $5.6 \mathrm{E}-05$ \\
\hline $\mathrm{Ag}-110^{\mathrm{c}}$ & 0.0 & 0.0 & $\mathrm{Xe}-122$ & $1.0 \mathrm{E}-06$ & $5.8 \mathrm{E}-07$ \\
\hline $\mathrm{Ag}-110 \mathrm{~m}$ & $4.8 \mathrm{E}-02$ & 3.3E-02 & $\mathrm{Xe}-123$ & $6.0 \mathrm{E}-06$ & $3.5 \mathrm{E}-06$ \\
\hline Ag-111 & $1.0 \mathrm{E}-03$ & $6.8 \mathrm{E}-04$ & $\mathrm{Xe}-125$ & $3.7 \mathrm{E}-06$ & 2.1E-06 \\
\hline $\mathrm{Cd}-113^{\mathrm{b}}$ & $(\mathrm{Pu}-241)$ & (Pu-241) & $\mathrm{Xe}-127$ & $4.2 \mathrm{E}-06$ & $2.4 \mathrm{E}-06$ \\
\hline $\mathrm{Cd}-113 \mathrm{~m}^{\mathrm{b}}$ & (Pu-241) & (Pu-241) & $\mathrm{Xe}-131 \mathrm{~m}$ & $1.5 \mathrm{E}-07$ & $8.7 \mathrm{E}-08$ \\
\hline Cd-115 & $1.9 \mathrm{E}-04$ & $1.3 E-04$ & Xe-133 & $5.6 \mathrm{E}-07$ & $3.2 \mathrm{E}-07$ \\
\hline $\mathrm{Cd}-115 \mathrm{~m}$ & $3.6 \mathrm{E}-03$ & $2.4 \mathrm{E}-03$ & $\mathrm{Xe}-133 \mathrm{~m}$ & $4.8 \mathrm{E}-07$ & $2.7 \mathrm{E}-07$ \\
\hline In-113m & $2.9 \mathrm{E}-06$ & $1.9 \mathrm{E}-06$ & $\mathrm{Xe}-135$ & $3.5 \mathrm{E}-06$ & $2.0 \mathrm{E}-06$ \\
\hline In-115 & $6.0 \mathrm{E}-02$ & $4.0 \mathrm{E}-02$ & $\mathrm{Xe}-135 \mathrm{~m}$ & $2.2 \mathrm{E}-07$ & $1.5 \mathrm{E}-07$ \\
\hline In-115m & $5.9 \mathrm{E}-06$ & $3.9 \mathrm{E}-06$ & $\mathrm{Xe}-137$ & 3.3E-11 & $2.5 \mathrm{E}-11$ \\
\hline $\mathrm{Sn}-113$ & $2.2 \mathrm{E}-03$ & $1.5 \mathrm{E}-03$ & $\mathrm{Xe}-138$ & $5.0 \mathrm{E}-07$ & $3.5 \mathrm{E}-07$ \\
\hline $\mathrm{Sn}-123$ & $5.1 \mathrm{E}-05$ & $3.5 \mathrm{E}-05$ & Cs-134 & $1.0 \mathrm{E}-01$ & $6.8 \mathrm{E}-02$ \\
\hline Sn-125 & $2.4 \mathrm{E}-03$ & $1.6 \mathrm{E}-03$ & Cs-134m & $9.8 \mathrm{E}-07$ & $6.5 \mathrm{E}-07$ \\
\hline Sn-126 & $4.7 \mathrm{E}-02$ & $3.2 \mathrm{E}-02$ & Cs-135 & $4.4 \mathrm{E}-03$ & $3.0 \mathrm{E}-03$ \\
\hline $\mathrm{Sb}-124$ & $8.6 \mathrm{E}-03$ & $5.8 \mathrm{E}-03$ & Cs-136 & $3.7 \mathrm{E}-03$ & $2.5 \mathrm{E}-03$ \\
\hline $\mathrm{Sb}-125$ & 2.6E-02 & $1.8 \mathrm{E}-02$ & Cs-137 & $2.7 \mathrm{E}-02$ & $1.8 \mathrm{E}-02$ \\
\hline $\mathrm{Sb}-126$ & 3.3E-03 & $2.2 \mathrm{E}-03$ & Cs-138 & $6.4 \mathrm{E}-06$ & $4.6 \mathrm{E}-06$ \\
\hline $\mathrm{Sb}-126 \mathrm{~b}$ & $1.4 \mathrm{E}-06$ & $1.0 \mathrm{E}-06$ & Cs-139 & $2.4 \mathrm{E}-08$ & $1.9 \mathrm{E}-08$ \\
\hline $\mathrm{Sb}-127$ & $4.7 \mathrm{E}-04$ & $3.1 \mathrm{E}-04$ & $\mathrm{Ba}-133$ & $7.5 \mathrm{E}-02$ & $5.1 \mathrm{E}-02$ \\
\hline $\mathrm{Te}-125 \mathrm{~m}$ & $1.0 \mathrm{E}-03$ & $6.9 \mathrm{E}-04$ & $\mathrm{Ba}-133 \mathrm{~m}$ & $3.1 \mathrm{E}-05$ & $2.1 \mathrm{E}-05$ \\
\hline Te-127 & $8.4 \mathrm{E}-06$ & $5.5 \mathrm{E}-06$ & Ba-137m & $5.3 \mathrm{E}-13$ & $4.3 \mathrm{E}-13$ \\
\hline $\mathrm{Te}-127 \mathrm{~m}$ & $2.5 \mathrm{E}-03$ & $1.7 \mathrm{E}-03$ & Ba-139 & $2.2 \mathrm{E}-06$ & $1.5 \mathrm{E}-06$ \\
\hline $\mathrm{Te}-129$ & $1.5 \mathrm{E}-06$ & $1.0 \mathrm{E}-06$. & $\mathrm{Ba}-140$ & $1.1 \mathrm{E}-03$ & 7.2E-04 \\
\hline $\mathrm{Te}-129 \mathrm{~m}$ & $2.5 \mathrm{E}-03$ & $1.7 \mathrm{E}-03$ & $\mathrm{Ba}-141$ & $6.6 \mathrm{E}-07$ & $5.0 \mathrm{E}-07$ \\
\hline $\mathrm{Te}-131$ & $9.3 \mathrm{E}-07$ & $6.8 \mathrm{E}-07$ & $\mathrm{Ba}-142$ & $1.2 \mathrm{E}-07$ & $9.4 \mathrm{E}-08$ \\
\hline $\mathrm{Te}-131 \mathrm{~m}$ & $2.4 \mathrm{E}-04$ & $1.6 \mathrm{E}-04$ & $\mathrm{La}-140$ & $4.0 \mathrm{E}-04$ & $2.7 \mathrm{E}-04$ \\
\hline Te-132 & $4.1 \mathrm{E}-04$ & $2.7 \mathrm{E}-04$ & La-141 & $6.9 \mathrm{E}-07$ & $4.6 \mathrm{E}-07$ \\
\hline $\mathrm{Te}-133$ & $2.2 \mathrm{E}-07$ & $1.7 \mathrm{E}-07$ & La-142 & $2.5 \mathrm{E}-05$ & $1.7 \mathrm{E}-05$ \\
\hline $\mathrm{Te}-133 \mathrm{~m}$ & $1.1 \mathrm{E}-05$ & $7.9 \mathrm{E}-06$ & Ce-141 & $7.5 \mathrm{E}-04$ & $5.1 \mathrm{E}-04$ \\
\hline $\mathrm{Te}-134$ & $3.1 \mathrm{E}-06$ & $2.2 \mathrm{E}-06$ & $\mathrm{Ce}-143$ & $1.2 \mathrm{E}-04$ & $7.8 \mathrm{E}-05$ \\
\hline I-122 & $9.9 \mathrm{E}-12$ & $1.8 \mathrm{E}-11$ & $\mathrm{Ce}-144$ & $1.2 \mathrm{E}-02$ & $8.1 \mathrm{E}-03$ \\
\hline I-123 & $6.5 \mathrm{E}-06$ & $1.6 \mathrm{E}-05$ & Pr-143 & $5.9 \mathrm{E}-04$ & $4.0 \mathrm{E}-04$ \\
\hline I-125 & $1.2 \mathrm{E}-02$ & $2.9 \mathrm{E}-02$ & Pr-144 & $1.1 \mathrm{E}-07$ & $7.9 \mathrm{E}-08$ \\
\hline
\end{tabular}


Table 4-9. 200 East Area--CAP88-PC Unit Dose Factors by Effective Release Height ${ }^{a}$ $(\mathrm{mrem} / \mathrm{Ci})$.

\begin{tabular}{|c|c|c|c|c|c|}
\hline \multirow{2}{*}{ Nuclide } & \multicolumn{2}{|c|}{$\begin{array}{c}\text { Effective } \\
\text { Release Height }\end{array}$} & \multirow{2}{*}{ Nuclide } & \multicolumn{2}{|c|}{$\begin{array}{c}\text { Effective } \\
\text { Release Height }\end{array}$} \\
\hline & $\mathrm{H}_{\text {eff }}<40 \mathrm{~m}$ & $\mathrm{H}_{\text {eff }} \geq 40 \mathrm{~m}$ & & $\mathrm{H}_{\mathrm{eff}}<40 \mathrm{~m}$ & $\mathrm{H}_{\text {eff }} \geq 40 \mathrm{~m}$ \\
\hline Pr-144m & $1.2 \mathrm{E}-09$ & $9.9 \mathrm{E}-10$ & Po-211 & 0.0 & 0.0 \\
\hline Nd-147 & $5.8 \mathrm{E}-04$ & $3.9 \mathrm{E}-04$ & Po- $212^{\mathrm{c}}$ & 0.0 & 0.0 \\
\hline Pm-147 & $1.1 \mathrm{E}-03$ & $7.0 \mathrm{E}-04$ & $\mathrm{P}_{0}-213^{\mathrm{c}}$ & 0.0 & 0.0 \\
\hline Pm-148 & $8.6 \mathrm{E}-04$ & $5.7 \mathrm{E}-04$ & Po- $214^{\mathrm{c}}$ & 0.0 & 0.0 \\
\hline $\mathrm{Pm}-148 \mathrm{~m}$ & $7.8 \mathrm{E}-03$ & $5.3 \mathrm{E}-03$ & Po- $215^{\mathrm{c}}$ & 0.0 & 0.0 \\
\hline Pm-149 & 1.1E-04 & $7.2 \mathrm{E}-05$ & Po- $216^{\mathrm{c}}$ & 0.0 & 0.0 \\
\hline Pm-151 & $2.9 \mathrm{E}-05$ & $1.9 \mathrm{E}-05$ & Po-218 & $9.7 \mathrm{E}-13$ & $7.8 \mathrm{E}-13$ \\
\hline Sm-147 & $1.7 \mathrm{E}+\infty$ & $1.1 \mathrm{E}+00$ & At $-217^{c}$ & 0.0 & 0.0 \\
\hline $\mathrm{Sm}-151$ & 7.5E-04 & $4.9 \mathrm{E}-04$ & Rn-219 & $(\mathrm{Pb}-211)$ & $(\mathrm{Pb}-211)$ \\
\hline $\mathrm{Sm}-153$ & $7.7 \mathrm{E}-05$ & $5.1 \mathrm{E}-05$ & $\mathrm{Rn}-220^{\mathrm{d}}$ & $(\mathrm{Pb}-212)$ & $(\mathrm{Pb}-212)$ \\
\hline Eu-152 & $2.4 \mathrm{E}-01$ & $1.7 \mathrm{E}-01$ & $\mathrm{Rn}-222$ & $6.9 \mathrm{E}-05$ & $3.9 \mathrm{E}-05$ \\
\hline $\mathrm{Eu}-152 \mathrm{~m}$ & $1.0 \mathrm{E}-05$ & $6.8 \mathrm{E}-06$ & Fr-221 & $8.8 \mathrm{E}-09$ & $7.2 \mathrm{E}-09$ \\
\hline Eu-154 & $2.0 \mathrm{E}-01$ & $1.3 \mathrm{E}-01$ & Fr-223 & $4.9 \mathrm{E}-06$ & $3.7 \mathrm{E}-06$ \\
\hline Eu-155 & $8.0 \mathrm{E}-03$ & $5.4 \mathrm{E}-03$ & $\mathrm{Ra}-223$ & $2.4 \mathrm{E}-01$ & $1.6 \mathrm{E}-01$ \\
\hline Eu-156 & $2.4 \mathrm{E}-03$ & $1.6 \mathrm{E}-03$ & $\mathrm{Ra}-224$ & $8.5 \mathrm{E}-02$ & $5.6 \mathrm{E}-02$ \\
\hline $\mathrm{Gd}-152^{\mathrm{b}}$ & $(\mathrm{Pu}-239)$ & (Pu-239) & $\mathrm{Ra}-225$ & $1.5 \mathrm{E}-01$ & $9.8 \mathrm{E}-02$ \\
\hline $\mathrm{Tb}-160$ & $6.4 \mathrm{E}-03$ & $4.3 \mathrm{E}-03$ & $\mathrm{Ra}-226$ & $4.6 \mathrm{E}-01$ & $3.1 \mathrm{E}-01$ \\
\hline Ho-166 & $8.9 \mathrm{E}-05$ & $5.8 \mathrm{E}-05$ & $\mathrm{Ra}-228$ & $1.9 \mathrm{E}-01$ & $1.3 \mathrm{E}-01$ \\
\hline Ho-166m & $8.0 \mathrm{E}-01$ & $5.4 \mathrm{E}-01$ & Ac-225 & $1.4 \mathrm{E}-01$ & $9.1 \mathrm{E}-02$ \\
\hline Hf-181 & $2.5 \mathrm{E}-03$ & $1.7 \mathrm{E}-03$ & Ac-227 & $1.5 \mathrm{E}+01$ & $9.5 \mathrm{E}+00$ \\
\hline W-181 & $4.5 \mathrm{E}-04$ & $3.1 \mathrm{E}-04$ & Ac- 228 & $1.6 \mathrm{E}-03$ & $1.0 \mathrm{E}-03$ \\
\hline W-185 & $6.0 \mathrm{E}-04$ & 4.1E-04 & Th-227 & $2.6 \mathrm{E}-01$ & $1.7 \mathrm{E}-01$ \\
\hline W-187 & $5.1 \mathrm{E}-05$ & $3.4 \mathrm{E}-05$ & Th-228 & $5.6 \mathrm{E}+00$ & $3.6 \mathrm{E}+\infty 0$ \\
\hline $\operatorname{Re}-187$ & $1.7 \mathrm{E}-05$ & $1.2 \mathrm{E}-05$ & Th-229 & $1.6 \mathrm{E}+01$ & $1.0 \mathrm{E}+01$ \\
\hline Ir-192 & $5.5 \mathrm{E}-03$ & $3.7 \mathrm{E}-03$ & Th-230 & $5.6 \mathrm{E}+\infty 0$ & $3.6 \mathrm{E}+\infty 0$ \\
\hline $\mathrm{Hg}-203$ & $3.4 \mathrm{E}-03$ & 2.3E-03 & Th-231 & $2.4 \mathrm{E}-05$ & $1.6 \mathrm{E}-05$ \\
\hline Tl-207 & $1.8 \mathrm{E}-11$ & $1.5 \mathrm{E}-11$ & Th-232 & $8.0 \mathrm{E}+00$ & $5.2 \mathrm{E}+00$ \\
\hline $\mathrm{Tl}-208$ & $4.2 \mathrm{E}-11$ & 3.4E-11 & Th-234 & $2.4 \mathrm{E}-03$ & $1.6 \mathrm{E}-03$ \\
\hline T1-209 & $1.7 \mathrm{E}-13$ & $1.3 \mathrm{E}-13$ & $\mathrm{~Pa}-231$ & $1.2 E+01$ & $7.5 \mathrm{E}+00$ \\
\hline $\mathrm{Pb}-209$ & $1.5 \mathrm{E}-06$ & $1.0 \mathrm{E}-06$ & $\mathrm{~Pa}-233$ & $1.0 \mathrm{E}-03$ & $7.0 \mathrm{E}-04$ \\
\hline $\mathrm{Pb}-210$ & $1.4 \mathrm{E}+00$ & $9.5 \mathrm{E}-01$ & $\mathrm{~Pa}-234$ & $6.9 \mathrm{E}-05$ & $4.6 \mathrm{E}-05$ \\
\hline $\mathrm{Pb}-211$ & $4.2 \mathrm{E}-05$ & $3.0 \mathrm{E}-05$ & $\mathrm{~Pa}-234 \mathrm{~m}$ & $2.4 \mathrm{E}-22$ & $1.9 \mathrm{E}-22$ \\
\hline $\mathrm{Pb}-212$ & 3.3E-03 & $2.1 \mathrm{E}-03$ & U-232 & $1.1 \mathrm{E}+01$ & $7.2 \mathrm{E}+00$ \\
\hline $\mathrm{Pb}-214$ & $3.0 \mathrm{E}-06$ & $2.2 \mathrm{E}-06$ & U-233 & $3.1 E+\infty 0$ & $2.0 \mathrm{E}+00$ \\
\hline $\mathrm{Bi}-210$ & $4.5 \mathrm{E}-03$ & $2.9 \mathrm{E}-03$ & U-234 & $3.1 E+\infty 0$ & $2.0 \mathrm{E}+00$ \\
\hline $\mathrm{Bi}-211$ & $8.1 \mathrm{E}-14$ & $6.5 \mathrm{E}-14$ & U-235 & $3.0 \mathrm{E}+\infty 0$ & $1.9 \mathrm{E}+00$ \\
\hline Bi-212 & $2.7 \mathrm{E}-04$ & $1.8 \mathrm{E}-04$ & U-236 & $2.9 \mathrm{E}+\infty 0$ & $1.9 \mathrm{E}+00$ \\
\hline$B i-213$ & $7.9 \mathrm{E}-06$ & $5.5 \mathrm{E}-06$ & U-237 & $3.1 \mathrm{E}-04$ & $2.1 \mathrm{E}-04$ \\
\hline Bi-214 & $2.0 \mathrm{E}-06$ & $1.5 \mathrm{E}-06$ & U-238 & $2.8 \mathrm{E}+\infty$ & $1.8 \mathrm{E}+00$ \\
\hline Po-210 & $5.2 \mathrm{E}-01$ & $3.5 \mathrm{E}-01$ & $\mathrm{U}-240$ & $5.1 \mathrm{E}-05$ & 3.3E-05 \\
\hline
\end{tabular}


HNF-3602, Vol. 1

Table 4-9. 200 East Area--CAP88-PC Unit Dose Factors by Effective Release Height ${ }^{\mathrm{a}}$ (mrem/Ci).

\begin{tabular}{|c|c|c|c|c|c|}
\hline \multirow{2}{*}{ Nuclide } & \multicolumn{2}{|c|}{$\begin{array}{c}\text { Effective } \\
\text { Release Height }\end{array}$} & \multirow{2}{*}{ Nuclide } & \multicolumn{2}{|c|}{$\begin{array}{c}\text { Effective } \\
\text { Release Height }\end{array}$} \\
\hline & $\mathrm{H}_{\mathrm{eff}}<40 \mathrm{~m}$ & $\mathrm{H}_{\text {eff }} \geq 40 \mathrm{~m}$ & & $\mathrm{H}_{\mathrm{eff}}<40 \mathrm{~m}$ & $\mathrm{H}_{\text {eff }} \geq 40 \mathrm{~m}$ \\
\hline Np-237 & $1.2 \mathrm{E}+01$ & $7.5 \mathrm{E}+00$ & & & \\
\hline $\mathrm{Np}-238$ & $7.9 \mathrm{E}-04$ & $5.2 \mathrm{E}-04$ & & & \\
\hline Np-239 & $1.2 \mathrm{E}-04$ & $8.0 \mathrm{E}-05$ & & & \\
\hline $\mathrm{Np}-240$ & $8.0 \mathrm{E}-06$ & $5.5 \mathrm{E}-06$ & & & \\
\hline $\mathrm{Np}-240 \mathrm{~m}$ & $8.2 \mathrm{E}-09$ & $6.6 \mathrm{E}-09$ & & & \\
\hline Pu-236 & $2.0 \mathrm{E}+00$ & $1.3 \mathrm{E}+00$ & & & \\
\hline Pu-238 & $7.6 \mathrm{E}+00$ & $5.0 \mathrm{E}+\infty 0$ & & & \\
\hline Pu-239 & $8.2 \mathrm{E}+00$ & $5.4 \mathrm{E}+00$ & & & \\
\hline $\mathrm{Pu}-240$ & $8.2 \mathrm{E}+00$ & $5.3 \mathrm{E}+00$ & & & \\
\hline Pu-241 & $1.3 \mathrm{E}-01$ & $8.4 \mathrm{E}-02$ & & & \\
\hline Pu-242 & $7.8 \mathrm{E}+00$ & $5.1 \mathrm{E}+\infty 0$ & & & \\
\hline Pu-243 & 4.4E-06 & $2.9 \mathrm{E}-06$ & & & \\
\hline $\mathrm{Pu}-244$ & $7.8 \mathrm{E}+00$ & $5.1 \mathrm{E}+00$ & & & \\
\hline Am-241 & $1.3 \mathrm{E}+01$ & $8.2 \mathrm{E}+00$ & & & \\
\hline Am-242 & $1.2 \mathrm{E}-03$ & $7.9 \mathrm{E}-04$ & & & \\
\hline $\mathrm{Am}-242 \mathrm{~m}$ & $1.2 \mathrm{E}+01$ & $7.9 \mathrm{E}+00$ & & & \\
\hline Am-243 & 1.3E+01 & $8.2 \mathrm{E}+00$ & & & \\
\hline $\mathrm{Cm}-242$ & 4.1E-01 & $2.7 \mathrm{E}-01$ & & & \\
\hline $\mathrm{Cm}-243$ & $8.5 E+00$ & $5.5 E+00$ & & & \\
\hline $\mathrm{Cm}-244$ & $6.7 E+00$ & $4.3 \mathrm{E}+00$ & & & \\
\hline $\mathrm{Cm}-245$ & $1.3 \mathrm{E}+01$ & $8.5 \mathrm{E}+00$ & & & \\
\hline $\mathrm{Cm}-246$ & $1.3 \mathrm{E}+01$ & $8.4 \mathrm{E}+00$ & & & \\
\hline $\mathrm{Cm}-247$ & $1.2 \mathrm{E}+01$ & $7.8 \mathrm{E}+00$ & & & \\
\hline $\mathrm{Cm}-248$ & $4.7 \mathrm{E}+01$ & $3.1 \mathrm{E}+01$ & & & \\
\hline Cf-252 & $3.5 \mathrm{E}+00$ & $2.3 \mathrm{E}+00$ & & & \\
\hline
\end{tabular}

2 The effective release height, $x$, is in meters.

b CAP88-PC Ver. 1.0 does not calcuiate doses for Be-10, Se-79, Cd-113, Cd-113m, and Gd-152. Recommend substituting the unit dose factors of the nuclides in parentheses.

- Extremely short lived nuclide, resulting in an offsite dose of zero.

d Short lived radon nuclides only provide the mechanism to circumvent the emission control device. The unit dose factors for the recommended progeny should be used instead. However, $1 \mathrm{ci}$ of $\mathrm{Rn}$ does not equal $1 \mathrm{ci}$ of $\mathrm{Pb}$. Multiply the activity of Rn-219 by 0.0018 to get the activity of $\mathrm{Pb}-211$. Multiply the activity of $\mathrm{Rn}-220$ by 0.0014 to get the activity of $\mathrm{Pb}-212$. 
Table 4-10. 200 West Area--CAP88-PC Unit Dose Factors by Effective Release Height $^{\mathrm{a}}(\mathrm{mrem} / \mathrm{Ci})$.

\begin{tabular}{|c|c|c|c|c|c|}
\hline \multirow{2}{*}{ Nuclide } & \multicolumn{2}{|c|}{$\begin{array}{c}\text { Effective } \\
\text { Release Height }\end{array}$} & \multirow{2}{*}{ Nuclide } & \multicolumn{2}{|c|}{$\begin{array}{c}\text { Effective } \\
\text { Release Height }\end{array}$} \\
\hline & $\mathrm{H}_{\text {eff }}<40 \mathrm{~m}$ & $\mathrm{H}_{\mathrm{eff}} \geq 40 \mathrm{~m}$ & & $\mathrm{H}_{\text {eff }}<40 \mathrm{~m}$ & $\mathrm{H}_{\text {eff }} \geq 40 \mathrm{~m}$ \\
\hline H-3 & $2.5 \mathrm{E}-05$ & $1.4 \mathrm{E}-05$ & $\mathrm{Kr}-85$ & $5.6 \mathrm{E}-08$ & $3.0 \mathrm{E}-08$ \\
\hline $\mathrm{Be}-7$ & $1.5 \mathrm{E}-04$ & $1.2 \mathrm{E}-04$ & $\mathrm{Kr}-85 \mathrm{~m}$ & $1.5 \mathrm{E}-06$ & $8.7 \mathrm{E}-07$ \\
\hline $\mathrm{Be}-10^{\mathrm{b}}$ & $(\mathrm{Sr}-90)$ & $(\mathrm{Sr}-90)$ & $\mathrm{Kr}-87$ & $3.0 \mathrm{E}-06$ & $2.0 \mathrm{E}-06$ \\
\hline C-11 & $3.8 \mathrm{E}-07$ & $3.4 \mathrm{E}-07$ & $\mathrm{Kr}-88$ & $1.6 \mathrm{E}-05$ & $9.7 \mathrm{E}-06$ \\
\hline C-14 & $2.0 \mathrm{E}-03$ & $1.1 \mathrm{E}-03$ & $\mathrm{Kr}-89$ & $9.8 \mathrm{E}-12$ & 7.2E-12 \\
\hline $\mathrm{C}-15^{\mathrm{c}}$ & 0.0 & 0.0 & $\mathrm{Kr}-90^{\mathrm{c}}$ & 0.0 & 0.0 \\
\hline $\mathrm{N}-13$ & $3.6 \mathrm{E}-08$ & 3.2E-08 & $\mathrm{Rb}-86$ & $2.2 \mathrm{E}-\mathrm{C} 3$ & $1.7 \mathrm{E}-03$ \\
\hline $0-15$ & 2.2E-15 & $1.9 \mathrm{E}-15$ & $\mathrm{Rb}-87$ & $7.4 \mathrm{E}-03$ & $5.7 \mathrm{E}-03$ \\
\hline F-18 & $4.8 \mathrm{E}-06$ & $4.0 \mathrm{E}-06$ & $\mathrm{Rb}-88$ & 2.2E-07 & $2.0 \mathrm{E}-07$ \\
\hline $\mathrm{Na}-22$ & $1.0 \mathrm{E}-01$ & $7.7 \mathrm{E}-02$ & $\mathrm{Rb}-89$ & $4.0 \mathrm{E}-07$ & $3.5 \mathrm{E}-07$ \\
\hline $\mathrm{Na}-24$ & $1.5 \mathrm{E}-04$ & $1.2 \mathrm{E}-04$ & Rb-90 & $5.4 \mathrm{E}-13$ & $4.8 \mathrm{E}-13$ \\
\hline P-32 & $2.3 \mathrm{E}-03$ & $1.7 \mathrm{E}-03$ & $\mathrm{Rb}-90 \mathrm{~m}$ & $4.5 \mathrm{E}-10$ & $3.9 \mathrm{E}-10$ \\
\hline S-35 & $3.2 \mathrm{E}-04$ & $2.5 \mathrm{E}-04$ & Sr-89 & $1.3 \mathrm{E}-03$ & $1.0 \mathrm{E}-03$ \\
\hline $\mathrm{Ar}-41$ & $6.3 \mathrm{E}-06$ & 4.1E-06 & Sr-90 & $8.8 \mathrm{E}-02$ & $6.8 \mathrm{E}-02$ \\
\hline $\mathrm{K}-40$ & $9.6 \mathrm{E}-02$ & $7.4 \mathrm{E}-02$ & Sr-91 & $3.0 \mathrm{E}-05$ & $2.3 \mathrm{E}-05$ \\
\hline $\mathrm{Ca}-41$ & $8.0 \mathrm{E}-06$ & $6.1 \mathrm{E}-06$ & Sr-92 & $1.4 \mathrm{E}-05$ & $1.1 \mathrm{E}-05$ \\
\hline Sc-46 & $9.4 \mathrm{E}-03$ & 7.3E-03 & Y-90 & $2.6 \mathrm{E}-04$ & $2.0 \mathrm{E}-04$ \\
\hline Cr-51 & $6.8 \mathrm{E}-05$ & $5.3 \mathrm{E}-05$ & $\mathrm{Y}-90 \mathrm{~m}$ & $5.1 \mathrm{E}-06$ & $4.1 \mathrm{E}-06$ \\
\hline Mn-54 & $1.3 \mathrm{E}-02$ & $9.9 \mathrm{E}-03$ & $Y-91$ & $2.0 \mathrm{E}-03$ & $1.5 \mathrm{E}-03$ \\
\hline $\mathrm{Mn}-56$ & $1.5 \mathrm{E}-05$ & $1.2 \mathrm{E}-05$ & Y-91m & $9.7 \mathrm{E}-07$ & $8.6 \mathrm{E}-07$ \\
\hline Fe-55 & $1.8 \mathrm{E}-04$ & $1.4 \mathrm{E}-04$ & $Y-92$ & $1.3 \mathrm{E}-05$ & $1.0 \mathrm{E}-05$ \\
\hline $\mathrm{Fe}-59$ & 3.7E-03 & $2.8 \mathrm{E}-03$ & Y-93 & $4.0 \mathrm{E}-05$ & $3.0 \mathrm{E}-05$ \\
\hline $\mathrm{Co}-57$ & 2.2E-03 & $1.7 \mathrm{E}-03$ & $\mathrm{Zr}-93$ & $9.9 \mathrm{E}-04$ & $7.4 \mathrm{E}-04$ \\
\hline Co-58 & $4.3 \mathrm{E}-03$ & $3.3 \mathrm{E}-03$ & $\mathrm{Zr}-95$ & $3.0 \mathrm{E}-03$ & $2.3 \mathrm{E}-03$ \\
\hline Co- 60 & $1.9 \mathrm{E}-01$ & $1.5 \mathrm{E}-01$ & $\mathrm{Nb}-93 \mathrm{~m}$ & $1.6 \mathrm{E}-03$ & $1.2 \mathrm{E}-03$ \\
\hline $\mathrm{Ni}-59$ & $2.4 \mathrm{E}-04$ & $1.9 \mathrm{E}-04$ & $\mathrm{Nb}-94$ & $6.3 \mathrm{E}-01$ & $4.9 \mathrm{E}-01$ \\
\hline $\mathrm{Ni}-63$ & $2.0 \mathrm{E}-04$ & $1.6 \mathrm{E}-04$ & $\mathrm{Nb}-95$ & $3.7 \mathrm{E}-03$ & $2.9 \mathrm{E}-03$ \\
\hline $\mathrm{Ni}-65$ & $5.8 \mathrm{E}-06$ & $4.7 \mathrm{E}-06$ & $\mathrm{Nb}-95 \mathrm{~m}$ & $2.3 \mathrm{E}-04$ & $1.8 \mathrm{E}-04$ \\
\hline $\mathrm{Cu}-64$ & $1.1 \mathrm{E}-05$ & 8.1E-06 & $\mathrm{Nb}-97$ & $2.3 \mathrm{E}-06$ & $2.0 \mathrm{E}-06$ \\
\hline Zn-65 & $1.9 \mathrm{E}-02$ & $1.5 \mathrm{E}-02$ & $\mathrm{Nb}-97 \mathrm{~m}$ & $6.9 \mathrm{E}-25$ & $6.0 \mathrm{E}-25$ \\
\hline $\mathrm{Zn}-69 \mathrm{~m}$ & $2.9 \mathrm{E}-05$ & $2.2 \mathrm{E}-05$ & Mo-93 & $2.2 \mathrm{E}-03$ & $1.7 \mathrm{E}-03$ \\
\hline $\mathrm{Zn}-69$ & $1.9 \mathrm{E}-07$ & $1.6 \mathrm{E}-07$ & Mo-99 & $1.3 \mathrm{E}-04$ & $1.0 \mathrm{E}-04$ \\
\hline $\mathrm{Ga}-67$ & 4.7E-05 & $3.6 \mathrm{E}-05$ & Tc-97 & $4.5 \mathrm{E}-03$ & $3.5 \mathrm{E}-03$ \\
\hline As-76 & $1.0 \mathrm{E}-04$ & 7.6E-05 & Tc-99 & $1.8 \mathrm{E}-02$ & $1.4 \mathrm{E}-02$ \\
\hline $\mathrm{Se}-79^{\mathrm{b}}$ & (Pu-241) & (Pu-241) & Tc-99m & 2.4E-06 & $1.9 \mathrm{E}-06$ \\
\hline $\mathrm{Br}-82$ & $2.4 \mathrm{E}-04$ & $1.9 \mathrm{E}-04$ & Tc-101 & $4.5 \mathrm{E}-08$ & 4.0E-08 \\
\hline $\mathrm{Br}-83$ & $4.4 \mathrm{E}-08$ & $3.6 \mathrm{E}-08$ & Ru-97 & $5.1 \mathrm{E}-05$ & $3.9 \mathrm{E}-05$ \\
\hline $\mathrm{Br}-84$ & $1.7 \mathrm{E}-06$ & $1.5 \mathrm{E}-06$ & Ru-103 & $1.4 \mathrm{E}-03$ & $1.1 \mathrm{E}-03$ \\
\hline $\mathrm{Br}-85$ & $6.0 \mathrm{E}-14$ & $5.3 \mathrm{E}-14$ & Ru-105 & $1.5 \mathrm{E}-05$ & $1.2 \mathrm{E}-05$ \\
\hline $\mathrm{Kr}-83 \mathrm{~m}$ & 1.1E-09 & 7.4E-10 & Ru-106 & $1.2 \mathrm{E}-02$ & $9.1 \mathrm{E}-03$ \\
\hline
\end{tabular}


HNF-3602, Vol. 1

Table 4-10. 200 West Area--CAP88-PC Unit Dose Factors by Effective Release Height $^{a}$ (mrem/Ci).

\begin{tabular}{|c|c|c|c|c|c|}
\hline \multirow{2}{*}{ Nuclide } & \multicolumn{2}{|c|}{$\begin{array}{c}\text { Effective } \\
\text { Release Height }\end{array}$} & \multirow{2}{*}{ Nuclide } & \multicolumn{2}{|c|}{$\begin{array}{c}\text { Effective } \\
\text { Release Height }\end{array}$} \\
\hline & $\mathrm{H}_{\mathrm{eff}}<40 \mathrm{~m}$ & $\mathrm{H}_{\text {eff }} \geq 40 \mathrm{~m}$ & & $\mathrm{H}_{\mathrm{eff}}<40 \mathrm{~m}$ & $\mathrm{H}_{\text {eff } \geq} 40 \mathrm{~m}$ \\
\hline Rh-103m & $2.4 \mathrm{E}-08$ & $2.1 \mathrm{E}-08$ & I-129 & $7.6 \mathrm{E}-02$ & $1.8 \mathrm{E}-01$ \\
\hline Rh-105 & $3.6 \mathrm{E}-05$ & $2.8 \mathrm{E}-05$ & I-130 & $2.7 \mathrm{E}-05$ & $6.0 \mathrm{E}-05$ \\
\hline Rh-105m & 7.7E-34 & $6.8 \mathrm{E}-34$ & I-131 & $2.5 \mathrm{E}-03$ & $5.8 \mathrm{E}-03$ \\
\hline Rh-106 & 0.0 & 0.0 & $\mathrm{I}-132$ & $3.5 \mathrm{E}-06$ & $6.8 \mathrm{E}-06$ \\
\hline Pd-107 & $2.9 \mathrm{E}-04$ & 2.2E-04 & I-133 & $2.8 \mathrm{E}-05$ & $6.4 \mathrm{E}-05$ \\
\hline Pd-109 & $2.5 \mathrm{E}-05$ & $1.9 \mathrm{E}-05$ & I-134 & $9.6 \mathrm{E}-07$ & $1.7 \mathrm{E}-06$ \\
\hline $\mathrm{Ag}-109 \mathrm{~m}$ & $1.1 \mathrm{E}-37$ & $9.6 \mathrm{E}-38$ & I- 135 & $8.5 \mathrm{E}-06$ & $1.9 \mathrm{E}-05$ \\
\hline $\mathrm{Ag}-110^{\mathrm{c}}$ & 0.0 & 0.0 & $\mathrm{Xe}-122$ & $9.9 \mathrm{E}-07$ & $5.4 \mathrm{E}-07$ \\
\hline $\mathrm{Ag}-110 \mathrm{~m}$ & $3.8 \mathrm{E}-02$ & $2.9 \mathrm{E}-02$ & $\mathrm{Xe}-123$ & $3.5 \mathrm{E}-06$ & $2.2 \mathrm{E}-06$ \\
\hline Ag-111 & $7.8 \mathrm{E}-04$ & $6.0 \mathrm{E}-04$ & $\mathrm{Xe}-125$ & $3.5 \mathrm{E}-06$ & $1.9 \mathrm{E}-06$ \\
\hline $\mathrm{Cd}-113^{\mathrm{b}}$ & (Pu-241) & (Pu-24l) & $\mathrm{Xe}-127$ & $4.4 \mathrm{E}-06$ & $2.3 \mathrm{E}-06$ \\
\hline $\mathrm{Cd}-113 \mathrm{~m}^{\mathrm{b}}$ & (Pu-241) & (Pu-241) & $\mathrm{Xe}-131 \mathrm{~m}$ & $1.6 \mathrm{E}-07$ & $8.5 \mathrm{E}-08$ \\
\hline Cd-115 & $1.5 \mathrm{E}-04$ & $1.1 \mathrm{E}-04$ & $\mathrm{Xe}-133$ & $5.7 \mathrm{E}-07$ & $3.1 \mathrm{E}-07$ \\
\hline $\mathrm{Cd}-115 \mathrm{~m}$ & $2.8 \mathrm{E}-03$ & $2.1 \mathrm{E}-03$ & $\mathrm{Xe}-133 \mathrm{~m}$ & $4.9 \mathrm{E}-07$ & $2.6 \mathrm{E}-07$ \\
\hline In-113m & $1.3 \mathrm{E}-06$ & $1.1 \mathrm{E}-06$ & $\mathrm{Xe}-135$ & $3.1 \mathrm{E}-06$ & $1.7 \mathrm{E}-06$ \\
\hline In-115 & $4.7 \mathrm{E}-02$ & 3.6E-02 & $\mathrm{Xe}-135 \mathrm{~m}$ & $9.5 \mathrm{E}-08$ & $7.0 \mathrm{E}-08$ \\
\hline In-115m & $3.5 \mathrm{E}-06$ & $2.7 \mathrm{E}-06$ & $\mathrm{Xe}-137$ & $9.8 \mathrm{E}-12$ & $7.2 \mathrm{E}-12$ \\
\hline Sn-113 & $1.7 \mathrm{E}-03$ & $1.3 \mathrm{E}-03$ & Xe-138 & $2.2 \mathrm{E}-07$ & $1.6 \mathrm{E}-07$ \\
\hline Sn-123 & $4.0 \mathrm{E}-05$ & $3.1 \mathrm{E}-05$ & Cs-134 & $7.8 \mathrm{E}-02$ & $6.0 \mathrm{E}-02$ \\
\hline Sn-125 & $1.8 \mathrm{E}-03$ & $1.4 \mathrm{E}-03$ & $\mathrm{Cs}-134 \mathrm{~m}$ & $5.2 \mathrm{E}-07$ & $4.2 \mathrm{E}-07$ \\
\hline $\mathrm{Sn}-126$ & $3.7 \mathrm{E}-02$ & $2.8 \mathrm{E}-02$ & Cs-135 & $3.5 \mathrm{E}-03$ & $2.7 \mathrm{E}-03$ \\
\hline$S b-124$ & $6.7 \mathrm{E}-03$ & $5.2 \mathrm{E}-03$ & Cs-136 & $2.9 \mathrm{E}-03$ & $2.2 E-03$ \\
\hline $\mathrm{Sb}-125$ & $2.1 \mathrm{E}-02$ & $1.6 \mathrm{E}-02$ & Cs-137 & $2.1 \mathrm{E}-02$ & $1.6 \mathrm{E}-02$ \\
\hline Sb-126 & $2.6 \mathrm{E}-03$ & $2.0 \mathrm{E}-03$ & Cs- 138 & $2.3 \mathrm{E}-06$ & 2.1E-06 \\
\hline Sb-126b & $5.0 \mathrm{E}-07$ & 4.4E-07 & Cs-139 & $8.8 \mathrm{E}-09$ & $7.8 \mathrm{E}-09$ \\
\hline $\mathrm{Sb}-127$ & $3.6 \mathrm{E}-04$ & $2.7 \mathrm{E}-04$ & Ba-133 & $5.9 \mathrm{E}-02$ & $4.5 \mathrm{E}-02$ \\
\hline $\mathrm{Te}-125 \mathrm{~m}$ & $8.1 \mathrm{E}-04$ & $6.2 \mathrm{E}-04$ & $\mathrm{Ba}-133 \mathrm{~m}$ & 2.4E-05 & $1.8 \mathrm{E}-05$ \\
\hline Te-127 & $5.7 \mathrm{E}-06$ & 4.3E-06 & $\mathrm{Ba}-137 \mathrm{~m}$ & $8.6 \mathrm{E}-14$ & 7.5E-14 \\
\hline $\mathrm{Te}-127 \mathrm{~m}$ & $2.0 \mathrm{E}-03$ & $1.5 \mathrm{E}-03$ & Ba-139 & 9.2E-07 & $7.8 \mathrm{E}-07$ \\
\hline $\mathrm{Te}-129$ & $6.1 \mathrm{E}-07$ & $5.3 \mathrm{E}-07$ & $\mathrm{Ba}-140$ & $8.3 \mathrm{E}-04$ & $6.4 \mathrm{E}-04$ \\
\hline $\mathrm{Te}-129 \mathrm{~m}$ & $2.0 \mathrm{E}-03$ & $1.5 \mathrm{E}-03$ & $\mathrm{Ba}-141$ & $2.4 \mathrm{E}-07$ & $2.2 \mathrm{E}-07$ \\
\hline $\mathrm{Te}-131$ & $3.4 \mathrm{E}-07$ & $3.0 \mathrm{E}-07$ & $\mathrm{Ba}-142$ & $4.4 \mathrm{E}-08$ & $3.9 \mathrm{E}-08$ \\
\hline $\mathrm{Te}-131 \mathrm{~m}$ & $1.8 \mathrm{E}-04$ & $1.3 \mathrm{E}-04$ & La-140 & $3.0 \mathrm{E}-04$ & $2.3 \mathrm{E}-04$ \\
\hline Te-132 & $3.2 \mathrm{E}-04$ & $2.4 \mathrm{E}-04$ & La-141 & 4.0E-07 & $3.2 \mathrm{E}-07$ \\
\hline Te-133 & $8.0 \mathrm{E}-08$ & $7.1 \mathrm{E}-08$ & La-142 & $1.1 \mathrm{E}-05$ & $9.2 \mathrm{E}-06$ \\
\hline $\mathrm{Te}-133 \mathrm{~m}$ & $4.4 \mathrm{E}-06$ & $3.8 \mathrm{E}-06$ & Ce-141 & $5.9 \mathrm{E}-04$ & $4.5 \mathrm{E}-04$ \\
\hline Te-134 & $1.1 \mathrm{E}-06$ & $1.0 \mathrm{E}-06$ & Ce-143 & $8.8 \mathrm{E}-05$ & $6.7 \mathrm{E}-05$ \\
\hline I-122 & $1.5 \mathrm{E}-12$ & $2.5 \mathrm{E}-12$ & Ce-144 & $9.6 \mathrm{E}-03$ & $7.2 \mathrm{E}-03$ \\
\hline I-123 & 2.4E-06 & $5.5 \mathrm{E}-06$ & Pr-143 & 4.6E-04 & $3.5 \mathrm{E}-04$ \\
\hline $\mathrm{I}-125$ & $4.5 \mathrm{E}-03$ & $1.1 \mathrm{E}-02$ & Pr-144 & $3.9 \mathrm{E}-08$ & $3.4 \mathrm{E}-08$ \\
\hline
\end{tabular}


HNF-3602, Vol. 1

Table 4-10. 200 West Area--CAP88-PC Unit Dose Factors by Effective Release Height ${ }^{\mathrm{a}}$ (mrem/Ci).

\begin{tabular}{|c|c|c|c|c|c|}
\hline \multirow{2}{*}{ Nuclide } & \multicolumn{2}{|c|}{$\begin{array}{c}\text { Effective } \\
\text { Release Height }\end{array}$} & \multirow{2}{*}{ Nuclide } & \multicolumn{2}{|c|}{$\begin{array}{c}\text { Effective } \\
\text { Release Height }\end{array}$} \\
\hline & $\mathrm{H}_{\mathrm{eff}}<40 \mathrm{~m}$ & $\mathrm{H}_{\text {eff }} \mathrm{40m}$ & & $\mathrm{H}_{\text {eff }}<40 \mathrm{~m}$ & $\mathrm{H}_{\text {eff }} \geq 40 \mathrm{~m}$ \\
\hline $\operatorname{Pr}-144 \mathrm{~m}$ & $4.2 \mathrm{E}-10$ & $3.7 \mathrm{E}-10$ & Po- $211^{\mathrm{c}}$ & 0.0 & 0.0 \\
\hline $\mathrm{Nd}-147$ & $4.5 \mathrm{E}-04$ & $3.4 \mathrm{E}-04$ & $\mathrm{Po}-212^{\mathrm{c}}$ & 0.0 & 0.0 \\
\hline Pm-147 & $8.4 \mathrm{E}-04$ & $6.2 \mathrm{E}-04$ & Po- $213^{c}$ & 0.0 & 0.0 \\
\hline $\mathrm{Pm}-148$ & $6.6 \mathrm{E}-04$ & $5.0 \mathrm{E}-04$ & Po-214 & 0.0 & 0.0 \\
\hline $\mathrm{Pm}-148 \mathrm{~m}$ & $6.1 \mathrm{E}-03$ & $4.7 \mathrm{E}-03$ & Po- $215^{\mathrm{c}}$ & 0.0 & 0.0 \\
\hline Pm-149 & $8.2 \mathrm{E}-05$ & $6.2 \mathrm{E}-05$ & Po-216 & 0.0 & 0.0 \\
\hline $\mathrm{Pm}-151$ & $2.1 \mathrm{E}-05$ & $1.7 \mathrm{E}-05$ & Po-218 & $1.9 \mathrm{E}-13$ & $1.7 \mathrm{E}-13$ \\
\hline Sm-147 & $1.3 \mathrm{E}+00$ & $9.6 \mathrm{E}-01$ & At $-217^{c}$ & 0.0 & 0.0 \\
\hline Sm-151 & $5.8 \mathrm{E}-04$ & $4.3 \mathrm{E}-04$ & Rn-219d & $(\mathrm{Pb}-211)$ & $(\mathrm{Pb}-211)$ \\
\hline $\mathrm{Sm}-153$ & $5.8 \mathrm{E}-05$ & $4.4 \mathrm{E}-05$ & $R n-220^{d}$ & $(\mathrm{~Pb}-212)$ & $(\mathrm{Pb}-212)$ \\
\hline Eu-152 & $1.9 \mathrm{E}-01$ & $1.5 \mathrm{E}-01$ & $\mathrm{Rn}-222$ & $7.0 \mathrm{E}-05$ & $3.7 \mathrm{E}-05$ \\
\hline $\mathrm{Eu}-152 \mathrm{~m}$ & $6.9 \mathrm{E}-06$ & $5.4 \mathrm{E}-06$ & Fr-221 & $2.6 \mathrm{E}-09$ & $2.3 \mathrm{E}-09$ \\
\hline Eu-154 & $1.5 \mathrm{E}-01$ & $1.2 \mathrm{E}-01$ & Fr-223 & $1.8 \mathrm{E}-06$ & $1.6 \mathrm{E}-06$ \\
\hline Eu-155 & $6.3 \mathrm{E}-03$ & $4.8 \mathrm{E}-03$ & $\mathrm{Ra}-223$ & $1.8 \mathrm{E}-01$ & $1.4 \mathrm{E}-01$ \\
\hline Eu-156 & $1.9 \mathrm{E}-03$ & $1.5 \mathrm{E}-03$ & $\mathrm{Ra}-224$ & $6.5 \mathrm{E}-02$ & $4.9 \mathrm{E}-02$ \\
\hline $\mathrm{Gd}-152^{\mathrm{b}}$ & (Pu-239) & (Pu-239) & $\mathrm{Ra}-225$ & $1.2 \mathrm{E}-01$ & 8.7E-02 \\
\hline $\mathrm{Tb}-160$ & $5.0 \mathrm{E}-03$ & $3.9 \mathrm{E}-03$ & $\mathrm{Ra}-226$ & $3.6 \mathrm{E}-01$ & 2.7E-01 \\
\hline Ho-166 & $6.6 \mathrm{E}-05$ & $4.9 \mathrm{E}-05$ & $\mathrm{Ra}-228$ & $1.5 \mathrm{E}-01$ & $1.1 \mathrm{E}-01$ \\
\hline Ho- $166 \mathrm{~m}$ & $6.2 \mathrm{E}-01$ & $4.8 \mathrm{E}-01$ & Ac-225 & $1.1 \mathrm{E}-01$ & $8.0 \mathrm{E}-02$ \\
\hline Hf-181 & $1.9 \mathrm{E}-03$ & $1.5 \mathrm{E}-03$ & Ac-227 & $1.1 \mathrm{E}+01$ & $8.4 \mathrm{E}+00$ \\
\hline W-181 & $3.6 \mathrm{E}-04$ & $2.7 \mathrm{E}-04$ & Ac-228 & $9.8 \mathrm{E}-04$ & $7.5 \mathrm{E}-04$ \\
\hline W-185 & $4.7 \mathrm{E}-04$ & $3.6 \mathrm{E}-04$ & Th-227 & $2.0 \mathrm{E}-01$ & $1.5 \mathrm{E}-01$ \\
\hline W-187 & $3.8 \mathrm{E}-05$ & $2.9 \mathrm{E}-05$ & Th-228 & $4.3 E+\infty 0$ & $3.2 \mathrm{E}+00$ \\
\hline $\operatorname{Re}-187$ & $1.4 \mathrm{E}-05$ & $1.0 \mathrm{E}-05$ & Th-229 & $1.2 \mathrm{E}+01$ & $8.9 E+00$ \\
\hline Ir-192 & $4.3 \mathrm{E}-03$ & $3.3 \mathrm{E}-03$ & Th-230 & $4.3 \mathrm{E}+00$ & $3.2 \mathrm{E}+00$ \\
\hline $\mathrm{Hg}-203$ & $2.7 \mathrm{E}-03$ & $2.1 \mathrm{E}-03$ & Th-231 & $1.8 \mathrm{E}-05$ & $1.3 \mathrm{E}-05$ \\
\hline Tl-207 & 5.3E-12 & $4.6 \mathrm{E}-12$ & Th-232 & $6.2 \mathrm{E}+00$ & $4.5 \mathrm{E}+\infty 0$ \\
\hline T1-208 & $8.5 \mathrm{E}-12$ & $7.4 \mathrm{E}-12$ & Th-234 & $1.9 \mathrm{E}-03$ & $1.4 \mathrm{E}-03$ \\
\hline T1-209 & $2.2 \mathrm{E}-14$ & $1.9 \mathrm{E}-14$ & $\mathrm{~Pa}-231$ & $8.9 E+00$ & $6.6 \mathrm{E}+00$ \\
\hline $\mathrm{Pb}-209$ & $8.4 \mathrm{E}-07$ & $6.6 \mathrm{E}-07$ & $\mathrm{~Pa}-233$ & $8.1 \mathrm{E}-04$ & $6.2 \mathrm{E}-04$ \\
\hline $\mathrm{Pb}-210$ & $1.1 \mathrm{E}+00$ & $8.4 \mathrm{E}-01$ & $\mathrm{~Pa}-234$ & $4.5 \mathrm{E}-05$ & $3.5 \mathrm{E}-05$ \\
\hline $\mathrm{Pb}-211$ & $1.5 \mathrm{E}-05$ & $1.3 \mathrm{E}-05$ & $\mathrm{~Pa}-234 \mathrm{~m}$ & $7.8 \mathrm{E}-24$ & $6.8 \mathrm{E}-24$ \\
\hline $\mathrm{Pb}-212$ & $2.3 \mathrm{E}-03$ & $1.7 \mathrm{E}-03$ & $\mathrm{U}-232$ & $8.6 E+00$ & $6.3 \mathrm{E}+00$ \\
\hline $\mathrm{Pb}-214$ & $1.1 \mathrm{E}-06$ & $9.6 \mathrm{E}-07$ & U-233 & $2.4 \mathrm{E}+00$ & $1.8 \mathrm{E}+00$ \\
\hline $\mathrm{Bi}-210$ & $3.4 \mathrm{E}-03$ & $2.5 \mathrm{E}-03$ & U-234 & $2.4 \mathrm{E}+00$ & $1.8 \mathrm{E}+00$ \\
\hline $\mathrm{Bi}-211$ & $1.0 \mathrm{E}-14$ & $8.8 \mathrm{E}-15$ & U-235 & $2.3 \mathrm{E}+00$ & $1.7 \mathrm{E}+\infty 0$ \\
\hline $\mathrm{Bi}-212$ & $1.0 \mathrm{E}-04$ & $9.0 \mathrm{E}-05$ & U-236 & $2.3 E+00$ & $1.7 \mathrm{E}+00$ \\
\hline $\mathrm{Bi}-213$ & $2.9 \mathrm{E}-06$ & $2.6 \mathrm{E}-06$ & U-237 & $2.4 \mathrm{E}-04$ & $1.8 \mathrm{E}-04$ \\
\hline Bi-214 & 7.5E-07 & $6.6 \mathrm{E}-07$ & U-238 & $2.1 E+\infty 0$ & $1.6 \mathrm{E}+\infty 0$ \\
\hline Po-210 & 4.1E-01 & $3.1 \mathrm{E}-01$ & $\mathrm{U}-240$ & $3.6 \mathrm{E}-05$ & $2.7 \mathrm{E}-05$ \\
\hline
\end{tabular}


HNF-3602, Vol. 1

Table 4-10. 200 West Area--CAP88-PC Unit Dose Factors by Effective Release Height $^{\mathrm{a}}$ (mrem/Ci).

\begin{tabular}{|c|c|c|c|c|c|}
\hline \multirow{2}{*}{ Nuclide } & \multicolumn{2}{|c|}{$\begin{array}{c}\text { Effective } \\
\text { Release Height }\end{array}$} & \multirow{2}{*}{ Nuclide } & \multicolumn{2}{|c|}{$\begin{array}{c}\text { Effective } \\
\text { Release Height }\end{array}$} \\
\hline & $\mathrm{H}_{\mathrm{eff}}<40 \mathrm{~m}$ & $\mathrm{H}_{\text {eff } 2} 40 \mathrm{~m}$ & & $\mathrm{H}_{\mathrm{eff}}<40 \mathrm{~m}$ & $\mathrm{H}_{\text {eff } z} 40 \mathrm{~m}$ \\
\hline $\begin{array}{c}\text { Np-237 } \\
\text { Np-238 } \\
\text { Np-239 } \\
\text { Np-240 } \\
\text { Np-240m } \\
\text { Pu-236 } \\
\text { Pu-238 } \\
\text { Pu-239 } \\
\text { Pu-240 } \\
\text { Pu-241 }\end{array}$ & $\begin{array}{c}8.9 \mathrm{E}+00 \\
6.0 \mathrm{E}-04 \\
9.1 \mathrm{E}-05 \\
3.2 \mathrm{E}-06 \\
2.9 \mathrm{E}-09 \\
1.6 \mathrm{E}+00 \\
5.9 \mathrm{E}+00 \\
6.4 \mathrm{E}+00 \\
6.4 \mathrm{E}+00 \\
1.0 \mathrm{E}-01 \\
\end{array}$ & $\begin{array}{c}6.6 \mathrm{E}+00 \\
4.5 \mathrm{E}-04 \\
6.9 \mathrm{E}-05 \\
2.8 \mathrm{E}-06 \\
2.5 \mathrm{E}-09 \\
1.2 \mathrm{E}+00 \\
4.4 \mathrm{E}+00 \\
4.7 \mathrm{E}+00 \\
4.7 \mathrm{E}+00 \\
7.4 \mathrm{E}-02\end{array}$ &  & & \\
\hline $\begin{array}{c}\text { Pu-242 } \\
\text { Pu-243 } \\
\text { Pu-244 } \\
\text { Am-241 } \\
\text { Am-242 } \\
\text { Am-242m } \\
\text { Am-243 } \\
\text { Cm-242 } \\
\text { Cm-243 } \\
\text { Cm-244 }\end{array}$ & $\begin{array}{c}6.1 \mathrm{E}+00 \\
2.7 \mathrm{E}-06 \\
6.0 \mathrm{E}+00 \\
9.8 \mathrm{E}+00 \\
8.6 \mathrm{E}-04 \\
9.4 \mathrm{E}+00 \\
9.8 \mathrm{E}+00 \\
3.2 \mathrm{E}-01 \\
6.6 \mathrm{E}+00 \\
5.2 \mathrm{E}+\infty \\
\end{array}$ & $\begin{array}{c}4.5 \mathrm{E}+00 \\
2.1 \mathrm{E}-06 \\
4.5 \mathrm{E}+\infty \\
7.2 \mathrm{E}+\infty 0 \\
6.5 \mathrm{E}-04 \\
7.0 \mathrm{E}+\infty \\
7.3 \mathrm{E}+00 \\
2.4 \mathrm{E}-01 \\
4.9 \mathrm{E}+00 \\
3.8 \mathrm{E}+00 \\
\end{array}$ & & & \\
\hline $\begin{array}{c}\mathrm{Cm}-245 \\
\mathrm{Cm}-246 \\
\mathrm{Cm}-247 \\
\mathrm{Cm}-248 \\
\mathrm{Cf}-252 \\
\end{array}$ & $\begin{array}{l}1.0 \mathrm{E}+01 \\
1.0 \mathrm{E}+01 \\
9.3 \mathrm{E}+00 \\
3.7 \mathrm{E}+01 \\
2.8 \mathrm{E}+00\end{array}$ & $\begin{array}{l}7.5 \mathrm{E}+00 \\
7.4 \mathrm{E}+00 \\
6.9 \mathrm{E}+\infty 0 \\
2.7 \mathrm{E}+01 \\
2.0 \mathrm{E}+\infty 0\end{array}$ & & & \\
\hline
\end{tabular}

2 The effective release height, $x$, is in meters.

b CAP88-PC Ver. 1.0 does not calculate doses for Be-10, Se-79, Cd-113, Cd-113m, and Gd-152. Recommend substituting the unit dose factors of the nuclides in parentheses.

c Extremely short lived nuclide, resulting in an offsite dose of zero.

d Short lived radon nuclides only provide the mechanism to circumvent the emission control device. The unit dose factors for the recommended progeny should be used instead.

However, 1 ci of Rn does not equal 1 ci of Pb. Multiply the activity of Rn-219 by 0.0018 to get the activity of $\mathrm{Pb}-211$. Multiply the activity of $\mathrm{Rn}-220$ by 0.0014 to get the activity of $\mathrm{Pb}-212$. 
Table 4-11. 300 East Area--CAP88-PC Unit Dose Factors by Effective Release Height $^{\mathrm{a}}$ (mrem/Ci).

\begin{tabular}{|c|c|c|c|c|c|}
\hline \multirow{2}{*}{ Nuclide } & \multicolumn{2}{|c|}{$\begin{array}{c}\text { Effective } \\
\text { Release Height }\end{array}$} & \multirow{2}{*}{ Nuclide } & \multicolumn{2}{|c|}{$\begin{array}{c}\text { Effective } \\
\text { Release Height }\end{array}$} \\
\hline & $\mathrm{H}_{\mathrm{eff}}<40 \mathrm{~m}$ & $\mathrm{H}_{\text {eff }} \geq 40 \mathrm{~m}$ & & $\mathrm{H}_{\mathrm{eff}}<40 \mathrm{~m}$ & $\mathrm{H}_{\text {eff }} \geq 40 \mathrm{~m}$ \\
\hline H-3 & $4.8 \mathrm{E}-04$ & $1.4 \mathrm{E}-04$ & $\mathrm{Kr}-85$ & $1.1 \mathrm{E}-06$ & $3.1 \mathrm{E}-07$ \\
\hline $\mathrm{Be}-7$ & $4.5 \mathrm{E}-03$ & $1.5 \mathrm{E}-03$ & $\mathrm{Kr}-85 \mathrm{~m}$ & $5.0 \mathrm{E}-05$ & $1.5 \mathrm{E}-05$ \\
\hline $\mathrm{Be}-10^{\mathrm{b}}$ & $(\mathrm{Sr}-90)$ & $(\mathrm{Sr}-90)$ & $\mathrm{Kr}-87$ & $2.6 \mathrm{E}-04$ & $7.8 \mathrm{E}-05$ \\
\hline C-11 & $2.8 \mathrm{E}-04$ & $8.8 \mathrm{E}-05$ & $\mathrm{Kr}-88$ & $6.9 \mathrm{E}-04$ & $2.1 \mathrm{E}-04$ \\
\hline C-14 & $3.7 \mathrm{E}-02$ & $1.1 \mathrm{E}-02$ & $\mathrm{Kr}-89$ & $2.4 \mathrm{E}-04$ & $8.1 \mathrm{E}-05$ \\
\hline $\mathrm{C}-15^{\mathrm{c}}$ & 0.0 & 0.0 & $\mathrm{Kr}-90$ & $6.5 \mathrm{E}-06$ & $2.4 \mathrm{E}-06$ \\
\hline $\mathrm{N}-13$ & $2.2 \mathrm{E}-04$ & 7.1E-05 & $\mathrm{Rb}-86$ & $6.7 \mathrm{E}-02$ & $2.2 \mathrm{E}-02$ \\
\hline $0-15$ & $8.1 \mathrm{E}-05$ & $2.9 \mathrm{E}-05$ & $\mathrm{Rb}-87$ & $2.2 \mathrm{E}-01$ & $7.1 \mathrm{E}-02$ \\
\hline F-18 & 4.4E-04 & $1.4 \mathrm{E}-04$ & $\mathrm{Rb}-88$ & $2.2 \mathrm{E}-04$ & $7.1 \mathrm{E}-05$ \\
\hline $\mathrm{Na}-22$ & $3.0 \mathrm{E}+\infty 0$ & $9.7 \mathrm{E}-01$ & $\mathrm{Rb}-89$ & $5.6 \mathrm{E}-04$ & $1.8 \mathrm{E}-04$ \\
\hline $\mathrm{Na}-24$ & $5.5 \mathrm{E}-03$ & $1.7 \mathrm{E}-03$ & Rb-90 & $2.5 \mathrm{E}-04$ & $9.0 \mathrm{E}-05$ \\
\hline P-32 & $6.7 \mathrm{E}-02$ & $2.2 \mathrm{E}-02$ & $\mathrm{Rb}-90 \mathrm{~m}$ & $5.2 \mathrm{E}-04$ & $1.8 \mathrm{E}-04$ \\
\hline S-35 & $9.5 \mathrm{E}-03$ & $3.1 \mathrm{E}-03$ & Sr-89 & $4.0 \mathrm{E}-02$ & $1.3 \mathrm{E}-02$ \\
\hline $\mathrm{Ar}-41$ & $4.0 \mathrm{E}-04$ & $1.2 \mathrm{E}-04$ & Sr-90 & $2.6 \mathrm{E}+00$ & $8.5 \mathrm{E}-01$ \\
\hline $\mathrm{K}-40$ & $2.9 \mathrm{E}+00$ & $9.3 E-01$ & $\mathrm{Sr}-91$ & $1.2 \mathrm{E}-03$ & $3.6 \mathrm{E}-04$ \\
\hline $\mathrm{Ca}-41$ & $2.4 \mathrm{E}-04$ & 7.7E-05 & $\mathrm{Sr}-92$ & $9.4 \mathrm{E}-04$ & $2.9 \mathrm{E}-04$ \\
\hline $\mathrm{Sc}-46$ & $2.8 \mathrm{E}-01$ & $9.1 \mathrm{E}-02$ & Y-90 & $8.3 \mathrm{E}-03$ & $2.6 \mathrm{E}-03$ \\
\hline $\mathrm{Cr}-51$ & $2.0 \mathrm{E}-03$ & $6.6 \mathrm{E}-04$ & $Y-90 m$ & $3.1 \mathrm{E}-04$ & $9.7 \mathrm{E}-05$ \\
\hline $\mathrm{Mn}-54$ & $3.8 \mathrm{E}-01$ & $1.2 \mathrm{E}-01$ & Y-91 & $6.0 \mathrm{E}-02$ & $1.9 \mathrm{E}-02$ \\
\hline $\mathrm{Mn}-56$ & $1.1 \mathrm{E}-03$ & $3.3 \mathrm{E}-04$ & $\mathrm{Y}-91 \mathrm{~m}$ & $1.9 \mathrm{E}-04$ & $5.9 \mathrm{E}-05$ \\
\hline $\mathrm{Fe}-55$ & $5.4 \mathrm{E}-03$ & $1.7 \mathrm{E}-03$ & Y-92 & $7.5 \mathrm{E}-04$ & $2.3 \mathrm{E}-04$ \\
\hline $\mathrm{Fe}-59$ & $1.1 \mathrm{E}-01$ & $3.5 \mathrm{E}-02$ & $Y-93$ & $1.6 \mathrm{E}-03$ & 4.7E-04 \\
\hline $\mathrm{Co}-57$ & $6.6 \mathrm{E}-02$ & 2.1E-02 & Zr-93 & $3.1 \mathrm{E}-02$ & $9.4 \mathrm{E}-03$ \\
\hline $\mathrm{Co}-58$ & $1.3 \mathrm{E}-01$ & $4.1 \mathrm{E}-02$ & Zr-95 & $9.1 \mathrm{E}-02$ & $2.9 \mathrm{E}-02$ \\
\hline $\mathrm{Co}-60$ & $5.7 \mathrm{E}+00$ & $1.9 \mathrm{E}+\infty 0$ & $\mathrm{Nb}-93 \mathrm{~m}$ & $4.9 \mathrm{E}-02$ & $1.5 \mathrm{E}-02$ \\
\hline $\mathrm{Ni}-59$ & $7.1 \mathrm{E}-03$ & $2.3 \mathrm{E}-03$ & $\mathrm{Nb}-94$ & $1.9 \mathrm{E}+01$ & $6.1 E+\infty 0$ \\
\hline $\mathrm{Ni}-63$ & $6.1 \mathrm{E}-03$ & $2.0 \mathrm{E}-03$ & $\mathrm{Nb}-95$ & $1.1 \mathrm{E}-01$ & $3.6 \mathrm{E}-02$ \\
\hline $\mathrm{Ni}-65$ & $4.2 \mathrm{E}-04$ & $1.3 \mathrm{E}-04$ & $\mathrm{Nb}-95 \mathrm{~m}$ & 7.1E-03 & $2.3 \mathrm{E}-03$ \\
\hline $\mathrm{Cu}-64$ & $3.9 \mathrm{E}-04$ & $1.2 \mathrm{E}-04$ & $\mathrm{Nb}-97$ & $3.1 \mathrm{E}-04$ & $9.6 \mathrm{E}-05$ \\
\hline $\mathrm{Zn}-65$ & $5.7 \mathrm{E}-01$ & $1.9 \mathrm{E}-01$ & $\mathrm{Nb}-97 \mathrm{~m}$ & 2.1E-05 & $7.6 \mathrm{E}-06$ \\
\hline $\mathrm{Zn}-69 \mathrm{~m}$ & $1.1 \mathrm{E}-03$ & $3.3 \mathrm{E}-04$ & Mo-93 & $6.5 \mathrm{E}-02$ & $2.1 \mathrm{E}-02$ \\
\hline $\mathrm{Zn}-69$ & $3.3 \mathrm{E}-05$ & $1.0 \mathrm{E}-05$ & Mo-99 & $4.2 \mathrm{E}-03$ & $1.3 \mathrm{E}-03$ \\
\hline $\mathrm{Ga}-67$ & $1.5 \mathrm{E}-03$ & 4.6E-04 & Tc-97 & $1.3 \mathrm{E}-01$ & $4.3 \mathrm{E}-02$ \\
\hline As-76 & $3.4 \mathrm{E}-03$ & $1.0 \mathrm{E}-03$ & Tc-99 & $5.2 \mathrm{E}-01$ & $1.7 \mathrm{E}-01$ \\
\hline $\mathrm{Se}-79^{\mathrm{b}}$ & (Pu-241) & (Pu-241) & Tc-99m & $1.1 \mathrm{E}-04$ & $3.4 \mathrm{E}-05$ \\
\hline $\mathrm{Br}-82$ & $7.7 \mathrm{E}-03$ & $2.5 \mathrm{E}-03$ & Tc-101 & $8.0 \mathrm{E}-05$ & $2.6 \mathrm{E}-05$ \\
\hline $\mathrm{Br}-83$ & $3.2 \mathrm{E}-06$ & $1.0 \mathrm{E}-06$ & Ru-97 & $1.6 \mathrm{E}-03$ & $5.0 \mathrm{E}-04$ \\
\hline $\mathrm{Br}-84$ & $5.8 \mathrm{E}-04$ & $1.8 \mathrm{E}-04$ & Ru-103 & $4.3 \mathrm{E}-02$ & $1.4 \mathrm{E}-02$ \\
\hline $\mathrm{Br}-85$ & 7.4E-06 & $2.6 \mathrm{E}-06$ & $\mathrm{Ru}-105$ & $7.8 \mathrm{E}-04$ & $2.4 \mathrm{E}-04$ \\
\hline $\mathrm{Kr}-83 \mathrm{~m}$ & $7.1 \mathrm{E}-08$ & $2.1 \mathrm{E}-08$ & Ru-106 & $3.7 \mathrm{E}-01$ & $1.1 \mathrm{E}-01$ \\
\hline
\end{tabular}


HNF-3602, Vol. 1

Table 4-11. 300 East Area--CAP88-PC Unit Dose Factors by Effective Release

Height $^{\mathrm{a}}(\mathrm{mrem} / \mathrm{Ci})$

\begin{tabular}{|c|c|c|c|c|c|}
\hline \multirow{2}{*}{ Nuclide } & \multicolumn{2}{|c|}{$\begin{array}{c}\text { Effective } \\
\text { Release Height }\end{array}$} & \multirow{2}{*}{ Nuclide } & \multicolumn{2}{|c|}{$\begin{array}{c}\text { Effective } \\
\text { Release Height }\end{array}$} \\
\hline & $\mathrm{H}_{\mathrm{eff}}<40 \mathrm{~m}$ & $\mathrm{H}_{\text {eff }} \geq 40 \mathrm{~m}$ & & $\mathrm{H}_{\mathrm{eff}}<40 \mathrm{~m}$ & $\mathrm{H}_{\text {eff }} \geq 40 \mathrm{~m}$ \\
\hline $\mathrm{Rh}-103 \mathrm{~m}$ & $4.2 \mathrm{E}-06$ & $1.3 \mathrm{E}-06$ & I-129 & $5.6 \mathrm{E}+01$ & $2.4 \mathrm{E}+01$ \\
\hline Rh-105 & $1.2 \mathrm{E}-03$ & 3.7E-04 & $\mathrm{I}-130$ & $2.2 \mathrm{E}-02$ & $9.6 \mathrm{E}-03$ \\
\hline $\mathrm{Rh}-105 \mathrm{~m}$ & $4.1 \mathrm{E}-07$ & $1.5 \mathrm{E}-07$ & I-131 & $1.8 \mathrm{E}+\infty$ & $8.0 \mathrm{E}-01$ \\
\hline Rh-106 & $7.4 \mathrm{E}-07$ & $2.8 \mathrm{E}-07$ & I-132 & 4.3E-03 & $1.9 \mathrm{E}-03$ \\
\hline $\mathrm{Pd}-107$ & $8.9 \mathrm{E}-03$ & $2.7 \mathrm{E}-03$ & I-133 & $2.2 \mathrm{E}-02$ & $9.6 \mathrm{E}-03$ \\
\hline Pd-109 & $9.2 \mathrm{E}-04$ & $2.8 \mathrm{E}-04$ & I-134 & $2.1 \mathrm{E}-03$ & $9.1 \mathrm{E}-04$ \\
\hline $\mathrm{Ag}-109 \mathrm{~m}$ & $4.5 \mathrm{E}-08$ & $1.7 \mathrm{E}-08$ & I-135 & $7.8 \mathrm{E}-03$ & $3.4 \mathrm{E}-03$ \\
\hline Ag-110 & $4.8 \mathrm{E}-08$ & $1.9 \mathrm{E}-08$ & $\mathrm{Xe}-122$ & $2.1 \mathrm{E}-05$ & $6.2 \mathrm{E}-06$ \\
\hline $\mathrm{Ag}-110 \mathrm{~m}$ & $1.1 \mathrm{E}+00$ & $3.6 \mathrm{E}-01$ & $\mathrm{Xe}-123$ & $1.9 \mathrm{E}-04$ & 5.7E-05 \\
\hline Ag-111 & $2.4 \mathrm{E}-02$ & $7.6 \mathrm{E}-03$ & $\mathrm{Xe}-125$ & $7.8 \mathrm{E}-05$ & 2.3E-05 \\
\hline $\mathrm{Cd}-113^{\mathrm{b}}$ & (Pu-241) & (Pu-241) & $\mathrm{Xe}-127$ & $8.2 \mathrm{E}-05$ & $2.4 \mathrm{E}-05$ \\
\hline $\mathrm{Cd}-113 \mathrm{~m}^{\mathrm{b}}$ & (Pu-241) & (Pu-241) & $\mathrm{Xe}-131 \mathrm{~m}$ & $3.0 \mathrm{E}-06$ & $8.8 \mathrm{E}-07$ \\
\hline $\mathrm{Cd}-115$ & $4.6 \mathrm{E}-03$ & $1.5 \mathrm{E}-03$ & $\mathrm{Xe}-133$ & 1.1E-05 & $3.2 \mathrm{E}-06$ \\
\hline $\mathrm{Cd}-115 \mathrm{~m}$ & $8.3 \mathrm{E}-02$ & $2.7 \mathrm{E}-02$ & $\mathrm{Xe}-133 \mathrm{~m}$ & $9.6 \mathrm{E}-06$ & $2.8 \mathrm{E}-06$ \\
\hline $\mathrm{In}-113 \mathrm{~m}$ & $1.3 \mathrm{E}-04$ & $3.9 \mathrm{E}-05$ & $\mathrm{Xe}-135$ & 7.7E-05 & $2.3 \mathrm{E}-05$ \\
\hline In-115 & $1.4 \mathrm{E}+00$ & $4.5 \mathrm{E}-01$ & $\mathrm{Xe}-135 \mathrm{~m}$ & $1.0 \mathrm{E}-04$ & $3.1 \mathrm{E}-05$ \\
\hline In-115m & $1.8 \mathrm{E}-04$ & $5.6 \mathrm{E}-05$ & $\mathrm{Xe}-137$ & $2.6 \mathrm{E}-05$ & $8.7 \mathrm{E}-06$ \\
\hline Sn-113 & $5.1 \mathrm{E}-02$ & $1.7 \mathrm{E}-02$ & $\mathrm{Xe}-138$ & $2.9 \mathrm{E}-04$ & $9.0 \mathrm{E}-05$ \\
\hline $\mathrm{Sn}-123$ & $1.2 \mathrm{E}-03$ & $3.9 \mathrm{E}-04$ & Cs-134 & $2.3 E+00$ & $7.5 \mathrm{E}-01$ \\
\hline Sn-125 & $5.5 \mathrm{E}-02$ & $1.8 \mathrm{E}-02$ & $\mathrm{Cs}-134 \mathrm{~m}$ & $3.4 \mathrm{E}-05$ & $1.1 \mathrm{E}-05$ \\
\hline Sn-126 & $1.1 \mathrm{E}+00$ & $3.5 \mathrm{E}-01$ & Cs-135 & $1.0 \mathrm{E}-01$ & $3.3 \mathrm{E}-02$ \\
\hline Sb-124 & $2.0 \mathrm{E}-01$ & $6.5 \mathrm{E}-02$ & Cs-136 & $8.6 \mathrm{E}-02$ & $2.8 \mathrm{E}-02$ \\
\hline $\mathrm{Sb}-125$ & $6.1 \mathrm{E}-01$ & $2.0 \mathrm{E}-01$ & Cs-137 & $6.2 \mathrm{E}-01$ & $2.0 \mathrm{E}-01$ \\
\hline $\mathrm{Sb}-126$ & $7.6 \mathrm{E}-02$ & $2.5 \mathrm{E}-02$ & Cs-138 & $7.9 \mathrm{E}-04$ & $2.5 \mathrm{E}-04$ \\
\hline$S b-126 b$ & $4.3 \mathrm{E}-04$ & $1.4 \mathrm{E}-04$ & Cs-139 & $6.7 \mathrm{E}-05$ & $2.2 \mathrm{E}-05$ \\
\hline $\mathrm{Sb}-127$ & $1.1 \mathrm{E}-02$ & $3.5 \mathrm{E}-03$ & $\mathrm{Ba}-133$ & $1.7 E+\infty 0$ & $5.6 \mathrm{E}-01$ \\
\hline $\mathrm{Te}-125 \mathrm{~m}$ & $2.4 \mathrm{E}-02$ & 7.7E-03 & $\mathrm{Ba}-133 \mathrm{~m}$ & $7.6 \mathrm{E}-04$ & $2.4 \mathrm{E}-04$ \\
\hline $\mathrm{Te}-127$ & $2.3 \mathrm{E}-04$ & $6.9 \mathrm{E}-05$ & $\mathrm{Ba}-137 \mathrm{~m}$ & $5.9 \mathrm{E}-05$ & $2.1 \mathrm{E}-05$ \\
\hline $\mathrm{Te}-127 \mathrm{~m}$ & $5.9 \mathrm{E}-02$ & $1.9 \mathrm{E}-02$ & $\mathrm{Ba}-139$ & $1.1 \mathrm{E}-04$ & 3.3E-05 \\
\hline Te-129 & $8.6 \mathrm{E}-05$ & $2.6 \mathrm{E}-05$ & $\mathrm{Ba}-140$ & $2.5 \mathrm{E}-02$ & $7.9 \mathrm{E}-03$ \\
\hline $\mathrm{Te}-129 \mathrm{~m}$ & $5.8 \mathrm{E}-02$ & $1.9 \mathrm{E}-02$ & $\mathrm{Ba}-141$ & $2.3 \mathrm{E}-04$ & $7.3 \mathrm{E}-05$ \\
\hline $\mathrm{Te}-131$ & $1.7 \mathrm{E}-04$ & $5.3 \mathrm{E}-05$ & $\mathrm{Ba}-142$ & $2.0 \mathrm{E}-04$ & $6.4 \mathrm{E}-05$ \\
\hline Te-131m & $5.8 \mathrm{E}-03$ & $1.8 \mathrm{E}-03$ & La-140 & $9.7 \mathrm{E}-03$ & $3.1 \mathrm{E}-03$ \\
\hline $\mathrm{Te}-132$ & $9.8 \mathrm{E}-03$ & $3.1 \mathrm{E}-03$ & La-141 & $2.2 \mathrm{E}-05$ & $6.8 \mathrm{E}-06$ \\
\hline Te-133 & $2.1 \mathrm{E}-04$ & $6.9 \mathrm{E}-05$ & La-142 & $1.1 \mathrm{E}-03$ & $3.5 \mathrm{E}-04$ \\
\hline $\mathrm{Te}-133 \mathrm{~m}$ & $7.6 \mathrm{E}-04$ & $2.4 \mathrm{E}-04$ & Ce-141 & $1.8 \mathrm{E}-02$ & $5.6 \mathrm{E}-03$ \\
\hline $\mathrm{Te}-134$ & 2.7E-04 & $8.5 \mathrm{E}-05$ & Ce-143 & $2.9 \mathrm{E}-03$ & $9.0 \mathrm{E}-04$ \\
\hline I-122 & $1.0 \mathrm{E}-04$ & $5.0 \mathrm{E}-05$ & Ce-144 & $2.9 \mathrm{E}-01$ & $9.0 \mathrm{E}-02$ \\
\hline I- 123 & $2.0 \mathrm{E}-03$ & $8.7 \mathrm{E}-04$ & Pr-143 & $1.4 \mathrm{E}-02$ & $4.4 \mathrm{E}-03$ \\
\hline I-125 & $3.3 E+00$ & $1.5 E+\infty$ & $\mathrm{Pr}-144$ & 4.2E-05 & $1.3 \mathrm{E}-05$ \\
\hline
\end{tabular}


HNF-3602, Vol. 1

Table 4-11. 300 East Area--CAP88-PC Unit Dose Factors by Effective Release Height $^{\mathrm{a}}(\mathrm{mrem} / \mathrm{Ci})$.

\begin{tabular}{|c|c|c|c|c|c|}
\hline \multirow{2}{*}{ Nuclide } & \multicolumn{2}{|c|}{$\begin{array}{c}\text { Effective } \\
\text { Release Height }\end{array}$} & \multirow{2}{*}{ Nuclide } & \multicolumn{2}{|c|}{$\begin{array}{c}\text { Effective } \\
\text { Release Height }\end{array}$} \\
\hline & $\mathrm{H}_{\mathrm{eff}}<40 \mathrm{~m}$ & $\mathrm{H}_{\text {eff }} \geq 40 \mathrm{~m}$ & & $\mathrm{H}_{\mathrm{eff}}<40 \mathrm{~m}$ & $\mathrm{H}_{\text {eff }} \geq 40 \mathrm{~m}$ \\
\hline Pr- $144 m$ & $1.2 \mathrm{E}-05$ & $3.9 \mathrm{E}-06$ & Po-211 ${ }^{\mathrm{c}}$ & 0.0 & 0.0 \\
\hline $\mathrm{Nd}-147$ & $1.4 \mathrm{E}-02$ & $4.3 \mathrm{E}-03$ & Po-212 & 0.0 & 0.0 \\
\hline Pm-147 & 2.6E-02 & $7.8 \mathrm{E}-03$ & $\mathrm{P}_{0}-213^{\mathrm{c}}$ & 0.0 & 0.0 \\
\hline Pm-148 & $2.0 \mathrm{E}-02$ & $6.4 \mathrm{E}-03$ & Po-214 & 0.0 & 0.0 \\
\hline $\mathrm{Pm}-148 \mathrm{~m}$ & $1.8 \mathrm{E}-01$ & $5.8 \mathrm{E}-02$ & Po-215 & 0.0 & 0.0 \\
\hline Pm-149 & $2.6 \mathrm{E}-03$ & $8.1 \mathrm{E}-04$ & Po-216 $6^{\mathrm{C}}$ & 0.0 & 0.0 \\
\hline Pm-151 & $7.0 \mathrm{E}-04$ & $2.3 \mathrm{E}-04$ & Po-218 & $1.0 \mathrm{E}-05$ & $3.6 \mathrm{E}-06$ \\
\hline $\mathrm{Sm}-147$ & $4.0 \mathrm{E}+01$ & $1.2 \mathrm{E}+01$ & At $-217^{c}$ & 0.0 & 0.0 \\
\hline $\mathrm{Sm}-151$ & $1.8 \mathrm{E}-02$ & $5.4 \mathrm{E}-03$ & Rn-219 & $(\mathrm{Pb}-211)$ & $(\mathrm{Pb}-211)$ \\
\hline $\mathrm{Sm}-153$ & $1.9 \mathrm{E}-03$ & $5.8 \mathrm{E}-04$ & $\mathrm{Rn}-220^{\mathrm{d}}$ & $(\mathrm{Pb}-212)$ & $(\mathrm{Pb}-212)$ \\
\hline Eu-152 & $5.6 \mathrm{E}+00$ & $1.8 \mathrm{E}+00$ & $\mathrm{Rn}-222$ & $1.4 \mathrm{E}-03$ & $4.0 \mathrm{E}-04$ \\
\hline $\mathrm{Eu}-152 \mathrm{~m}$ & $2.7 \mathrm{E}-04$ & $8.6 \mathrm{E}-05$ & Fr-221 & $1.1 \mathrm{E}-03$. & $3.8 \mathrm{E}-04$ \\
\hline $\mathrm{Eu}-154$ & $4.6 \mathrm{E}+00$ & $1.5 \mathrm{E}+00$ & Fr-223 & 1.2E-03 & 3.7E-04 \\
\hline Eu-155 & $1.9 \mathrm{E}-01$ & $6.0 \mathrm{E}-02$ & $\mathrm{Ra}-223$ & $5.7 \mathrm{E}+\infty$ & $1.7 \mathrm{E}+00$ \\
\hline Eu-156 & 5.7E-02 & $1.8 \mathrm{E}-02$ & $\mathrm{Ra}-224$ & $2.1 E+\infty 0$ & $6.3 \mathrm{E}-01$ \\
\hline $\mathrm{Gd}-152^{\mathrm{b}}$ & (Pu-239) & (Pu-239) & $\mathrm{Ra}-225$ & $3.5 \mathrm{E}+\infty 0$ & $1.1 E+00$ \\
\hline $\mathrm{Tb}-160$ & $1.5 \mathrm{E}-01$ & $4.8 \mathrm{E}-02$ & $\mathrm{Ra}-226$ & $1.1 \mathrm{E}+01$ & $3.4 \mathrm{E}+00$ \\
\hline Ho-166 & $2.2 \mathrm{E}-03$ & $6.8 \mathrm{E}-04$ & $\mathrm{Ra}-228$ & $4.4 \mathrm{E}+00$ & $1.4 \mathrm{E}+00$ \\
\hline $\mathrm{Ho}-166 \mathrm{~m}$ & $1.8 \mathrm{E}+01$ & $6.0 \mathrm{E}+00$ & Ac-225 & $3.4 \mathrm{E}+00$ & $1.0 \mathrm{E}+\infty 0$ \\
\hline Hf-181 & $5.7 \mathrm{E}-02$ & $1.8 \mathrm{E}-02$ & Ac-227 & $3.5 E+02$ & $1.1 \mathrm{E}+02$ \\
\hline W-181 & $1.1 \mathrm{E}-02$ & $3.4 \mathrm{E}-03$ & Ac-228 & $4.5 \mathrm{E}-02$ & $1.4 \mathrm{E}-02$ \\
\hline W-185 & $1.4 \mathrm{E}-02$ & $4.5 \mathrm{E}-03$ & Th-227 & $6.3 E+00$ & $1.9 E+00$ \\
\hline W-187 & $1.3 \mathrm{E}-03$ & $4.0 \mathrm{E}-04$ & Th-228 & $1.3 \mathrm{E}+02$ & $4.0 \mathrm{E}+01$ \\
\hline $\operatorname{Re}-187$ & $4.0 \mathrm{E}-04$ & 1.3E-04 & Th-229 & $3.7 \mathrm{E}+02$ & $1.1 \mathrm{E}+02$ \\
\hline Ir-192 & $1.3 \mathrm{E}-01$ & $4.1 \mathrm{E}-02$ & Th-230 & $1.3 E+02$ & $4.0 \mathrm{E}+01$ \\
\hline $\mathrm{Hg}-203$ & $7.9 \mathrm{E}-02$ & $2.6 \mathrm{E}-02$ & Th-231 & $6.0 \mathrm{E}-04$ & $1.8 \mathrm{E}-04$ \\
\hline Tl-207 & $2.5 \mathrm{E}-06$ & $8.4 \mathrm{E}-07$ & Th-232 & $1.9 E+02$ & $5.8 \mathrm{E}+01$ \\
\hline Tl-208 & $4.4 \mathrm{E}-04$ & $1.6 \mathrm{E}-04$ & Th-234 & $5.6 \mathrm{E}-02$ & $1.8 \mathrm{E}-02$ \\
\hline T1-209 & $1.9 \mathrm{E}-04$ & $6.7 \mathrm{E}-05$ & $\mathrm{~Pa}-231$ & $2.8 \mathrm{E}+02$ & $8.3 E+01$ \\
\hline $\mathrm{Pb}-209$ & $5.2 \mathrm{E}-05$ & $1.6 \mathrm{E}-05$ & $\mathrm{~Pa}-233$ & $2.4 \mathrm{E}-02$ & $7.8 \mathrm{E}-03$ \\
\hline $\mathrm{Pb}-210$ & $3.3 \mathrm{E}+01$ & $1.1 \mathrm{E}+01$ & $\mathrm{~Pa}-234$ & $2.0 \mathrm{E}-03$ & $6.1 \mathrm{E}-04$ \\
\hline $\mathrm{Pb}-211$ & $4.4 \mathrm{E}-03$ & $1.4 \mathrm{E}-03$ & $\mathrm{~Pa}-234 \mathrm{~m}$ & $6.8 \mathrm{E}-07$ & $2.5 \mathrm{E}-07$ \\
\hline $\mathrm{Pb}-212$ & $8.9 \mathrm{E}-02$ & $2.7 \mathrm{E}-02$ & U-232 & $2.7 \mathrm{E}+02$ & $8.0 \mathrm{E}+01$ \\
\hline $\mathrm{Pb}-214$ & $4.9 \mathrm{E}-04$ & $1.5 \mathrm{E}-04$ & U-233 & $7.5 E+01$ & $2.3 \mathrm{E}+01$ \\
\hline $\mathrm{Bi}-210$ & $1.1 \mathrm{E}-01$ & $3.3 \mathrm{E}-02$ & U-234 & $7.4 \mathrm{E}+01$ & $2.2 \mathrm{E}+01$ \\
\hline $\mathrm{Bi}-211$ & $1.6 \mathrm{E}-04$ & $5.6 \mathrm{E}-05$ & U-235 & $7.1 \mathrm{E}+01$ & $2.1 E+01$ \\
\hline $\mathrm{Bi}-212$ & $1.7 \mathrm{E}-02$ & $5.1 \mathrm{E}-03$ & U-236 & $7.0 \mathrm{E}+01$ & $2.1 \mathrm{E}+01$ \\
\hline $\mathrm{Bi}-213$ & $6.4 \mathrm{E}-04$ & $2.0 \mathrm{E}-04$ & U-237 & $7.2 \mathrm{E}-03$ & $2.3 \mathrm{E}-03$ \\
\hline $\mathrm{Bi}-214$ & $5.8 \mathrm{E}-04$ & $1.9 \mathrm{E}-04$ & U-238 & $6.6 \mathrm{E}+01$ & $2.0 \mathrm{E}+01$ \\
\hline Po-210 & $1.2 \mathrm{E}+01$ & $3.9 \mathrm{E}+00$ & $\mathrm{U}-240$ & $1.4 \mathrm{E}-03$ & 4.1E-04 \\
\hline
\end{tabular}


Table 4-11. 300 East Area--CAP88-PC Unit Dose Factors by Effective Release Height ${ }^{\text {a }}(\mathrm{mrem} / \mathrm{Ci})$.

\begin{tabular}{|c|c|c|c|c|c|}
\hline \multirow{2}{*}{ Nuclide } & \multicolumn{2}{|c|}{$\begin{array}{c}\text { Effective } \\
\text { Release Height }\end{array}$} & \multirow{2}{*}{ Nuclide } & \multicolumn{2}{|c|}{$\begin{array}{c}\text { Effective } \\
\text { Release Height }\end{array}$} \\
\hline & $\mathrm{H}_{\mathrm{eff}}<40 \mathrm{~m}$ & $\mathrm{H}_{\text {eff }} \geq 40 \mathrm{~m}$ & & $\mathrm{H}_{\mathrm{eff}}<40 \mathrm{~m}$ & $\mathrm{H}_{\text {eff }} \geq 40 \mathrm{~m}$ \\
\hline $\begin{array}{c}\text { Np-237 } \\
\text { Np-238 } \\
\text { Np-239 } \\
\text { Np-240 } \\
\text { Np-240m } \\
\text { Pu-236 } \\
\text { Pu-238 } \\
\text { Pu-239 } \\
\text { Pu-240 } \\
\text { Pu-241 }\end{array}$ & $\begin{array}{c}2.8 \mathrm{E}+02 \\
1.9 \mathrm{E}-02 \\
2.9 \mathrm{E}-03 \\
4.7 \mathrm{E}-04 \\
6.8 \mathrm{E}-05 \\
4.8 \mathrm{E}+01 \\
1.8 \mathrm{E}+02 \\
2.0 \mathrm{E}+02 \\
2.0 \mathrm{E}+02 \\
3.1 \mathrm{E}+00\end{array}$ & $\begin{array}{c}8.3 \mathrm{E}+01 \\
5.9 \mathrm{E}-03 \\
9.1 \mathrm{E}-04 \\
1.5 \mathrm{E}-04 \\
2.3 \mathrm{E}-05 \\
1.5 \mathrm{E}+01 \\
5.5 \mathrm{E}+01 \\
6.0 \mathrm{E}+01 \\
6.0 \mathrm{E}+01 \\
9.4 \mathrm{E}-01\end{array}$ & & - & \\
\hline $\begin{array}{c}\text { Pu-242 } \\
\text { Pu-243 } \\
\text { Pu-244 } \\
\text { Am-241 } \\
\text { Am-242 } \\
\text { Am-242m } \\
\text { Am-243 } \\
\text { Cm-242 } \\
\text { Cm-243 } \\
\text { Cm-244 }\end{array}$ & $\begin{array}{c}1.9 \mathrm{E}+02 \\
1.4 \mathrm{E}-04 \\
1.9 \mathrm{E}+02 \\
3.0 \mathrm{E}+02 \\
3.2 \mathrm{E}-02 \\
2.9 \mathrm{E}+02 \\
3.0 \mathrm{E}+02 \\
9.9 \mathrm{E}+00 \\
2.0 \mathrm{E}+02 \\
1.6 \mathrm{E}+02 \\
\end{array}$ & $\begin{array}{c}5.7 \mathrm{E}+01 \\
4.1 \mathrm{E}-05 \\
5.6 \mathrm{E}+01 \\
9.2 \mathrm{E}+01 \\
9.5 \mathrm{E}-03 \\
8.8 \mathrm{E}+01 \\
9.2 \mathrm{E}+01 \\
3.0 \mathrm{E}+00 \\
6.1 \mathrm{E}+01 \\
4.8 \mathrm{E}+01\end{array}$ & & & - \\
\hline $\begin{array}{c}\mathrm{Cm}-245 \\
\mathrm{Cm}-246 \\
\mathrm{Cm}-247 \\
\mathrm{Cm}-248 \\
\mathrm{Cf}-252\end{array}$ & $\begin{array}{l}3.1 \mathrm{E}+02 \\
3.1 \mathrm{E}+02 \\
2.9 \mathrm{E}+02 \\
1.1 \mathrm{E}+03 \\
8.5 \mathrm{E}+01\end{array}$ & $\begin{array}{l}9.5 \mathrm{E}+01 \\
9.3 \mathrm{E}+01 \\
8.7 \mathrm{E}+01 \\
3.4 \mathrm{E}+02 \\
2.6 \mathrm{E}+01\end{array}$ & & & \\
\hline
\end{tabular}

The effective release height, $\mathrm{x}$, is in meters.

b CAP88-PC Ver. 1.0 does not calculate doses for Be-10, Se-79, Cd-113, Cd-113m, and Gd-152. Recommend substituting the unit dose factors of the nuclides in parentheses.

c Extremely short lived nuclide, resulting in an offsite dose of zero.

d Short lived radon nuclides only provide the mechanism to circumvent the emission control device. The unit dose factors for the recommended progeny should be used instead. However, $1 \mathrm{ci}$ of $\mathrm{Rn}$ does not equal $1 \mathrm{ci}$ of $\mathrm{Pb}$. Multiply the activity of $\mathrm{Rn}-219$ by 0.0018 to get the activity of $\mathrm{Pb}-211$. Multiply the activity of Rn-220 by 0.0014 to get the activity of $\mathrm{Pb}-212$. 
Table 4-12. 300 West Area--CAP88-PC Unit Dose Factors by Effective Release Height $^{2}$ (mrem/Ci).

\begin{tabular}{|c|c|c|c|c|c|}
\hline \multirow{2}{*}{ Nuclide } & \multicolumn{2}{|c|}{$\begin{array}{c}\text { Effective } \\
\text { Release Height }\end{array}$} & \multirow{2}{*}{ Nuclide } & \multicolumn{2}{|c|}{$\begin{array}{c}\text { Effective } \\
\text { Release Height }\end{array}$} \\
\hline & $\mathrm{H}_{\text {eff }}<40 \mathrm{~m}$ & $\mathrm{H}_{\text {eff }} \geq 40 \mathrm{~m}$ & & $\mathrm{H}_{\mathrm{eff}}<40 \mathrm{~m}$ & $\mathrm{H}_{\text {eff }} \geq 40 \mathrm{~m}$ \\
\hline H-3 & $3.4 \mathrm{E}-04$ & $1.2 \mathrm{E}-04$ & $\mathrm{Kr}-85$ & $7.7 \mathrm{E}-07$ & $2.6 \mathrm{E}-07$ \\
\hline $\mathrm{Be}-7$ & 3.3E-03 & $1.2 \mathrm{E}-03$ & $\mathrm{Kr}-85 \mathrm{~m}$ & $3.6 \mathrm{E}-05$ & $1.2 \mathrm{E}-05$ \\
\hline $\mathrm{Be}-10^{\mathrm{b}}$ & $(\mathrm{Sr}-90)$ & $(\mathrm{Sr}-90)$ & $\mathrm{Kr}-87$ & $1.9 \mathrm{E}-04$ & $6.5 \mathrm{E}-05$ \\
\hline $\mathrm{C}-11$ & $1.9 \mathrm{E}-04$ & 7.0E-05 & $K r-88$ & $5.0 \mathrm{E}-04$ & $1.7 \mathrm{E}-04$ \\
\hline C-14 & $2.7 \mathrm{E}-02$ & $9.2 \mathrm{E}-03$ & $\mathrm{Kr}-89$ & $1.4 \mathrm{E}-04$ & $5.6 \mathrm{E}-05$ \\
\hline $\mathrm{C}-15^{\mathrm{c}}$ & 0.0 & 0.0 & $\mathrm{Kr}-90$ & $1.6 \mathrm{E}-06$ & $7.0 \mathrm{E}-07$ \\
\hline $\mathrm{N}-13$ & $1.4 \mathrm{E}-04$ & $5.5 \mathrm{E}-05$ & $\mathrm{Rb}-86$ & $4.8 \mathrm{E}-\mathrm{c} 2$ & $1.8 \mathrm{E}-02$ \\
\hline $0-15$ & $4.3 \mathrm{E}-05$ & $1.8 \mathrm{E}-05$ & $\mathrm{Rb}-87$ & $1.6 \mathrm{E}-01$ & $5.9 \mathrm{E}-02$ \\
\hline F-18 & $3.1 \mathrm{E}-04$ & $1.1 \mathrm{E}-04$ & $\mathrm{Rb}-88$ & $1.5 \mathrm{E}-04$ & $5.6 \mathrm{E}-05$ \\
\hline $\mathrm{Na}-22$ & $2.1 \mathrm{E}+00$ & 8.1E-01 & $\mathrm{Rb}-89$ & $3.8 \mathrm{E}-04$ & $1.4 \mathrm{E}-04$ \\
\hline $\mathrm{Na}-24$ & $3.9 \mathrm{E}-03$ & $1.4 \mathrm{E}-03$ & $R b-90$ & $1.4 \mathrm{E}-04$ & $6.0 \mathrm{E}-05$ \\
\hline P-32 & $4.8 \mathrm{E}-02$ & $1.8 \mathrm{E}-02$ & $\mathrm{Rb}-90 \mathrm{~m}$ & 3.2E-04 & $1.3 \mathrm{E}-04$ \\
\hline S-35 & $6.8 \mathrm{E}-03$ & $2.6 \mathrm{E}-03$ & Sr-89 & $2.9 \mathrm{E}-02$ & $1.1 \mathrm{E}-02$ \\
\hline $\mathrm{Ar}-41$ & $2.8 \mathrm{E}-04$ & $9.8 \mathrm{E}-05$ & Sr-90 & $1.9 \mathrm{E}+00$ & $7.1 \mathrm{E}-01$ \\
\hline$K-40$ & $2.1 E+\infty$ & $7.7 \mathrm{E}-01$ & Sr-91 & $8.4 \mathrm{E}-04$ & $3.0 \mathrm{E}-04$ \\
\hline $\mathrm{Ca}-41$ & $1.7 \mathrm{E}-04$ & $6.4 \mathrm{E}-05$ & Sr-92 & $6.7 \mathrm{E}-04$ & $2.4 \mathrm{E}-04$ \\
\hline $\mathrm{Sc}-46$ & $2.0 \mathrm{E}-01$ & $7.6 \mathrm{E}-02$ & $Y-90$ & $5.9 \mathrm{E}-03$ & $2.2 \mathrm{E}-03$ \\
\hline $\mathrm{Cr}-51$ & $1.5 \mathrm{E}-03$ & $5.5 \mathrm{E}-04$ & $Y-90 m$ & $2.2 \mathrm{E}-04$ & 8.1E-05 \\
\hline $\mathrm{Mn}-54$ & $2.7 \mathrm{E}-01$ & $1.0 \mathrm{E}-01$ & $Y-91$ & $4.3 \mathrm{E}-02$ & $1.6 \mathrm{E}-02$ \\
\hline $\mathrm{Mn}-56$ & 7.5E-04 & 2.7E-04 & Y.91m & $1.3 \mathrm{E}-04$ & $4.9 \mathrm{E}-05$ \\
\hline $\mathrm{Fe}-55$ & $3.9 \mathrm{E}-03$ & $1.4 \mathrm{E}-03$ & Y-92 & $5.4 \mathrm{E}-04$ & $1.9 \mathrm{E}-04$ \\
\hline Fe-59 & $7.9 \mathrm{E}-02$ & $3.0 \mathrm{E}-02$ & Y-93 & $1.1 \mathrm{E}-03$ & $4.0 \mathrm{E}-04$ \\
\hline Co-57 & $4.8 \mathrm{E}-02$ & $1.8 \mathrm{E}-02$ & Zr-93 & $2.2 \mathrm{E}-02$ & $7.9 \mathrm{E}-03$ \\
\hline Co-58 & $9.1 \mathrm{E}-02$ & $3.4 \mathrm{E}-02$ & Zr-95 & $6.5 \mathrm{E}-02$ & $2.5 \mathrm{E}-02$ \\
\hline Co- 60 & $4.1 \mathrm{E}+00$ & $1.6 \mathrm{E}+00$ & $\mathrm{Nb}-93 \mathrm{~m}$ & $3.5 \mathrm{E}-02$ & $1.3 \mathrm{E}-02$ \\
\hline $\mathrm{Ni}-59$ & $5.1 \mathrm{E}-03$ & $1.9 \mathrm{E}-03$ & $\mathrm{Nb}-94$ & $1.4 E+01$ & $5.1 \mathrm{E}+00$ \\
\hline $\mathrm{Ni}-63$ & $4.4 \mathrm{E}-03$ & $1.6 \mathrm{E}-03$ & $\mathrm{Nb}-95$ & $8.0 \mathrm{E}-02$ & $3.0 \mathrm{E}-02$ \\
\hline $\mathrm{Ni}-65$ & $3.0 \mathrm{E}-04$ & $1.1 \mathrm{E}-04$ & $\mathrm{Nb}-95 \mathrm{~m}$ & $5.1 \mathrm{E}-03$ & $1.9 \mathrm{E}-03$ \\
\hline $\mathrm{Cu}-64$ & $2.8 \mathrm{E}-04$ & $1.0 \mathrm{E}-04$ & Nb-97 & $2.2 \mathrm{E}-04$ & 7.9E-05 \\
\hline $\mathrm{Zn}-65$ & $4.1 \mathrm{E}-01$ & $1.6 \mathrm{E}-01$ & $\mathrm{Nb}-97 \mathrm{~m}$ & $8.3 \mathrm{E}-06$ & $3.6 \mathrm{E}-06$ \\
\hline $\mathrm{Zn}-69 \mathrm{~m}$ & $7.6 \mathrm{E}-04$ & $2.7 \mathrm{E}-04$ & Mo-93 & $4.7 \mathrm{E}-02$ & $1.8 \mathrm{E}-02$ \\
\hline $\mathrm{Zn}-69$ & 2.3E-05 & $8.3 \mathrm{E}-06$ & Mo-99 & $3.0 \mathrm{E}-03$ & $1.1 \mathrm{E}-03$ \\
\hline Ga-67 & $1.0 \mathrm{E}-03$ & $3.9 \mathrm{E}-04$ & Tc-97 & $9.6 \mathrm{E}-02$ & $3.6 \mathrm{E}-02$ \\
\hline As-76 & $2.5 \mathrm{E}-03$ & $8.8 \mathrm{E}-04$ & Tc-99 & $3.8 \mathrm{E}-01$ & $1.4 \mathrm{E}-01$ \\
\hline $\mathrm{Se}-79^{\mathrm{b}}$ & (Pu-241) & (Pu-241) & $\mathrm{Tc}-99 \mathrm{~m}$ & $7.8 \mathrm{E}-05$ & $2.8 \mathrm{E}-05$ \\
\hline $\mathrm{Br}-82$ & $5.6 \mathrm{E}-03$ & $2.1 \mathrm{E}-03$ & Tc-101 & $5.3 \mathrm{E}-05$ & $2.0 \mathrm{E}-05$ \\
\hline $\mathrm{Br}-83$ & $2.3 \mathrm{E}-06$ & $8.4 \mathrm{E}-07$ & Ru-97 & $1.1 \mathrm{E}-03$ & 4.2E-04 \\
\hline $\mathrm{Br}-84$ & $4.0 \mathrm{E}-04$ & $1.5 \mathrm{E}-04$ & $R u-103$ & $3.1 \mathrm{E}-02$ & $1.2 \mathrm{E}-02$ \\
\hline $\mathrm{Br}-85$ & $4.3 \mathrm{E}-06$ & $1.8 \mathrm{E}-06$ & $R u-105$ & $5.6 \mathrm{E}-04$ & $2.0 \mathrm{E}-04$ \\
\hline $\mathrm{Kr}-83 \mathrm{~m}$ & $5.1 \mathrm{E}-08$ & $1.8 \mathrm{E}-08$ & $\mathrm{Ru}-106$ & $2.7 \mathrm{E}-01$ & $9.6 \mathrm{E}-02$ \\
\hline
\end{tabular}


HNF-3602, Vol. 1

Table 4-12. 300 West Area--CAP88-PC Unit Dose Factors by Effective Release Height $^{a}(\mathrm{mrem} / \mathrm{Ci})$.

\begin{tabular}{|c|c|c|c|c|c|}
\hline \multirow{2}{*}{ Nuclide } & \multicolumn{2}{|c|}{$\begin{array}{c}\text { Effective } \\
\text { Release Height }\end{array}$} & \multirow{2}{*}{ Nuclide } & \multicolumn{2}{|c|}{$\begin{array}{c}\text { Effective } \\
\text { Release Height }\end{array}$} \\
\hline & $\mathrm{H}_{\mathrm{eff}}<40 \mathrm{~m}$ & $\mathrm{H}_{\text {eff }} \geq 40 \mathrm{~m}$ & & $\mathrm{H}_{\mathrm{eff}}<40 \mathrm{~m}$ & $\mathrm{H}_{\text {eff }} \geq 40 \mathrm{~m}$ \\
\hline $\mathrm{Rh}-103 \mathrm{~m}$ & $2.9 \mathrm{E}-06$ & $1.1 \mathrm{E}-06$ & I-129 & $3.6 \mathrm{E}+01$ & $2.0 \mathrm{E}+01$ \\
\hline $\mathrm{Rh}-105$ & 8.5E-04 & $3.1 \mathrm{E}-04$ & $\mathrm{I}-130$ & 1.4E-02 & $7.8 \mathrm{E}-03$ \\
\hline Rh-105m & $1.4 \mathrm{E}-07$ & $5.9 \mathrm{E}-08$ & I-131 & $1.2 \mathrm{E}+00$ & $6.5 \mathrm{E}-01$ \\
\hline Rh-106 & $1.7 \mathrm{E}-07$ & $7.6 \mathrm{E}-08$ & I-132 & $2.7 \mathrm{E}-03$ & $1.5 \mathrm{E}-03$ \\
\hline Pd-107 & $6.4 \mathrm{E}-03$ & $2.3 \mathrm{E}-03$ & I-133 & $1.4 \mathrm{E}-02$ & $7.8 \mathrm{E}-03$ \\
\hline Pd-109 & $6.6 \mathrm{E}-04$ & $2.3 \mathrm{E}-04$ & I-134 & $1.3 \mathrm{E}-03$ & 7.2E-04 \\
\hline $\mathrm{Ag}-109 \mathrm{~m}$ & $1.4 \mathrm{E}-08$ & $6.0 \mathrm{E}-09$ & I-135 & $4.9 \mathrm{E}-03$ & $2.7 \mathrm{E}-03$ \\
\hline Ag-110 & $8.5 \mathrm{E}-09$ & $3.9 \mathrm{E}-09$ & $\mathrm{Xe}-122$ & $1.5 \mathrm{E}-05$ & $5.3 \mathrm{E}-06$ \\
\hline $\mathrm{Ag}-110 \mathrm{~m}$ & $8.0 \mathrm{E}-01$ & $3.0 \mathrm{E}-01$ & $\mathrm{Xe}-123$ & $1.4 \mathrm{E}-04$ & 4.7E-05 \\
\hline $\mathrm{Ag}-111$ & $1.7 \mathrm{E}-02$ & $6.3 \mathrm{E}-03$ & $\mathrm{Xe}-125$ & $5.6 \mathrm{E}-05$ & $1.9 \mathrm{E}-05$ \\
\hline $\mathrm{Cd}-113^{b}$ & $(P u-241)$ & (Pu-241) & Xe-127 & $5.9 \mathrm{E}-05$ & $2.0 \mathrm{E}-05$ \\
\hline $\mathrm{Cd}-113 \mathrm{~m}^{\mathrm{b}}$ & (Pu-241) & (Pu-241) & $\mathrm{Xe}-131 \mathrm{~m}$ & $2.2 \mathrm{E}-06$ & $7.4 \mathrm{E}-07$ \\
\hline Cd-115 & 3.3E-03 & $1.2 \mathrm{E}-03$ & $\mathrm{Xe}-133$ & $7.9 \mathrm{E}-06$ & 2.7E-06. \\
\hline $\mathrm{Cd}-115 \mathrm{~m}$ & $6.0 \mathrm{E}-02$ & 2.2E-02 & $\mathrm{Xe}-133 \mathrm{~m}$ & $6.9 \mathrm{E}-06$ & $2.4 \mathrm{E}-06$ \\
\hline In-113m & $9.0 \mathrm{E}-05$ & $3.2 \mathrm{E}-05$ & $\mathrm{Xe}-135$ & $5.6 \mathrm{E}-05$ & $1.9 \mathrm{E}-05$ \\
\hline In-115 & $1.0 \mathrm{E}+00$ & 3.7E-01 & $\mathrm{Xe}-135 \mathrm{~m}$ & $6.7 \mathrm{E}-05$ & $2.5 \mathrm{E}-05$ \\
\hline In-115m & $1.3 \mathrm{E}-04$ & 4.7E-05 & $\mathrm{Xe}-137$ & $1.6 \mathrm{E}-05$ & $6.2 \mathrm{E}-06$ \\
\hline Sn-113 & $3.7 \mathrm{E}-02$ & $1.4 \mathrm{E}-02$ & Xe-138 & $1.9 \mathrm{E}-04$ & 7.1E-05 \\
\hline Sn-123 & $8.6 \mathrm{E}-04$ & $3.2 \mathrm{E}-04$ & Cs-134 & $1.7 \mathrm{E}+\infty 0$ & $6.3 \mathrm{E}-01$ \\
\hline Sn-125 & $4.0 \mathrm{E}-02$ & $1.5 \mathrm{E}-02$ & Cs-134m & $2.5 \mathrm{E}-05$ & $8.8 \mathrm{E}-06$ \\
\hline Sn-126 & $7.9 \mathrm{E}-01$ & $3.0 \mathrm{E}-01$ & Cs-135 & $7.4 \mathrm{E}-02$ & $2.8 \mathrm{E}-02$ \\
\hline $\mathrm{Sb}-124$ & $1.4 \mathrm{E}-01$ & $5.4 \mathrm{E}-02$ & Cs-136 & $6.2 \mathrm{E}-02$ & $2.3 \mathrm{E}-02$ \\
\hline $\mathrm{Sb}-125$ & $4.4 \mathrm{E}-01$ & $1.6 \mathrm{E}-01$ & Cs-137 & $4.4 \mathrm{E}-01$ & $1.7 \mathrm{E}-01$ \\
\hline $\mathrm{Sb}-126$ & $5.5 \mathrm{E}-02$ & $2.1 \mathrm{E}-02$ & Cs-138 & $5.4 \mathrm{E}-04$ & 2.0E-04 \\
\hline $\mathrm{Sb}-126 \mathrm{~b}$ & $2.9 \mathrm{E}-04$ & $1.1 \mathrm{E}-04$ & Cs-139 & $4.3 \mathrm{E}-05$ & 1.7E-05 \\
\hline Sb-127 & $8.0 \mathrm{E}-03$ & $2.9 \mathrm{E}-03$ & $\mathrm{Ba}-133$ & $1.3 \mathrm{E}+00$ & 4.7E-01 \\
\hline $\mathrm{Te}-125 \mathrm{~m}$ & $1.7 \mathrm{E}-02$ & $6.4 \mathrm{E}-03$ & $\mathrm{Ba}-133 \mathrm{~m}$ & 5.5E-04 & $2.0 \mathrm{E}-04$ \\
\hline $\mathrm{Te}-127$ & $1.6 \mathrm{E}-04$ & $5.8 \mathrm{E}-05$ & $\mathrm{Ba}-137 \mathrm{~m}$ & 3.3E-05 & $1.4 \mathrm{E}-05$ \\
\hline $\mathrm{Te}-127 \mathrm{~m}$ & $4.3 \mathrm{E}-02$ & $1.6 \mathrm{E}-02$ & Ba-139 & $7.7 \mathrm{E}-05$ & $2.8 \mathrm{E}-05$ \\
\hline $\mathrm{Te}-129$ & $6.0 \mathrm{E}-05$ & 2.2E-05 & Ba-140 & $1.8 \mathrm{E}-02$ & $6.6 \mathrm{E}-03$ \\
\hline $\mathrm{Te}-129 \mathrm{~m}$ & $4.2 \mathrm{E}-02$ & $1.6 \mathrm{E}-02$ & Ba-141 & $1.5 \mathrm{E}-04$ & $5.8 \mathrm{E}-05$ \\
\hline $\mathrm{Te}-131$ & $1.2 \mathrm{E}-04$ & $4.3 \mathrm{E}-05$ & $\mathrm{Ba}-142$ & $1.3 \mathrm{E}-04$ & $5.0 \mathrm{E}-05$ \\
\hline $\mathrm{Te}-13 \mathrm{im}$ & $4.2 E-03$ & $1.5 \mathrm{E}-03$ & La-140 & $7.0 \mathrm{E}-03$ & $2.6 \mathrm{E}-03$ \\
\hline $\mathrm{Te}-132$ & $7.1 \mathrm{E}-03$ & $2.6 \mathrm{E}-03$ & La-141 & $1.6 \mathrm{E}-05$ & $5.7 \mathrm{E}-06$ \\
\hline $\mathrm{Te}-133$ & $1.4 \mathrm{E}-04$ & $5.4 \mathrm{E}-05$ & $\mathrm{La}-142$ & $8.0 \mathrm{E}-04$ & $2.9 \mathrm{E}-04$ \\
\hline Te-133m & 5.3E-04 & $1.9 \mathrm{E}-04$ & $\mathrm{Ce}-141$ & $1.3 \mathrm{E}-02$ & $4.7 \mathrm{E}-03$ \\
\hline $\mathrm{Te}-134$ & $1.9 \mathrm{E}-04$ & $6.9 \mathrm{E}-05$ & Ce-143 & $2.1 \mathrm{E}-03$ & 7.5E-04 \\
\hline I-122 & $5.4 \mathrm{E}-05$ & $3.4 \mathrm{E}-05$ & Ce-144 & $2.1 \mathrm{E}-01$ & $7.6 \mathrm{E}-02$ \\
\hline I-123 & $1.3 \mathrm{E}-03$ & 7.1E-04 & Pr-143 & $1.0 \mathrm{E}-02$ & $3.7 \mathrm{E}-03$ \\
\hline $\mathrm{I}-125$ & $2.1 \mathrm{E}+00$ & $1.2 \mathrm{E}+00$ & Pr-144 & $2.8 \mathrm{E}-05$ & $1.0 \mathrm{E}-05$ \\
\hline
\end{tabular}


Table 4-12. 300 West Area--CAP88-PC Unit Dose Factors by Effective Release Height $^{\mathrm{a}}$ (mrem/Ci).

\begin{tabular}{|c|c|c|c|c|c|}
\hline \multirow{2}{*}{ Nuclide } & \multicolumn{2}{|c|}{$\begin{array}{c}\text { Effective } \\
\text { Release Height }\end{array}$} & \multirow{2}{*}{ Nuclide } & \multicolumn{2}{|c|}{$\begin{array}{c}\text { Effective } \\
\text { Release Height }\end{array}$} \\
\hline & $\mathrm{H}_{\mathrm{eff}}<40 \mathrm{~m}$ & $\mathrm{H}_{\mathrm{eff}} \geq 40 \mathrm{~m}$ & & $\mathrm{H}_{\text {eff }}<40 \mathrm{~m}$ & $\mathrm{H}_{\text {eff }} \geq 40 \mathrm{~m}$ \\
\hline $\mathrm{Pr}-144 \mathrm{~m}$ & $7.4 \mathrm{E}-06$ & $2.9 \mathrm{E}-06$ & Po- $211^{\mathrm{c}}$ & 0.0 & 0.0 \\
\hline Nd-147 & $9.8 \mathrm{E}-03$ & $3.6 \mathrm{E}-03$ & $\mathrm{Po}-212^{\mathrm{c}}$ & 0.0 & 0.0 \\
\hline Pm-147 & $1.8 \mathrm{E}-02$ & $6.6 \mathrm{E}-03$ & Po- $213^{c}$ & 0.0 & 0.0 \\
\hline Pm-148 & $1.5 \mathrm{E}-02$ & $5.4 \mathrm{E}-03$ & Po-214 & 0.0 & 0.0 \\
\hline Pm-148m & $1.3 \mathrm{E}-01$ & $4.9 \mathrm{E}-02$ & $\mathrm{Po}-215^{\mathrm{c}}$ & 0.0 & 0.0 \\
\hline Pm-149 & $1.9 \mathrm{E}-03$ & $6.8 \mathrm{E}-04$ & Po- $216^{\mathrm{c}}$ & 0.0 & 0.0 \\
\hline Pm-151 & $5.0 \mathrm{E}-04$ & $1.9 \mathrm{E}-04$ & Po-218 & $5.9 \mathrm{E}-06$ & $2.5 \mathrm{E}-06$ \\
\hline Sm-147 & $2.9 \mathrm{E}+01$ & $1.0 \mathrm{E}+01$ & At $-217^{\mathrm{c}}$ & 0.0 & 0.0 \\
\hline Sm-151 & $1.3 \mathrm{E}-02$ & $4.5 \mathrm{E}-03$ & $R n-219^{d}$ & $(\mathrm{~Pb}-211)$ & $(\mathrm{Pb}-211)$ \\
\hline Sm-153 & $1.3 \mathrm{E}-03$ & $4.8 \mathrm{E}-04$ & $R n-220^{d}$ & $(\mathrm{~Pb}-212)$ & $(\mathrm{Pb}-212)$ \\
\hline $\mathrm{Eu}-152$ & $4.0 \mathrm{E}+00$ & $1.5 E+00$ & $\mathrm{Rn}-222$ & $9.7 \mathrm{E}-04$ & $3.3 \mathrm{E}-04$ \\
\hline $\mathrm{Eu}-152 \mathrm{~m}$ & $1.9 \mathrm{E}-04$ & $7.1 \mathrm{E}-05$ & Fr-221 & $6.9 \mathrm{E}-04$ & $2.8 \mathrm{E}-04$ \\
\hline Eu-154 & $3.3 \mathrm{E}+00$ & $1.2 \mathrm{E}+\infty$ & Fr-223 & $8.0 \mathrm{E}-04$ & $3.0 \mathrm{E}-04$ \\
\hline Eu-155 & $1.3 \mathrm{E}-01$ & $5.0 \mathrm{E}-02$ & $\mathrm{Ra}-223$ & $4.1 \mathrm{E}+00$ & $1.5 \mathrm{E}+00$ \\
\hline Eu-156 & $4.1 E-02$ & $1.5 \mathrm{E}-02$ & $\mathrm{Ra}-224$ & $1.5 \mathrm{E}+00$ & $5.3 \mathrm{E}-01$ \\
\hline $\mathrm{Gd}-152^{\mathrm{b}}$ & (Pu-239) & (Pu-239) & $\mathrm{Ra}-225$ & $2.5 \mathrm{E}+00$ & $9.1 \mathrm{E}-01$ \\
\hline $\mathrm{Tb}-160$ & $1.1 \mathrm{E}-01$ & $4.0 \mathrm{E}-02$ & $\mathrm{Ra}-226$ & $7.8 \mathrm{E}+00$ & $2.8 \mathrm{E}+00$ \\
\hline Ho-166 & $1.6 \mathrm{E}-03$ & $5.7 \mathrm{E}-04$ & $\mathrm{Ra}-228$ & $3.2 E+00$ & $1.2 \mathrm{E}+\infty 0$ \\
\hline Ho-166m & $1.3 \mathrm{E}+01$ & $5.0 \mathrm{E}+00$ & Ac-225 & $2.4 \mathrm{E}+00$ & $8.5 \mathrm{E}-01$ \\
\hline Hf-181 & $4.1 \mathrm{E}-02$ & $1.5 \mathrm{E}-02$ & $\mathrm{Ac}-227$ & $2.5 \mathrm{E}+02$ & $8.9 \mathrm{E}+01$ \\
\hline W-181 & $7.5 \mathrm{E}-03$ & $2.8 \mathrm{E}-03$ & Ac-228 & $3.2 \mathrm{E}-02$ & $1.1 \mathrm{E}-02$ \\
\hline W-185 & $1.0 \mathrm{E}-02$ & $3.8 \mathrm{E}-03$ & Th-227 & $4.5 \mathrm{E}+\infty 0$ & $1.6 \mathrm{E}+00$ \\
\hline W-187 & 9.1E-04 & $3.3 \mathrm{E}-04$ & Th-228 & $9.6 \mathrm{E}+01$ & $3.4 \mathrm{E}+01$ \\
\hline $\operatorname{Re}-187$ & $2.9 \mathrm{E}-04$ & $1.1 \mathrm{E}-04$ & Th-229 & $2.7 \mathrm{E}+02$ & $9.4 E+01$ \\
\hline Ir -192 & $9.1 \mathrm{E}-02$ & $3.4 \mathrm{E}-02$ & Th-230 & $9.6 \mathrm{E}+01$ & $3.4 \mathrm{E}+01$ \\
\hline $\mathrm{Hg}-203$ & $5.7 \mathrm{E}-02$ & 2.1E-02 & Th-231 & 4.3E-04 & $1.5 \mathrm{E}-04$ \\
\hline Tl-207 & $1.5 \mathrm{E}-06$ & $6.1 \mathrm{E}-07$ & Th-232 & $1.4 \mathrm{E}+02$ & $4.8 \mathrm{E}+01$ \\
\hline T1-208 & $2.6 \mathrm{E}-04$ & $1.1 \mathrm{E}-04$ & Th-234 & $4.0 \mathrm{E}-02$ & $1.5 \mathrm{E}-02$ \\
\hline Tl-209 & $1.0 \mathrm{E}-04$ & $4.3 \mathrm{E}-05$ & $\mathrm{~Pa}-231$ & $2.0 \mathrm{E}+02$ & $7.0 \mathrm{E}+01$ \\
\hline $\mathrm{Pb}-209$ & $3.7 \mathrm{E}-05$ & $1.3 \mathrm{E}-05$ & $\mathrm{~Pa}-233$ & $1.8 \mathrm{E}-02$ & $6.5 \mathrm{E}-03$ \\
\hline $\mathrm{Pb}-210$ & $2.4 \mathrm{E}+01$ & $8.8 \mathrm{E}+00$ & $\mathrm{~Pa}-234$ & $1.4 \mathrm{E}-03$ & $5.1 \mathrm{E}-04$ \\
\hline $\mathrm{Pb}-211$ & $3.1 \mathrm{E}-03$ & $1.1 \mathrm{E}-03$ & $\mathrm{~Pa}-234 \mathrm{~m}$ & $3.0 \mathrm{E}-07$ & $1.3 \mathrm{E}-07$ \\
\hline $\mathrm{Pb}-212$ & $6.4 \mathrm{E}-02$ & $2.2 \mathrm{E}-02$ & $\mathrm{U}-232$ & $1.9 E+02$ & $6.7 \mathrm{E}+01$ \\
\hline $\mathrm{Pb}-214$ & $3.3 \mathrm{E}-04$ & $1.2 \mathrm{E}-04$ & U-233 & $5.4 \mathrm{E}+01$ & $1.9 \mathrm{E}+01$ \\
\hline $\mathrm{Bi}-210$ & $7.8 \mathrm{E}-02$ & 2.7E-02 & U-234 & $5.3 E+01$ & $1.9 E+01$ \\
\hline $\mathrm{Bi}-211$ & $8.6 \mathrm{E}-05$ & $3.6 \mathrm{E}-05$ & U-235 & $5.1 E+01$ & $1.8 \mathrm{E}+01$ \\
\hline $\mathrm{Bi}-212$ & $1.2 \mathrm{E}-02$ & $4.2 \mathrm{E}-03$ & U-236 & $5.0 \mathrm{E}+01$ & $1.8 \mathrm{E}+01$ \\
\hline $\mathrm{Bi}-213$ & $4.4 \mathrm{E}-04$ & $1.6 \mathrm{E}-04$ & U-237 & $5.2 \mathrm{E}-03$ & $1.9 \mathrm{E}-03$ \\
\hline $\mathrm{Bi}-214$ & $3.9 \mathrm{E}-04$ & $1.5 \mathrm{E}-04$ & U-238 & $4.7 \mathrm{E}+01$ & $1.7 \mathrm{E}+01$ \\
\hline Po-210 & $8.8 \mathrm{E}+00$ & $3.2 \mathrm{E}+00$ & U-240 & $9.6 \mathrm{E}-04$ & $3.4 \mathrm{E}-04$ \\
\hline
\end{tabular}


HNF-3602, Vol. 1

Table 4-12. 300 West Area--CAP88-PC Unit Dose Factors by Effective Release Height ${ }^{\mathrm{a}}$ (mrem/Ci).

\begin{tabular}{|c|c|c|c|c|c|}
\hline \multirow{2}{*}{ Nuclide } & \multicolumn{2}{|c|}{$\begin{array}{c}\text { Effective } \\
\text { Release Height }\end{array}$} & \multirow{2}{*}{ Nuclide } & \multicolumn{2}{|c|}{$\begin{array}{c}\text { Effective } \\
\text { Release Height }\end{array}$} \\
\hline & $\mathrm{H}_{\mathrm{eff}}<40 \mathrm{~m}$ & $\mathrm{H}_{\text {eff }} \geq 40 \mathrm{~m}$ & & $\mathrm{H}_{\mathrm{eff}}<40 \mathrm{~m}$ & $\mathrm{H}_{\text {eff }} \geq 40 \mathrm{~m}$ \\
\hline Np-237 & $2.0 \mathrm{E}+02$ & $7.0 \mathrm{E}+01$ & & & \\
\hline Np-238 & $1.4 \mathrm{E}-02$ & $4.9 E-03$ & & & \\
\hline Np-239 & $2.1 \mathrm{E}-03$ & $7.6 \mathrm{E}-04$ & & & \\
\hline $\mathrm{Np}-240$ & $3.3 \mathrm{E}-04$ & $1.2 \mathrm{E}-04$ & & & \\
\hline $\mathrm{Np}-240 \mathrm{~m}$ & $4.3 \mathrm{E}-05$ & $1.7 \mathrm{E}-05$ & & & \\
\hline Pu-236 & $3.5 \mathrm{E}+01$ & $1.2 \mathrm{E}+01$ & & & \\
\hline Pul-238 & $1.3 E+02$ & $4.6 \mathrm{E}+01$ & & & \\
\hline Pu-239 & $1.4 \mathrm{E}+02$ & $5.0 \mathrm{E}+01$ & & & \\
\hline$P u-240$ & $1.4 \mathrm{E}+02$ & $5.0 \mathrm{E}+01$ & & & \\
\hline Pu-241 & $2.2 \mathrm{E}+00$ & $7.9 \mathrm{E}-01$ & & & \\
\hline $\mathrm{Pu}-242$ & $1.4 \mathrm{E}+02$ & $4.8 \mathrm{E}+01$ & & & \\
\hline Pu-243 & $9.6 \mathrm{E}-05$ & $3.4 \mathrm{E}-05$ & & & \\
\hline Pu-244 & $1.3 \mathrm{E}+02$ & $4.7 \mathrm{E}+01$ & & & \\
\hline Am-241 & $2.2 \mathrm{E}+02$ & $7.7 \mathrm{E}+01$ & & & \\
\hline Am-242 & $2.3 \mathrm{E}-02$ & $7.9 \mathrm{E}-03$ & & & \\
\hline Am-242m & $2.1 E+02$ & $7.4 \mathrm{E}+01$ & & & \\
\hline Am-243 & $2.2 \mathrm{E}+02$ & $7.7 \mathrm{E}+01$ & & & \\
\hline $\mathrm{Cm}-242$ & $7.1 E+\infty$ & $2.5 \mathrm{E}+\infty 0$ & & & \\
\hline $\mathrm{Cm}-243$ & $1.5 \mathrm{E}+02$ & $5.2 \mathrm{E}+01$ & & & \\
\hline $\mathrm{Cm}-244$ & $1.2 \mathrm{E}+02$ & 4.1E+01 & & & \\
\hline $\mathrm{Cm}-245$ & $2.3 \mathrm{E}+02$ & $7.9 \mathrm{E}+01$ & & & \\
\hline $\mathrm{Cm}-246$ & $2.2 E+02$ & $7.8 \mathrm{E}+01$ & & & \\
\hline $\mathrm{Cm}-247$ & $2.1 \mathrm{E}+02$ & $7.3 \mathrm{E}+01$ & & & \\
\hline $\mathrm{Cm}-248$ & $8.2 E+02$ & $2.9 \mathrm{E}+02$ & & & \\
\hline Cf-252 & $6.1 E+01$ & $2.2 \mathrm{E}+01$ & & & \\
\hline
\end{tabular}

a The effective release height, $x$, is in meters.

- CAP88-PC Ver. 1.0 does not calculate doses for Be-10, Se-79, Cd-113, Cd-113m, and Gd-152. Recommend substituting the unit dose factors of the nuclides in parentheses.

c Extremely short lived nuclide, resulting in an offsite dose of zero.

- Short lived radon nuclides only provide the mechanism to circumvent the emission control device. The unit dose factors for the recommended progeny should be used instead. However, 1 ci of $\mathrm{Rn}$ does not equal $1 \mathrm{ci}$ of $\mathrm{Pb}$. Multiply the activity of Rn-219 by 0.0018 to get the activity of $\mathrm{Pb}-211$. Multiply the activity of $\mathrm{Rn}-220$ by 0.0014 to get the activity of $\mathrm{Pb}-212$. 
Table 4-13. 400 Area--CAP88-PC Unit Dose Factors by Effective Release Height ${ }^{a}$ (mrem/Ci).

\begin{tabular}{|c|c|c|c|c|c|}
\hline \multirow{2}{*}{ Nuclide } & \multicolumn{2}{|c|}{$\begin{array}{c}\text { Effective } \\
\text { Release Height }\end{array}$} & \multirow{2}{*}{ Nuclide } & \multicolumn{2}{|c|}{$\begin{array}{c}\text { Effective } \\
\text { Release Height }\end{array}$} \\
\hline & $\mathrm{H}_{\mathrm{eff}}<40 \mathrm{~m}$ & $\mathrm{H}_{\text {eff }} \geq 40 \mathrm{~m}$ & & $\mathrm{H}_{\mathrm{eff}}<40 \mathrm{~m}$ & $\mathrm{H}_{\text {eff }} \geq 40 \mathrm{~m}$ \\
\hline $\mathrm{H}-3$ & $3.4 \mathrm{E}-05$ & $1.9 \mathrm{E}-05$ & $\mathrm{Kr}-85$ & $7.5 \mathrm{E}-08$ & $4.2 \mathrm{E}-08$ \\
\hline $\mathrm{Be}-7$ & $2.9 \mathrm{E}-04$ & $1.9 \mathrm{E}-04$ & $\mathrm{Kr}-85 \mathrm{~m}$ & $3.1 \mathrm{E}-06$ & $1.8 \mathrm{E}-06$ \\
\hline $\mathrm{Be}-10^{\mathrm{b}}$ & $(\mathrm{Sr}-90)$ & $(\mathrm{Sr}-90)$ & $\mathrm{Kr}-87$ & $1.3 \mathrm{E}-05$ & $7.3 \mathrm{E}-06$ \\
\hline C-11 & 5.3E-06 & 3.7E-06 & $\mathrm{Kr}-88$ & $4.0 \mathrm{E}-05$ & 2.3E-05 \\
\hline C-14 & $2.6 \mathrm{E}-03$ & $1.5 \mathrm{E}-03$ & $\mathrm{Kr}-89$ & 5.3E-08 & 3.7E-08 \\
\hline $\mathrm{C}-15^{\mathrm{c}}$ & 0.0 & 0.0 & $\mathrm{Kr}-90$ & $6.1 \mathrm{E}-20$ & $4.1 \mathrm{E}-20$ \\
\hline $\mathrm{N}-13$ & $1.6 \mathrm{E}-06$ & $1.2 \mathrm{E}-06$ & $\mathrm{Rb}-86$ & $4.2 \mathrm{E}-\mathrm{C} 3$ & $2.8 \mathrm{E}-03$ \\
\hline $0-15$ & $1.0 \mathrm{E}-09$ & $7.7 \mathrm{E}-10$ & $\mathrm{Rb}-87$ & $1.4 \mathrm{E}-02$ & $9.2 \mathrm{E}-03$ \\
\hline F-18 & $2.0 \mathrm{E}-05$ & $1.3 \mathrm{E}-05$ & $\mathrm{Rb}-88$ & $3.7 \mathrm{E}-06$ & $2.6 \mathrm{E}-06$ \\
\hline $\mathrm{Na}-22$ & $1.9 \mathrm{E}-01$ & $1.3 \mathrm{E}-01$ & $\mathrm{Rb}-89$ & $8.0 \mathrm{E}-06$ & $5.6 \mathrm{E}-06$ \\
\hline $\mathrm{Na}-24$ & $3.3 \mathrm{E}-04$ & $2.1 \mathrm{E}-04$ & Rb-90 & $1.8 \mathrm{E}-08$ & $1.3 \mathrm{E}-08$ \\
\hline P-32 & $4.3 \mathrm{E}-03$ & $2.8 \mathrm{E}-03$ & $\mathrm{Rb}-90 \mathrm{~m}$ & $4.0 \mathrm{E}-07$ & 3.0E-07 \\
\hline S-35 & $6.0 \mathrm{E}-04$ & $4.0 \mathrm{E}-04$ & Sr-89 & $2.5 \mathrm{E}-03$ & $1.7 \mathrm{E}-03$ \\
\hline Ar-41 & $2.1 \mathrm{E}-05$ & $1.2 \mathrm{E}-05$ & Sr-90 & 1.7E-01 & $1.1 \mathrm{E}-01$ \\
\hline $\mathrm{K}-40$ & $1.8 \mathrm{E}-01$ & $1.2 \mathrm{E}-01$ & Sr-91 & $6.9 \mathrm{E}-05$ & $4.4 \mathrm{E}-05$ \\
\hline $\mathrm{Ca}-41$ & $1.5 \mathrm{E}-05$ & $9.9 \mathrm{E}-06$ & Sr-92 & 4.7E-05 & $3.1 \mathrm{E}-05$ \\
\hline Sc-46 & $1.8 \mathrm{E}-02$ & $1.2 \mathrm{E}-02$ & $Y-90$ & $5.1 \mathrm{E}-04$ & $3.3 \mathrm{E}-04$ \\
\hline $\mathrm{Cr}-51$ & $1.3 \mathrm{E}-04$ & 8.5E-05 & Y.90m & $1.6 \mathrm{E}-05$ & $1.1 \mathrm{E}-05$ \\
\hline $\mathrm{Mn}-54$ & $2.4 \mathrm{E}-02$ & $1.6 \mathrm{E}-02$ & Y-91 & 3.7E-03 & $2.4 \mathrm{E}-03$ \\
\hline$M n-56$ & $5.3 \mathrm{E}-05$ & $3.4 \mathrm{E}-05$ & $\mathrm{Y}-91 \mathrm{~m}$ & $6.7 \mathrm{E}-06$ & 4.4E-06 \\
\hline $\mathrm{Fe}-55$ & $3.4 \mathrm{E}-04$ & $2.2 \mathrm{E}-04$ & Y-92 & $3.9 \mathrm{E}-05$ & $2.5 \mathrm{E}-05$ \\
\hline $\mathrm{Fe}-59$ & $6.9 \mathrm{E}-03$ & $4.6 \mathrm{E}-03$ & Y-93 & 9.1E-05 & $5.8 \mathrm{E}-05$ \\
\hline Co-57 & $4.2 \mathrm{E}-03$ & $2.8 \mathrm{E}-03$ & Zr-93 & $1.9 \mathrm{E}-03$ & $1.2 \mathrm{E}-03$ \\
\hline $\mathrm{Co}-58$ & 8.0E-03 & $5.3 \mathrm{E}-03$ & $\mathrm{Zr}-95$ & $5.7 \mathrm{E}-03$ & $3.8 \mathrm{E}-03$ \\
\hline Co- 60 & $3.6 \mathrm{E}-01$ & $2.4 \mathrm{E}-01$ & $\mathrm{Nb}-93 \mathrm{~m}$ & $3.0 \mathrm{E}-03$ & $2.0 \mathrm{E}-03$ \\
\hline $\mathrm{Ni}-59$ & $4.5 \mathrm{E}-04$ & $3.0 \mathrm{E}-04$ & $\mathrm{Nb}-94$ & $1.2 \mathrm{E}+\infty$ & 7.9E-01 \\
\hline $\mathrm{Ni}-63$ & $3.8 \mathrm{E}-04$ & $2.5 \mathrm{E}-04$ & $\mathrm{Nb}-95$ & $7.0 \mathrm{E}-03$ & $4.7 \mathrm{E}-03$ \\
\hline $\mathrm{Ni}-65$ & $2.1 \mathrm{E}-05$ & $1.4 \mathrm{E}-05$ & $\mathrm{Nb}-95 \mathrm{~m}$ & $4.4 \mathrm{E}-04$ & $2.9 \mathrm{E}-04$ \\
\hline $\mathrm{Cu}-64$ & $2.3 \mathrm{E}-05$ & $1.5 \mathrm{E}-05$ & $\mathrm{Nb}-97$ & $1.3 \mathrm{E}-05$ & $8.3 \mathrm{E}-06$ \\
\hline $\mathrm{Zn}-65$ & $3.6 \mathrm{E}-02$ & $2.4 \mathrm{E}-02$ & $\mathrm{Nb}-97 \mathrm{~m}$ & $9.8 \mathrm{E}-14$ & 7.3E-14 \\
\hline $\mathrm{Zn}-69 \mathrm{~m}$ & $6.3 \mathrm{E}-05$ & $4.0 \mathrm{E}-05$ & Mo-93 & $4.1 \mathrm{E}-03$ & $2.7 \mathrm{E}-03$ \\
\hline $\mathrm{Zn}-69$ & $1.2 \mathrm{E}-06$ & $7.9 \mathrm{E}-07$ & Mo-99 & $2.6 \mathrm{E}-04$ & $1.7 \mathrm{E}-04$ \\
\hline Ga-67 & $9.0 \mathrm{E}-05$ & $5.9 \mathrm{E}-05$ & Tc-97 & $8.4 \mathrm{E}-03$ & $5.6 \mathrm{E}-03$ \\
\hline As-76 & $2.1 \mathrm{E}-04$ & $1.3 \mathrm{E}-04$ & Tc-99 & $3.3 \mathrm{E}-02$ & $2.2 \mathrm{E}-02$ \\
\hline $\mathrm{Se}-79^{\mathrm{b}}$ & (Pu-241) & (Pu-241) & Tc-99m & $6.2 \mathrm{E}-06$ & $4.0 \mathrm{E}-06$ \\
\hline $\mathrm{Br}-82$ & $4.8 \mathrm{E}-04$ & $3.1 \mathrm{E}-04$ & Tc-101 & $1.0 \mathrm{E}-06$ & $7.2 \mathrm{E}-07$ \\
\hline $\mathrm{Br}-83$ & $1.6 \mathrm{E}-07$ & $1.0 \mathrm{E}-07$ & Ru-97 & $9.8 \mathrm{E}-05$ & $6.4 \mathrm{E}-05$ \\
\hline $\mathrm{Br}-84$ & $1.6 \mathrm{E}-05$ & $1.1 \mathrm{E}-05$ & Ru-103 & $2.7 \mathrm{E}-03$ & $1.8 \mathrm{E}-03$ \\
\hline $\mathrm{Br}-85$ & $8.8 \mathrm{E}-10$ & $6.6 \mathrm{E}-10$ & Ru-105 & $4.3 \mathrm{E}-05$ & $2.8 \mathrm{E}-05$ \\
\hline $\mathrm{Kr}-83 \mathrm{~m}$ & $3.8 \mathrm{E}-09$ & 2.2E-09 & $\mathrm{Ru}-106$ & 2.3E-02 & $1.5 \mathrm{E}-02$ \\
\hline
\end{tabular}


HNF-3602, Vol. 1

Table 4-13. 400 Area--CAP88-PC Unit Dose Factors by Effective Release Height ${ }^{a}$ (mrem/Ci).

\begin{tabular}{|c|c|c|c|c|c|}
\hline \multirow{2}{*}{ Nuclide } & \multicolumn{2}{|c|}{$\begin{array}{c}\text { Effective } \\
\text { Release Height }\end{array}$} & \multirow{2}{*}{ Nuclide } & \multicolumn{2}{|c|}{$\begin{array}{c}\text { Effective } \\
\text { Release Height }\end{array}$} \\
\hline & $\mathrm{H}_{\mathrm{eff}}<40 \mathrm{~m}$ & $\mathrm{H}_{\text {eff }} \geq 40 \mathrm{~m}$ & & $\mathrm{H}_{\mathrm{eff}}<40 \mathrm{~m}$ & $\mathrm{H}_{\mathrm{eff}} \geq 40 \mathrm{~m}$ \\
\hline $\mathrm{Rh}-103 \mathrm{~m}$ & $1.5 \mathrm{E}-07$ & $1.0 \mathrm{E}-07$ & I-129 & $6.2 \mathrm{E}-01$ & $1.3 \mathrm{E}+00$ \\
\hline $\mathrm{Rh}-105$ & $7.2 \mathrm{E}-05$ & 4.7E-05 & $\mathrm{I}-130$ & $2.3 \mathrm{E}-04$ & $5.0 \mathrm{E}-04$ \\
\hline $\mathrm{Rh}-105 \mathrm{~m}$ & $1.1 \mathrm{E}-17$ & $8.2 \mathrm{E}-18$ & I-131 & $2.0 \mathrm{E}-02$ & $4.4 \mathrm{E}-02$ \\
\hline Rh-106 & $6.3 \mathrm{E}-22$ & $4.6 \mathrm{E}-22$ & $\mathrm{I}-132$ & $3.9 \mathrm{E}-05$ & $8.2 \mathrm{E}-05$ \\
\hline Pd-107 & $5.5 \mathrm{E}-04$ & $3.5 \mathrm{E}-04$ & I-133 & $2.4 \mathrm{E}-04$ & $5.1 \mathrm{E}-04$ \\
\hline Pd-109 & $5.4 \mathrm{E}-05$ & $3.4 \mathrm{E}-05$ & I-134 & $1.4 \mathrm{E}-05$ & $3.0 \mathrm{E}-05$ \\
\hline $\mathrm{Ag}-109 \mathrm{~m}$ & $7.4 \mathrm{E}-20$ & $5.5 \mathrm{E}-20$ & I-135 & $7.9 \mathrm{E}-05$ & $1.7 \mathrm{E}-04$ \\
\hline Ag-110 & $4.8 \mathrm{E}-26$ & $3.5 \mathrm{E}-26$ & $\mathrm{Xe}-122$ & $1.5 \mathrm{E}-06$ & $8.2 \mathrm{E}-07$ \\
\hline $\mathrm{Ag}-110 \mathrm{~m}$ & $7.1 \mathrm{E}-02$ & $4.7 \mathrm{E}-02$ & $\mathrm{Xe}-123$ & $1.1 \mathrm{E}-05$ & $6.1 \mathrm{E}-06$ \\
\hline Ag-111 & $1.5 \mathrm{E}-03$ & $9.8 \mathrm{E}-04$ & $\mathrm{Xe}-125$ & $5.3 \mathrm{E}-06$ & $3.0 \mathrm{E}-06$ \\
\hline $\mathrm{Cd}-113^{\mathrm{b}}$ & (Pu-241) & $(\mathrm{Pu}-241)$ & $\mathrm{Xe}-127$ & $5.8 \mathrm{E}-06$ & $3.3 \mathrm{E}-06$ \\
\hline $\mathrm{Cd}-113 \mathrm{~m}^{\mathrm{b}}$ & (Pu-241) & (Pu-241) & $\mathrm{Xe}-131 \mathrm{~m}$ & $2.1 \mathrm{E}-07$ & 1.2E-07 \\
\hline Cd-115 & $2.9 \mathrm{E}-04$ & $1.9 \mathrm{E}-04$ & $\mathrm{Xe}-133$ & $7.8 \mathrm{E}-07$ & 4.4E-07 \\
\hline Cd-115m & $5.3 \mathrm{E}-03$ & $3.4 \mathrm{E}-03$ & $\mathrm{Xe}-133 \mathrm{~m}$ & $6.7 \mathrm{E}-07$ & $3.8 \mathrm{E}-07$ \\
\hline $\mathrm{In}-113 \mathrm{~m}$ & 5.7E-06 & $3.7 \mathrm{E}-06$ & $\mathrm{Xe}-135$ & $5.1 \mathrm{E}-06$ & $2.9 \mathrm{E}-06$ \\
\hline $\ln -115$ & $8.8 \mathrm{E}-02$ & $5.8 \mathrm{E}-02$ & $\mathrm{Xe}-135 \mathrm{~m}$ & $1.6 \mathrm{E}-06$ & $1.0 \mathrm{E}-06$ \\
\hline $\mathrm{In}-115 \mathrm{~m}$ & $9.9 \mathrm{E}-06$ & $6.4 \mathrm{E}-06$ & $\mathrm{Xe}-137$ & $1.4 \mathrm{E}-08$ & $9.7 \mathrm{E}-09$ \\
\hline $\mathrm{Sn}-113$ & 3.3E-03 & $2.2 \mathrm{E}-03$ & $\mathrm{Xe}-138$ & $4.1 \mathrm{E}-06$ & $2.6 \mathrm{E}-06$ \\
\hline Sn-123 & $7.6 \mathrm{E}-05$ & $5.0 \mathrm{E}-05$ & Cs-134 & $1.5 \mathrm{E}-01$ & $9.7 \mathrm{E}-02$ \\
\hline Sn-125 & $3.5 \mathrm{E}-03$ & $2.3 \mathrm{E}-03$ & Cs $-134 m$ & $1.8 \mathrm{E}-06$ & $1.1 \mathrm{E}-06$ \\
\hline $\mathrm{Sn}-126$ & $6.9 \mathrm{E}-02$ & $4.6 \mathrm{E}-02$ & Cs-135 & $6.5 \mathrm{E}-03$ & $4.3 \mathrm{E}-03$ \\
\hline $\mathrm{Sb}-124$ & $1.3 \mathrm{E}-02$ & $8.4 \mathrm{E}-03$ & Cs-136 & $5.4 \mathrm{E}-03$ & $3.6 \mathrm{E}-03$ \\
\hline $\mathrm{Sb}-125$ & $3.8 \mathrm{E}-02$ & $2.6 \mathrm{E}-02$ & Cs- 137 & $3.9 \mathrm{E}-02$ & $2.6 \mathrm{E}-02$ \\
\hline $\mathrm{Sb}-126$ & $4.8 \mathrm{E}-03$ & $3.2 \mathrm{E}-03$ & Cs- 138 & 2.2E-05 & $1.5 \mathrm{E}-05$ \\
\hline $\mathrm{Sb}-126 \mathrm{~b}$ & 7.7E-06 & $5.3 \mathrm{E}-06$ & Cs-139 & $4.5 \mathrm{E}-07$ & $3.3 \mathrm{E}-07$ \\
\hline $\mathrm{Sb}-127$ & $6.9 \mathrm{E}-04$ & $4.5 \mathrm{E}-04$ & $\mathrm{Ba}-133$ & $1.1 \mathrm{E}-01$ & $7.3 \mathrm{E}-02$ \\
\hline $\mathrm{Te}-125 \mathrm{~m}$ & $1.5 \mathrm{E}-03$ & $1.0 \mathrm{E}-03$ & $\mathrm{Ba}-133 \mathrm{~m}$ & 4.7E-05 & $3.1 \mathrm{E}-05$ \\
\hline $\mathrm{Te}-127$ & $1.3 \mathrm{E}-05$ & $8.4 \mathrm{E}-06$ & $\mathrm{Ba}-137 \mathrm{~m}$ & $3.6 \mathrm{E}-09$ & $2.7 \mathrm{E}-09$ \\
\hline $\mathrm{Te}-127 \mathrm{~m}$ & $3.7 \mathrm{E}-03$ & $2.5 \mathrm{E}-03$ & Ba-139 & $4.6 \mathrm{E}-06$ & $3.0 \mathrm{E}-06$ \\
\hline $\mathrm{Te}-129$ & $3.4 \mathrm{E}-06$ & $2.2 \mathrm{E}-06$ & Ba-140 & $1.6 \mathrm{E}-03$ & $1.0 \mathrm{E}-03$ \\
\hline $\mathrm{Te}-129 \mathrm{~m}$ & $3.7 \mathrm{E}-03$ & $2.4 \mathrm{E}-03$ & Ba-141 & $3.9 \mathrm{E}-06$ & $2.7 \mathrm{E}-06$ \\
\hline $\mathrm{Te}-131$ & $3.9 \mathrm{E}-06$ & $2.7 \mathrm{E}-06$ & $\mathrm{Ba}-142$ & $1.7 \mathrm{E}-06$ & $1.2 \mathrm{E}-06$ \\
\hline $\mathrm{Te}-131 \mathrm{~m}$ & $3.5 \mathrm{E}-04$ & 2.3E-04 & La-140 & $6.0 \mathrm{E}-04$ & $3.9 \mathrm{E}-04$ \\
\hline Te-132 & $6.1 \mathrm{E}-04$ & $4.0 \mathrm{E}-04$ & La-141 & $1.2 \mathrm{E}-06$ & 7.7E-07 \\
\hline Te-133 & $2.3 \mathrm{E}-06$ & $1.6 \mathrm{E}-06$ & La-142 & $5.0 \mathrm{E}-05$ & 3.3E-05 \\
\hline $\mathrm{Te}-133 \mathrm{~m}$ & $2.8 \mathrm{E}-05$ & $1.8 \mathrm{E}-05$ & $\mathrm{Ce}-141$ & $1.1 \mathrm{E}-03$ & $7.3 \mathrm{E}-04$ \\
\hline Te-134 & $8.7 \mathrm{E}-06$ & $5.8 \mathrm{E}-06$ & $\mathrm{Ce}-143$ & $1.8 \mathrm{E}-04$ & $1.1 \mathrm{E}-04$ \\
\hline I-122 & $9.9 \mathrm{E}-09$ & $1.8 \mathrm{E}-08$ & Ce-144 & $1.8 \mathrm{E}-02$ & $1.2 \mathrm{E}-02$ \\
\hline I-123 & $2.1 \mathrm{E}-05$ & $4.6 \mathrm{E}-05$ & Pr-143 & 8.7E-04 & $5.7 \mathrm{E}-04$ \\
\hline $\mathrm{I}-125$ & $3.7 \mathrm{E}-02$ & $7.9 \mathrm{E}-02$. & Pr-144 & $6.7 \mathrm{E}-07$ & $4.7 \mathrm{E}-07$ \\
\hline
\end{tabular}


Table 4-13. 400 Area--CAP88-PC Unit Dose Factors by Effective Release Height ${ }^{\mathrm{a}}$ (mrem/Ci).

\begin{tabular}{|c|c|c|c|c|c|}
\hline \multirow{2}{*}{ Nuclide } & \multicolumn{2}{|c|}{$\begin{array}{c}\text { Effective } \\
\text { Release Height }\end{array}$} & \multirow{2}{*}{ Nuclide } & \multicolumn{2}{|c|}{$\begin{array}{c}\text { Effective } \\
\text { Release Height }\end{array}$} \\
\hline & $\mathrm{H}_{\mathrm{eff}}<40 \mathrm{~m}$ & $\mathrm{H}_{\text {eff }} \geq 40 \mathrm{~m}$ & & $\mathrm{H}_{\mathrm{eff}}<40 \mathrm{~m}$ & $\mathrm{H}_{\text {eff }} \geq 40 \mathrm{~m}$ \\
\hline Pr- $144 m$ & 4.4E-08 & $3.2 \mathrm{E}-08$ & Po-211 ${ }^{c}$ & 0.0 & 0.0 \\
\hline $\mathrm{Nd}-147$ & $8.5 \mathrm{E}-04$ & $5.6 \mathrm{E}-04$ & Po- $212^{c}$ & 0.0 & 0.0 \\
\hline $\mathrm{Pm}-147$ & $1.6 \mathrm{E}-03$ & $1.0 \mathrm{E}-03$ & Po- $213^{c}$ & 0.0 & 0.0 \\
\hline $\mathrm{Pm}-148$ & $1.3 \mathrm{E}-03$ & 8.3E-04 & Po-214 & 0.0 & 0.0 \\
\hline $\mathrm{Pm}-148 \mathrm{~m}$ & $1.1 \mathrm{E}-02$ & $7.5 \mathrm{E}-03$ & $\mathrm{Po}-215^{\mathrm{c}}$ & 0.0 & 0.0 \\
\hline Pm-149 & $1.6 \mathrm{E}-04$ & $1.0 \mathrm{E}-04$ & Po- $216^{\mathrm{c}}$ & 0.0 & 0.0 \\
\hline $\mathrm{Pm}-151$ & $4.3 \mathrm{E}-05$ & $2.8 \mathrm{E}-05$ & Po-218 & $1.7 \mathrm{E}-09$ & $1.3 \mathrm{E}-09$ \\
\hline Sm-147 & $2.5 E+00$ & $1.6 \mathrm{E}+\infty 0$ & At $-217^{\mathrm{c}}$ & 0.0 & 0.0 \\
\hline Sm-151 & $1.1 \mathrm{E}-03$ & $7.0 \mathrm{E}-04$ & $\operatorname{Rn}-219^{d}$ & $(\mathrm{~Pb}-211)$ & $(\mathrm{Pb}-211)$ \\
\hline Sm-153 & 1.1E-04 & 7.4E-05 & $\mathrm{Bn}-220^{\mathrm{d}}$ & $(\mathrm{Pb}-212)$ & $(\mathrm{Pb}-212)$ \\
\hline Eu-152 & $3.6 \mathrm{E}-01$ & $2.4 \mathrm{E}-01$ & $\mathrm{Rn}-222$ & $9.5 \mathrm{E}-05$ & $5.4 \mathrm{E}-05$ \\
\hline $\mathrm{Eu}-152 \mathrm{~m}$ & $1.6 \mathrm{E}-05$ & $1.0 \mathrm{E}-05$ & Fr-221 & $1.3 \mathrm{E}-06$ & $9.8 \mathrm{E}-07$ \\
\hline Eu-154 & $2.9 \mathrm{E}-01$ & $1.9 \mathrm{E}-01$ & Fr-223 & $2.4 \mathrm{E}-05$ & $1.6 \mathrm{E}-05$ \\
\hline Eu-155 & $1.2 \mathrm{E}-02$ & $7.7 \mathrm{E}-03$ & $\mathrm{Ra}-223$ & $3.5 \mathrm{E}-01$ & 2.3E-01 \\
\hline Eu-156 & $3.6 \mathrm{E}-03$ & $2.4 \mathrm{E}-03$ & Ra-224 & $1.3 \mathrm{E}-01$ & 8.1E-02 \\
\hline$G d-152^{b}$ & (Pu-239) & (Pu-239) & $\mathrm{Ra}-225$ & $2.2 \mathrm{E}-01$ & $1.4 \mathrm{E}-01$ \\
\hline $\mathrm{Tb}-160$ & $9.4 \mathrm{E}-03$ & $6.2 \mathrm{E}-03$ & $\mathrm{Ra}-226$ & $6.7 \mathrm{E}-01$ & $4.4 \mathrm{E}-01$ \\
\hline Ho-166 & $1.4 \mathrm{E}-04$ & $8.6 \mathrm{E}-05$ & $\mathrm{Ra}-228$ & $2.8 \mathrm{E}-01$ & $1.8 \mathrm{E}-01$ \\
\hline Ho-166m & $1.2 \mathrm{E}+00$ & 7.7E-01 & Ac- 225 & 2.1E-01 & $1.3 \mathrm{E}-01$ \\
\hline Hf-181 & $3.6 \mathrm{E}-03$ & $2.4 \mathrm{E}-03$ & Ac-227 & $2.2 \mathrm{E}+01$ & $1.4 \mathrm{E}+01$ \\
\hline W-181 & $6.6 \mathrm{E}-04$ & $4.4 \mathrm{E}-04$ & Ac-228 & $2.5 \mathrm{E}-03$ & $1.6 \mathrm{E}-03$ \\
\hline W-185 & $8.8 \mathrm{E}-04$ & $5.8 \mathrm{E}-04$ & Th-227 & $3.8 \mathrm{E}-01$ & $2.4 \mathrm{E}-01$ \\
\hline W-187 & 7.7E-05 & $5.0 \mathrm{E}-05$ & Th-228 & $8.2 \mathrm{E}+00$ & $5.2 \mathrm{E}+00$ \\
\hline Re-187 & $2.5 \mathrm{E}-05$ & $1.7 \mathrm{E}-05$ & Th-229 & $2.3 E+01$ & $1.5 \mathrm{E}+01$ \\
\hline Ir -192 & $8.0 \mathrm{E}-03$ & $5.3 \mathrm{E}-03$ & Th-230 & $8.2 \mathrm{E}+00$ & $5.2 \mathrm{E}+00$ \\
\hline $\mathrm{Hg}-203$ & $5.0 \mathrm{E}-03$ & $3.3 \mathrm{E}-03$ & Th-231 & $3.6 \mathrm{E}-05$ & $2.3 \mathrm{E}-05$ \\
\hline Tl-207 & $2.8 \mathrm{E}-09$ & 2.1E-09 & Th-232 & $1.2 \mathrm{E}+01$ & $7.4 \mathrm{E}+00$ \\
\hline Tl-208 & $7.4 \mathrm{E}-08$ & $5.6 \mathrm{E}-08$ & Th-234 & $3.5 \mathrm{E}-03$ & $2.3 \mathrm{E}-03$ \\
\hline T1-209 & 4.2E-09 & $3.2 \mathrm{E}-09$ & $\mathrm{~Pa}-231$ & $1.7 \mathrm{E}+01$ & $1.1 \mathrm{E}+01$ \\
\hline $\mathrm{Pb}-209$ & $2.7 \mathrm{E}-06$ & $1.7 \mathrm{E}-06$ & $\mathrm{~Pa}-233$ & $1.5 \mathrm{E}-03$ & $1.0 \mathrm{E}-03$ \\
\hline $\mathrm{Pb}-210$ & $2.1 \mathrm{E}+00$ & $1.4 \mathrm{E}+00$ & $\mathrm{~Pa}-234$ & $1.1 \mathrm{E}-04$ & $7.2 \mathrm{E}-05$ \\
\hline $\mathrm{Pb}-211$ & $1.3 \mathrm{E}-04$ & $8.7 \mathrm{E}-05$ & $\mathrm{~Pa}-234 \mathrm{~m}$ & $3.0 \mathrm{E}-14$ & $2.2 \mathrm{E}-14$ \\
\hline $\mathrm{Pb}-212$ & $5.1 \mathrm{E}-03$ & $3.3 \mathrm{E}-03$ & U-232 & $1.6 \mathrm{E}+01$ & $1.0 \mathrm{E}+01$ \\
\hline $\mathrm{Pb}-214$ & $1.2 \mathrm{E}-05$ & $8.0 \mathrm{E}-06$ & U-233 & $4.6 \mathrm{E}+00$ & $2.9 \mathrm{E}+00$ \\
\hline $\mathrm{Bi}-210$ & $6.6 \mathrm{E}-03$ & 4.2E-03 & U-234 & $4.5 E+00$ & $2.9 \mathrm{E}+\infty 0$ \\
\hline $\mathrm{Bi}-211$ & $2.8 \mathrm{E}-09$ & $2.1 \mathrm{E}-09$ & U-235 & $4.3 E+00$ & $2.8 \mathrm{E}+00$ \\
\hline Bi-212 & $6.3 \mathrm{E}-04$ & $4.1 \mathrm{E}-04$ & $\mathrm{U}-236$ & $4.3 E+00$ & $2.7 \mathrm{E}+00$ \\
\hline $\mathrm{Bi}-213$ & 2.1E-05 & $1.4 \mathrm{E}-05$ & U-237 & $4.5 \mathrm{E}-04$ & $3.0 \mathrm{E}-04$ \\
\hline $\mathrm{Bi}-214$ & $1.1 \mathrm{E}-05$ & $7.6 \mathrm{E}-06$ & U-238 & $4.1 E+\infty 0$ & $2.6 \mathrm{E}+00$ \\
\hline Po-210 & $7.7 \mathrm{E}-01$ & $5.0 \mathrm{E}-01$ & $U-240$ & $7.9 \mathrm{E}-05$ & $5.0 \mathrm{E}-05$ \\
\hline
\end{tabular}


Table 4-13. 400 Area--CAP88-PC Unit Dose Factors by Effective Release Height ${ }^{a}$ (mrem/Ci).

\begin{tabular}{|c|c|c|c|c|c|}
\hline \multirow{2}{*}{ Nuclide } & \multicolumn{2}{|c|}{$\begin{array}{c}\text { Effective } \\
\text { Release Height }\end{array}$} & \multirow{2}{*}{ Nuclide } & \multicolumn{2}{|c|}{$\begin{array}{c}\text { Effective } \\
\text { Release Height }\end{array}$} \\
\hline & $\mathrm{H}_{\text {eff }}<40 \mathrm{~m}$ & $\mathrm{H}_{\text {eff }} \geq 40 \mathrm{~m}$ & & $\mathrm{H}_{\text {eff }}<40 \mathrm{~m}$ & $\mathrm{H}_{\text {eff }} \geq 40 \mathrm{~m}$ \\
\hline Np-237 & $1.7 \mathrm{E}+01$ & $1.1 \mathrm{E}+01$ & & & \\
\hline $\mathrm{Np}-238$ & $1.2 \mathrm{E}-03$ & 7.5E-04 & & & \\
\hline $\mathrm{Np}-239$ & $1.8 \mathrm{E}-04$ & $1.2 \mathrm{E}-04$ & & & \\
\hline $\mathrm{Np}-240$ & $1.8 \mathrm{E}-05$ & $1.2 \mathrm{E}-05$ & & & \\
\hline $\mathrm{Np}-240 \mathrm{~m}$ & $2.7 \mathrm{E}-07$ & $2.0 \mathrm{E}-07$ & & & \\
\hline$P u-236$ & $3.0 E+00$ & $1.9 E+\infty 0$ & & & \\
\hline Pu-238 & $1.1 \mathrm{E}+01$ & $7.1 E+00$ & & & \\
\hline Pu-239 & $1.2 \mathrm{E}+01$ & $7.7 \mathrm{E}+00$ & & & \\
\hline $\mathrm{Pu}-240$ & $1.2 \mathrm{E}+01$ & $7.7 \mathrm{E}+00$ & & & \\
\hline Pu-241 & $1.9 \mathrm{E}-01$ & $1.2 \mathrm{E}-01$ & & & \\
\hline Pu-242 & $1.2 \mathrm{E}+01$ & $7.3 E+00$ & & & \\
\hline $\mathrm{Pu}-243$ & 7.4E-06 & 4.7E-06 & & & \\
\hline $\mathrm{Pu}-244$ & 1.1E+01 & $7.3 E+\infty$ & & & \\
\hline Am-241 & $1.9 \mathrm{E}+01$ & $1.2 \mathrm{E}+01$ & & & \\
\hline Am-242 & $1.9 \mathrm{E}-03$ & $1.2 \mathrm{E}-03$ & & & \\
\hline $\mathrm{Am}-242 \mathrm{~m}$ & $1.8 \mathrm{E}+01$ & $1.1 \mathrm{E}+01$ & & & \\
\hline Am-243 & $1.9 \mathrm{E}+01$ & $1.2 \mathrm{E}+01$ & & & \\
\hline $\mathrm{Cm}-242$ & $6.0 \mathrm{E}-01$ & $3.8 \mathrm{E}-01$ & & & . \\
\hline $\mathrm{Cm}-243$ & $1.3 \mathrm{E}+01$ & $7.9 \mathrm{E}+\infty 0$ & & & \\
\hline $\mathrm{Cm}-244$ & $9.8 E+00$ & $6.2 \mathrm{E}+00$ & & & \\
\hline $\mathrm{Cm}-245$ & $1.9 \mathrm{E}+01$ & $1.2 \mathrm{E}+01$ & & & . \\
\hline $\mathrm{Cm}-246$ & $1.9 \mathrm{E}+01$ & $1.2 \mathrm{E}+01$ & & & \\
\hline $\mathrm{Cm}-247$ & $1.8 \mathrm{E}+01$ & $1.1 E+01$ & & & \\
\hline $\mathrm{Cm}-248$ & $7.0 \mathrm{E}+01$ & $4.4 \mathrm{E}+01$ & & & \\
\hline Cf-252 & $5.2 \mathrm{E}+00$ & $3.3 E+\infty 0$ & & & \\
\hline
\end{tabular}

The effective release height, $x$, is in meters.

b CAP88-PC Ver. 1.0 does not calculate doses for Be-10, Se-79, Cd-113, Cd-113m, and Gd-152. Recommend substituting the unit dose factors of the nuclides in parentheses.

c Extremely short lived nuclide, resulting in an offsite dose of zero.

d Short lived radon nuclides only provide the mechanism to circumvent the emission control device. The unit dose factors for the recommended progeny should be used instead. However, 1 ci of Rn does not equal $1 \mathrm{ci}$ of $\mathrm{Pb}$. Multiply the activity of Rn-219 by 0.0018 to get the activity of $\mathrm{Pb}-211$. Multiply the activity of $\mathrm{Rn}-220$ by 0.0014 to get the activity of $\mathrm{Pb}-212$. 
HNF-3602, Vol. 1

This page intentionally left blank. 
HNF-3602, Vol. 1

\subsection{PEER REVIEW DOCUMENTATION}

This section provides the peer review documentation as required by the Hanford Environmental Dose Overview Panel's manual titled Recommended Environmental Dose Calculation Methods and Hanford-Specific Parameters (PNL-3777). 


\section{PEER REVIEW CHECKIIST}

Document Reviewed:

Scope of Review:
Calculating Potential-to-Emit Releases and Doses for FEMPs and NOCs

Use of CAP88-PC and GENII to Generate Unit Dose Factors

Yes No NA

IV [] [ ] Necessary assumptions explicitly stated and supported.

[V] [ ] [ ] Computer codes and data files identified in document.

[M] [ ] [ ] Data used in calculations explicitly stated in document.

[ ] [ ] $M$ ] Data checked for consistency with original source information as applicable.

[ ] [ ] [/ Mathematical derivations checked including dimensional consistency of results.

$M$ [ ] [ ] Models appropriate and used within range of validity or use outside range of established validity justified.

$\checkmark[$ [ [ ] Hand calculations (including spreadsheets) checked for errors.

L] [ ] [ ] Software input correct and consistent with descriptions in the document.

[ $]$ [ ] [ ] software output consistent with input and with results reported in the document.

[ ] [ ] $\$ Safety margins consistent with good engineering practices.

4 [ ] Document approved by: Kathleen Rhoads

Signature:



Date: $4 / 15 / 99$ 
HNF-3602, Vol. 1

HEDOP REVIEW CHECKLIST

for Rad and Non-Rad Environmental Releases

Document Reviewed:

Calculating Potential-to-Emit Releases and Doses for FEMPs and. NOCs

Scope of Review:

Use of CAP88-PC and GENII to Generate Unit Dose Factors

YES NO* N/A

[ ] [ ] [ ] 1. A detailed technical review and approval of the envirronmental transport and dose calculation portion of the analysis has been performed and documented.

[V [ ] [ ] 2. Detailed technical review(s) and approval(s) of scenario and release determinations have been performed and documented.

[V] [ ] [ ] 3. HEDOP-approved code (s) were used.

M] [ ] [ ] 4. Receptor locations were selected according to HEDOP recommendations.

V] [ ] [ ] 5. AIl applicable environmental pathways and code options were included and are appropriate for the calculations.

V] [ ] [ ] 6. Hanford site data were used:

[ ] [ ] M] 7. Model adjustments external to the computer program were justified and performed correctly.

4 [ ] [ ] 8. The analysis is consistent with HEDOP recommendations.

[ ] $\sqrt{ }]$ 9. Supporting notes, calculations, comments, comment resolutions, or other information is attached.

(Use the "Page $I$ of $X "$ page numbering format and sign and date each added page.)

4 [ ] 10. Approval is granted on behalf of the Hanford Environmental Dose Overview Panel.

* All "NO" responses must be explained and use of nonstandard methods justified.

Reviewer Name: Kathleen Rhoads

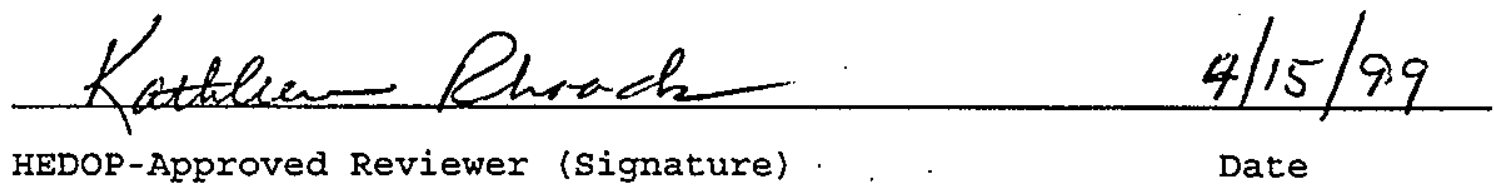

COMMENTS (add additional signed and dated pages if necessary): 
HNF-3602, Vol. 1

This page intentionally left blank. 


\subsection{REFERENCES}

ASME/ANSI N509, Testing of Nuclear Air Treatment Systems, American Society of Mechanical Engineers/American National Standards Institute.

ASTM E 29, Standard Practice for Using Significant Digits in Test Data to Determine Conformance with Specifications, American Society for Testing and Materials, Annual Book of Standards, Volume 14.02, Designation E 29.

Barnett, J. M., and W. E. Davis, 1996, "Six Methods to Assess Potential Radioactive Air Emissions from a Stack," Health Physics, Vol. 71, No. 5, November, pp 773-778.

DOE Order 5400.1, General Environmental Protection Program.

DOE Order 5400.5, Radiation Protection of the Public and the Environment.

DOE/RL-96-75, Radioactive Air Emissions Notice of Construction Portable/Temporary Radioactive Air Emission Units, U.S. Department of Energy, Richland Operations Office, Richland, Washington.

DOE/TIC-11026, 1981, Radioactive Decay Data Tables, Oak Ridge National Laboratory.

ERDA 76-21, Nuclear Air Cleaning Handbook, Oak Ridge National Laboratory, Oak Ridge, Tennessee.

Parks, B. S., 1992, CAP88-PC Version 2.0 User's Guide, U.S. Department of Energy, Germantown, Maryland.

PNL-3777, Recommended Environmental dose Calculation Methods and Hanford-Specific Parameters, Pacific Northwest National Laboratory, Richland, Washington.

PNL-6584, GENIL-the Hanford Environmental Radiation Dosimetry Software System, Vols. 1-3, Pacific Northwest Laboratory, Richland, Washington.

PNNL-11794, Hanford Site Climatological Data Summary 1997, with Historical Data, March 1998, Pacific Northwest National Laboratory.

Sutter, S. L., J. W. Johnston, J. Mishima, 1981, Aerosols Generated by Free Fall Spills of Powders and Solutions in Static Air, NUREG/CR-2138 PNL-3786, U.S. Nuclear Regulatory Commission.

WHC-EP-0498, Unit Dose Calculation Methods and Summary of Facility Effluent Monitoring Plan Determinations, November 1991, Westinghouse Hanford Company, Richland, Washington.

WHC-SD-ER-TP-004, Measurement of Gamma Activity from HEPA Filters, Rev. 1, April, 1993, Westinghouse Hanford Company, Richland, Washington. 
HNF-3602, Vol. 1

This page intentionally left blank. 
HNF-3602, Vol. 1

\section{DISTRIBUTION}

U.S. Department of Energy, Richland Operations Office

MSIN

H. M. Rodriguez

A5-15

J. B. Hall

A. V. Ingle

A5-15

A5-15

Pacific Northwest National Laboratory

Hanford Technical Library

$\mathrm{K} 1-11$

Fluor Daniel Hanford, Inc.

W. D. Adair

H6-21

J. A. Bates

H6-23

J. K. Perry

H6-25

D. G. Ranade

H6-23

Lockheed Martin Hanford Corporation

D. J. Carrell

R1-51

D. E. Clark

R1-51

G. M. Crummel

R1-51

D. L. Dyekman

S7-01

P. C. Miller

R1-51

Waste Management Federal Services of Hanford, Inc.

R.B. Barmettlor

T4-04

B. M. Barnes

T4-04

H. C. Boynton

T4-52

B. L. Curn

H6-36

W. E. Davis

H6-36

L. P. Diediker (10)

H6-36

T. P. Frazier

D. W. Fritz

C. J. Grando

E. M. Greager

R. D. Haggard

H6-25

H6-36

H6-36

H6-36

J. S. Hill

S6-71

N. A. Homan

H6-25

. Kamber

H6-25

R. E Johnson

H6-25

J. J. Luke

H6-25

R. W. Szelmeczka

H6-25

W. R. Thackaberry

L6-05

T4-52

D. D. Volkman

T3-05 
B\&W Hanford Co.

J. M. Barnett

J. E. Bramson

T5-54

G. J. Lebaron

T3-05

K. S. McDaniel

S6-70

D. E. Rasmussen

Duke Engineering and Services Hanford

R.G. Gant

D.J. Watson

Lockheed Martin Services, Inc.

Central Files

Bechtel Hanford, Inc.

E. T. Coenenberg

R. L. Collins

$\mathrm{H} 0-16$

K. E. Cook

H9-02

R. J. Landon

$\mathrm{HO}-02$

J. D. Ludowise

H9-03

J. G. Woolard H0-17

Fluor Daniel Northwest, Inc.

D. A. Himes

B4-47

R. L. Newell

B4-68

R. J. Puigh II

B4-43

P. D. Rittman

R3-23

J. C. Van Keuren

B4-47 